\title{
The ELMO Bumpy Torus Reactor (EBTR) Reference Design
}

\author{
D. G. McAlees \\ - N. A. Uckan \\ E. S. Bettis \\ C. L. Hedrick \\ E. F. Jaeger \\ D. B. Nelson \\ L. W. Owen \\ J. F. Roberts \\ R. T. Santoro
}
D. A. Spong
H. L. Watts
H. T. Yeh
L. M. Lidsky
D. A. Ehst
J. S. Herring
D. L. Kaplan
A. Pant
R. E. Potok

\section{OAK RIDGE NATIONAL LABORATORY}




\section{DISCLAIMER}

This report was prepared as an account of work sponsored by an agency of the United States Government. Neither the United States Government nor any agency Thereof, nor any of their employees, makes any warranty, express or implied, or assumes any legal liability or responsibility for the accuracy, completeness, or usefulness of any information, apparatus, product, or process disclosed, or represents that its use would not infringe privately owned rights. Reference herein to any specific commercial product, process, or service by trade name, trademark, manufacturer, or otherwise does not necessarily constitute or imply its endorsement, recommendation, or favoring by the United States Government or any agency thereof. The views and opinions of authors expressed herein do not necessarily state or reflect those of the United States Government or any agency thereof. 


\section{DISCLAIMER}

Portions of this document may be illegible in electronic image products. Images are produced from the best available original document. 
Printed in the United States of America. Available from Natinnal Technical Information Service

U.S. Department of Commerce

5285 Port Royal Road, Springfield, Virginia 22161

Price: Printed Copy $\$ 6.00$; Microfiche $\$ 2.25$

This report was prepared as an account of work sponsored by the United States Government. Neither the United States nor the Energy Research and Development Administration/United States Nuclear Regulatory Commission, nor any of their employees, nor any of their contractors, subcontractors, or their employees, makes any warranty, express or implied, or assumes any legal liability or responsibility for the accuracy, completeness or usefulness of any information, apparatus, product or process disclosed, or represents that its use wuuld not infringe privately owned rights. 
Contract No. W-7405-eng-26

\author{
FUSION ENERGY DIVISION \\ ENGINEERING DIVISION \\ COMPUTER SCIENCES DIVISION \\ NEUTRON PHYSICS DIVISION
}

THE ELMO BUMPY TORUS REACTOR (EBTR) REFERENCE DESIGN
D. G. McAlees
N. A. Uckan
L. W. Owen
E. S. Bettis
J. F. Roberts
C. L. Hedrick
E. F. Jaeger
R. T. Santoro
D. B. Nelson
D. A. Spong
H. L. Watts
H. T. Yeh

Oak Ridge National Laboratory, Oak Ridge, Tennessee

and
L. M. Lidsky
D. A. Ehst
D. L. Kaplan
J. S. Herring
A. Pant
R. E. Potok

Massachusetts Institute of Technology, Cambridge, Massachusetts

02139

Date Published: November 1976

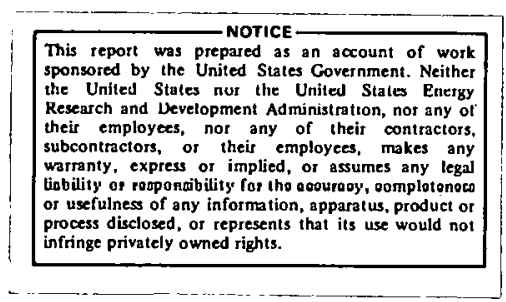

OAK RIDGE NATIONAL LABORATORY

Oak Ridge, Tennessee 37830

operated by

UNION CARB IDE CORPORATION

for the

ENERGY RESEARCH AND DEVELOPMENT ADMINISTRATION 
THIS PAGE

\section{WAS INTENTIONALLY LEFT BLANK}


THE ELMO BUMPY TORUS REACTOR (EBTR) REFERENCE DESIGN*

D. G. McAlees ${ }^{1,2}$, N. A. Uckan ${ }^{2}$, E. S. Bettis ${ }^{3}$, C. L. Hedrick ${ }^{2}$, E. F. Jaeger ${ }^{2}$, D. B. Nelson ${ }^{2}$, L. W. Owen ${ }^{4}$, J. F. Roberts ${ }^{4}$, R. T. Santoro ${ }^{5}$, D. A. Spong ${ }^{2}$, H. L. Watts ${ }^{3}$, H. T. Yeh ${ }^{2}$

Oak Ridge National Laboratory, Oak Ridge, Tennessee 37830

and

L. M. Lidsky, D. A. Ehst ${ }^{6}$, J. S. Herring, D. L. Kaplan, A. Pant, R. E. Potok

Massachusetts Institute of Technology, Cambridge, Massachusetts 02139

ABSTRACT

The goal of the ELMO Bumpy Torus Reactor (EBTR) study is the evaluation of the EBT confinement concept as the basis for development of a commercial fusion power reactor. A multidisciplinary, selfconsistent treatment of EBT reactor scaling and design has been completed and a reference design (EBTR-48) has been developed. This design, based on a realistic plasma model and relatively conservative engineering parameters (i.e., $1 \mathrm{MW} / \mathrm{m}^{\text {? }}$ neutron wall loading and a $7.3 \mathrm{~T}$ maximum toroidal field), is a steady state, ignited-mode system with high plasma power density and aspect ratio. The total thermal power of EBTR-48, exclusive of blanket multiplication, is $4000 \mathrm{MW}$; the design is based on a standard module and the design power level for a particular plant is determined by the number of modules used. Several design variants have been investigated in detail to illustrate the effect of nearterm and advanced technologies and to illustrate the design freedom offered by devices with low field and high aspect ratio. The high aspect ratio simplifies many aspects of the design, most notably those associated with remote maintenance, accessibility, and repair. It appears that a commercially successful EBTR could be constructed with only slight advances in existing technology, if the present understanding of the physics can be extrapolated to the reactor regime and does not differ markedly from the model developed for this study.

*Research sponsored by the Energy Research and Development Administration under contract with Union Carbide Corporation.

${ }^{1}$ Exxon Nuclear Co., Inc.

${ }^{2}$ Fusion Energy Division

${ }^{3}$ Engineering Division

${ }^{4}$ Computer Sciences Division

${ }^{5}$ Neutron Physics Division

${ }^{6}$ Present address, Argonne National Laboratory 
THIS PAGE

WAS INTENTIONALLY

LEFT BLANK 


\section{ACKNOWLEDGMENT}

There has been considerable interest in the EBT reactor study. The contributions of many people are reflected in this summary. This broad input is vital to a study of this type. Unfortunately, it is not possible to credit everyone explicitly. Specifically, the support of and discussions with R. A. Dandl and R. A. Dory have been especially helpful in this work. 
THIS PAGE

WAS INTENTIONALLY

LEFT BLANK 


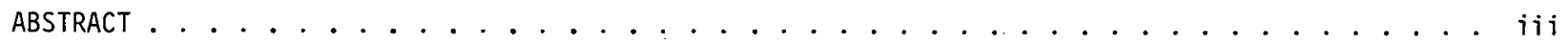

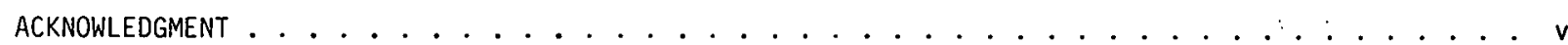

EXECUTIVE SUMMARY

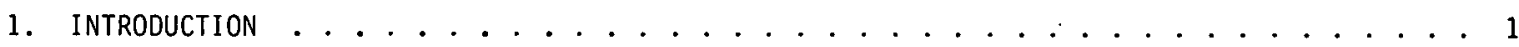

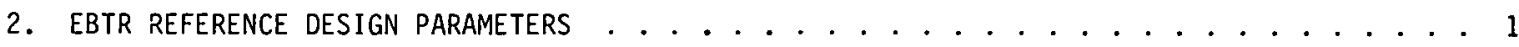

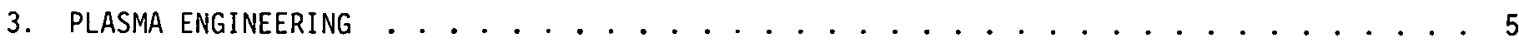

3.1 System Characteristics . . . . . . . . . . . . . . . 6

3.2 system Economics . . . . . . . . . . . . . . . . . . 7

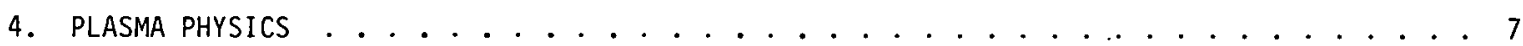

4.1 Equilibrium and Drift Surfaces . . . . . . . . . . . . . 7

4.2 Macroscopic Stability . . . . . . . . . . . . . . . 8

4.3 Scaling, Transport, and Modeling .................. 10

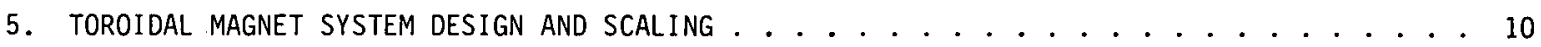

5.1 Magnet Scaling . . . . . . . . . . . . . . . . . 10

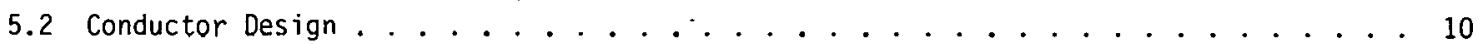

5.3 Magnet Design . . . . . . . . . . . . . . . . 10

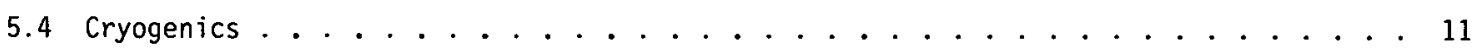

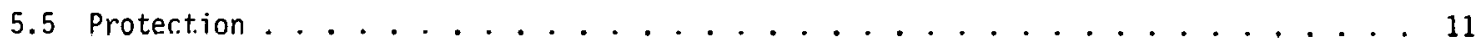

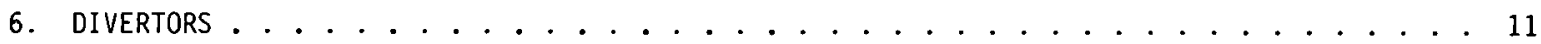

6.1 Divertor Requirements . . . . . . . . . . . . . . . 12

6.2 Divertor Field Design . . . . . . . . . . . . . . . . 12

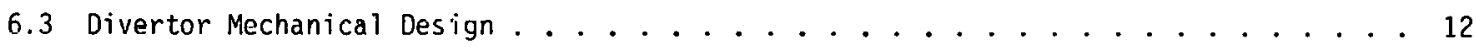

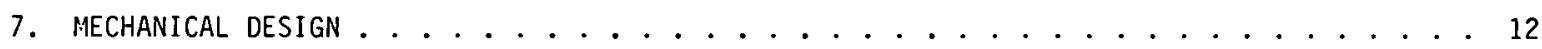

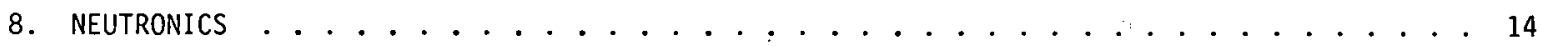

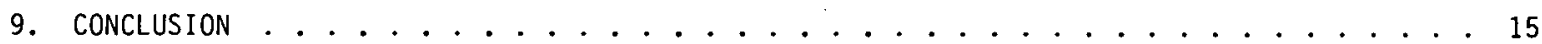

APPENDIXES

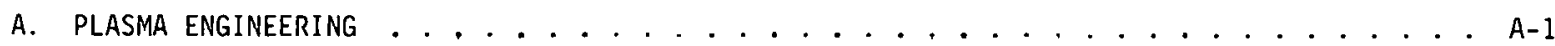

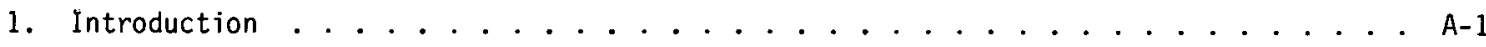

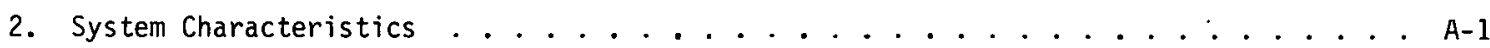

2.1 Neutron Wall Loading : .................... . . . . . . .

2.2 Plasma Thermal Power Density . . . . . . . . . . . . A-3

2.3 Plasma Parameters . . . . . . . . . . . . . . A-4

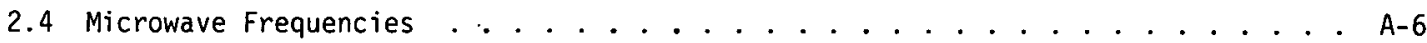

2.5 Mirror Ratio and Magnet Spacing .................... A-8

2.6 Reference Parameters ................... . . . . . . . . . .

3. System Economics . . . . . . . . . . . . . . . . A A-9

3.1 Power Balance and Economic Constraints . . . . . . . . . . A A-10

3.1.1 Recirculating power fraction and conversion efficiency ........ A-10

3.1 .2 Reactor cost dependence on $Q^{*} \ldots \ldots \ldots \ldots$. . . . . . . . . .

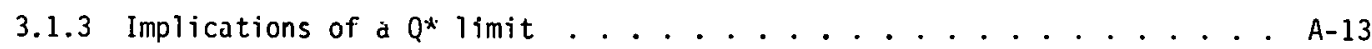

3.2 EBT Reactor cost Scaling . . . . . . . . . . . . . A-15

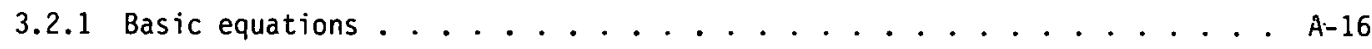

3.2.2 Cost comparisons and options available ............. A-16 
B. PLASMA PHYSICS

1. Introduction ............................... B-1

2. Equilibrium and Drift Surfaces ....................... . B-1

3. Macroscopic Stability ......................... . B-10

3.1 Stability of the Hot Electron Annuli . . . . . . . . . . . . . . B-10

3.2 Stability and Beta Limitations of the Bulk Plasma . . . . . . . . . . . . B-11

C. CYCLOTRON RADIATION

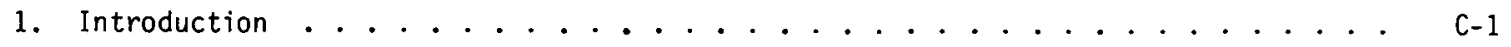

2. Formulation of Radiation Losses ......................... . C-1

D. PLASMA SIMUIIATION AND MODELING

1. Introduction .............................. D 1

2. Physics Mòdél . . . . . . . . . . . . . . . . . . . . . . . D-1

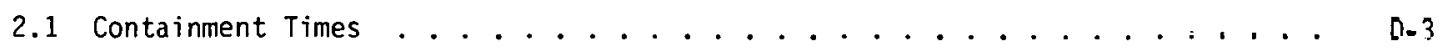

2.2 Radiation Losses. . . . . . . . . . . . . . . . . . D-3

2.3 Alpha Energy Deposition ..................... . . . 0 . . 4

2.4 Neutral Beam Injection Heating.................... D D

2.5 Further Comments on the Model ................... D-4

3. EBT start-Up ............................... . . . . . . . . . . .

E. TOROIDAL MAGNET SYSTEM DESIGN

1. Introduction . . . . . . . . . . . . . . . . . . . . . . . . E-1

2. Magnet Scaling Laws........................... . E-1

3. Conductor Design ........................... . E-6

3.1 Deslyn Crileria . . . . . . . . . . . . . . . . . . . E-6

3.2 D̃escription ........................ . . E-6

4. Magnet Design . . . . . . . . . . . . . . . . . . . . E E-8

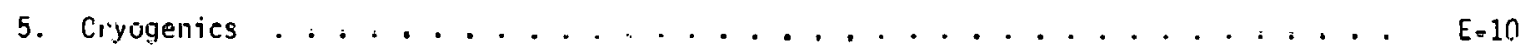

6. Protection Uesigñ ............................. . E-12

6.1 Design Criteria ....................... E E-12

6.2 Description ...................... E-12

7. EBTR-48 Alternate Magnet Design . . . . . . . . . . . . . . . . . E-14

7.1 Conductor Design ...................... . . E-14

7.2 Force Calculations......................... . . [ . . . . . . . E

7.3 Forced Shutdown ....................... . . E-16

7.4 Dewars for Alternate Magnet Uesign . . . . . . . . . . . . . . F-16

8. Summary of Magnet Design . . . . . . . . . . . . . . . . . . . E-18

F. DIVERTORS

1. Introduction .............................. . . F-1

2. Divertor Requirements ........................ . . F-1

3. Divertor Field Design ........................... . . F-1

4. Divertor Mechanical Design ......................... . . F-3 
G. MECHANICAL DESIGN

1. Introduction . . . . . . . . . . . . . . . . . . . . G-1

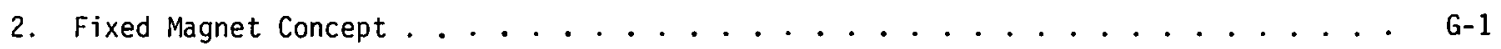

3. Blanket and Heat Removal....................... . . . G-6

4. Reactor Maintenance ... . . . . . . . . . . . . . . . . . . . G-8

5. Heat Losses . . . . . . . . . . . . . . . . . . . . . . G G

6. Movable Magnet Concept .......................... G-10

H. NEUTRONICS CALCULATIONS

1. Introduction . . . . . . . . . . . . . . . . . . . . . . . . H-1

2. Preliminary Neutronics Analysis ....................... H-l

3. Analysis of Alternate Blanket-Shield Designs . . . . . . . . . . . . . . H-6

4. Future Neutronics Calculations .. . . . . . . . . . . . . . . . . . H-8 
EXECUTIVE SUMMARY 


\section{INTRODUCTION}

Fusion reactor system studies to date dealt primarily with the tokamak confinement concept. These studies showed that physics criteria lead to difficult engineering problems. Specifically, a low beta plasma confined in a low aspect ratio torus yields a lower power density, nonuniform structural loadings because of the strong curvature, and accessibility problems. Tokamaks require pulsed magnetic fields which complicate the structural design. Although recent theoretical advances in tokamak plasma physics suggest that some of the criteria may be relaxed, ${ }^{1,2}$ it is prudent to examine promising alternate sys tems.

The design of a complex commercial power reactor requires the self-consistent analysis of interacting systems. The design presented here was carried out by a team of engineers and physicists from the Oak Ridge National Laboratory (ORNL) and the Massachusetts Institute of Technology (MIT).

A summary of the first reactor study based on the EBT confinement ${ }^{3}$ concept is presented here. It is assumed that the physics will extrapolate favorably to the reactor regime; if so, the EBT reactor would operate at high beta and high power density, and in steady state. No pulsed magnetic fields are required. EBT is a high aspect ratio device, so the accessibility problems are virtually eliminated, remote maintenance difficulties are eased, and relatively uniform structural loadings, due to weak curvature, alleviate engineering design problems. The EBT experiment has demonstrated stable plasma operation and obtained results which further encourage the reactor study. ${ }^{4}$

The reference design has been made consistent with the requirements of plasma engineering, plasma physics, magnetics, neutronics, and design engineering. The most important considerations in each of these areas are given in this summary report; the detailed work underlying the conclusions is presented in the appendixes. There are no unique solutions to such design problems - the "optimum design" is strongly dependent on the weight given to each of the constraints. For EBT-based systems, a small number of design choices is sufficient to indicate the major aspects of the final design: the choice of wall loading, mirror ratio, and limiting plasma pressure determines thesize of a standard module of the system and, given total plant output, the number of modules in the system. Similarly, constraints of power circulation efficiency require a commercial reactor be operated in the steady state, ignited mode.

The reference design presented is based on scaling arguments. One of the most important constraints was the imposed $\sim 1 \mathrm{MW} / \mathrm{m}^{2}$ neutron wall loading limit. Studies in progress indicate that the optimum wall loading in a commercial plant could be higher: in the range of $2-4 \mathrm{MW} / \mathrm{m}^{2}$. A smaller reference design is under development which would operate near the optimum wall loading. The present reference design is cuilservative in an absolute sense and relative to other fusion reactor design studies. Nonetheless, the reactor outlined here as EBTR-48 is attractive as a commercial plant and could be built with only minor extension of present technology.

\section{EBTR REFERENCE DESIGN PARAMETERS}

An FRTR reference design has been developed. Preliminary power balance calculations suggest that the toroidal plasma should be heated to ignition conditions using neutral beam injection and that the stabilizing relativislic electron rings should be sustained by microwaves. Figure 1 is a plan view of the system. Table 1 shows the fundamental parameters for the system. The power output and neutron wall loading in the EBTR have been taken to be similar to the values assumed in low beta tokamak system studies in order to facilitate comparisons. 


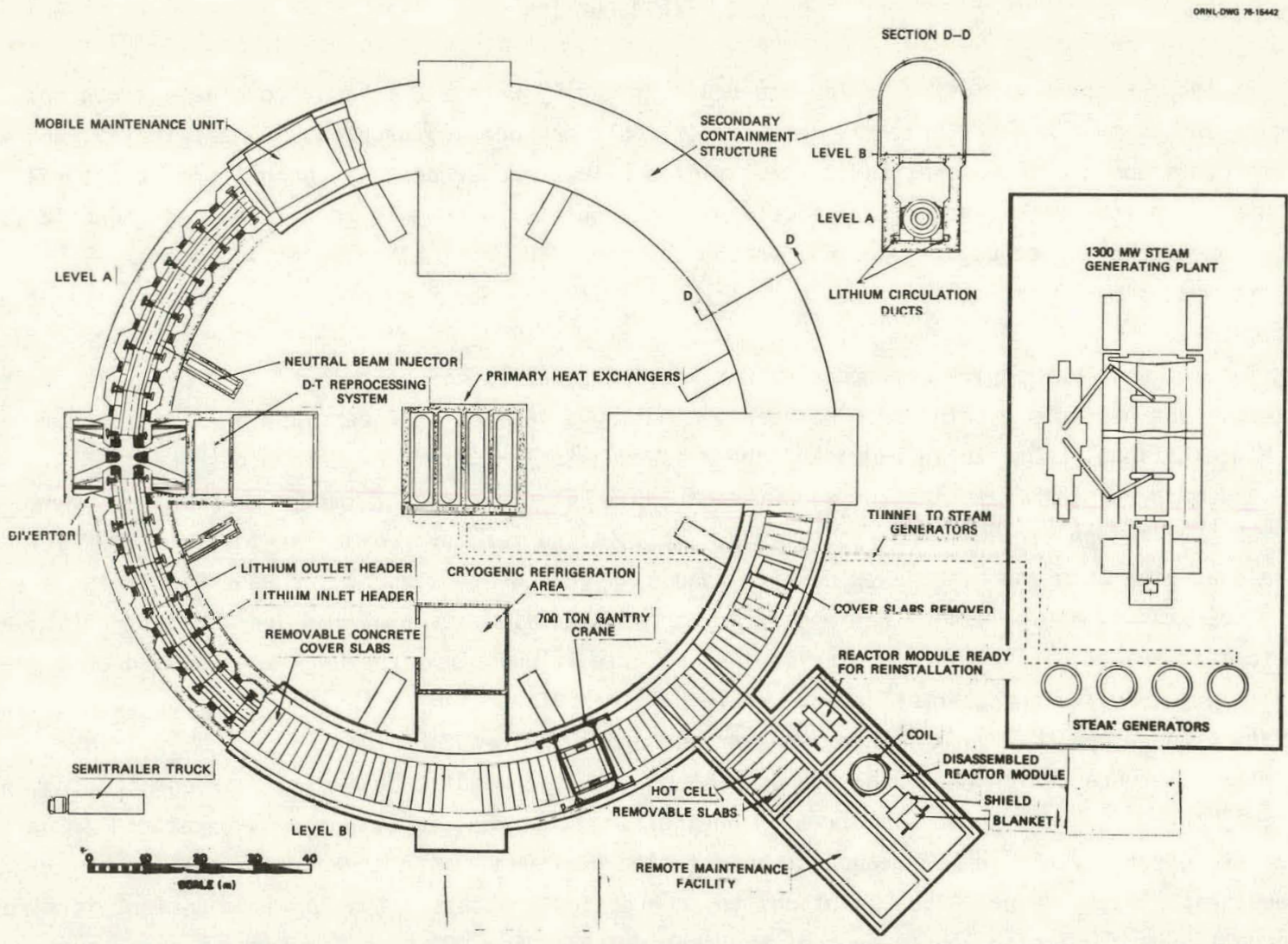

rig. 1. EBTR plan view

The reactor is fueled by deuterium and tritium and operates in the ignited mode. It produces $4000 \mathrm{MW}(\mathrm{th})$, exclusive of blanket multiplication. This is consistent with a source neutron wall lnading of $1.1 \mathrm{MW} / \mathrm{m}^{2}$ and a fusion power density of $3.4 \mathrm{MW} / \mathrm{m}^{3}$. The confining magnetic field at the midplane is $2.5 \mathrm{~T}$ which gives a beta of 0.25 . The magnetic field is produced by forty-eight 6 -m-bore superconducting coils. The mirror raliu is 1.8 so that the ficld strength in d llignet throat is $4.5 \mathrm{~T}$. The major radius of the device is $60 \mathrm{~m}$, and the plasma radius is $1 \mathrm{~m}$, so that the plasma aspect ratio is 60 . The self-consistent relationships between the geometrical and power production parameters are shown in Fig. 2, and discussed in detail in Sect. 3. The major theoretical physics aspects of a reactor plasmld are summarized in sect. 1 .

The entire torus is enclosed in a concrete moat which provides a structural foundation for the reactor components and remote maintenance equipment, and a biological shield. Figure 3 shows a cross section of the torus and moat at an early stage of disassembly.

The high aspect ratio EBTR configuration affords several design options in the remaining areas. Two blanket and magnet shield concepts have been developed. The first blanket uses a stainless steel structure, natural lithium for breeding, and a eutectic nitrate salt for coolant. The associated shield materials are stainless steel and borated water. The shield is cooled by circulation of the borated water. The second design includes a Nb-1\% Zr first wall, a stainless steel structure, and 
natural lithium both for breeding and as the coolant. Electromagnetic pumps are used to circulate the lithium. The magnet shield in this case is composed of concrete loaded with austenitic iron shot ( $40 \%$ by volume), and is cooled by $\mathrm{CO}_{2}$.

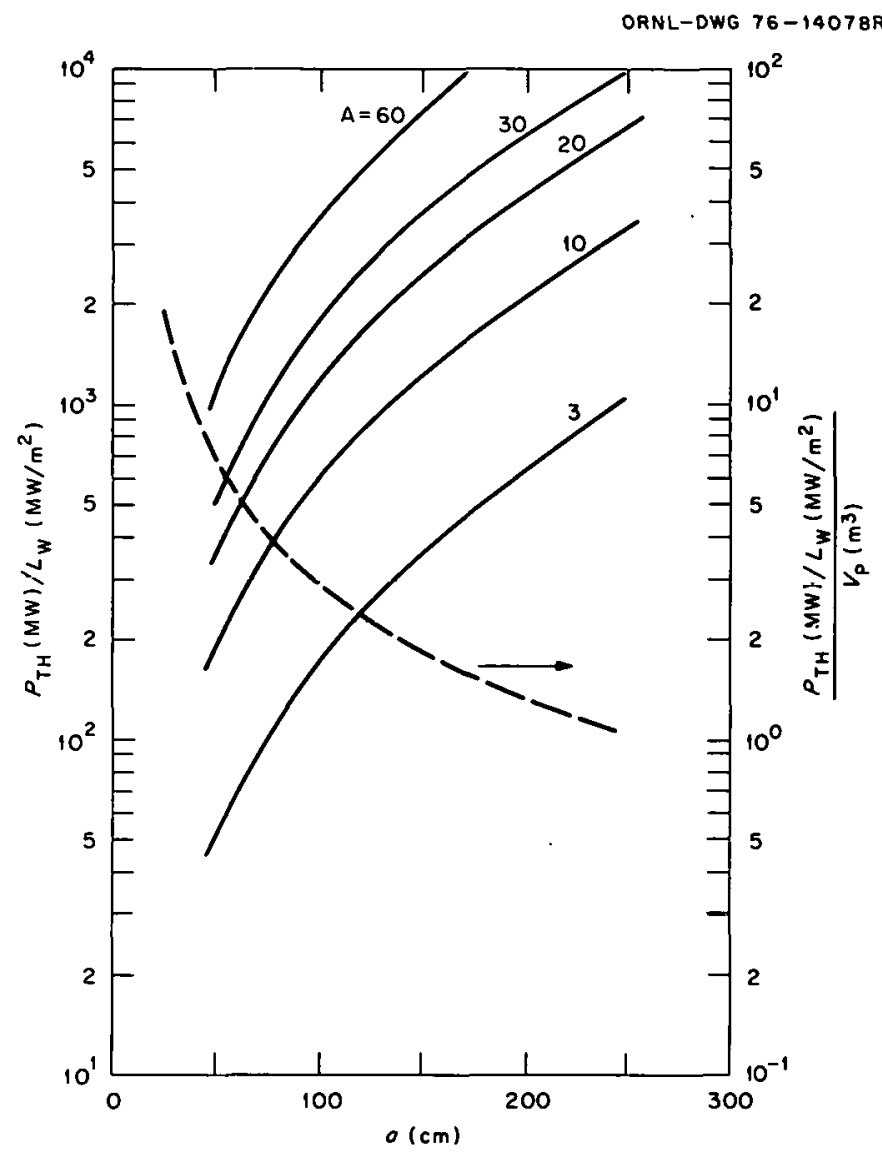

Fig. 2. Relationships between geometrical and power production parameters.

Two superconducting magnet alternatives have been examined. In both cases the magnetic field and coil loading are nearly symmetrical. There is no need to use D-shaped or oval-shaped coils to minimize bending moments so coils with a circular shape are iused. The first magnet system option has 48 Nbili superconducting magnets operated under pool-boiling conditions. The average current density in the wiridings is $1550 \mathrm{~A} / \mathrm{cm}^{2}$, which results in a peak field strength of $7.3 \mathrm{~T}$ in the winding and $4.5 \mathrm{~T}$ at

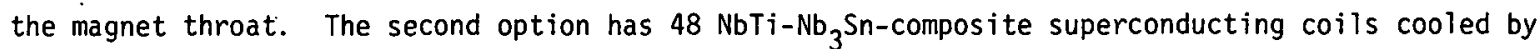
supercritical helium under forced-flow conditions. The peak fields in the $\mathrm{Nb}_{3} \mathrm{Sn}$ and $\mathrm{NbTi}$ windings are $10 \mathrm{~T}$ and $5.2 \mathrm{~T}$ respectively. The average current density in the high field windings is $2000 \mathrm{~A} / \mathrm{cm}^{2}$; in the lower field windings it is $4000 \mathrm{~A} / \mathrm{cm}^{2}$. Both options are fully stabilized and include current dump protection circuitry in case of a magnet failure. More discussion on magnet design and scaling is given in Sect. 5.

Since impurity production and behavior in the reactor cannot reliably be predicted with certainty and since these will be critical to achieving ignition and steady state operation, this area was treated conservatively. In one case a system with a toroidal divertor was studied. Energy 
and particle fluxes are accommodated by the divertor. Alternatively, a case was studied for which it was assumed that impurities are controlled but the full energy flux from the plasma must be tolerated by the first wall. The latter system without divertor has significantly more stringent design criteria for the first wall thermal hydraulic system since it must handle $20 \%$ of the total thermal output power and $\sim 8 \%$ of the neutron energy. Characteristics of the divertor system are summarized in Sect. 6 .

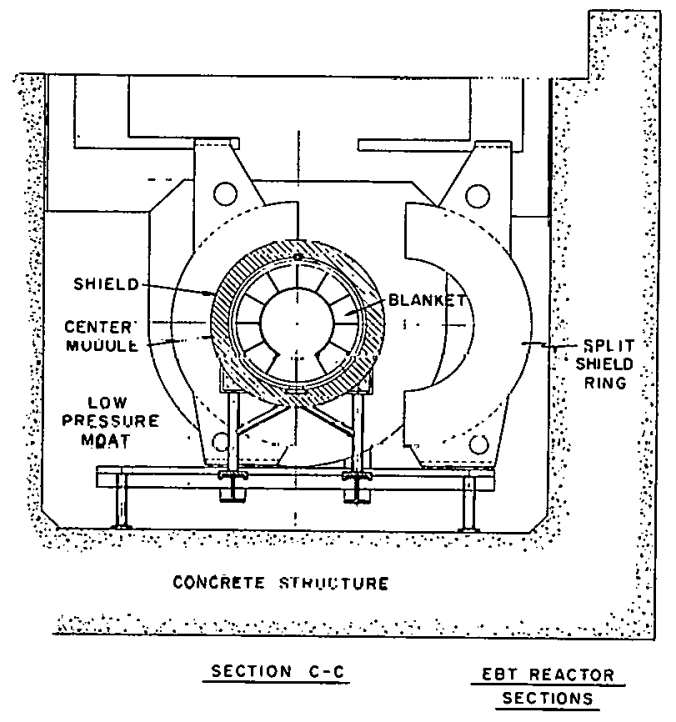

Fig. 3. Cross section view of the torus and moat.

There are two structural arrangement options. (See Sect. 7.) In either, the torue is modular to facllitate asseribly and remote maintenance. For the movable magnet concept, the magnets and modules are equal in number. Each module includes the first wall, blanket, magnet shield and the magnet itself. The entire unit, which weighs about 600 tons, can be removed to a hot cell for maintenance. In the fixed magnet concept, the number of modules is twice the number of coils. The modules located unider the magnets, which include the first wall, the blanket, and the magnet shield, are not identical to those located between the magnets. Thus there are two sets of 48 identical modules in the reactor. The fixed magnet concept permits any module to be removed for maintenance without disturbing the superconducting coils. The magnet is removed only if it fails. It is important to realize that either approach allows standardization of the major EBTR components. That is. standard magnets and modules can be fabricated and assembled into toroidal configurations of different major radii. Forty=eight coils are needed when the major radius is $60 \mathrm{~m}$. The minimum size device which is compatible with the standard module concept has a major radius of $30 \mathrm{~m}$ and requires 24 coils. The power output in the latter case is $1775 \mathrm{MW}(\mathrm{th})$ for a neutron wall loading of $1 \mathrm{MW} / \mathrm{m}^{2}$ and can be $5300 \mathrm{MW}(\mathrm{th})$ if $3 \mathrm{MW} / \mathrm{m}^{2} \mathrm{can}$ be tolerated. Standardization has broad implications for commercialization.

Preliminary neutronics analyses of the blanket options discussed earlier have been carried out using the one-dimensional discrete ordinates code ANISN to assess the nuclear performance of the two proposed blanket shield designs. The ability of the blanket assemblies to recover the kinetic energy of the fusion neutrons and secondary gamma rays in the form of heat, to breed tritium, and to reduce 
the radiation which is incident on the toroidal magnet coils were used to evaluate the nuclear

performance. Results of the analyses are summarized in Sect. 8 .

TABLE 1

EBT Reactor Reference Parameters

\begin{tabular}{|c|c|c|c|}
\hline . & EBTR- $48^{\mathrm{a}}$ & \multicolumn{2}{|c|}{ EBTR-24 } \\
\hline Plasma radius, a $(\mathrm{m})$ & 1.0 & \multicolumn{2}{|c|}{1.0} \\
\hline Aspect ratio, $A$ & 60 & \multicolumn{2}{|c|}{30} \\
\hline Major radius, $R_{0}(\mathrm{~m})$ & 60 & \multicolumn{2}{|c|}{30} \\
\hline Mirror ratio, M & 1.78 & \multicolumn{2}{|c|}{1.78} \\
\hline Ion temperature & 15 & \multicolumn{2}{|c|}{15} \\
\hline Ion density, $N_{j} \times 10^{-20}$ & 1.2 & 1.25 & 2.13 \\
\hline Beta, $\beta(\%)$ & 25 & 24 & 42 \\
\hline Magnetic field on axis, $B_{T}(T)$ & $2.5-4.5$ & \multicolumn{2}{|c|}{$2.5-4.5$} \\
\hline Number of coils, $\mathrm{N}$ & 48 & \multicolumn{2}{|c|}{24} \\
\hline Power, $P_{\text {th }}$ (MW) & 4000 & 1775 & 5300 \\
\hline Power density, $\mathrm{P}_{t h} / \mathrm{V}_{\mathrm{p}}\left(\mathrm{MW} / \mathrm{m}^{3}\right)$ & 3.37 & 3.0 & 9.0 \\
\hline Neutron wall Toading, $L_{W}\left(M W / m^{2}\right)$ & 1.13 & 1 & 3 \\
\hline Cold zone, $\delta(m)$ & 0.2 & \multicolumn{2}{|c|}{0.2} \\
\hline Blanket and shield thickness, $t_{s b}(m)$ & 1.75 & \multicolumn{2}{|c|}{1.75} \\
\hline Coil inner radius, $r,(m)$ & 2.95 & \multicolumn{2}{|c|}{2.95} \\
\hline Current density, $J_{C}\left(A / \mathrm{cm}^{2}\right)$ & 1500 & \multicolumn{2}{|c|}{1500} \\
\hline Coil radial thickness, $t_{c}(m)$ & 0.71 & \multicolumn{2}{|c|}{0.71} \\
\hline Coil half length, $L / 2(\mathrm{~m})$ & 1.30 & \multicolumn{2}{|c|}{1.30} \\
\hline
\end{tabular}

\section{PLASMA ENGINEERING}

Plasma engineering uses the results from applied physics analyses to design fusion systems. The design process in EBTR is difficult since some of the critical aspects of the system and its behavior are not well understood at present. It is necessary to make plausible estimates of the parameters and to retain flexibility so the design can accommodate new results from theoretical and experimental programs. This approach generates design criteria which define the appropriate directions for engineering and technology development efforts.

The fundamental plasma and device characteristics, system economics, and technology considerations for a commercial reactor of about $4000 \mathrm{MW}(\mathrm{th})$, exclusive of blanket multiplication, have been studied (see Appendix A). The neutron wall loading has been restricted to values near $1 \mathrm{MW} / \mathrm{m}^{2}$. Although modest increases in wall loading improve system economics, loadings above 3 or $4 \mathrm{MW} / \mathrm{m}^{2}$ will almost certainly be unoconomical due to the reliability and pumping penalties. 
The plasma size is determined by specifying power output, power density, and neutron wall loading. Plasma dynamics simulations, equilibrium, and drift orbit calculations show that a plasma radius of about $1 \mathrm{~m}$ is adequate to attain ignition, assuming neoclassical and/or classical scaling. However, no definitive basis for the size required for the reactor plasma has been developed. As in the other system studies, size and plasma parameters are determined self-consistently from theoretical models, but the ultimate values will be determined by experimental results which validate the assumptions used or provide the basis for changing them.

\subsection{System Characteristics}

The first material boundary surrounding the plasma is assumed to be capable of tolerating a neutron wall loading, $L_{w}$, on the order of 1 to $3 \mathrm{MW} / \mathrm{m}^{2}$. This boundary exists at a distance from the plasma center equal to the average plasma radius, $a$, plus the cold zone, $\delta$. The total fusion thermal power output (exclusive of blanket multiplication), $P_{t h}$, produced in the plasma is

$$
P_{t h} \cong \frac{17.6}{14.1} L_{W}(2 \pi)^{2} A a^{2}(1+\delta / a)
$$

where $A$ is the plasma aspect ratio.

The wall loading is coupled to the thermal power density, which in turn will dictate the desirable characteristics for the plasma. For example,

$$
\frac{P_{t h} / L_{w}}{V_{p}} \cong \frac{17.6}{14.1} \frac{2}{a}(1+\delta / a),
$$

where $V_{p}$ is the plasma volume. Note that for an assumed wall loading, the thermal power density is independent of $A$ and depends only on $\delta$ and $a$. The self-consistent relationships between the genmet.rical and power production parameters are shown in Fig. 2.

The plasma power density required to attain a particular wall loading in a given size device has been determined, and dictates the range of plasma densities and temperatures required to produce the power density. That is,

$$
\mathrm{P}_{t h} / V_{p} \simeq 2.8 \times 10^{-18} \frac{\mathrm{N}_{\mathrm{i}}^{2}\langle\sigma v>}{4},
$$

where <ov> is the fusion reaction rate probability and $N_{D} \simeq N_{T} \simeq N_{i} / 2$ has been assumed. The power density can be written in terms of beta $(\beta)$, the ratio of plasma pressure to magnetic energy density, and $B$, the magnetic field strength:

$$
\mathrm{P}_{t h} / V_{\mathrm{p}} \simeq 2.8 \times 10^{-18} \frac{<\sigma \mathrm{V}>}{4 \mathrm{~T}^{2}} \frac{\beta^{2} \mathrm{~B}^{4}}{(4 \mu)^{2}}
$$

The trade-offs between $B$ and $B$ must be considered. High- $B$, low-B operation is desirable. The practical minimum magnetic field in this work is taken to be $B \sim 2.5 \mathrm{~T}$.

In addition to the magnetic and plasma physics constraints described above, the required microwave frequencies must be determined. A magnetic field of $\sim$ 4.0-4.5 T exists near the magnet throat, where the resonant frequency. is $\sim 120 \mathrm{GHz}$, suitable for background plasma heating. Because of the decreased density and field strength in the region of the stabilizing annuli, microwave frequencies less than this by a factor of 2 may be acceptable to sustain the annuli. A final decision on the means of heating the EBTR plasma should not be made now but the frequency requirements, if microwaves are used, are compatible 
with those of components under development. It is more likely, in view of cost, complexity, and efficiency criteria, that an optimized system will utilize microwaves to sustain the annuli but use neutral beam injection to heat the plasma to ignition. To test the plausibility of beam heating, a plasma point kinetic model (see Appendix D) was used in a computer study of various start-up scenarios. For the particular case studied in Appendix D, it was determined that $200 \mathrm{MW}$ of neutral beam power (150 keV) would be sufficient to heat EBTR-48 to ignition in about $3 \mathrm{sec}$. The value of $200 \mathrm{MW}$ is probably an overestimate since longer start-up times may require smaller amounts of. input power. Full penetration calculations have not been completed. The estimates in Appendix $D$ serve to demonstrate feasibility, but are not sufficiently detailed for optimization studies.

\subsection{System Economics}

Several conclusions can be drawn from economic analysis of an EBTR. Although absolute component costs are not well known, the economic analysis indicates that an EBT reactor should be an ignition device and specifies limits on the feedback power which would be available to sustain the plasma conditions. In the simplest case the sustaining power is that necessary to drive the stabilizing annuli.

The effect on capital cost of the physical dimensions of the reactor is investigated through trade-off analysis (see Apdendix $A$ ) to determine how a reduction in reactor size would reduce the cost of electricity.

\section{PLASMA PHYSICS}

The plasma physics areas which determine the EBTR operating characteristics are: (1) particle orbits and their effects on efficient use of the plasma chamber volume, (2) plasma stability and equilibrium, and (3) scaling, transport, and modeling, which are described at length in Appendixes B-D.

For a high beta, steady state system, the plasma equilibrium, particle orbits, ambipolar electric field, and transport phenomena are closely coupled to one another, and an adequate treatment of any one requires consideration of the others. The interrelations are: (1) equilibrium magnetic fields depend on the plasma pressure profile, (2) guiding-center drift orbits depend on the equilibrium magnetic field and the ambipolar electric fields, and (3) transport rates, which together with energy sources and sinks determine the profile, depend on the guiding center drift orbits. A self-consistent treatment of this coupled set of problems for a large-scale EBTR, while being vigorously pursued, is not yet available. Intermediate and/or partial answers have been used to proceed into the full self-consistent treatment.

\subsection{Equilibrium and Drift Surfaces}

The requirement for efficient use of the volume within the vacuum chamber of the reactor proved to be very important to the design. The shield and blanket which surround the plasma chamber in the reactor make efficient field usage more difficult than in the present experimental device (EBT-I) ${ }^{3}$

Finite beta and ambipolar electric fields make considerable differences in the particle orbits. For the reference design (EBTR-48), a detailed calculation was carried out for a sample equilibrium, guided by the experimental observation that the ambipolar electric field is strongest in the vicinity of the annuli. The spatial position of the hot electron annuli is critical to obtaining efficient utilization of the volume within the vacuum chamber. Macroscopic stability demands that the toroidal core plasma pressure be relatively constant near the minor axis and fall rather rapidly in the region of the annuli, so the annuli form a set of "guard rings" which surround the toroidal core. Experimental

observation and numerical computation show that the annuli form near contours of constant $\left|\vec{B}_{\text {vacuum }}\right|$ in the midplane of the device. Toroidal effects cause these contours to be shifted inward toward the 
major axis. To the lowest order, the plasma follows the field lines, and for the present inner wall design some of the field lines which pass through the coil aperture intersect the lateral wall of the chamber, so the present wall design limits the diameter of the annuli. This problem can be solved by "bulging" the inner wall near the midplane (as in EBT-I) and using a nonuniform shield and blanket thickness within the coil throat. In EBTR-48, the shield is sufficiently thick that a radial shift will not result in a significant change in coil heat loading or radiation damage. An alternative solution is to increase the coil radius by about $25 \mathrm{~cm}$ while keeping the plasma location and the thickness of the blanket and shield constant. Either method would lead to the plasma size required for EBTR-48 and would correspond to a usable volume of about $100 \%$.

There are three features which are relatively independent of the details of the inner wall design and the magnetic equilibrium and ambipolar electric fields: (1) low energy particles are well confined, (2) mirror trapped particles are well confined, and (3) high energy particles with $v \mathbb{v} v \sim 1$ are not well confined. The use of supplementary coils can improve the confinement of this last class of particles.

\subsection{Macroscopic Stability}

The viability of EBT as a reactor requires the stable confinement of a plasma with a density of about $103 \mathrm{~m}^{-3}$ and a temperature on the order of $10-15 \mathrm{keV}$. A relativistic hot electron population (annuli) with $T \sim 100 \mathrm{keV}$ is crucial to the stability of the EBT plasma. The annulus density, however, is only about $10^{18} \mathrm{~m}^{-3}$, a small fraction of the total electron density. The hot electron population is immune to the macroscopic flute-like drift instabilities because of the fast drift velocities and large Larmor radii of its particles. By modifying the magnetic field, the annuli also provide stability for the rest of the plasma, which is composed of warm ions and electrons. Thus the simplest model for discussing the stability of the EBT plasma is one with three components: hot electron annuli, warm electrons, and warm ions. One can treat first the instabilities of the hot electrons, and then the instabilities of the warm species. The efiort to date has been concentrated on macroscopic instabilities, those which produce large particle tránsport and would be tàtál tó a réactór.

\section{Stability of the hot electron annuli}

The requirement for stability is that the temperaiure of the hot electrons be high enough and the magnetic field gradient produced by diamagnetism be large enough to achieve a $\nabla B$ drift frequency which exceeds the ion cyclotron frequency. When this condition is satisfied, the viasov-Maxweli equations show that hot electron drift waves are stabilized, apparently by interference between the responses of the fast drifting electrons and the slowly drifting ions. ${ }^{5}$ As the hot electron density or temperature is reduced, these waves become unstable, and at still lower temperature, where the magnetohydrodynamic (MHD) and quiding center theories are applicable, they become the unstable fiutes and ballooning interchanges predicted by these theories. Thus, stable operation of a reactor will require that the hot electrons achieve a temperature comparable to that in the present device (EBT-I) and a pressure profile of sufficient steepness to produce large $\nabla B$ drifts. Near the outer edge of the annuli, the hot electron temperature is not high enough to stabilize drift waves. However, the outer edge of the plasma can be line-tied, which effectively stabilizes these modes. Present theory does not allow a definitive answer on how large the line-tied region must be, nor how line tying will be affected by the addition of a divertor system. 


\section{Stability and beta limitations of the bulk plasma}

In the vacuum field of EBT, the bulk plasma is likewise subject to unstable drift and MHD modes at frequencies characteristic of their $\nabla B$ and diamagnetic drifts. The hot electron annuli stabilize these modes by a very interesting mechanism. The hot electron diamagnetic currents act as "invisible coils" colocated with the bulk plasma to create a region of minimum average $B$ in which $6 \mathrm{~d} \ell / B$ decreases with radius. In this region a stable bulk plasma can be established and then stably extended to the magnetic axis, even though the minimum average $B$ region does not extend to the magnetic axis. Figure 4 shows the location of the hot electron plasma, the region of decreasing $\sigma d l / B$, and the stably confined bulk plasma. The bulk plasma $\beta$ can approach that of the annuli before instabilities occur.
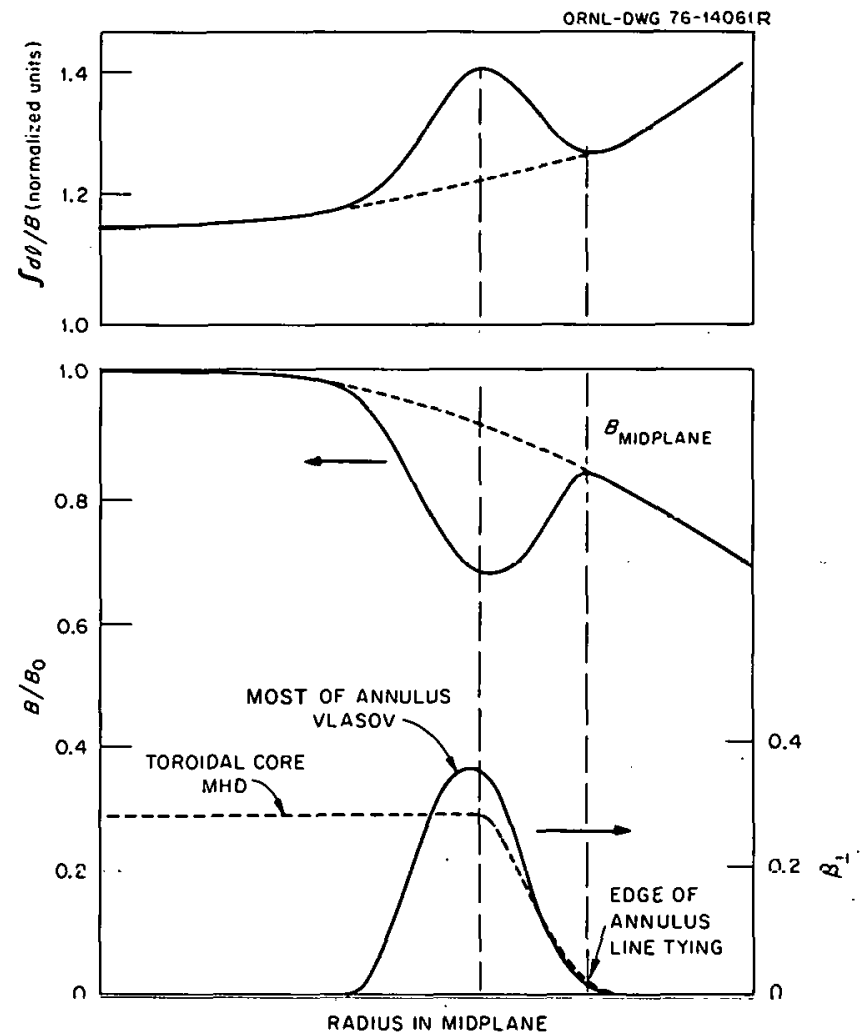

Fig. 4. $\emptyset d l / B$, magnetic field, and pressure vs distance from the minor axis in the midplane for the vacuum field (dashed) and the finite $B$ field (solid).

Once a magnetic well has been established by the annuli (at annuli $\beta \sim 5-15 \%$ ), a $\beta$ of the bulk plasma comparable to, or even somewhat higher than, the $\beta$ of the annuli can be achieved which is stable to all modes encompassed by guiding center or ideal MHD theories. The modes, which limit bulk $B$, are pressure-driven ballooning interchanges.

Experimental confirmation of these optimistic $\beta$ predictions is not possible in the present device because there $\beta$ is limited by heating and transport considerations. However, the lower threshold for stable confinement at annuli $B \sim 8 \%$ is observed, where it marks the boundary between the C- and T-modes. ${ }^{6}$ 


\subsection{Scaling, Transport, and Modeling}

The design of EBT fusion reactors must be based on scaling laws which have not been fully verified by experiments. However, in the observed macrostable regime of operation in EBT-I ${ }^{6}$ there is a reasonable agreement between experiment and a simplified neoclassical point model. The EBT-S and EBT-II ${ }^{3}$ devices are designed to allow further study of transport and scaling properties, especially in the collisionless regime (of interest to reactor operation). Theoretical work in support of these experiments will refine the existing transport theories.

In view of the uncertainties in the theoretical coefficients and the present lack of experimental evidence in this area, the plasma energy balance was modeled using both neoclassical and classical theory with appropriate density and temperature scale lengths (see Appendix D). The reactor plasma model is consistent with the theoretical physics model: a toroidal core with nearly uniform density and temperature within the plassma radius. Which is determined by the stabilizing annuli. Flexibility was retained in the simulation models to permit updated data to be incorporated as they become available.

\section{TOROIDAL MAGNET SYSTEM DESIGN AND SCALING}

The EBTR magnet system designs are determined by considerations of scaling, conductor design, coil design, cryogenics, and protection. Aside from the plasma constraints, the main goal was the design of coils which could be built with existing technology or with technology which is the immediate goal of existing development programs.

A description of an alternate magnet design is also given. This design is based on the high current density, forced-flow, bundled conductor concept. This approach holds great promise for the future. Main features of both designs are discussed in detail in Appendix $E$.

\subsection{Maymet S:alliny}

Independent of plasma physics considerations, the number of coils, mirror ratin, coil radius, and the reactor major radius can ali be related by the geometry of the vacuum magnetic fields. Plasma physics considerations further relate the allowable mirror ratios to the aspect ratio, electron ring beta, stable plasma volume, magnetic axis shift, and similar quantities.

\subsection{Conductor Design}

It is generally agreed that superconducting magnets are required for an economical fusion reactor. Reactor application also demands high reliability, requiring a fully cryostahilized r.nndurtar design. Multifilamentary $\mathrm{Nb}_{3} \mathrm{Sn}$ is at an early stage of development, so presently available monolithic composites of multifilamentary $\mathrm{NbTi}$ in Cu matrix are used in the reference reactor study. The average current density in the windings is $1500 \mathrm{~A} / \mathrm{cm}^{2}$, which gives a peak field strength in the winding of $7.3 \mathrm{~T}$. The

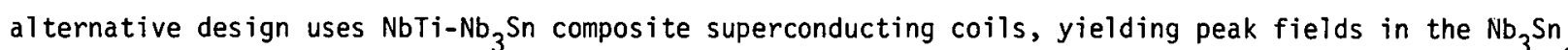
and NbTi windings of $10 \mathrm{~T}$ and $5.2 \mathrm{~T}$ and corresponding average current densities of 2000 and $4000 \mathrm{~A} / \mathrm{cm}^{2}$.

Cryostability is achieved by soldering the composite to formed copper strips with punched slots. There are sufficient cooling surfaces to transfer up to six times the Joule heating produced if all the current were to flow in the copper,

\subsection{Magnet Design}

In contrast to the tokamak, the EBT has a high aspect ratio, so the magnetic field and loading are nearly symmetrical and there is no need to use D-or oval-shaped coils to minimize the bending moments. Circular coils are used in EBTR-48 and EBTR-24. 
The coils are fairly long compared with their radial thickness, and their axis is horizontal. Pancake-wound construction with spaces between pancakes is chosen for its high voltage capability and short cooling channels.

Natural convection pool boiling is chosen as the cooling method for the reference design, while the alternative design option operates under forced-flow conditions. Coolant can pass along the conductor through the copper strip fins or through the slots, resulting in a flow in both radial and azimuthal directions. This allows flow mixing and aids helium bubbles to reach the top of the coil, an important feature for a coil with a horizontal axis.

An interesting possibility is that the magnets may be made modular and used in reactors of different sizes. As long as the ratio of major radius to the number of coils is fixed, the field strengths produced by modular magnets of the same size are not sensitive to the size of the reactor. The mirror ratio and coil bore fixes the length of the blanket and shield modules which are located between adjacent magnets. These parameters are fixed by plasma physics and blanket-shield engineering requirements. Furthermore, for given neutron wall loading, the plasma radius is relatively insensitive to changes in the major radius of the machine. The various components of the reactor system can be modular and standardized and still serve in reactors of different major radii and power outputs.

\subsection{Cryogenics}

The toroidal magnets in the EBTR will be cooled by liquid helium under pool boiling conditions for. the reference case and by supercritical helium under forced-flow conditions for the alternate case. Individual dewars and vacuum vessels are used for each coil. To avoid intereference with the remote assembly of the blanket, helium will be supplied to the magnets in the radial direction from the cold box.

The EBT reactor is expected to operate in steady state. Accordingly, there will be no heat load requirement due to pulsed fields. The heat loads that must be removed by the liquid helium (or supercritical helium) cryogenic system include the radiation heat absorbed from the magnet dewar surface, the loads due to conduction through the support and instrument leads, and the radiation energy absorbed from incident neutrons and gamma rays.

\subsection{Protection}

High operating current $(25 \mathrm{kA})$ is used to reduce terminal voltage during discharge, and four conductors (each carrying $6.25 \mathrm{kA}$ ) are connected in parallel to supply the total current. To ensure even distribution of current, a spiral winding technique is adopted.

Voltage taps are used on each coil as the main quench detection device. Pick-up. coils on the current leads of the power supplies can be used to compensate for the inductive voltage. External dump resistors are used as heat sinks.

\section{DIVERTORS}

Divertors may be required in fusion reactors to provide for the removal of plasma reaction products, to act as conduits for steady state removal of diffusing plasma, to serve as access for initial pumpdown, and to keep atoms sputtered from the system boundaries from diffusing deeply into the plasma. The high aspect ratio and relatively low magnetic field of EBT make it possible to design a.relatively simple "classical toroidal divertor." (See Appendix F.) 


\subsection{Divertor Requirements}

The magnetic field geometry of the divertor must be such that the separatrix can be accurately placed at the plasma boundary with relatively small field perturbations inside the $e^{-1}$ imiting radius. Field curvatures should be as gentle as possible out-ide the separatrix. The diverted field lines in the scrape-off layer assure that the escaping plasma energy is uniformly distributed over the surface that intercepts $i t$. The region in which the divertor field lines penetrate the first wall should be as small as possible to minimize any chance of gas back-streaming into the plasma and to avoid excessive neutron streaming into and through the divertor. Divertors must also have sufficient heat transfer area to handle full steady state overloads in an emergency plasma shutdown.

The forces on the divertor will be large, so the design must provide mechanical support and access, be compatible with the standard EBT module and be capable of staged multiple use so that three of the four divertors in the reference design would be capable of handling the total load.

\subsection{Divertor Field Design}

In the EBT divertors described here, field reversal is accompiished by reversing the current in one of the standard coils. The reversed current required in this coil is less than the standard current by a factor of 1.8-2. For the same overall current density, the coil can be smaller than one of the standard coils. To maintain the field intensity at the plasma center line under the reversal coil, two additional "shaping" coils are required. These are positioned on either side of the reversal coil and approximately one-quarter to one-half of the reactor section length away from the reversal coil. The current in each of these is about one-half of the current in the reversal coil. In addition, a set of low current "tickling coils" with radii about 2-2.5 times that of the standard coil radius are used for the separation of diverted field lines.

For reactors with larger major radii, the divertor design will be easier and effective use will be made of the available volume; the design of an EBTR divertor does not seem to present any major difficulties.

\section{3 Divertor Moschanical Design}

Four divertors are equally spaced around the reactor, designed so that any three of them will be capable of handling the power recovery (see Fig. 1). The wall loading for three operating divertors would be about $0.4 \mathrm{MW} / \mathrm{m}^{2}$, well below the limits imposed by heat transfer to the liquid lithium coolant. A possible cryopump system for reactor pumpdown and reaction product removal is considered. Eight such systems (two for each divertor) will be sufficient to maintain a base pressure of $10^{-7}$ atm with a cryopumping speed of $1.7 \mathrm{l} / \mathrm{cm}^{2}-\mathrm{sec}$, well below currently available cryopumping capacities.

\section{MECHANICAL DESIGN}

The EBT reactor study has considered the mechanical design of the following: (1) the first wall, (2) the nuclear blanket, (3) the radiation shield to protect the coils, (4) the superconducting coil support, and (5) the concrete enclosure and biological shield. The design was carried to sufficient detail (see Appendix G) to demonstrate fabrication and remote maintenance. The stresses will be within acceptable limits and the required heat removal will be possible.

Since the EBTR has a high aspect ratio and relatively wide magnet spacing, it is possible to design one module that can be used in machines of various sizes. The module is composed of the first wall, blanket, shield, and coil. In the case of a reactor with a 30-m major radius, 24 modules are 
required. The number of modüles in a larger device increases in proportion to the major radius so that the spacing of the magnets remains constant. This concept of standardized modules is extremely favorable in the context of a fusion reactor economy.

Two fabrication approaches have been studied. In the first, there are twice as many modules as magnets. A standard module is located under each coil and a module is located between each pair of adjacent coils. Removal of the spacer modules (between coils) permits the less accessible modules to be rolled axially into position for vertical removal from the machine. This procedure permits maintenance to be performed with the coils in place and at $4.2^{\circ} \mathrm{K}$. In the second approach the number of magnets and modules is equal. Maintenance is performed by removing the entire blanket-coil unit. This feature is combined with other differences relative to the reference design: (1) lithiumcoolant is used, (2) electromagnetic pumping is employed, and (3) the magnet shield is made of concrete which contains $40 \%$ iron shot by volume. The two designs are referred to as the fixed magnet and movable magnet concepts.

The blanket and first wall designs differ in the movable and fixed magnet concepts. The primary differences are in the structural material and the method of heat removal. In the fixed magnet case the bianket is contained in a set of stainless steel compartments which surround the plasma and are filled by a stagnant lithium pool, a canned graphite reflector, and stainless steel slabs for gamma-ray shielding. The blanket is cooled by a nitrate eutectic salt which is circulated through stainless steel tubes. The salt enters the blanket at $260^{\circ} \mathrm{C}$ and exits at $485^{\circ} \mathrm{C}$. The blanket and first wall absorb about $96 \%$ of the energy produced by the plasma.

The movable magnet concept is fabricated from Nb-1\%Zr and uses lithium as the coolant. The EBT reactor configuration may permit the use of lithium, which has the advantages of superior heat transfer and neutronic characteristics. The disadvantage of the high pressure drop due to MHD effects requires particular attention. The pressure drop in an inlet or outlet plenum is about 120 psi. This imposes high stresses on the plena and the first wall, so electromagnetic pumps are recommended for use in the plena. The first wall in this concept is an integral part of the blanket and has a common lithium coolant circuit with it. In the fixed magnet case, a totally separate first wall is employed with its own cooling circuit. The maximum temperature in the wall is about $365^{\circ} \mathrm{C}$ so that the wall lifetime for radiation damage should be acceptable.

The superconducting coils must be shielded from the radiation produced in the plasma and blanket. In the fixed magnet case, the shield, which is $53.5 \mathrm{~cm}$ thick, is composed of concentric cylinders; the annular spaces are filled with stainless steel spheres, lead, and borated water. The borated water circulated to cool the shield enters at $35^{\circ} \mathrm{C}$ and exits at $90^{\circ} \mathrm{C}$. The movable magnet concept shield is $86 \mathrm{~cm}$ thick and is composed of cylindrical stainless steel shells filled with concrete mixed with austenitic iron shot ( $40 \%$ by volume). The shield is cooled by $\mathrm{CO}_{2}$. This shield concept has several advantages and will be studied further for possible application in the fixed magnet case.

The magnet support structure differs in the two designs. In the movable magnet case, each coil is supported and constrained by 16 tendons which are connected to a support ring. The ring is attached to the concrete moat which encloses the reactor. The overall module is positioned and secured to its foundation by wedges which are attached to the corners of the magnet dewar. The fixed magnet concept uses cables to support the gravity loads and the centering forces, so the dewars can completely enclose the magnets except that bellows seals are used where the cables penetrate the dewar wall. The cables give a uniform stress on the coil bobbin and maintain a uniform load distribution during magnet cooldown. This scheme is restricted to the fixed magnet case since the cable would make coil removal difficult.

A concrete moat with articulated roof slabs encloses the torus. The moat is about $15 \mathrm{~m}$ wide and $18 \mathrm{~m}$ high with a wall thickness of $3 \mathrm{~m}$. In the fixed magnet design, concrete abutments are lociated 
on the inner side of the walls adjacent to each coil. These are used to restrain lateral movement of the coils in case of a quench. The roof slabs over the coils are used to support the coil and module gravity loads. Modules between the coils are supported from the moat floor. In the movable magnet concept, gravity loads are supported from the floor.

In sumary although the fixed and movable magnet concepts differ in numerous respects, an ultimate design may include features from both of them. The important point here is that the mechanical problems related to the EBT reactor can be solved. The high aspect ratio, reduced forces, and lack of pulsed magnetic fields make the EBT reactor very attractive from the engineering point of view.

\section{NEUTRONICS}

Preliminary neutronics analyses for the EBTR designs have been carried out using the one-dimensional discrete ordinates code ANISN ${ }^{7}$ to assess the nuclear performance of two proposed blanket-shield designs. The capability of the blanket assemblies to recover the kinetic energy of the fusion neutrons and secondary gamma-rays in the form of heat, to breed tritium, and to reduce the radiation incident on the toroidal magnet coils, were evaluated. Two blanket and shield configurations are used. The first (or reference) design utilizes stainless steel 316 (SS-3I6) as the first wall and structural material. The blanket assembly consists of alternating layers of lithium and graphite followed by a gamma-ray shield which consists of alternating layers of SS-316 and lithium coolant channels. The total thickness of the lithium is $41.92 \mathrm{~cm}$ and the graphite moderator-reflectors have a combined thickness of $15 \mathrm{~cm}$. The main constituents of the shield are borated water (35\%) and SS-316 in the form of spheres (65\%). A $12-\mathrm{cm}$-thick lead liner surrounds the shield and reduces the gamma radiation incident on the magnet coils.

The second (or alternate) blanket and shield option utilizes niobium as the first wall material. The remainder of the structure is SS-316. The total thickness of lithium in this design is $54.5 \mathrm{~cm}$. A $15-\mathrm{cm}$-thick graphite reflector-moderator follows the lithium region. In this design, the main constituent of the shield is heavy concrete consisting of $40 \%$ iron and $60 \%$ ordinary concrete. A 5 -cmthick lead liner surrounds the assembly.

In both designs, natural 1 ithium $\left(7.4 \%\right.$ of ${ }^{6} L i, 92.6 \%$ of $\left.{ }^{7} \mathrm{Li}\right)$ is the tritium breeding material. The magnet coils were taken to have the same composition as those considered in the EPR neutronics analysis. ${ }^{8}$

Some of the results are given in Table 2. In the design utilizing niobium as the first wall, the tritium breeding ratio is 1.614 compared to 1.353 for the design utilizing stainless steel as the firct. wall. However, the tritium breeding ratio per cubic centimeter is essentially the same in both designs. The energy deposition rate in the first winding of the magnet coil is also given. The concrete shield option is more effective in reducing the nuclear heating in the first winding of the magnet coil. The heavy concrete shield is $86 \mathrm{~cm}$ thick compared to $53.5 \mathrm{~cm}$ for the shield of borated water plus SS-316. The lead liner in the reference design is $12 \mathrm{~cm}$ thick, compared to $5 \mathrm{~cm}$ in the alternate design. The radiation damage to the first wall materials is also given in Table 2 . 
TABLE 2

Calculated Nuclear Performance for Two EBTR Reference Options

Tritium Breeding Ratio

(tritium nuclei/incident neutron)

${ }^{6}$ Li
${ }^{7}$ Li
Total

Stainless Steel Salt Coolant

0.855

0.498

1.353
$\mathrm{Nb}-1 \% 2 \mathrm{r}$, Stainless Steel

Lithium Coolant

0.881

0.733

1.614

Nuclear Heating in the First Magnet Coil Wiriding

$\left(\mathrm{W} / \mathrm{cm}^{3}\right) /(\mathrm{n} / \mathrm{s} / \mathrm{cm})$

neutron
gamma ray
Total

Radiation Damage to the First Wal1

Displacements per atom/year ${ }^{a}$

10.71

13.14

Hydrogen gas production (appm/yr)

444.38

127.2

Helium gas production (appm/yr)

131.21

27.2
$2.96 \times 10^{-25}$
$2.52 \times 10^{-24}$
$2.78 \times 10^{-24}$

${ }^{\mathrm{a}}$ Based on effective displacement energies of $40 \mathrm{eV}$ for SS-316, $60 \mathrm{eV}$ for $\mathrm{Nb}$.

\section{CONCLUSIONS}

An EBTR reference design has been developed. If the present understanding of the physics extends to the reactor regime, the system will operate at high beta and high power density, and in steady state. Problems of accessibility, structural design, and remote maintenance are eased considerably because of the high aspect ratio in the EBTR and the fact that there are no pulsed magnetic fields.

The flexibility afforded by the EBTR configuration leads to several options in the areas of materials, primary coolant, blanket and shield arrangements, superconducting magnets, and maintenance procedures. When an EBTR reference design is envisioned which uses the more conventional options, it is found that the reactor could be constructed using techniques and technologies which exist or are the objectives of present development programs. The other more advanced design options require longer range development but lead to a potentially more attractive reactor plant.

\section{REFERENCES}

1. J. F. Clarke, High Beta Flux-Conserving Tokamaks, ORNL/TM-5429, Oak Ridge (June 1976).

2. R. A. Dory and Y-K. M. Peng, High Pressure, Flux-Conserving Tokamak Equilibria, 0RNL/TM-5555, 0ak Ridge (August 1976). 
3. R. A. Dandl et a1., The E[MO Bumpy Torus Program, ORNL/TM-5451, Oak Ridge (Apri1 1976).

4. C. L. Hedrick et al., "Transport and Scaling in the E[MO Bumpy Torus (EBT)," Paper CN-35/D7, 6th Int. Conf. on Plasma Phys, and Cont. Nucl. Fus. Res., Berchtesgaden, Germany, FRG, Oct. 6-13, 1976.

5. G. E. Guest, C. L. Hedrick, and D. B. Nelson, Phys. Fluids $\underline{18}, 871$ (1975).

6. R. A. Dandl et al., Research Program for Plasma Confinement and Heating in ELMO Bumpy Torus Devices, ORNL/TM-4941, 0ak Ridge (June 1975).

7. W. W. Engle, Jr., "A User's Manual for ANISN, a One-Dimensional Discrete Ordinates Transport Code With Anisotropic Scattering," Report K-1693, Computing Technology Center, Union Carbide Corp. (1967).

8. M. Roberts and E. S. Bettis, Oak Ridge Tokamak Experimental Power Reactor Study Reference Design, ORNL/TM-5042, Oak Ridge (1975). 
APPENDIXES 
APPENDIX A

PLASMA ENGINEERING

\section{INTRODUCTION}

Plasma engineeringinvolves the use of the results from applied physics analyses in the design of fusion systems. Design process of the ELMO Bumpy Torus Reactor is difficult because some of the most critical aspects of the system and its behavior are not well understood at present; thus, the procedure involves making plausible estimates and retaining a flexibility so that the design can always accommodate new results from theoretical and experimental programs. This approach generates design criteria which will suggest the appropriate direction for engineering and technology development.

In this appendix the fundamental plasma and machine characteristics, system economics, and technology considerations are addressed. A commercial reactor is considered in which the design thermal power output is taken to be in the range of 2000 to $5000 \mathrm{MW}$. This is an important point. It may be possible to construct smaller reactors which are based on the EBT containment concept and which operate at lower design power levels; there is utility interest in such systems. Here the power output is taken to be comparable to typical low beta commercial reactor designs to permit comparisons between the two systems.

In a similar regard, the neutron wall loading has been restrained to values near $1 \mathrm{MW} / \mathrm{m}^{2}$. There is an incentive to increase the power density in a fusion reactor which in turn means that an increase in neutron wall loading must be tolerated. A modest increase in wall loading improves system economics but loadings above 3 or $4 \mathrm{MW} / \mathrm{m}^{2}$ will almost certainly be uneconomical due to reliability and pumping penalties. Recent material developments suggest that an increase in wall loading may be achievable. For the present EBT reference reactor, EBTR-48, the design wall loading will be taken to be near the conventional value of $1 \mathrm{MW} / \mathrm{m}^{2}$. In EBTR-48 the wall loading constraint limits the operating power density, which in turn results in $\beta \sim .25$. EBT physics analyses suggest that $\beta \sim .40$ to .50 may be tolerable. Emphasis to date has been focused on EBTR-48 although alternative, smaller-size reactors (EBTR-24) with increased power density, neutron wall loading, and beta are presently being studied. Some of the early results from the study are included here.

Finally, the plasma size is determined by specifying power output, power density, and neutron wall loading. Preliminary plasma dynamics simulations, equilibrium, and drift orbit considerations show that a plasma radius of about 1 meter is adequate to attain ignition assuming neoclassical scaling. However, a detailed physics basis fur the size required for the reactor plasma remains to be developed. Physics investigations under way are outlined in Appendix B. As in other system studies, system size and plasma parameters are determined self-consistently from models which show that they are adequate from a containment point of view. The ultimate requirements will be determined by experiments which will either validate the present assumptions or will provide the basis for change.

In the remainder of this appendix, the constraints and trade-offs which were used to determine the characteristics of the refcrence design are described.

\section{SYSTEM CIIARACTERISTICS}

\subsection{Neutron Wall Loading}

The first material boundary surrounding the plasma is assumed to be capable of sustaining a neutron wall loading, $L_{w}$, which is on the order of 1 to $3 \mathrm{MW} / \mathrm{m}^{2}$. This boundary exists at a distance equal to 
the average plasma radius, $a$, plus the cold zone, $\delta$, from the plasma center. Since each fusion event produces $17.6 \mathrm{MeV}$ of thermal energy, the total fusion thermal output, $P_{t h}$, produced in the plasma is

$$
\text { where } \quad \begin{aligned}
P_{\text {th }} & =L_{W} \times \frac{17.6}{14.1} \times A_{W}=L_{W} \frac{Q_{F}}{Q_{N}} A_{W} \\
& =2 \pi R_{0} 2 \pi(a+\delta), \\
& =(2 \pi)^{2} A^{2}(1+\delta / 0), \text { and } Q_{F}=17.6 \mathrm{McV}, Q_{N}=11.1 \mathrm{MeV} \\
\text { Finally, } \quad P_{\text {th }} & =L_{W} \frac{Q_{F}}{Q_{N}}(2 \pi)^{2} \mathrm{Aa}^{2}(1+\delta / a) .
\end{aligned}
$$

Note that in later discussions the total thermal output will be redefined to include blanket multiplications and an equivalent energy per fusion of $\sim 21 \mathrm{MeV}$.

Figure A-1 shows $P_{t h} / L_{w}$ for several values of aspect ratio, $A$, as a function of average plasma radius. Tabular results äre showh in lable A-1. (Note thal 0 느 20 Lill ls assumed lu be lie "cold zone" in all cases.)

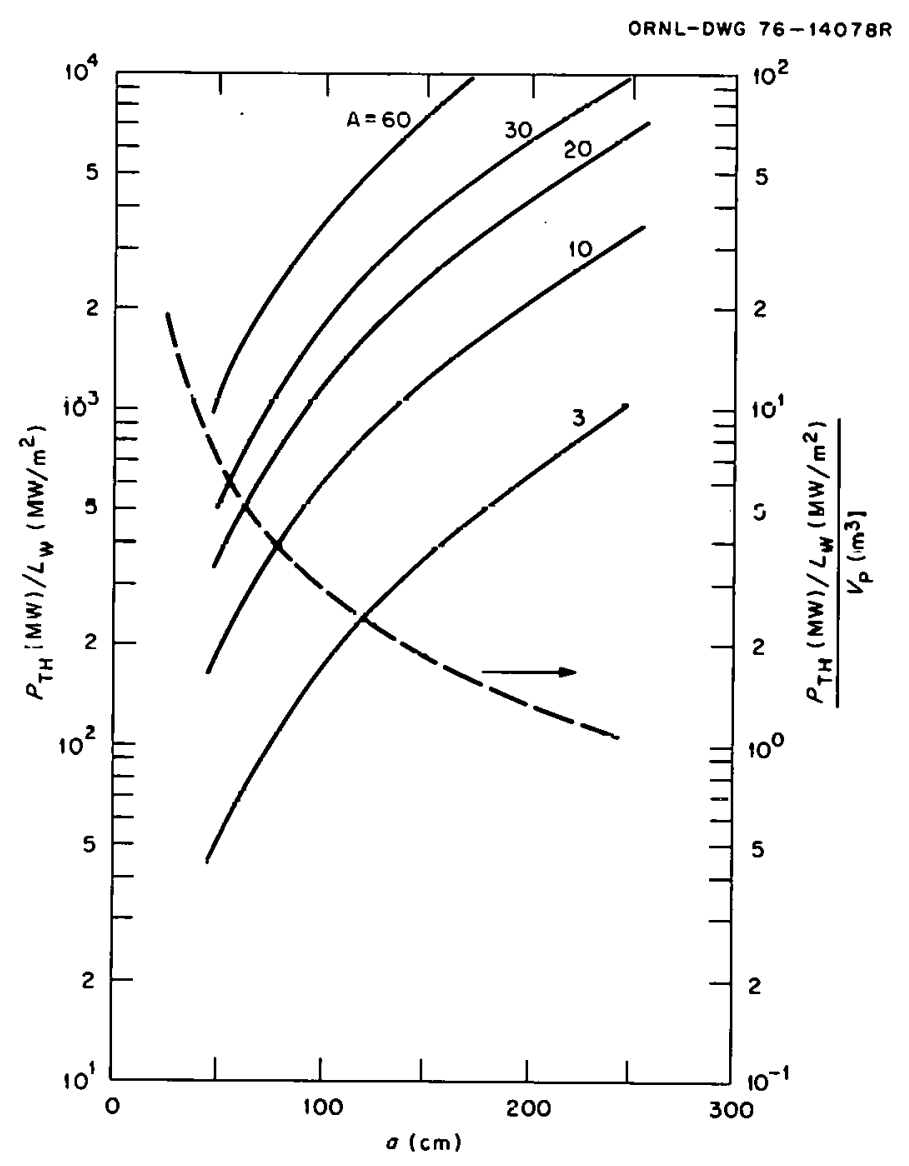

Fig. A-1. Relationship between genmetrical and power production parameters. 
TABLE $\cdot A-1$

Thermal Power and Wall Loading Characteristics

\begin{tabular}{cccccc}
$\begin{array}{c}P_{t h}(M W) / L_{W}\left(M W / m^{2}\right) \\
(\mathrm{cm})\end{array}$ & $\begin{array}{c}A=3 \\
(-)\end{array}$ & $\begin{array}{c}A=10 \\
(-)\end{array}$ & $\begin{array}{c}A=20 \\
(-)\end{array}$ & $\begin{array}{c}A=30 \\
(-)\end{array}$ & $\begin{array}{c}A=60 \\
(-)\end{array}$ \\
\hline 50 & 51.6 & 172 & 344 & 516 & 1032 \\
75 & 105 & 351 & 702 & 1053 & 2106 \\
100 & 177 & 591 & 1182 & 1773 & 3546 \\
125 & 268 & 893 & 1786 & 2679 & 5358 \\
150 & 377 & 1257 & 2514 & 3771 & 7542 \\
175 & 505 & 1682 & 3364 & 5046 & 10092 \\
200 & 650 & 2168 & 4336 & 6504 & 13008 \\
225 & 815 & 2716 & 5432 & 8148 & 16296 \\
250 & 998 & 3326 & 6652 & 9978 & 19956
\end{tabular}

\subsection{Plasma Thermal Power Density}

The wall loading is, of course, coupled to the thermal power density in the plasma. This power density in turn will dictate the desirable characteristics for the plasma. For example,

$$
\left(\frac{P_{t h}}{V_{P}}\right) \times V_{P}=L_{W} \times A_{W} \times\left(\frac{Q_{F}}{Q_{N}}\right),
$$

where $V_{p}$ is the plasma volume. Thus,

$$
\left[\frac{{ }^{t_{h}} / L_{w}}{V_{P}}\right]=\left(\frac{Q_{F}}{Q_{N}}\right) \times \frac{2}{a}(1+\delta / a) .
$$

Note that for an assumed wall loading, the thermal power density is independent of $A$ and depends only on $\delta$ and $a$. These results are shown graphically in Fig. A-1 and tablulated in Table A-2.

TABLE A-2

Thermal Power Density Characteristics

\begin{tabular}{lc}
$\mathrm{a}(\mathrm{cm})$ & $\frac{P_{t h}(M W) / L_{W}\left(M W / \mathrm{m}^{2}\right)}{V_{P}\left(\mathrm{~m}^{3}\right)}$ \\
\hline & \\
50 & 6.99 \\
75 & 4.22 \\
100 & 3.00 \\
125 & 2.32 \\
150 & 1.89 \\
175 & 1.59 \\
200 & 1.37 \\
225 & 1.21 \\
250 & 1.08 \\
\hline
\end{tabular}




\subsection{Plasma Parameters}

The plasma power density required to attain a particular wall loading in a given size device has been determined. This in turn dictates the range of plasma densities and temperatures required to produce the power. That is,

$$
\frac{P_{\text {th }}}{V_{P}}=\frac{N_{i}^{2}<\sigma v>Q_{F}}{4}
$$

where $N_{i} / 2=N_{0}=N_{T}$

$$
Q_{F}=17.6 \times 1.6 \times 10^{-19} \mathrm{MJ} \text {. }
$$

Figure A-2 shows the density/temperature characteristics which correspond to a particular power density. Table A-3 presents the same data in tabular form.

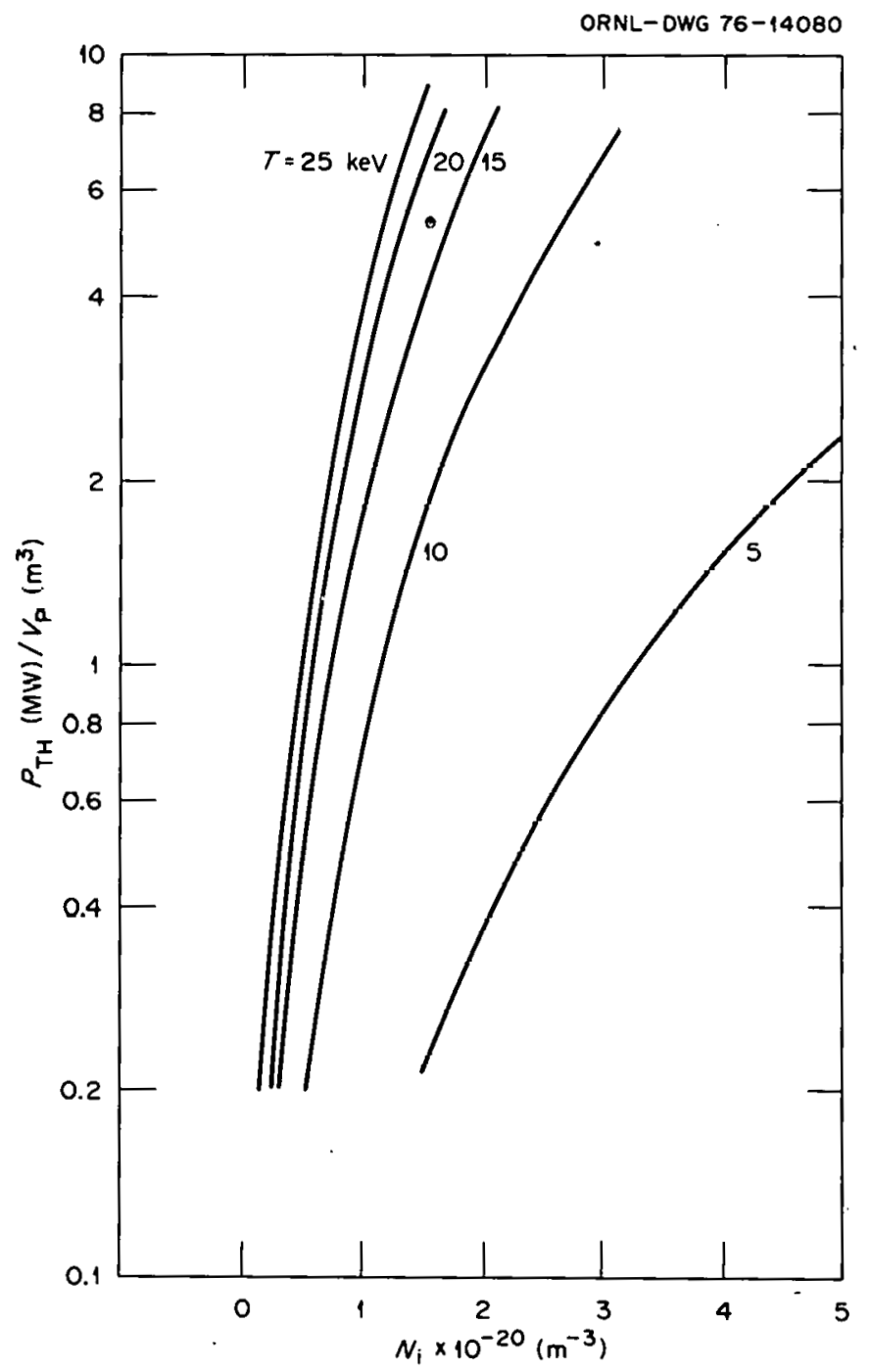

Fig. A-2. Relationship between density/temperature and power density. 
TABLE A-3

Density/Temperature Characteristics

$$
P_{t h}(M W) / v_{p}\left(m^{3}\right)
$$

\begin{tabular}{rcccc}
$\begin{array}{c}\mathrm{N}_{i} \\
\left(\mathrm{~m}^{-3}\right)\end{array}$ & $\begin{array}{c}\mathrm{T}=5 \mathrm{keV} \\
<\mathrm{v}>1.35 \times 10^{-23}\end{array}$ & $\begin{array}{c}10 \\
1.13 \times 10^{-22}\end{array}$ & $2.71 \times 10^{-22}$ & $5.63 \times 10^{-22}$ \\
\hline $1 \times 10^{19}$ & $9.51 \times 10^{-4}$ & $7.96 \times 10^{-3}$ & $1.91 \times 10^{-2}$ & $3.96 \times 10^{-2}$ \\
$1.5 \times 10^{19}$ & $2.14 \times 10^{-3}$ & $1.79 \times 10^{-2}$ & $4.29 \times 10^{-2}$ & $8.92 \times 10^{-2}$ \\
$2 \times 10^{19}$ & $3.80 \times 10^{-3}$ & $3.18 \times 10^{-2}$ & $7.63 \times 10^{-2}$ & $1.58 \times 10^{-1}$ \\
$5 \times 10^{19}$ & $2.38 \times 10^{-2}$ & $1.99 \times 10^{-1}$ & $4.77 \times 10^{-1}$ & $9.91 \times 10^{-1}$ \\
$1 \times 10^{20}$ & $9.51 \times 10^{-2}$ & $7.96 \times 10^{-1}$ & 1.91 & 3.96 \\
$1.5 \times 10^{20}$ & $2.14 \times 10^{-1}$ & 1.79 & 4.29 & 8.92 \\
$2 \times 10^{20}$ & $3.80 \times 10^{-1}$ & 3.18 & 7.63 & $1.58 \times 10^{1}$ \\
$5 \times 10^{20}$ & 2.38 & $1.99 \times 10^{1}$ & $4.77 \times 10^{1}$ & $9.91 \times 10^{1}$
\end{tabular}

The power density can also be written in terms of $\beta$ (ratio of plasma pressure to magnetic energy density) and $B$, magnetic field strength. Then,

$$
\frac{P_{t h}}{V_{P}}=\frac{\beta^{2} B^{4}}{(4 \mu)^{2}} \frac{\text { LaV }}{4 T^{2}} Q_{F}
$$

A unique value of $B^{2} B^{4}$ exists when values for the temperature and power density are assumed. Table A-4 shows the corresponding parameters in this regard.

TABLE A-4

\begin{tabular}{|c|c|c|c|c|}
\hline \multirow[b]{2}{*}{$B^{2} B^{4}$} & \multicolumn{4}{|c|}{$P_{t h}(M W) / v_{p}\left(m^{3}\right)$} \\
\hline & $\begin{aligned} T & =5 \mathrm{keV} \\
\langle\sigma \mathrm{V}\rangle & =1.35 \times 10^{-22}\end{aligned}$ & $\begin{array}{c}10 \\
1.13 \times 10^{-22} \\
\end{array}$ & $\begin{array}{l}15 \\
2.71 \times 10^{-22} \\
\end{array}$ & $5.63 \times 10^{-22}$ \\
\hline 0.015 & $5.877 \times 10^{-3}$ & $1.23 \times 10^{-2}$ & $1.31 \times 10^{-2}$ & $9.80 \times 10^{-22}$ \\
\hline 0.015 & $8.82 \times 10^{-3}$ & $1.845 \times 10^{-2}$ & $1.967 \times 10^{-2}$ & $1.47 \times 10^{-2}$ \\
\hline 0.02 & $1.175 \times 10^{-2}$ & $2.46 \times 10^{-2}$ & $2.625 \times 10^{-2}$ & $1.96 \times 10^{-2}$ \\
\hline 0.05 & $2.938 \times 10^{-2}$ & $6.15 \times 10^{-2}$ & $6.55 \times 10^{-2}$ & $4.90 \times 10^{-2}$ \\
\hline 0.1 & $5.877 \times 10^{-2}$ & $1.23 \times 10^{-1}$ & $1.31 \times 10^{-1}$ & $9.80 \times 10^{-2}$ \\
\hline 0.15 & $8.82 \times 10^{-2}$ & $1.845 \times 10^{-1}$ & $1.96 / \times 10^{-1}$ & $1.47 \times 10^{-1}$ \\
\hline 0.2 & $1.175 \times 10^{-1}$ & $2.46 \times 10^{-1}$ & $2.625 \times 10^{-1}$ & $1.96 \times 10^{-1}$ \\
\hline 0.5 & $2.938 \times 10^{-1}$ & $6.15 \times 10^{-1}$ & $6.55 \times 10^{-1}$ & $4.90 \times 10^{-1}$ \\
\hline 1.0 & $5.877 \times 10^{-1}$ & 1.23 & 1.31 & $9.80 \times 10^{-1}$ \\
\hline 1.5 & $8.82 \times 10^{=1}$ & 1.845 & 1.967 & 1.47 \\
\hline 2.0 & 1.175 & 2.46 & 2.625 & 1.96 \\
\hline 5.0 & 2.938 & 6.15 & 6.55 & 4.90 \\
\hline 10.0 & 5.877 & $1.2 .3 \times 10^{1}$ & $1.31 \times 10^{1}$ & 9.80 \\
\hline
\end{tabular}

Beta-Magnetic Field Characteristics 
Finally, the trade-offs between $B$ and $B$ must be considered. High-beta, low- $B$ operation is desirable. However, the minimum field strength assumed feasible is $\sim 25 \mathrm{kG}$. This is consistent with the cold zone thickness of $20 \mathrm{~cm}$, i.e., two gyroradii of a 3.5-MeV alpha particle in a 25-kG field, and with the assumption that confinement time will increase with an increase in magnetic field. $B \sim 25 \mathrm{kG}$ is taken to be a practical minimum in this work. Table A-5 shows the B-B data. Figure A-3 shows the same data graphically.

TABLE A-5

$\underline{B-B \text { Trade-0ffs }}$

$B(T)$

\begin{tabular}{llllll}
$B^{2} B^{4}$ & $B=0.05$ & 0.1 & 0.2 & 0.3 & 0.4 \\
\hline 0.01 & 1.41 & 1.0 & $7.05 \times 10^{-1}$ & $5.76 \times 10^{-1}$ & $5.0 \times 10^{-1}$ \\
0.015 & 1.66 & 1.11 & $7.80 \times 10^{-1}$ & $0.37 \times 10^{-1}$ & $5.55 \times 10^{-1}$ \\
0.02 & 1.68 & 1.19 & $8.40 \times 10^{-1}$ & $6.86 \times 10^{-1}$ & $3.95 \times 10^{-1}$ \\
0.05 & 2.11 & 1.50 & 1.06 & $8.61 \times 10^{-1}$ & $7.5 \times 10^{-1}$ \\
0.1 & 2.51 & 1.77 & 1.25 & 1.02 & $8.85 \times 10^{-1}$ \\
0.15 & 2.78 & 1.96 & 1.39 & 1.13 & $9.80 \times 10^{-1}$ \\
0.2 & 2.99 & 2.11 & 1.50 & 1.22 & 1.06 \\
0.5 & 3.76 & 2.66 & 1.88 & 1.54 & 1.33 \\
1.0 & 4.47 & 3.16 & 2.24 & 1.82 & 1.58 \\
1.5 & 4.95 & 3.50 & 2.48 & 2.02 & 1.75 \\
2.0 & 5.32 & 3.76 & 2.66 & 2.17 & 1.88 \\
5.0 & 6.69 & 4.73 & 3.34 & 2.73 & 2.36 \\
10.0 & 7.95 & 5.62 & 3.98 & 3.24 & 2.81
\end{tabular}

\subsection{Microwave Frequencies}

In addition to the magnetic and plasma physics constraints described above, the required microwave frequencies must be considered. A magnetic field of $\sim 40-45 \mathrm{kG}$ exists near the magnet throat. The resonant frequency there is $\sim 120 \mathrm{GHz}$. This frequency is above that required by cutoff ( $\left.\omega_{\text {pe }}\right)$ and is suitable for background plasma heating. Due to the decreased density and field in the region of the stabilizing annuli, microwave frequencies less than..this by a factor of 2 may be acceptable for sustaining the annuli. A decision on the means of heating the EBTR plasma and sustaining its annuli has not been made but the frequency requirements, if microwaves are used, are compatible with those presently under development, i.e., $120 \mathrm{GHz}$. This includes the case where $f_{\mu}($ annulus $)=? \times(\omega \mathrm{ce} / 2 \pi)$.

Since microwave heating is anticipated in the machine, there are two main frequencies of interest. First, the electron cyclotron frequency:

$$
f_{c e}=\omega_{c e} / 2 \pi=\frac{1}{2 \pi} \times \frac{e b}{m_{e}},
$$

and, second, the plasma frequency:

$$
f_{p e}=\omega_{p e} / 2 \pi-\frac{1}{2 \pi}\left[\frac{4 \pi N_{e} e^{2}}{m_{e}}\right]^{\frac{1}{2}} .
$$

In the present design neutral beam heating for the background plasma is assumed. It is also assumed that the stabilizing annuli will be sustained by microwaves. Detailed magnetic field contours and electron density profiles are necessary to permit a determination of the precise microwave frequency required in the reactor. Table A- 6 shows the resonant field and electron density which result 
in cutoff for a given frequency. A frequency of $120 \mathrm{GHz}$ is resonant at $\sim 43 \mathrm{kG}$ and can penetrate densities $\sim 1.8 \times 10^{20} / \mathrm{m}^{3}$. This frequency is considered to be an upper 1 imit for the reactor application.

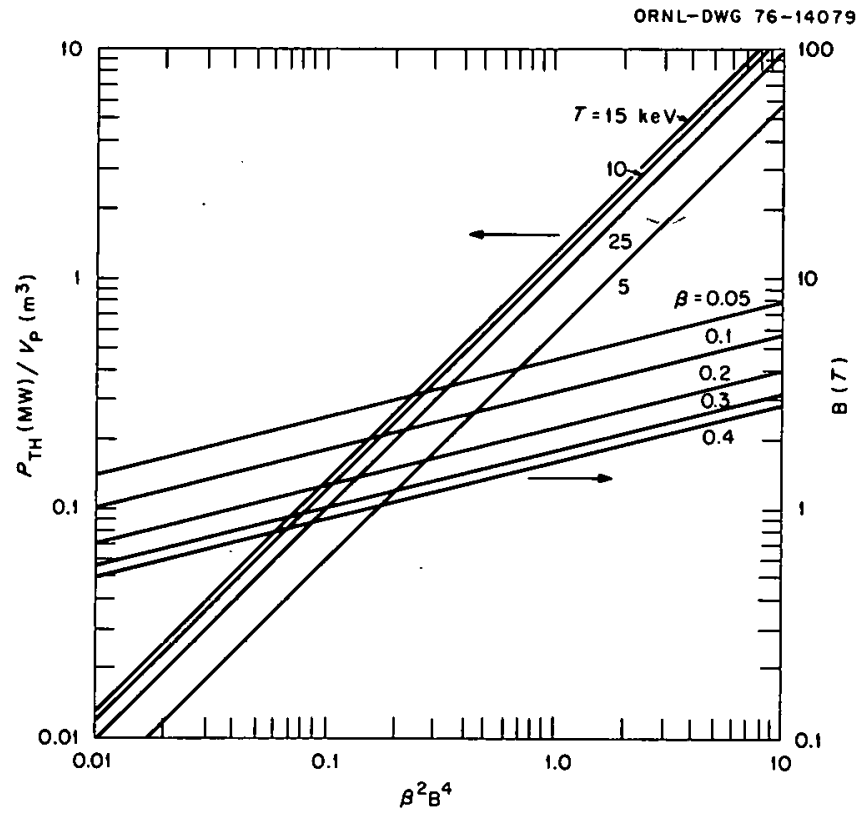

Fig. A-3. Relationship between plasma, magnetic field, and power production parameters.

TABLE A-6

Resonant Fields and Cutoff Densities vs. Frequency

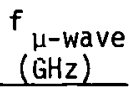

(GHz)

50

60

70

80

90

100

110

120
B

(T)

1.78

2.14

2.50

2.86

3.22

3.57

3.93

4.29

\section{$\mathrm{Ne}$ $\left(\# / m^{3}\right)$}

$3.1 \times 10^{19}$

$4.45 \times 10^{19}$

$6.0 \times 10^{19}$

$7.94 \times 10^{19}$

$1.0 \times 10^{20}$

$1.24 \times 10^{20}$

$1.5 \times 10^{20}$

$1.78 \times 10^{20}$ 


\subsection{Mirror Ratio and Magnet Spacing}

A choice of magnetic field strength can be made at this point. The final information needed to specify the basic parameters for the system depends on the magnetic field coils. In the high aspect ratio approximation the following equation relates the field strength at various radii and toroidal locations to the number of coils, the current, the coil radius, and the spacing between coils:

$$
B(r, \phi) \simeq \frac{2 N I}{R}\left\{1+\frac{2 N d}{R} \sum_{n=1} n K_{1}\left(n \frac{N d}{R}\right) I_{0}\left(n \frac{N r}{R}\right) \operatorname{Cos}(n N \phi)\right\}
$$

R: Major radius

I: Current (per coil)

$\mathrm{N}$ : Number of toroidal sectors

d: Mirror radius (coil radius at the current center)

s: Co11 spacing $(2 \pi R / N)$

$I_{0}$ and $K_{1}$ : Modified Bossol functions

For a specific mirror ratio (the maximum field at the coll throat compared to the field on the midplane), this equation can be rewritten as follows:

$$
M \simeq \frac{1+\frac{2 N d}{R} K_{1}\left(\frac{N d}{R}\right)}{1-\frac{2 N d}{R} K_{1}\left(\frac{N d}{R}\right)}
$$

For example, by choosing a mirror ratio of about 2 , a blanket and shield thickness, $t_{\text {bs, }}$ a cold zone, $\delta$, and a radial thickness for the coil, $t_{c}$, the number of coils required can be approximated by

$$
N \simeq \frac{\text { Aa } \times 2.62}{a+\delta+t_{\text {Ls }}+t_{L} / 2}
$$

(Note that $A=60, a=1 \mathrm{~m}, \delta=0.2 \mathrm{~m}, t_{b s}=1.75 \mathrm{~m}$ and $t_{g} / 2=0.35 \mathrm{~m}$ yields $\mathrm{N} \nexists 48 \mathrm{coils}$. )

\subsection{Reference Parameters}

The information developed in the previous paragraphs was used to establish the parameters for the self-consistent EBT reactor reference design. The results are summarized in Table A-7.

It should be noted that a large aspect ratio torus is implicit in the above set of requirements; it is dictated by the simultaneous constraints imposed on coil spacing and mirror ratio. lhe mirror ratio required for the formation of a high-beta mirror plasma fixes the mirror curvature. The coil radius and mirror ratio determination fixes the intercoil spacing within narrow 1 imits. The aspect ratio then has a lower bound which must be such that the toroidal curvature is smaller than that fixed by equilibrium considerations, i.e., mirror curvature. From an economic point of view, it is desirable to optimize the system parameters. Preliminary work has begun in this area but emphasis to date has not been on this optimization procedure. 
TABLE A-7

EBT Reactor Reference Parameters

\begin{tabular}{|c|c|c|c|}
\hline & $\underline{E B T R-48^{1}}$ & \multicolumn{2}{|c|}{ EBTR-24 2} \\
\hline Plasma radius, $a(m)$ & 1.0 & \multicolumn{2}{|r|}{1.0} \\
\hline Aspect ratio, $A(-)$ & 60 & \multicolumn{2}{|r|}{30} \\
\hline Major radius, $R_{0}(m)$ & 60 & \multicolumn{2}{|r|}{30} \\
\hline Mirror ratio, $M(-)$ & 1.78 & \multicolumn{2}{|c|}{1.78} \\
\hline Ion temperature, $T_{i}(\mathrm{keV})$ & 15 & \multicolumn{2}{|r|}{15} \\
\hline Ion density, $N_{j} \times 10^{-20}$ & 1.2 & 1.25 & 2.13 \\
\hline Beta, $B(\%)$ & 25 & 24 & 42 \\
\hline Magnetic field, $B_{T}$ (on axis) $(T)$ & $2.5-4.5$ & 2.5 & -4.5 \\
\hline Number of coils, $\mathrm{N}_{\mathrm{c}}(-)$ & 48 & \multicolumn{2}{|r|}{24} \\
\hline Total fusion power, $\mathrm{P}(\mathrm{MW})$ & 4000 & 1775 & 5300 \\
\hline Power density, $P / V_{p}\left(M W / \mathrm{m}^{3}\right)$ & 3.37 & 3.0 & 9.0 \\
\hline Neutron wall loading, $L_{W}\left(M W / m^{2}\right)$ & 1.13 & 1 & 3 \\
\hline Cold zone, $\delta(m)$ & 0.2 & \multicolumn{2}{|c|}{0.2} \\
\hline Blanket and shield thickness, $t_{s b}(m)$ & 1.75 & \multicolumn{2}{|c|}{1.75} \\
\hline Coil inner radius, $r_{c}(m)$ & 2.95 & \multicolumn{2}{|c|}{2.95} \\
\hline Current density, $\mathrm{J}_{C}\left(\mathrm{~A} / \mathrm{cm}^{2}\right)$ & 1500 & \multicolumn{2}{|c|}{1500} \\
\hline Coil radial thickness, $t_{c}(m)$ & 0.71 & \multicolumn{2}{|c|}{0.71} \\
\hline Coil half length, $L / 2(\mathrm{~m})$ & 1.30 & \multicolumn{2}{|c|}{1.30} \\
\hline
\end{tabular}

${ }^{1}$ Emphas is to date has been focused. on EBTR-48.

${ }^{2}$ Alternative smaller size reactors with increased neutron wall loading are presently under study.

\section{SYSTEM ECONOMICS}

Even at this early stage of the study, several conclusions can be drawn from a preliminary economic analys is of an EBT reactor. The absolute values of reactor component costs are not as important as the relationships between the costs; the fact that these relationships can be quantified for certain assumptions allows some design and operation constraints to. be specified.

$\Lambda$ simple energy balance which accounts for the circulating power in the system defines a figure of merit for the reactor. An upper limit for the recirculating power fraction (defined as the recirculating power divided by the power produced) is specified by a cost breakdown of the reactor, using limiting assumptions for the cost of reactor components and of the recirculating power. Some of the recirculating power is used for auxiliary functions such as magnet refrigeration and blanket cooling, while the rest is available to be fed back into the plasma. Since the auxiliary power requirements can be relatively well defined, the upper limit on the recirculating power fraction effectively restricts the amount of power available for feedback into the plasma, and hence demands that certain plasma conditions be fulfilled.

The economic analysis strongly indicates that an EBT reactor should be an ignition device and specifies limits on the feedback power avaijable to sustain the plasma conditions. In the simplest case the sustenance power is considered to be that necessary to drive the stabilizing annuli. In high wall loading models, the coolant pumping power must also be taken into account. The feedback power 
limitation implies that almost all of the plasma heating is due to the thermonuclear alpha particles. This, in turn, specifies an energy containment time that must be attained by the plasma system.

The effect on reactor capital cost of the physical dimensions of the reactor is also investigated. The approach is based on trade-off analyses in order to determine to what extent a reduction in reactor size reduces the cost of electricity. The total cost of the reactor is assumed to be made up of two components - the nuclear island (consisting of blanket structure and magnet costs), and the rest of the reactor. For a fixed power output, a change in reactor size directly affects the nuclear island cost while the rest of the costs stay essentially constant. By further breaking up the cost of the nuclear island into its components, the sensitivity of reactor capital cost to reactor size is investigated. The main conclusion is that a smaller reactor costs proportionally less only under certain restrictive conditions. In any case, the savings in cost must be balanced against the resulting increase in structural complexity and the possible detrimental effects on the plasma which may result from increased curvature.

\subsection{Power Balance and Economic Constraints}

\subsubsection{Recirculating power fraction and conversion efficiency}

Consider the simple reactor power balance shown in Fig. A-4. The thermal energy, $P_{\text {th }}$, includes both the fusion power produced and the power produced by blanket multiplication. $P_{\text {th }}$ is converted to electricity with a net conversion efficiency $\eta_{T}$. Assuming that the system requires an input maintenance power, $P_{M}$, then the power available to the grid is

$$
P_{g}=P_{e}-P_{M}
$$

or

$$
Q=P_{q} / P_{M}=Q^{*}-1
$$

where

$$
Q^{\star}=P_{e} / \mu_{M}
$$

and $\mathrm{P}_{\mathrm{e}}$ is the total electric power produced. The quanificy $\mathrm{Q}^{\star}$ is a figure of mertt fur the reactur. A larger value of $Q^{\star}$ implies a lower recirculating power fraction and a higher overall reactor efficiency.

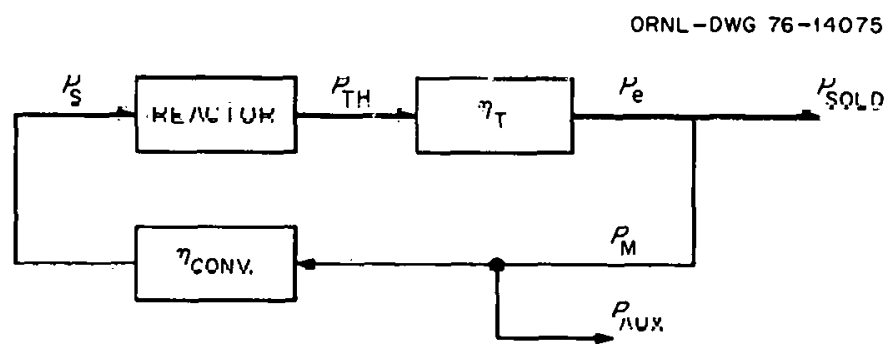

Fig. A-4. Reactor power balance. 
The quantities $P_{e}$ and $P_{M}$ can be expressed in terms of better defined reactor parameters. For example, for a given reactor size and wall loading, $P_{t h}$, the gross thermal power is fixed. Since

$$
P_{e}=\eta_{T} P_{\text {th }} \text {, }
$$

where $n_{T}$ is the overall thermal-electric conversion efficiency, specifying $n_{T}$ fixes $P_{e}$.

Assuming a steady state reactor operating with a divertor system, and defining two conversion efficiencies - $n_{b}$ (for the conversion of the thermal energy deposited in the blanket) and $n_{0}$ (for the conversion of the thermal energy deposited in the divertor or first wall) - yields

$$
P_{e}=n_{b} p_{n}(1+m)+n_{D}\left(p_{\alpha}+p_{s}\right)
$$

where $P_{n}$ is the 14-MeV neutron power, $m$ is the blanket multiplication factor per neutron, and $P_{S}$ is the power injected into the plasma. $P_{s}$ includes the power that is delivered to the plasma to sustain the stabilizing annuli. The total thermal power is

$$
P_{\text {th }}=P_{n}(1+m)+P_{\alpha}+P_{s} \text {. }
$$

Combining Eqs. $(4,5$, and 6$)$ yietds

$$
n_{T}=\frac{n_{b}\left[(1+m) \frac{P_{n}}{P_{T}}+\frac{n_{D}}{\eta_{b}}\left(\frac{P_{\alpha}}{P_{T}}+\frac{P_{s}}{P_{T}}\right)\right]}{(1+\cdots) \frac{P_{n}}{P_{T}}+\frac{P_{\alpha}}{P_{T}}+\frac{P_{s}}{P_{T}}},
$$

where $P_{T}$ is the total energy produced/fusion event. For small values of $P_{s} / P_{n}\left(Q^{*}>1\right)$, and assuming that $21 \mathrm{MeV}$ is released per fusion event, (i.e., the blanket multiplication per neutron is 1.24 ),

$$
n_{T}=0.83 n_{b}+0.17 n_{D}
$$

If $n_{b}=n_{D}$ then $n_{T}=n_{b}$; if $n_{D}=0$, then $n_{T}=0.83 n_{b}$. Further, if $n_{\text {conv }}$ is the efficiency of injection, i.e., efficiency of converting electrical power into power absorbed by the plasma,

$$
P_{M}=\frac{P_{S}}{\ddot{\eta}_{\text {conv }}}+P_{\text {aux }} \text {, }
$$

where $P_{\text {aux }}$ is the power required for refrigeration, pumping, and other auxiliary loads. Thus, $Q^{*}$ may be expressed as:

$$
Q^{*}=\frac{\eta_{b}(1+m) P_{n}+n_{D}\left(p_{\alpha}+p_{s}\right)}{\frac{P_{s}}{n_{\text {conv }}}+P_{\text {aux }}} \text {. }
$$

\subsubsection{Reactor cost dependence on $Q^{\star}$}

The impact of not having an ignition device is considered in this section. In particular, the limiting value of $Q^{\star}$ from an economic point of view is determined. It should be realized that the cost figures presented here are expected values rather than exact ones and that the conclusions pertain to the variation in costs rather than to the absolute costs themselves. In order to allow for cost variations a range of values is chosen for each component cost. 
The system is divided into five cost groups ${ }^{1}$ :

1. Liquid metal primary coolant system $\left(C_{L i}\right)$. This is defined as the cooling system for the blanket and divertor including piping and pumps.

2. The energy conversion (steam) system $\left(\mathrm{C}_{\mathrm{st}}\right)$. Cost assumptions are made for the steam turbines, condensers, heat exchangers, and cooling towers.

3. Section and divertor costs $\left(C_{s e c}\right)$. A section is defined as the basic mirror segment which is repeated to form the EBT reactor. Material and construction costs for the reactor blanket, divertors, and magnets are included.

4. The auxiliary costs of the plant $\left(c_{\text {aux }}\right)$. These include land, structures, site facilities, auxiliary reactor plant, and other fixed costs of the system.

5. Sustenance power cost $\left(C_{s}\right)$. The cost of the conversion system required to convert electrical power into power that can be delivered to and absorbed by the plasma. In the case of the particular reactor investigated, this would be the cost of the microwave system. The power required by auxiliary reactor functions is neglected. Table A- 8 shows the range of values investigated.

TABLE A-8

Range of Costs (in arbitrary units)

\begin{tabular}{|c|c|c|c|}
\hline \multirow[b]{2}{*}{ Primary coolant } & \multicolumn{2}{|c|}{$\cos t / k W_{e}$} & \multirow{2}{*}{$\frac{\text { Scale Factor }}{0.8}$} \\
\hline & 100 & 200 & \\
\hline Steạm plant & & & 1 \\
\hline Section cost & 300 & 500 & 1 \\
\hline Auxiliary cost & & & 0.75 \\
\hline Sustenance power & 10 & 200 & 1. \\
\hline
\end{tabular}

The scale factor, e, in Table A-8 takes into account the effect of size scaling:

$$
\frac{\operatorname{Cost} 2}{\operatorname{size} 2}=\frac{\operatorname{Cost} 1}{\operatorname{size} 1}\left[\frac{\operatorname{Size} 2}{\operatorname{size} 1}\right]^{e-1} \text {. }
$$

The value of 0.8 and 0.75 for primary coolant and auxiliary cost scaling are taken from Ref. 1 . The size scaling factor of unity for the section/divertor cost is justified by the argument that the sector size is relatively invariant for two reactors with the same mirror ratio and the same neutron wall loading. The cost/kW of the nuclear island is relatively constant between two such cases. The cost per $\mathrm{kW}_{\mathrm{e}}$ available to the grid may be expressed as follows:

$$
C=\frac{C_{L i}(R)^{-2}+C_{s t}+C_{s e c}+C_{\text {aux }}(R)^{-.25}, C_{S}}{1-\frac{1}{Q^{\star}}},
$$

where $R$ is given by

$$
R=\frac{P_{e}}{1905} .
$$

The results are shown in Fig. A-5. Even for widely varying sustenance power costs and for varying reactor sizes, the curves show that for small $Q^{*}$ the cost/kW rises sharply. This is not surprising, since a small $Q^{\star}$ implies less power sold for essentially the same capital cost. At 
higher values of $Q^{*}$ the unit power cost decreases and is relatively flat for $Q^{*}>20$. The absolute value assumed for the unit cost of sustenance power does not influence significantly the cost/kW at high $Q^{\star}$. These curves show quite dramatically that for values of $Q^{\star}$ less than $\sim 8-10$, economic considerations indicate that the EBT reactor becomes less attractive. For a $1000 \mathrm{MW}_{\mathrm{e}} \mathrm{plant}$, this implies that the amount of electric power fed back into the plant must be less than $125 \mathrm{MW}_{\mathrm{e}}$.
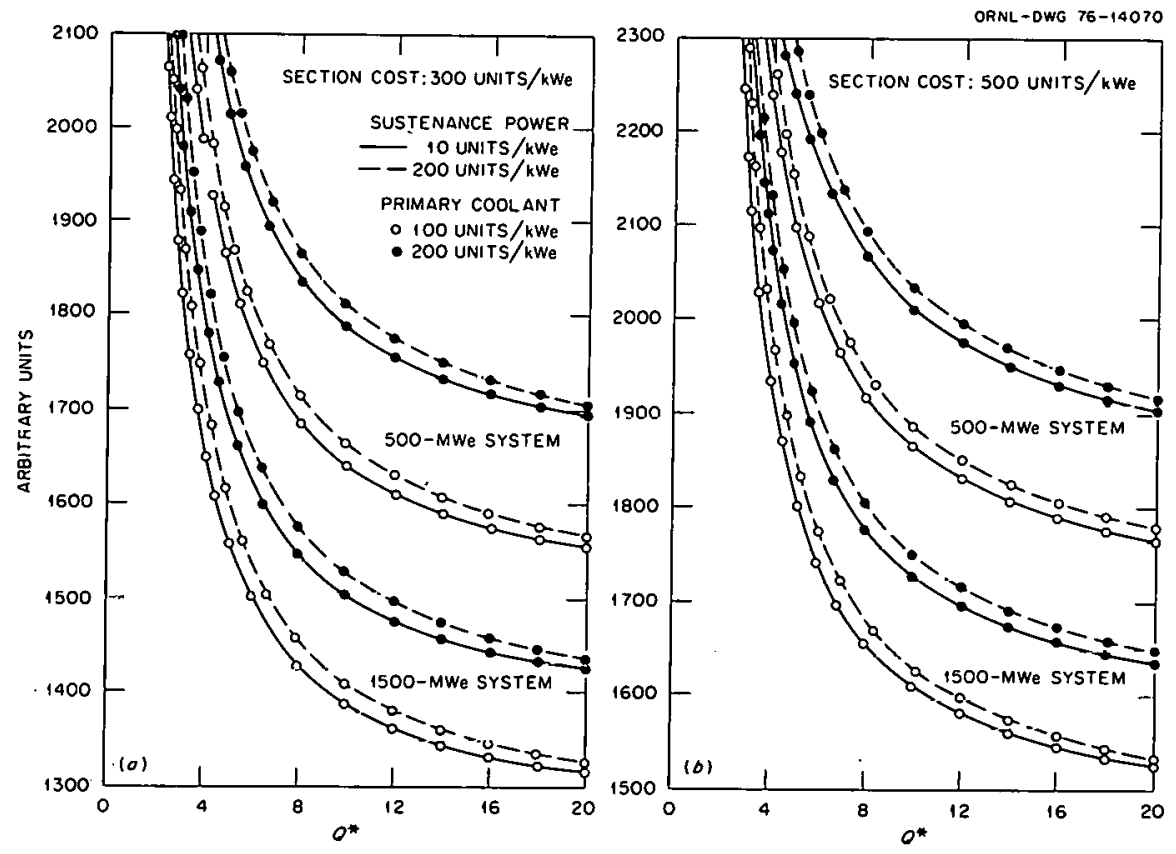

Fig. A-5. Cost/kWe (sold) versus $Q^{\star}$

\subsubsection{Implications of a $Q^{*}$ limit}

The maintenance power discussed in the previous sectiun is made up uf two compinents. From Eq. (9) it. can be shown that

$$
\frac{P_{\text {aux }}}{P_{e}}=\frac{1}{Q^{*}}-\frac{P_{s}}{\eta_{\text {conv }} P_{e}} .
$$

Figure A-6 shows the dependence of $P_{\text {aux }} / P_{e}$ on $P_{s} / P_{e}$ for various values of $Q^{*}$ and $n_{\text {conv }}$ Basically, this plot can be used to determine whether or not a particular reactor satisfies the tmposed economic constraints. For example, if the power required for refrigeration and cooling is $\sim 10 \mathrm{MW}_{\mathrm{e}}$, the microwave power required to sustain the stabilizing annuli in a $1000 \mathrm{MW}$ reactor must be less than $92 \mathrm{MW}_{\mathrm{e}}$. This assumes that the electric-to-microwave conversion efficiency is $80 \%$. The limitation is $52 \mathrm{MW}$ for a conversion efficiency of $45 \%$. If the refrigeration and cooling power requirements increase, the power available to sustain the plasma decreases correspondingly. 


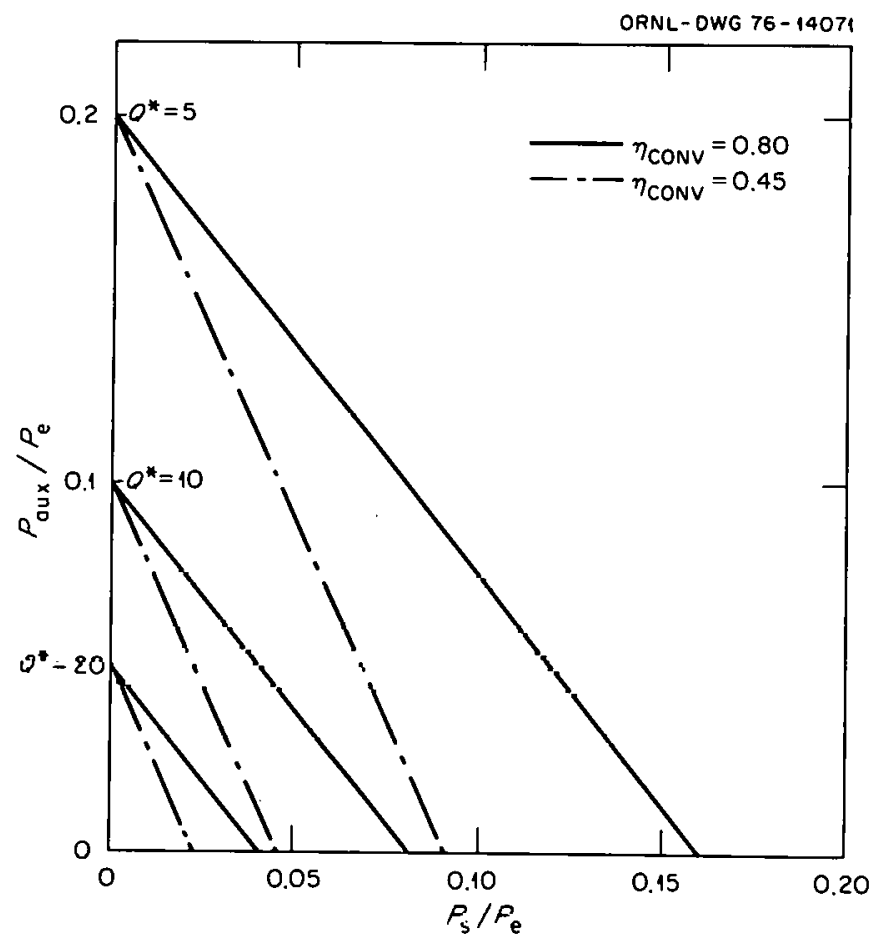

Fig. A-6. Allowable sustenance power for various $Q^{\star}$.

One final piece of information can be extracted from the limiting value of Q*. Note that in steady state

$$
\xi P_{i L}+P_{s}=\frac{W_{p}}{\tau_{L}},
$$

where $\xi$ is the fraction of the alpha energy produced which is deposited in the plasma, $W_{p}$ is the stored plasma energy and $\tau_{L}$ is the global energy confinement time for the plasma. Defining $\tau^{\star}$ as the stored plasma energy divided by the total fusion power produced, or

$$
\tau *=\frac{W_{p}}{P_{T}},
$$

and substituting into Eq. (15),

$$
\frac{P_{S}}{P_{n}}=\frac{P_{T}}{P_{n}} \cdot \frac{\tau^{\star}}{\tau_{L}}-\xi\left(\frac{P_{\alpha}}{P_{n}}\right) .
$$

Finally, substituting this result into Eq. (10) and assuming $n_{b}=n_{D}$, yields

$$
Q^{*}=\frac{n_{t}{ }^{\prime \prime} \operatorname{conv}\left[1+m+(1-\xi) \frac{P_{\alpha}}{P_{n}}+\frac{P_{T}}{P_{n}} \frac{\tau^{*}}{\tau_{L}}\right]}{\frac{P_{T}}{P_{n}} \frac{\tau^{*}}{\tau_{L}}-\xi \frac{\alpha}{P_{n}}+\frac{P_{\text {aux }}}{P_{n}} \cdot n_{\text {conv }}} \text {. }
$$


Figure $A-7$ shows $Q^{*}$ as a function of the normalized confinement time for two values of $\xi$. Note that $\tau^{\star}$ is the confinement time required for breakeven, i.e., the confinement time which would permit breakeven if the total energy produced could be delivered back into the plasma with an efficiency of $100 \%$.

Figure A-7 shows two values of $\eta_{\text {conv }}$ and two values of the ratio $P_{\text {aux }} / P_{n}$. A value of 0.005 for $P_{\text {aux }} / P_{n}$ (Figs. A-7b and A-7c) implies that for a $1000 \mathrm{MW}_{e}$ reactor about $10 \mathrm{MW}$ is required for pumping and refrigeration. In Fig. A-7a, this power is $40 \mathrm{MW}$. The curves show that for $\mathrm{Q}^{\star} \approx 8-10$ the confinement time ratio $\left(\tau / \tau^{*}\right)$ must be $\sim 6$ for $\xi=1$ and $\sim 10$ for $\xi=0.5$. Basically these figures lead to the conclusion that the EBT reactor should be an ignition system. This result is common to that derived in reactor studies based on other confinement concepts.

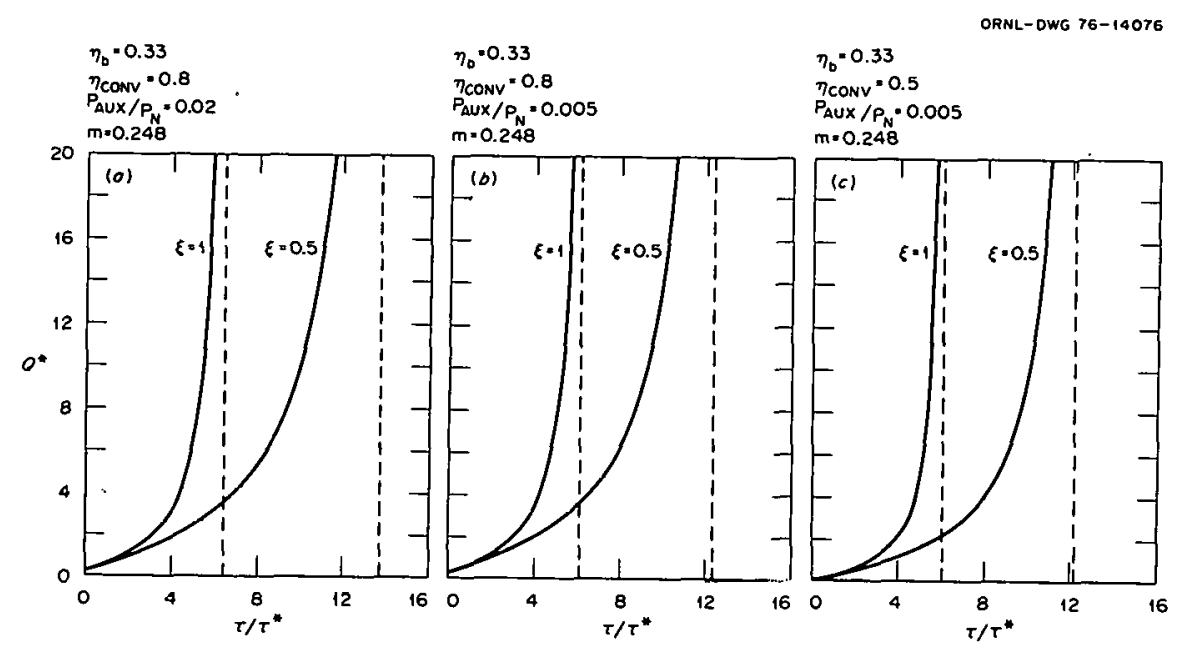

Fig. A-7. $Q^{*}$ versus confinement time.

\subsection{EBT Reactor Cost Scaling}

The EBT reactor is considered to be made up of two constituents: the nuclear island which consists of the blanket and magnets, and the "rest" of the reactor. The cost difference between two reactors with the same thermal output is investigated by comparing the differences in each component separately. In order to facilitate this analysis each component cost is expressed as a fraction of the cost of that component in a reference reactor of interest. The basic requirements of reactor design are used to quantify the range of realistic values rur this ratio. Where possible, optimistic assumptions are used so that the cost of the smaller reactor is reduced to the maximum extent possible. The effect. that the assumptions on the component cost ratios have on the cost of the total reactor is investigated. 


\subsubsection{Basic equations}

If $C_{n}$ is the cost of the nuclear island and $C_{r}$ the cost of the rest of the reactor, and if superscripts $b$ and $s$ refer to the reference system and a scaled down or smaller reactor respectively, then

$$
c_{T}^{b}=c_{n}^{b}+c_{r}^{b}
$$

and

$$
c_{T}^{s}=c_{n}^{s}+c_{r}^{s}
$$

where $C_{T}$ is the total renst of the reactor. Thus,

$$
\frac{c_{T}^{5}}{c_{T}^{b}} \equiv a^{*}-\left(a^{*}-f^{*}\right) r
$$

where

$$
f^{\star}=\frac{c_{n}^{s}}{c_{n}^{b}} ; \quad a^{\star}=\frac{c_{r}^{s}}{c_{r}^{b}} ; \quad r=\frac{c_{n}^{b}}{c_{T}^{b}}
$$

Further, the cost of the nuclear island may be expressed in terms of the blanket cost per reactor section, $C_{b}$, and the magnet cost per section, $C_{m}$, as

$$
C_{n}=N\left(C_{m}+C_{b}\right)
$$

where $\mathrm{N}$ is the number of sections III Llie reactor. Thus,

$$
f^{\star}=\frac{c_{n}^{s}}{C_{n}^{b}}=\frac{N^{s}}{N^{b}} \cdot \frac{c_{m}^{s}}{C_{m}^{b}} \cdot \frac{1+\frac{C_{b}^{s}}{C_{b}^{b}} \cdot \frac{C_{b}^{b}}{C_{m}^{b}} \cdot \frac{c_{m}^{b}}{c_{m}^{3}}}{1+\frac{C_{b}^{b}}{C_{b}^{m}}}
$$

Fquations (21) and (24) are general equations and can be used to compare the costs of two reactors once the component cost ratios have been evaluated. Clearly, each cusl ralio can be extcinded to tnciudu more basic or detailed units if necessary. However, interesting information ahnut cost scaling can be drawn from Eqs. (21) and (24) as they are given here.

\subsubsection{Cust comparisons and optluns available}

Figure A-8 shows the total cost ratio for two reactors as a function of the ratio of the cost of the nuclear island to the total cost for the reference reactor. Consider, for example, two reactors with the same thermal output. The reference reactor is taken to be the 48 section device (major radius $60 \mathrm{~m}$ ) and the simaller reaclur is assumed to bc a 21 section device (major radius $24 \mathrm{~m}$ ). All other reactor parameters, such as mirror ratio, blanket thickness, and magnetic fields are assumed to be equal. 


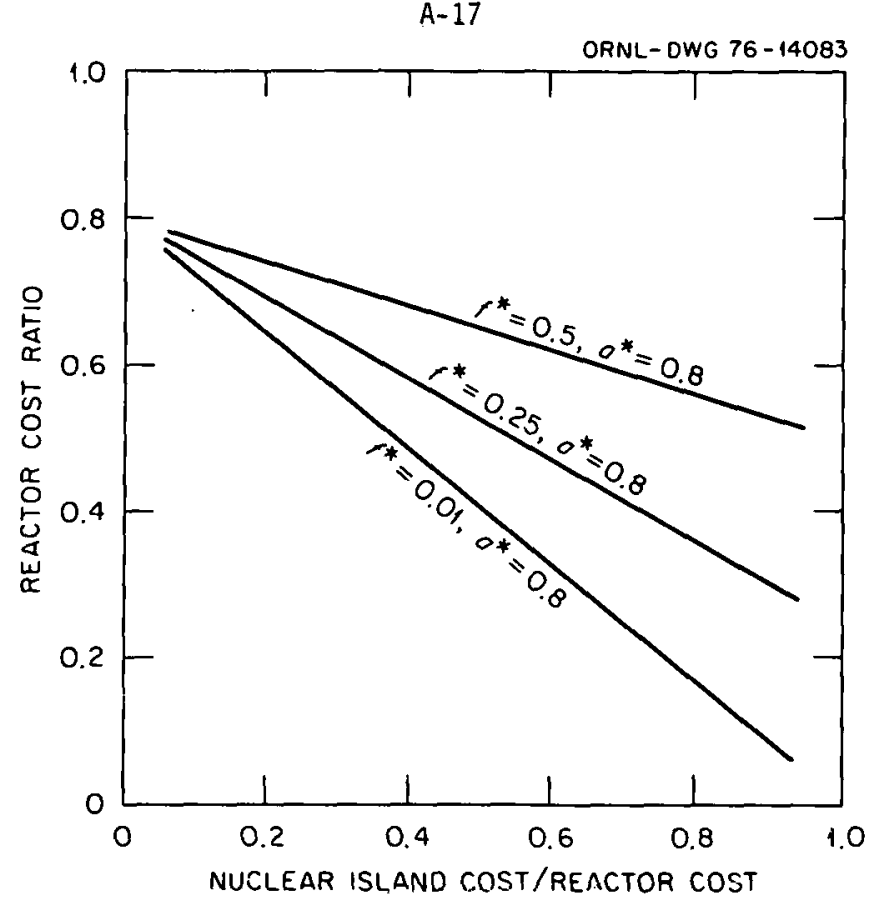

Fig. A-8. Reactor cost ratio versus nuclear island cost ratio for the reference reactor.

Sirice the total thermal output is the same for both reactors, the cost of the auxiliary systems is expected to he essentially the same and thus $a^{*} \simeq 1$. The implicit statement here is that the capital loss for the steam plant, refrigeration, pumping, and plasma heating equipment will be the same. It could be argued that building costs would be reduced for a smaller reactor, but as discussed elsewhere, these costs amount to a small fraction of the total capftal cost. Note that any additional capital costs incurred due to the complexity of remote maintenance of a smaller major radius reactor are neglected by assuming $a^{\star} \simeq 1$. In order to be optimistic, the cost variation for $a^{\star}=0.8$ is investigated.

The smaller reactor costs a factor of two less than the larger one only if $r>0.375$. For the expected values of $r$ for the reference reactor of $0.4-0.6$, say $r$ is 0.4 , then $f^{*}$ needs to be $<0.01$ and if $r$ is 0.6 , then $f^{*}$ needs to be $<0.3$ (Fig. A-8) to achieve the same factor of 2 in cost reduction.

The variation in the muclear island cost ratio, $f^{\star}$, is shown in Fig. A-9 for various magnet cost ratios. It is unlikely that the cost of a magnet will be reduced for a smaller reactor relative to a larger reference case. In fact, since the magnets will tend to be of larger diameter to allow for reactor assembly and maintenance and will carry larger currents in order to produce the same magnetic field in both cases, it is almost certain that an individual magnet will be more expensive. The variation in $f^{\star}$ for magnet cost ratios of 1.0 and 0.8 is examined, and results are probably optimistic. The curves in Fig. A-9 are plotted for various values of the blanket cost ratio. Since reactors with the same mirror ratio are being compared, section lengths are expected to be about the same in both cases; therefore, the blanket cost is expected to be essentially unchanged. The upper two curves (cost ratios 1.2 and 0.8 ) are probably the realistic ones. The blanket cost/magnet cost ratio for the reference reactor is expected to be $\sim 0.2-0.3$. For realistic magnets and blankets the nuclear island cost ratio, $f^{\star}$, will be $\sim 0.4-0.5$. In fact, the only conditions under which $f^{\star} \leq 0.3$ are those where the blanket cost/magnet cost ratio for the reference reactor is 0.4 and the blanket cost ratio is 0.1 . This situation is considered to be unrealistic. From Fig. $A-9$ for $f^{*}=0.4-0.5$ it is found that halving the number of reactor sections, reducing the major radius, and increasing the wall loading to keep the reactor output at the same value is beneficial only if most of the reactor $\cos t(>75 \%)$ is due to the nuclsar island cost itself. 


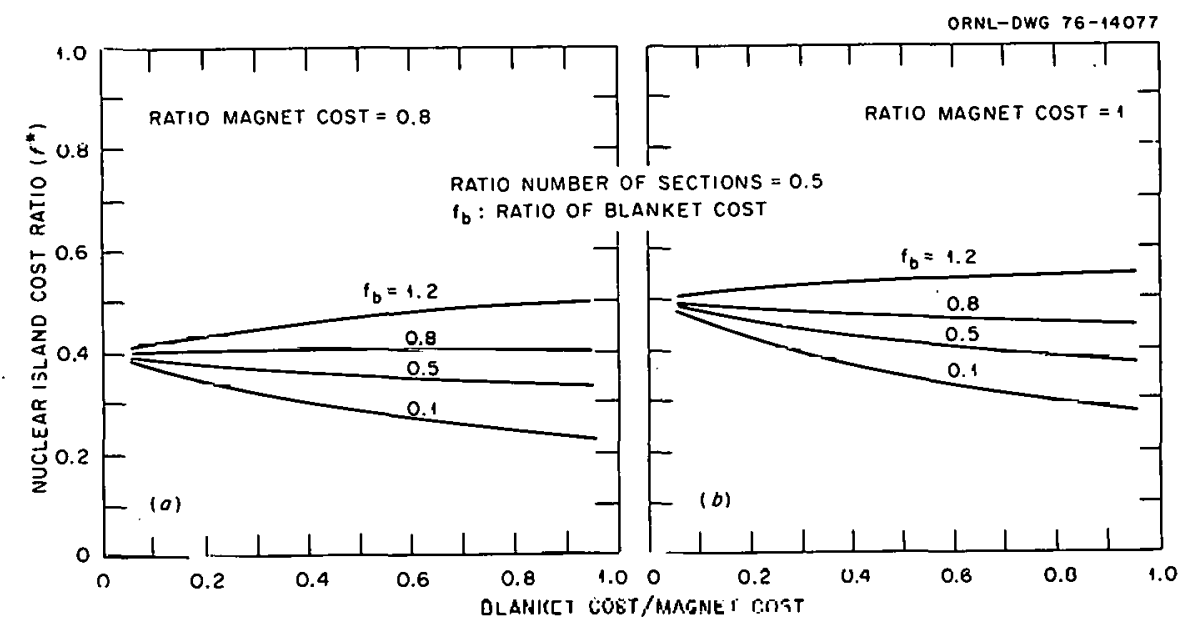

Fig. A-9. Nuclear island cost ratio versus blanket cost/magnet cost ratio.

Equations (21) and (24) can be extended to compare reactor cost variations which accrue due to changes in magnet cost in a given reactor by specifying $N^{s}=N^{b}$ and $C_{b}^{S}=C_{b}^{b}$ (same number of sections and the same blanket cost). The resultant values of $f^{*}$ are shown in Fig. A-10. It is evident that when the nuclear island cost/reactor cost ratios ( $r$ ) are $0.4-0.6$ and the blanket cost/magnet cost. ratios are $0.2-0.3$, a $25 \%$ change in the magnet cost results in $f^{*}$ of $\sim 0.8$. Th1s results (Eq. 21 ) in a new reactor cost of $\sim 0.88-0.92$ times the reference reactor cost. For a $50 \%$ change in the magnet cost, the new reactor cost is $\approx 0.76-0.84$ times the reference.

From these analyses it is concluded that EBT reactor systems are such that the optimum ronst. reactor is not dependent on reactor size in an obvious way. The cost/ $\mathrm{kW}$ is not a strong function of size and there are many construction, engineering, and maintenance benefits gained by high aspect ratio operation. Secondly, if reactor cost optimizations are to be attempted in the future, considerable attention should be given to components outside the nuclear island since they have significant impact on total plant costs. 


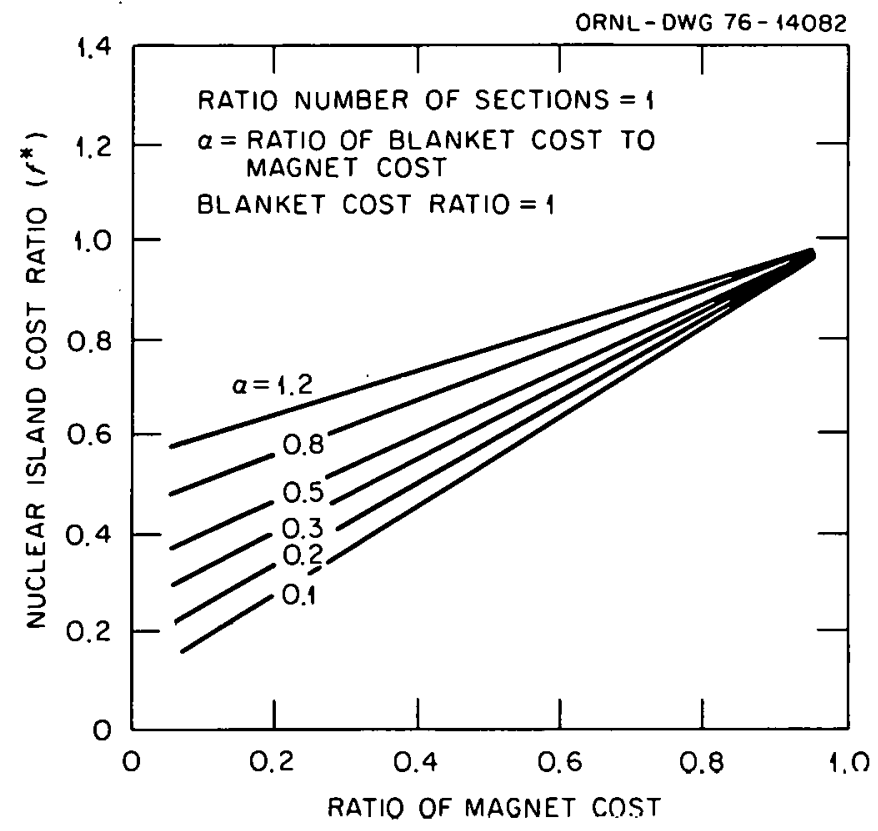

Fig. A-10. Nuclear island cost ratio versus magnet cost ratio for the same reactor.

\section{REFERENCE}

1. A Study of a Utility System Interface with Fusion Reactor Phase I Report, Bechtel Corp. Scientific Development, EPRI RP 547-2. 


\section{INTRODUCTION}

The ELMO Bumpy Torus Reactor (EBTR) is the first reactor design study based on the bumpy torus confinement concept. In the early stages of the EBTR study, power production, technology, and engineering considerations (discussed in Appendix A) primarily were used to establish the EBTR characteristics and operating point. Refinements in plasma engineering and plasma physics have resulted in a revised system description. In this appendix the plasma physics considerations that determine the operating point are discussed. Specific considerations are: 1) particle orbit behavior and understanding of efficient use of the plasma chamber volume, and 2) plasma stability and equilibrium.

The viability of EBT as a reactor requires the stable confinement of a plasma with densities about $10^{20} / \mathrm{m}^{3}$ and temperatures on the order of $10-15 \mathrm{keV}$. These parameters correspond to a plasma $\beta$ of $20-40 \%$ in a moderate magnetic field. For a high beta, steady state system, the plasma equilibrium, particle orbits, ambipolar electric field, and transport phenomena are closely coupled to one another and adequate treatment of any one requires consideration of the others. Specifically, the interrelations are: 1) equilibrium magnetic fields depend on the pressure profile, 2) guiding-center drift orbits depend on the equilibrium magnetic field and ambipolar electric fields, and 3) transport rates, which together with sources and sinks determine the profile, depend on the guiding-center drift orbits. A self-consistent treatment of this coupled set of problems for a large-scale EBT reactor, while being vigorously pursued, is as yet unavailable. Here intermediate and/or partial answers are obtained in order to proceed progressively into the full self-consistent treatment.

\section{EQUILIBRIUM AND DRIFT SURFACES}

Efficient use of the volume of an EBT reactor is proving to have a greater impact upon the design assessment than was expected. The shielding and blanket which surround the chamber in the reactor make the problem more difficult than that in EBT-I. The essence of the problem is that toroidal effects cause the plasma to be shifted inward toward the major axis.

Here this problem is discussed and several solutions are suggested. The first point is that modification of the present inner wall design so that it more nearly follows field lines (as in EBT-I) makes the coil structure the limiting factor. Such a modification would increase the usable volume in EBTR-48 hy ahnut ?5\%. Tho second point is that the coil-blarikel-shield structure can be modified to increase the plasma volume. Here one can either make the aperture eccentric to the coil axis or increase the coil radii by about $25 \mathrm{~cm}$. Either procedure would permit a plasma of the required size.

Finally, there is the possibility of supplementary coils. This is a very promising but relatively unexplored area. One class of supplementary coils was considered for EBT-I, but in that case the cost/benefit ratio was low. This is probably not the case for large devices with different classes of supplementary coils. This topic is being pursued vigorously in the EBT-II design study. The results of these calculations will be used to an advantage in the EBT reactor study. The notion of supplementary coils holds out the promise of high volumetric efficiency for a machine which includes a relatively small number of sectors (perhaps as low as 24).

The spatial position of the hot electron annuli is critical to efficient utilization of the volume within the vacuum chamber. Macroscopic stability demands that the toroidal core plasma pressure be relatively constant near the minor axis and fall rather rapidly in the region of the annuli. Thus the 
annuli form a set of "guard rings" surrounding the toroidal core. To a certain extent, if the diameter of the annuli can be increased, the usable volume within the chamber can also be increased. The caveat is that the toroidal core plasma must pass through the coils and their supporting structure (shield, blanket, etc.). As will be seen, the present inner wall design reduces the diameter of the annuli. When the diameter of the inner wall near the midplane is increased, the annuli diameter is increased and the coil structure becomes the determining factor.

Experimental observation and numerical computation show that the annuli form near contours of constant $\left|\vec{B}_{\text {vacuum }}\right|$ in the midplane of the device. This is a natural consequence of microwave heating. The annular profiles can be adjusted by profile microwave heating at lower frequencies. No overriding technological difficulties are introduced by assuming that lower frequency microwaves can be used to adjust the position of the annuli. Thus, for a given coil configuration it is sufficient to examine the contours of constant $\left|\vec{B}_{\text {vacuum }}\right|$ to determine the maximum diameter of the annuli.

Figure $B-1$ shows these contours of $\left|\vec{B}_{\text {vacuum }}\right|$ for $E B \mid R-48$ as well as $\left|\vec{B}_{\text {vacuum }}\right|$ in the equaliorial plane versus distance from the minor axis for EBTR-48. (The magnetic field was produced assuming filamentary coils, which is sufficient for our purposes here.) Figure B-2 shows the corresponding data for EBTR-24. It will be noticed that the contours are nearly concentric circles. The center of these circles is shifted away from the minor axis toward the major axis. Using a simple approximation for the vacuum magnetic field of a bumpy torus, one finds that the magnitude of this shift is given by

$$
S \cong \frac{2}{\pi}\left(\frac{M+1}{M-1}\right) \frac{d}{N}
$$

where $M$ is the mirror ratio, $N$ is the number of coils, and $d$ is the separation between the coils. The inward shift for EBTR-24 is slightly in excess of three times that of EBTR-48 because both $M$ and $N$ are smaller. While one can reduce this shift in EBTR-24 by increasing the mirror ratio, it seems likely that efficient use of the chamber volume for a 24-sector machine can only be achieved by introducing supplementary coils. For this reason, atzention ts focused on EBTR-48 in line remainder of this appendix.

For a mirror field, the field lines naturally compress as one goes from the midplane to the coil plane, as shown in Fig. B-3. To lowest order the plasma follows the field lines. Notice that for the inner wall design in Fig. B-3, many of the field lines which pass through the coil aperture intercept the lateral wall of the chamber. Consequently, the diameter of the plasma is limiled by the purtiuin of the inner wall in the midplane rather than in the coil plane. Figure B-4 shows an inner wall configuration where the coil aperture limits plasma diameter. As indicated in Fig. B-1, this allows an increase in the plasma diameter. The corresponding increase in the usable volume of the container is about $25 \%$. This inner wall configuration is somewhat arbitrary. The important feature is that the part of the wall closest to the major axis follows a field line as closely as is practical.

Figure B-5 shows a case in which the coil structure aperture has been shifted inward approximately $25 \mathrm{~cm}$ (its diameter was held constant). This case allows virtually $100 \%$ utilization of the aperture. The notion here is that it may be technologically feasible to have a nonuniform layer of shield and blanket within the coil throat. An alternative is simply lo increase the radius of the coll by about $25 \mathrm{~cm}$ while keeping the thickness of blanket and shield constant. Either method would lead to the plasma size required for EBTR-48.

The drift motion of single particies is now considered. Here finite beta and the ambipolar electric fields must be taken into account. As will be seen, only a small region of velocity space is lossy. The bulk of the particles in the toroidal core and annuli are well contained. 

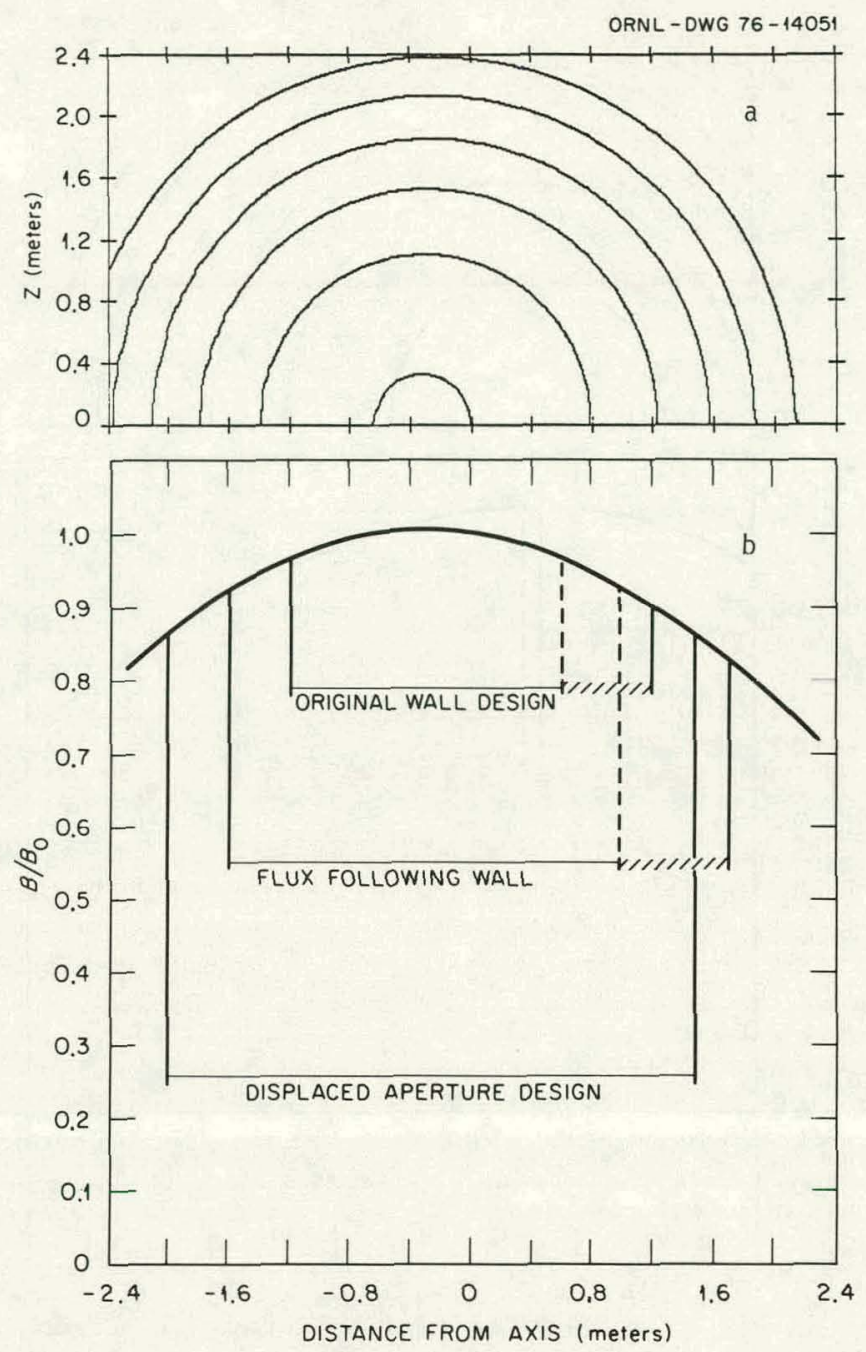

Fig. B-1. Contours of constant $B$ in the midplane for EBTR-48 (a) and $B / B_{0}$ vs distance from the minor axis (b). $B_{0}$ is the value of $B$ on the minor axis in the midplane. Fig. $B-1 b$ indicates the positions of the inner wall for the severāl designs shown in Figs. B-3, B-4, and B-5. The non-cross hatched parts indicate the maximum usable portion for the three designs. The displaced aperture inner wall design would produce nearly $100 \%$ maximum volumetric efficiency.

The general features produced by finite beta and ambipolar electric fields can be most easily understood in terms of the local drift velocity

$$
\vec{V}_{D}=\frac{\vec{E} \times \vec{B}}{B^{2}}+\frac{1}{\Omega}\left[\frac{1}{2} V_{\perp}^{2} \hat{n} \times \nabla \ln B+V_{\|}^{2} \vec{k}\right]
$$

where $\Omega=e B / m$ is the cyclotron frequency, $\hat{n}=\vec{B} / B$, and the curvature vector is given by

$$
\vec{k}=\hat{n} \times \partial \hat{n} / \partial s .
$$

Notice that for low energy $\left(\operatorname{small}_{1} \mathrm{v}_{\perp}\right.$ and $\left.v_{\|}\right)$the particle drift is dominated by the $\vec{E} \times \vec{B}$ drift. In the desirable mode of operation in EBT-I, the electrostatic potential, $\phi$, is such that e $\phi / T_{i}$ is on the order of unity. Since the distribution of low energy particles is approximately Maxwellian, the bulk of the particles have energies below several times $T_{j}$; consequently, the bulk of the particles' motion is dominated by the $\vec{E} \times \vec{B}$ drift. 
B-4
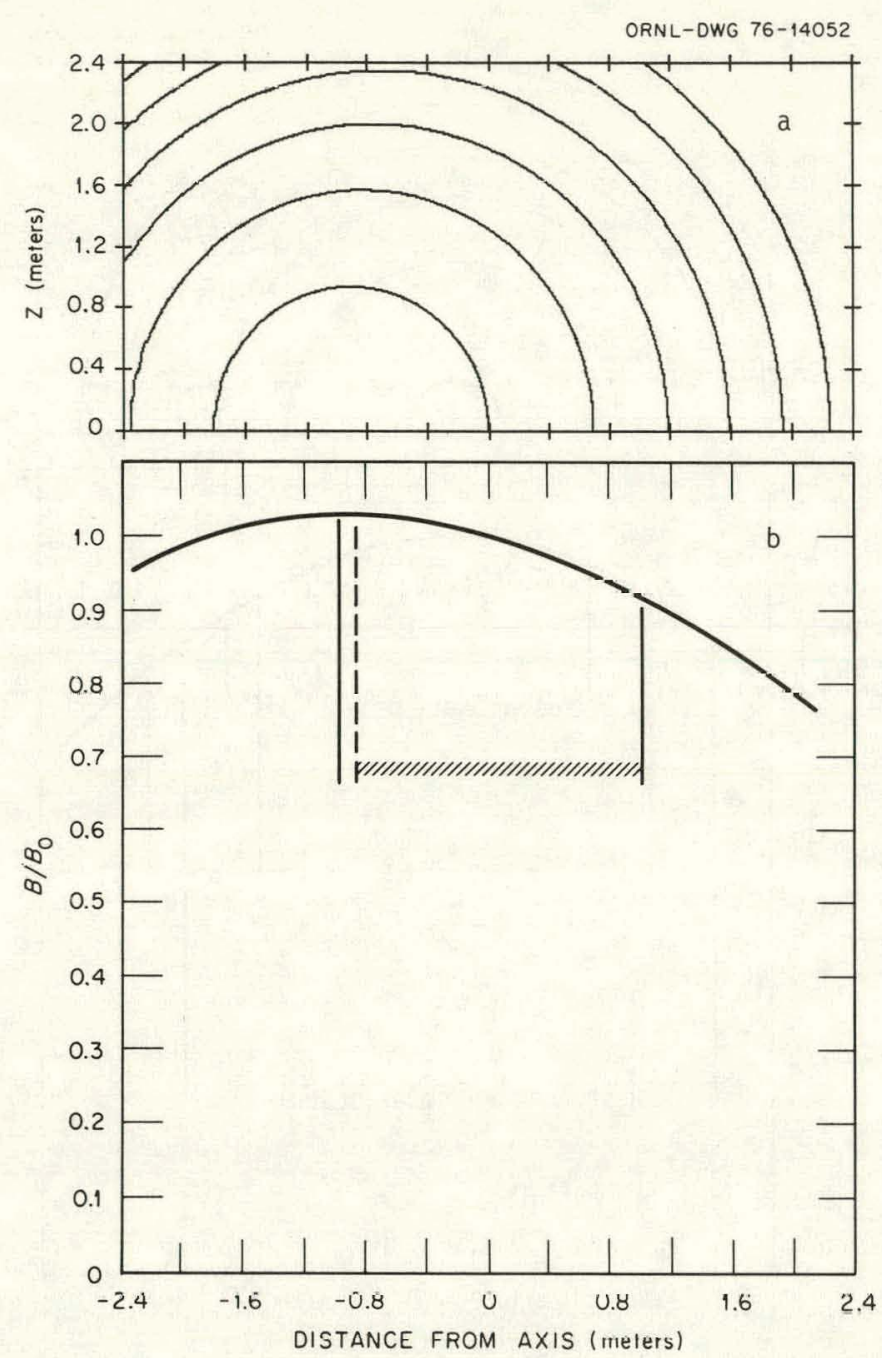

Fig. B-2. Contours of constant B in the midplane for EBTR-24 (a) and B/Bo vs distance from the minor axis (b). The exceedingly small usable volume can be improved somewhat by changes in mirror ratio and wall design.

ORNL-OWG $76-14053$

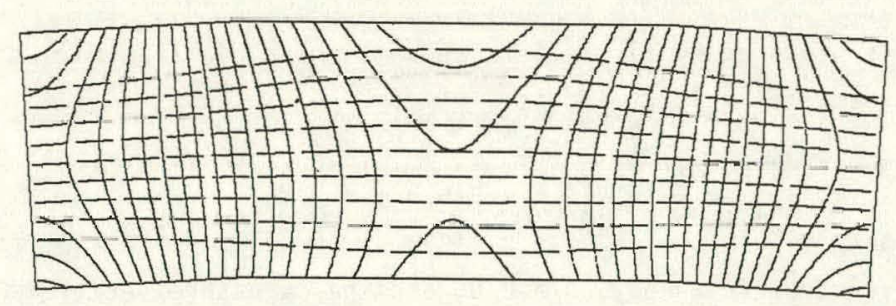

Fig. B-3. $|B|$ contours and field lines in the equatorial plane for a sector of EBTR-48 with the original inner wall design. The boundary is the inner wall (and the coil planes). Solid curves show contours of constant B. Dashed lines represent the field lines. 


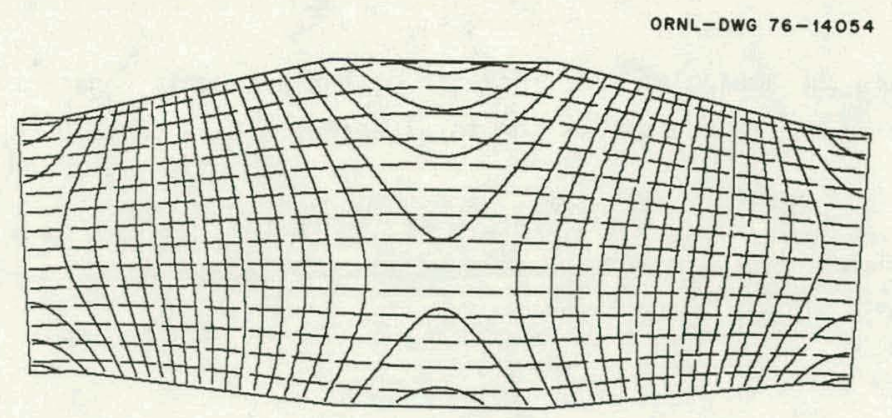

Fig. B-4. $|B|$ contours and field lines for EBTR-48 in the equatorial plane for a "flux-following" inner wall. The aperture in the coil plane has been fixed and the inner wall arranged to approximately follow the field lines. The precise detail of such inner walls remains to be specified.

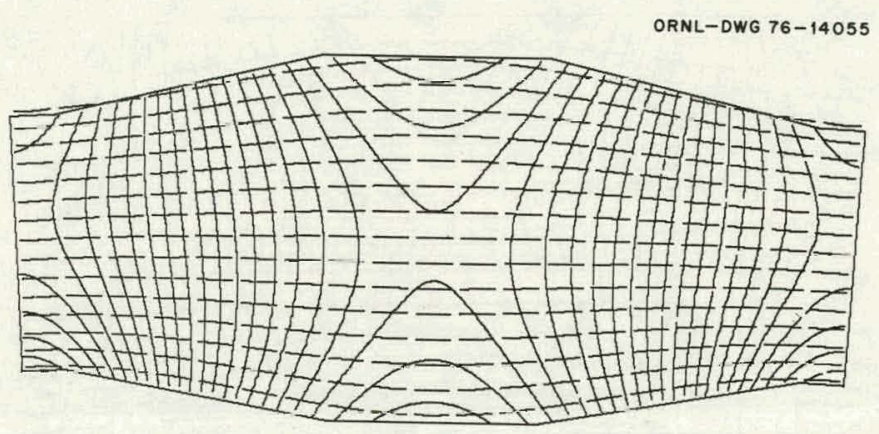

Fig. B-5. $\mid$ B|contours and field lines for EBTR-48 in the equatorial plane for a displaced aperture. The aperture has been moved toward the major axis so that is projection along field lines into the midplane lies on $|B|$ contours. This wall design would provide the necessary size plasma required for EBTR-48.

On the other hand, fur high energy particles ( $v_{\perp}$ and $v_{\|}$large), the $\vec{E} \times \vec{B}$ drift is negligible and their drift motion is dominated by the gradients and curvature of the magnetic field. Figure B- 6 shows the effect of a high beta annulus on the magnetic field. Typically, the gradients in B are strongly affected, as evidenced by changes in the $|\vec{B}|$ contours. The field line shapes and therefore their curvature, $\vec{k}$, are relatively unaffected by finite beta. Thus we see from Eq. (2) that finite beta has its greatest effect upon particleswith $v_{\|}=0\left(a 11 v_{\perp}\right)$ and relatively little effect upon those with $v_{\|}=v\left(v_{\perp}=0\right)$.

From the above discussion one can conclude that high energy particles with $v_{\|} \simeq \underline{v}$ are relatively unaffected by either the electric field or the finite beta. Particles with high $v_{\|}$tend to be poorly confined in a vacuum field as well, as may be seen by referring to Fig. B-6. Notice that the curvature of the field lines in the coil plane is opposite to that in the midplane. Consequently, particles with high $v_{\|}$sample both signs of the curvature. The time-averaged curvature can be nearly zero. Hence, the poloidal drift motion produced by the mirror-like field can be very small for these particles and the toroidal curvature causes them to drift nearly straight up (or down) into a material wall.

To carry out detailed evaluation of particle orbits, it is convenient to introduce the magnetic moment, $\mu$, and the longitudinal adiabatic invariant, $\mathrm{J}$ : 


$$
\mathrm{J}=\oint \mathrm{v}_{\|} \mathrm{d} l
$$

Under the assumption that the ambipolar electrostatic potential, $\phi$, is constant along field lines, the kinetic energy is given by $\varepsilon-\mathrm{e} \phi$ where $\varepsilon$ is the total energy. As a consequence

$$
\frac{1}{2} m v_{\|}^{2}=\varepsilon-\mathrm{e} \phi-\mu B
$$

and

$$
J=\sqrt{2 / m} \oint d l \sqrt{\varepsilon-e \phi-\mu B} .
$$

Since $\varepsilon, \mu$, and $J$ are conserved quantities (adiabatical1y), the particle must lie on a surface of constant $\mathrm{J}$ for fixed $\varepsilon$ and $\mu$. Particle drift surfaces may be determined by calculating $\mathrm{J}$ on a grid in the midplane for fixed $\varepsilon$ and $\mu$ and from this the contours of constant $J$ can be determined.

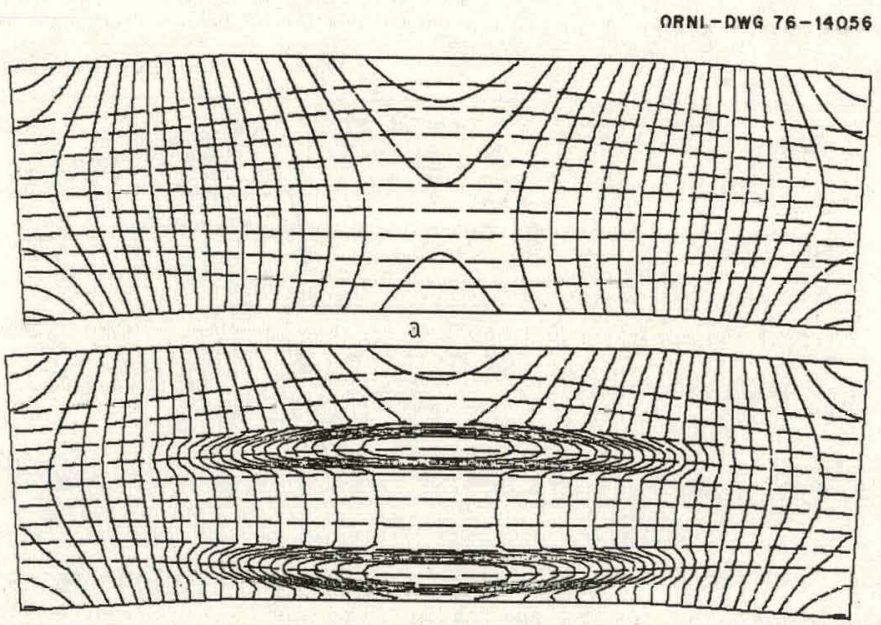

h

Fig. B-6. $|B|$ contours and field lines for EBTR-48 with (b) and without (a) finite $\beta$. The minimum in $|B|$ produced by the annulus is clearly visible in Fig. B-6b.

To make a detailed calculation, a definite magnetic equilibrium and ambipolar potential must be chosen. Figure B-7 shows the annular pressure profile selected for evaluation along with its effect upon B. While one could consider an additional toroidal core of comparable beta (e.g., $\sim 50 \%$ ) the important feature is the outer gradient in B. Here the present inner wall design has been used. Inis design, as discussed previously, poses severe restrictions on the annular diameter. Figure B-8 shows the ad hoc electrostatic potential. Notice that the potential has non-zero gradients in the outer portions of the annuli. This is in keeping with experimental observations from EBT-I. This has the consequence that contours of constant $B$ and $\phi$ are nearly identical so that the electric field has little effect upon the shape of the drift surfaces for $v_{\|} \simeq 0$.

An important question is what particle drift orbits are contained within the chamber. A measure of this is provided by the area (in the midplane) of the last closed contour which does not strike the wal1. This notion is illustrated in Fig. B-9. Volumetric efficiency, F, is defined by

$$
F=\frac{\text { area of last closed contour }}{\text { area intercepted by the Tiner }} \text {. }
$$




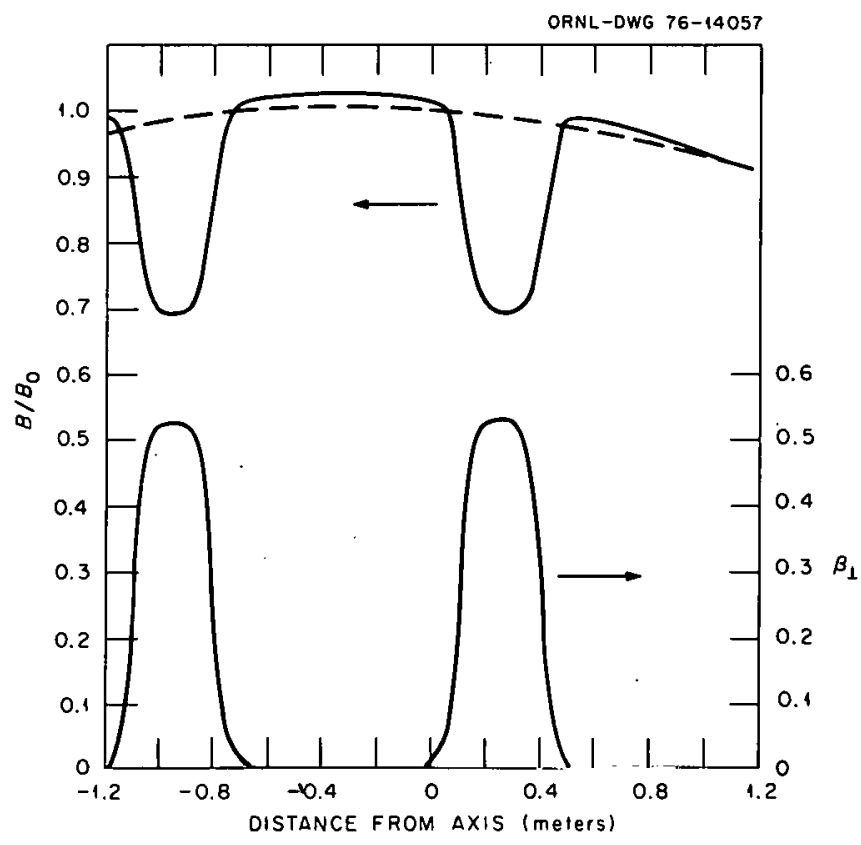

Fig. B-7. Magnetic field vs distance from the minor axis in the midplane for the vacuum field (dashed) and finite $B$ field (solid), The lower curve shows $p_{1}$ scaled by the vacuum magnetic pressure $\frac{1}{2} \mathrm{~B}_{0}^{2} \quad\left[\right.$ i.e., $\left.B_{\perp} \equiv \mathrm{p}_{\perp} /\left(\frac{1}{2} \mu_{0} B^{2}\right)\right]$.

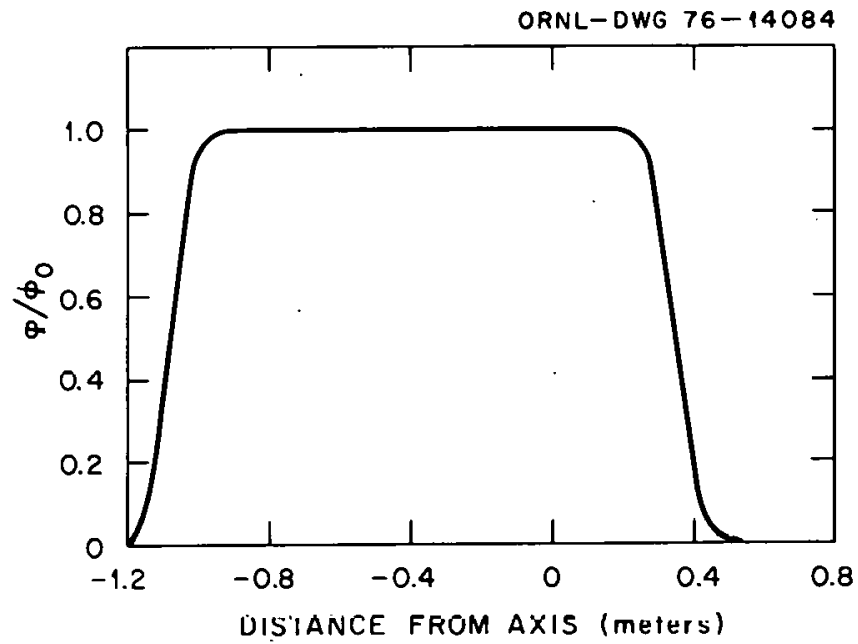

Fig. B-8. The ambipolar electrostatic potential, $\phi$, vs distance from the minor axis. Comparison with Fig. B-7 will reveal that non-zero gradients in $\phi$ only occur in outer positions of the annulus. 


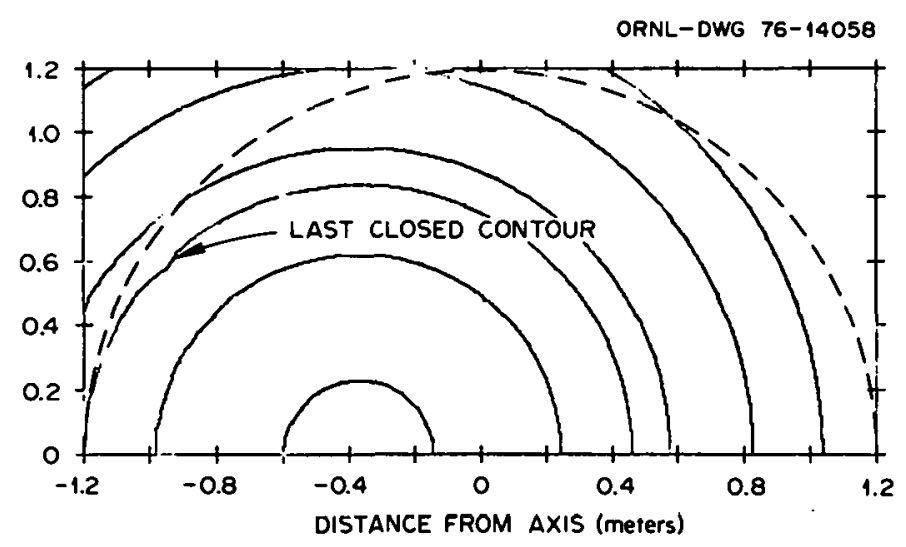

Fig. B-9. Contours of constant $j$ in the midplane (solid) and the inner wall (dashed). Notice that the "last closed contour" is tangent to the inner wall.

Since the particle orbits depend upon $\varepsilon$ and $\mu$, so does the volumetric efficiency. The results are displayed in terms of the more intuitive variables $v_{\|} / v$ and the (kinetic energy)/|e $\phi_{0} \mid$ measured on the minor axis in the midplane. (When plotted versus $v j v$, an isotropic distribution is constant. Thus, using $v / v$ facilitates estimating the fraction of particles which may lie in a loss reqion.) Figure B-10 shows the volumetric efficiency for ions and electrons. The differences between the two species are caused by the sign of the charge. At some points in velocity and configuration space, the electric field can nearly cancel the gradient $B$ and curvature drifts, leading to poorer confinement. This is most evident in Fig. B-10a for ions.

The details of the surfaces of volumetric efficiency, such as shown in Fig. B-10, depend upon details of the inner wall design as well as the magnetic equilibrium and ambipolar electric field. Thus the depression seen for the ions in Fig. B-10 is not universal. There are three features which are relativêly independent of these details:

(1) low energy particles are well confined,

(2) well-trapped particles are well confined,

(3) high energy particles with high $v / \mu$ are the worst confined.

The notion of supplementary coils was considered for EBT-I to improve the confinement of this last class of particies. Ihere are a number of possible supplementary coil configuratinns currently under investigation. Only those factors motivating the coil design are discussed here.

For particles with $\dot{\mu}=0\left(v_{\|}=v\right)$, the longitudinal adiabatic invariant can be written as

$$
J=\sqrt{2 / m} \sqrt{\varepsilon-e} \phi \oint d \dot{l} .
$$

For highly energetic particles we may neglect the $\phi$ deperidence and obtain

$$
\mathrm{J} \propto \oint \mathrm{d} \ell .
$$

Thus, for these particles, $J$ is proportional to the length of the field line. Because of the tendency for the field lines at larger major radij to be longer, an inward shift of the constant $J$ surfaces results. 

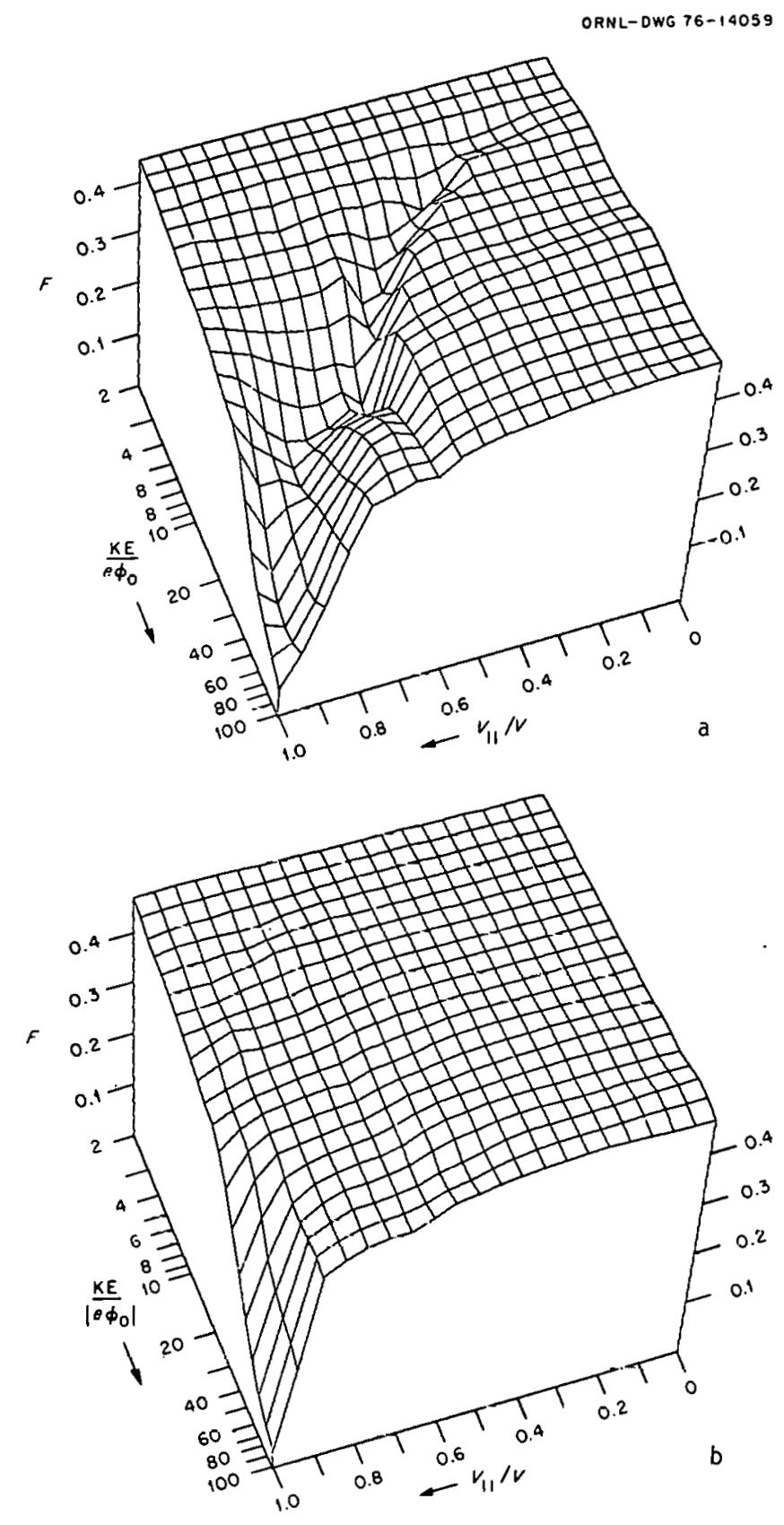

Fig. B-10. Ion (a) and electron (b) volumetric efficiency, $F$, vs $v \mid \mathbb{v}$ and kinetic energy scaled by eфo. The magnetic and electric fields are those indicated in Figs. B-7 and B-8. The details of the figures depend upon the details of the inner wall design, electric field, and finite beta field. The more universal features mentioned in the text are clearly present. The maximum vulumetric efficiency here is slightiy less than 0.5 . By using the inner wall design of Fig. B-5 this could be increased to unity.

By adding supplementary coils, the inner field line length can be made equal to the outer field line length. The so-called M\&S configuration ${ }^{1,2}$ is an example of such a configuration, but requires a large number of coils. The focus of the EBT-II supplementary coil design studies is on more economically attractive coil configurations which produce nearly the same effect upon field line 
length. The configurations under consideration should also allow a more symmetrized set of $\left|\vec{B}_{\text {vacuum }}\right|$ surfaces. This would avoid the use of the eccentric or larger main coils mentioned earlier in this appendix. Indeed, supplementary coils may even make a design with a small number of coils (e.g., 24) attractive.

\section{MACROSCOPIC STABILITY}

The viability of EBT as a reactor requires the stable confinement of a plasma with $n \sim 10^{20} / \mathrm{m}^{3}$ and $T \sim 10-15 \mathrm{keV}$. These parameters correspond to a plasma $B$ of about $20-40 \%$ in a magnetic field of $25 \mathrm{kG}$ on the minor axis in the midplane. However, in the vacuum field produced by the EBT coils, such a plasma is grossly unstable according to either magnetohydrodynamic (MID) theury ur Vlasov theory, both of which predict macroscopic flute-like drift instabilities having grnwth rates of lows than a microsecond, leading to correspondingly short containment times. Such instabilities have been observed in uller bumpy tor and appear to exist in EBT in the C-mode.

Crucial to the stability of the EBT plasma is the presence of a relativistic hot electron population, with $\mathrm{T}_{\mathrm{e}} \sim 100 \mathrm{keV}$, but with a required density of only about $\mathrm{n}_{\mathrm{e}} \sim 10^{18} \mathrm{~m}^{-3}$, or a small fraction of the total electron density. This hot electron population (annuli) is immune to the instabilities mentioned above because of the fast drift velocities and large Larmor radii of its particles. By modifying the magnetic field, it. also provides stability for the rest of the plasma, composed of warm ions and electrons. Thus the simplest model for discussing the stability of the EBT plasma is one with three components: hot electrons, warm electrons, and warm ions. One can treat first the instabilities of the hot electrons, and then the instabilities of the warm species. The effort to date has been concentrated on macroscopic instabilities, those which produce large particle transport and would be fatal to a reactor. Microinstabilities, which could lead to enhanced transport, are not included in this discussion.

\subsection{Stability of the Hot Electron Annuli}

Although the hot electrons in EBT form annuli about the magnetic axis, this onnfigurationi is llut necessary for either their stability or that of the bulk plasma. What is required is that their temperature be high enough and the magnetic field gradient produced by their diamagnetism be large enough to achieve a $\nabla B$ drift frequency which exceeds the ion cyclotron frequenry. When this condition is reached, analysis of the Vlasov-Maxwell equations shows that hot electron drift waves are stabilized, apparently by interference between the responses of the fast drifting electrons and the slowly drifting ions. $^{3}$ As the hot electron temperature or density is reduced, these waves leculle unstable, and at still lower temperature, where the MHD and guiding center theories are applicable, they become the unstable flutes and ballooning interchanges predicted by these theories. Thus, stable operation of a reactor will require that the hot electrons achieve a temperature comparable to that in the present device and a pressure profile of sufficient steepness to produce large $\nabla B$ drifts.

Near the outer edges of the annuli, the hot electron temperature is not high enough to stabilize these instabilities. However, in the present EBT the cavity shape is such that this region is linetied, and line-tying is an effective stabilizing mechanism for these modes. We helieve that the outer edge of a reactor plasma must also be line-tied, unless a supplementary coil system is used to create a local minimum in the vacuum B-field. Present theory does not allow a definitive answer as to how larae the line-tied region must be. The experiment gives a practical answer in that the location of the annuli can be varied by changing the magnetic field strength and hence the resonant zone in which the annuli are formed. The outer edge always extends to a line-tied region, but in general this region is quite small. 


\subsection{Stability and Beta Limitations of the Bulk Plasma}

In the vacuum field of EBT the warm ion and electron populations forming the bulk plasma are likewise subject to unstable drift and MHD modes at frequencies characteristic of their $\nabla B$ drift. The hot electron annuli stabilize these modes by a very interesting mechanism. The hot electron diamagnetic currents act as "invisible coils" colocated with the bulk plasma and create a region of minimum average $B$ in which $\Phi d l / B$ decreases with radius. In this region a stable bulk plasma can be established and then stably extended to the magnetic axis, even though the minimum average $B$ region does not extend to the magnetic axis. Figure B-11 shows the location of the hot electron plasma, the region of decreasing $\emptyset \mathrm{d} \ell / B$, and the stably confined bulk plasma. In general, one finds that the bulk plasma beta can approximate that of the annuli before instability occurs.
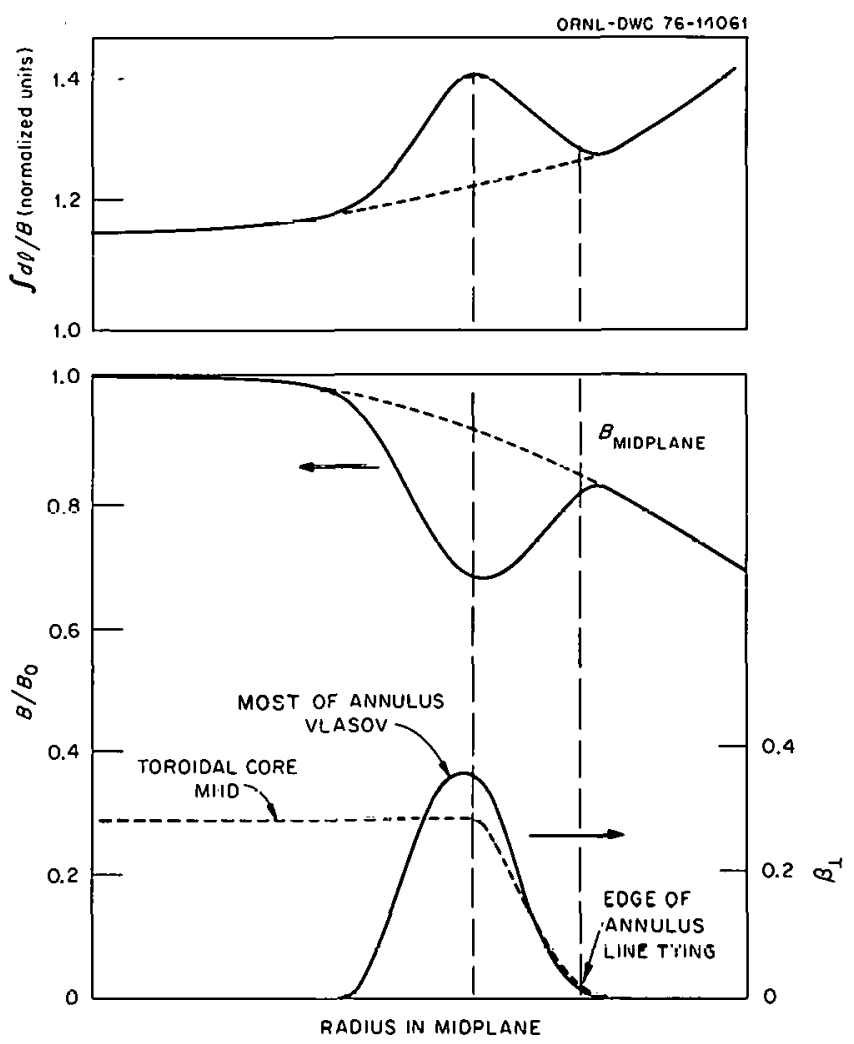

Fig. B-11. $d \mathrm{~d} \ell / \mathrm{B}$, magnetic field, and pressure vs distance from the minor axis in the midplane for the vacuum ficld (dashed) and finite $\beta$ field (solid).

Tó justify these results, several steps of analysis are required. First, all three species are considered using Vlasov equations, but here only simple geometries such as planar or cylindrical can be considered. It is found that because of their fast drift velocity and low density the hot electron terms in the dispersion relation for localized electromagnetic drift waves are negligibly small. However, the hot electrons do modify the magnetic fields seen by the other species and hence the equilibrium configuration. When the hot electron beta becomes large enough to reverse the direction of $\nabla B$ relative to the curvature (or gravity) these drift waves are stabilized. Repeating this calculation using the MHD or guiding. center equations in the same simplified geometry, and considering the hot electrons as invisible currents which affect the equilibrium but not the stability, one finds the same results as 
for the Vlasov equations, except that when the $\nabla B$ drifts are reversed all localized MHD modes are stabilized.

The simple way in which the hot electron terms drop out of the dispersion relation gives one confidence that the same would occur if one could apply the Vlasov equation to the real bumpy torus geometry. The expected agreement between the Vlasov analysis and the MHD analysis then leads to the next step: the guiding center equilibrium equations are solved numerically for mirror-confined annuli together with a scalar pressure bulk plasma in a bumpy cylinder geometry. Then the hot electrons are treated as rigid in a $\delta W$ MHD stability analysis of the bulk plasma. Figure B-12 shows that once a well has been established by the annuli (at $\beta_{A} \sim 5-15 \%$ ) a beta of the bulk plasma comparable to, or higher than, the beta of the annuli can be achieved which is stable to all modes encompassed by the guiding center or ideal MHD theories. These modes which limit bulk beta are pressure-driven ballooning interchanges. The last step, extension to full bumpy torus geometry, has been carried out analytically. ${ }^{4,3}$ Because the bumpy cylinder provides an excellent approximation to the bumpy torus, both in equilibrium and stability, the numerical calculation of stability boundaries in the torus is not considered essential.

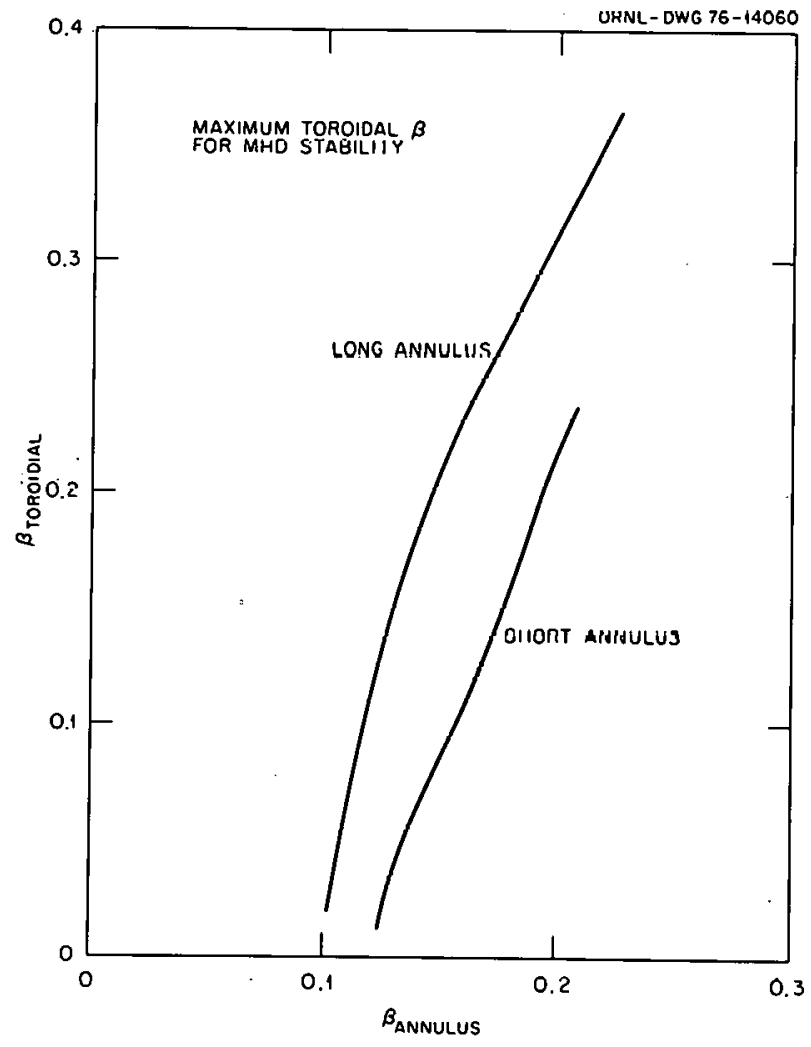

Fig. B-12. Maximum toroidal B for MHD stability. 
Experimental confirmation of these optimistic beta predictions is not possible in the present device because there beta is limited by heating and transport considerations. However, the threshold for stable confinement at $\beta_{A} \sim 8 \%$ is observed, where it marks the boundary between $C$ - and T-modes.

\section{REFERENCES}

1. F. Meyer and H. V. Schmidt, Z. Naturforsch 13a 1005 (1958).

2. G. H. Wolf, Z. Naturforsch 24a. 998-1021 (1969) and references cited therein.

3. G. E. Guest, C. L. Hedrick, and D. B. Nelson, Phys. Fluids 18871 (1975).

4. G. O. Spies and D. B. Nelson, Phys. Fluids $\frac{17}{1865}$ (1974).

5. D. B. Nelson and G. 0. Spies, Phys. Fluids 172133 (1974). 
APPENDIX C

CYLCOTRON RADIATION

\section{INTRODUCTION}

One factor in the economic analysis of the EBTR is the question of how much power is lost from the annulus and bulk plasma as radiation; in particular, the cyclotron losses of the very hot annular electrons must be replenished by the microwave sources sustaining the annuli.

In this approach, because of the complexity of the problem, a simplified theory has been used to estimate the cyclotron losses by assuming slab model geometry with (Maxwellian) temperature, density, and magnetic field uniform within each plasma region but differing between the neighboring bulk and annular regions. General formulae are presented for the total cyclotron power loss and its dependence on annular beta, annular thickness, and first wall reflectivity.

\section{FORMULATION OF RADIATION LOSSES}

The equation governing radiative energy transfer is

$$
n_{r}^{2} \frac{d}{d z}\left(\frac{s}{n_{r}{ }^{2}}\right)=-\alpha s+w_{c}
$$

where $S$ is the spectral intensity into a given solid angle along a path $z, \alpha$ is the absorption coefficient, $w_{c}$ is the cyclotron radiation production rate, and $n_{r}$ is the index of refraction. The electron cyclotron motion principally radiates extraordinary waves which have roughly $n_{r}=1$ as long as their frequencies $(\omega)$ exceed the local plasma frequency $\left(\omega_{p}\right)$.

The cyclotron frequency $\left(\omega_{c}\right)$ in the annulus and midplane is $4.4 \times 10^{11} \mathrm{sec}^{-1}$ and in the mirror throat $\omega_{c}=7.92 \times 10^{11} \mathrm{sec}^{-1}$. If a bulk average density $n_{\mathrm{e}}=1.2 \times 10^{20} \mathrm{~m}^{-3}$ is used to compute the bulk plasma frequency, one finds $\omega_{p}=6.2 \times 10^{11} \mathrm{sec}^{-1}$. Thus the annulus radiation will not readily propagate through the bulk plasma. (The same is true of the bulk radiation in the midplane.) If the bulk density is not flat (e.g., a parabolic profile), the peak plasma frequency could cut off the first harmonic as well. The equation of transfer will use $n_{r}=1$ for now but allow for spectral modification at $\omega_{c}$ and $2 \omega_{c}$.

Generally, $S=S(\omega, \theta)$ since cyclotron emission and absorption are anisotropic. However, $\alpha(\theta)$ is a fairly slow function of $\theta^{1}$ so the spectral flux is approximately

$$
\begin{aligned}
S(\omega) & \cong \int_{0}^{\pi / 2} d \Omega \cos \theta S(\omega, \pi / 2) \\
& =\pi S(\omega, \pi / 2) .
\end{aligned}
$$

So, one can do the calculation for radiation propagating perpendicular to the magnetic field lines only.

An additional complication arises when treating the radiation reflected off the walls of the . containment vessel. Here, a simple treatment is considered, which examines light propagating purely radially through the plasma and neglects details of the toroidal vacuum chamber. The characteristic cutoff frequencies $\left(\omega^{\star}\right)$ for black body radiation have only a slight dependence on plasma geometry, ${ }^{1}$ so values typical of a slab geometry will be used. 
Setting $n_{r}=1$ and recalling that emission and absorption are related by the black body formula in equilibrium, the equation of transfer is integrated to get

$$
S(2)=B+[S(1)-B] e^{-\int_{1}^{2} \alpha d z} \text {. }
$$

For simplicity one can assume the plasma is a uniform temperature Maxwellian, in equilibrium, but note that anisotropic, non-Maxwellian distributions, sucil as occur in hot electron annuli, may considerably alter these results. The black body intensity above is $B=\frac{\omega^{2} T}{8 \pi^{3} c^{2}}$; points 1 and 2 are where the ray enters and leaves the plasma.

At this point a ray is traced as it bounces back and forth through bulk plasma and the high temperature annular sheath (see Fig. C-1).

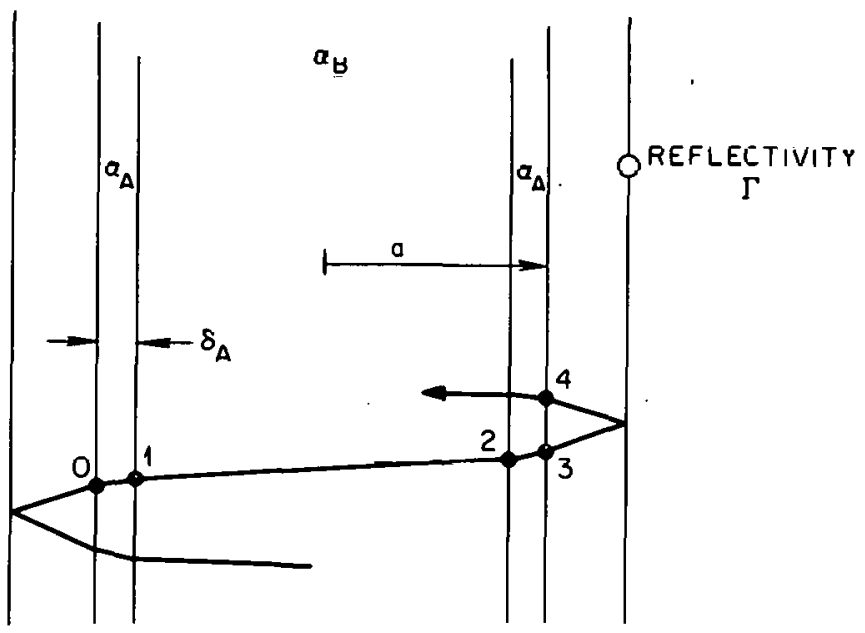

Fig. C-l. Radiation path through bulk plasma and annular sheath. Wall reflectivity is $\Gamma$.

For uniform plasma properties across each section, purely radial propagation, a bulk plasma radius a, and an annulus thickness $\delta_{A}$, one has

$$
\begin{aligned}
& S(1)=B_{A}+\left[S(0)-B_{A}\right] e^{-\alpha_{A} \delta_{A}} \\
& S(2)=B_{B}+\left[S(1) \quad B_{B}\right] c^{-\alpha_{B} 2 a} \\
& S(3)=B_{A}+\left[S(2)-B_{A}\right] e^{-\alpha_{A} \delta_{A}} \\
& S(4)=\Gamma S(3) \\
& S(4)=S(0),
\end{aligned}
$$

where $\Gamma$ is the wall reflectivity. From solution of Eq. (2) the outward flux across the surface of the bulk plasma is

$$
\begin{aligned}
I_{B}(\omega) & \equiv \pi[S(2)-S(1)] \\
& =\frac{\pi\left\{1-e^{\left.\alpha_{B}^{2} a\right\}}\left\{( B _ { B } - B _ { A } ) \left[1-\Gamma e^{\left.\left.-\alpha_{A} 2 \delta_{A}\right]+B_{A}(1-\Gamma) e^{-\alpha_{\tilde{A}} \delta_{A}}\right\}}\right.\right.\right.}{1-\Gamma e^{-\alpha_{A} 2 \delta_{A}-\alpha_{B} 2 a}},
\end{aligned}
$$


and the outward flux across both surfaces of the annulus is

$$
\begin{aligned}
& I_{A}(\omega)=\pi[S(3)-S(4)+S(1)-S(2)] \\
& =\left[1-\Gamma e^{-\alpha_{A} 2 \delta_{A}-\alpha_{B} 2 a}\right]-1 \pi\left\{B_{A}\left(1-\Gamma e^{-\alpha_{A} 2 \delta_{A}}\right)\left(1-e^{-\alpha_{B} 2 a}\right)\right. \\
& +B_{A}(1-\Gamma)\left(1-2 e^{-\alpha_{A} \delta_{A}}+2 e^{-\alpha_{A} \delta_{A}-\alpha_{B} 2 a}-e^{-2\left(\alpha_{A} \delta_{A}+\alpha_{B} a\right)}\right) \\
& -B_{B}\left(1-e^{-\alpha_{B} 2 a}+e^{-\alpha_{B} 2 a-\alpha_{A} \delta_{A}}-e^{-\alpha_{A} \delta_{A}}\right) \\
& -B_{B} \Gamma\left(e^{-\alpha_{A} 2 \delta_{A}-\alpha_{B} 2 a}-e^{-\alpha_{B} 2 a-\alpha_{A} \delta_{A}}+e^{-\alpha_{A} \delta_{A}}-e^{\left.\left.-\alpha_{A} 2 \delta_{A}\right)\right\}}\right.
\end{aligned}
$$

These expressions are very complicated due to the frequency dependence of the absorption and reflection coefficients. For example, a clean first wall of stainless steel has $5=1-7.3 \times 10^{-9} \omega^{\frac{1}{2}}$. However, pumping ports, injection apertures, diagnostics, etc. will reduce the total first wall reflectivity. Moreover, during the first wall lifetime its reflectivity will probably degrade due to severe reactor conditions, so $\Gamma \equiv 0.9$ as the typical value has been assumed. (Results are insensitive to the exact value of $\Gamma$ provided $\Gamma \lesssim 0.99$.)

The function $\alpha(\omega)$ has been published ${ }^{2}$ for $T=10,50$, and $100 \mathrm{keV}$ and is available at ORNL for arbitrary temperatures. To simplify expressions for $I(\omega)$, cutoff frequencies $\left(\omega^{*}\right)$ are defined by $\alpha_{B}\left(\omega_{B}^{*}\right) 2 a \equiv 1$ and $\alpha_{A}\left(\omega_{A}^{*}\right) 2 \delta_{A} \equiv 1$. Since $\alpha(\omega)$ decreases very quickly as $\omega$ increases, the exponential factors in $I(\omega)$ are easily approximated in various frequency intervals. For Case I where $\omega^{\star}{ }_{B}<\omega_{A}^{\star}$ (very hot annulus of moderate thickness):

$$
\begin{gathered}
I_{B}(\omega) \approx \begin{cases}\pi\left[B_{B}-B_{A}\right] & \omega<\omega_{B}^{\star}{ }_{B}, \\
\pi \alpha_{B} 2 a\left[B_{B}-B_{A}\right], & \omega_{B}^{\star}<\omega<\omega_{A}^{\star} \\
\pi \alpha_{B} 2 a B_{B}<\omega\end{cases} \\
I_{A}(\omega) \approx \begin{cases}\pi\left[B_{A}(1-\Gamma)+B_{A}-B_{B}\right] & , \omega<\omega_{B}^{\star} \\
\pi\left[B_{A}(1-\Gamma) *\left(B_{A}-B_{B}\right) 2 \alpha_{B} a\right], & , \omega_{B}^{\star}<\omega<\omega_{A}^{*} \\
\pi \alpha_{A} 2 \delta_{A} B & , \omega_{A}^{\star}<\omega .\end{cases}
\end{gathered}
$$

For Case II where $\omega_{A}^{\star}<\omega_{B}^{\star}$ (very thin annulus):

$$
I_{B}(\omega)_{B} \approx \begin{cases}\pi\left[B_{B}-B_{A}\right], & \omega<(1)_{A}^{\star} \\ \pi B_{B}(1-\Gamma), & \omega_{A}^{\star}<\omega<\omega_{B}^{\star} \\ \pi \alpha_{B} 2 a B_{B} & , \quad \omega_{B}^{\star}<\omega\end{cases}
$$




$$
I_{A}(\omega) \approx \begin{cases}\pi\left[B_{A}(1-\Gamma)+B_{A}-B_{B}\right] & , \omega<\omega_{A}^{\star} \\ \pi\left[B_{A} 2 \alpha_{A} \delta_{A}-B_{B}(1+\Gamma) \alpha_{A} \delta_{A}\right] & , \omega_{A}^{\star}<\omega<\omega_{B}^{\star} \\ \pi B_{A} 2 \alpha_{A} \delta_{A} & , \omega_{B}^{\star}<\omega .\end{cases}
$$

For a transparent plasma $\left(\omega^{*}<\omega\right)$, the formulas in Ref. 2 are extended to get $\alpha_{j}(\omega) L_{j} \approx p_{j} \exp \left[-q_{j} \omega / \omega_{c}\right]$ with

$$
\begin{aligned}
& p_{j}=\Lambda_{j}\left\{0.50\left(T_{j} / 10\right)^{-0.76}+0.003\right\} \\
& q_{j}=3.33\left(T_{j} / 10\right)^{-0.29}-1.13 \\
& \Lambda_{j}-\omega_{p j} \angle L_{j} / \omega_{C} \\
& L_{j}= \begin{cases}2 a, \text { buīk piàsimia } \\
\delta_{A}, \text { annulus }\end{cases}
\end{aligned}
$$

where $T$ is in keV.

For the EBT reactor reference parameters, if the bulk average plasma and cyclotron frequencies and an average radius $a=0.88 \mathrm{~m}$ are used to compute $\Lambda_{B}$, then one finds from Ref. 2 that the bulk plasma black body spectrum cuts off around $\omega_{B}^{\star} \approx 5 \omega_{C B}$. The annulus is harder to treat since its theoretical beta and thickness are presently unknown. However, for a variety of possible upper limits on these parameters one finds the product $\omega_{D A}{ }^{2} L_{A} \lesssim 1.4 \times 10^{21} \mathrm{~m} / \mathrm{sec}^{2},\left(\mathrm{e} . \mathrm{g} ., B_{A}=.08, \delta_{A}=1.8 \mathrm{~cm}\right)$, and in all cases $\Lambda_{A} \approx 10$. Consequentily, for a 100-keV annulus $\omega_{A}^{\star} \approx \omega_{C A}$. That is, the annulus is so thin and tenuous that it never radiates as a black body.

With these cutoff frequencies the cyclotion radiation spectra of the bulk and ammular plasmas are plotted in Figs. $\mathrm{C}-2$ and $\mathrm{C}-3$, using $\mathrm{I}(\omega)$ for Case II. (The dashed curves are radiation without reflecting walls; note that $\Gamma=0.9$ substantially reduces bulk plasma losses.) There is so much energy loss from the higher harmonics that spectral modifications near $\omega_{c}$ and $2 \omega_{c}$ should not significantly change the total cyclotron power loss. Thus, use of $n_{r}=1$ in the equation of transfer yields reasonable results even though the plasma density may cut of the lowest harmonics.

The average cyclotron power density loss is the quantity of interest. It is obtained by integrating $I(\omega)$ over all frequencies, multiplying this flux by plasma surface area, and dividing by plasma volume:

$$
\begin{aligned}
P_{C B} & =\frac{2}{a} f_{n}^{\infty} d \omega I_{B}(\omega) \\
& =\frac{4.5 \times 10^{-35}}{a} T_{B} \omega_{C B} 3\left\{(1-\Gamma) \frac{\left(m^{\star}{ }^{3}-1\right)}{3}+\frac{p_{B}}{q_{B}^{3}} \Gamma\left(3, q_{B} m_{B}^{*}\right)\right\} ; \\
P_{C A} & =\delta_{A}^{-1} \delta_{0}^{\infty} d \omega I_{A}(\omega) \\
& =\delta_{A}^{-1} 4.5 \times 10^{-35} T_{A}{ }^{\omega}{ }_{C A}{ }^{3} \frac{p_{A}}{q_{A}^{3}}\left\{\left[1-\frac{T_{B}}{T_{A}} \frac{(1+\Gamma)}{2}\right] \Gamma(3, x) \mid{ }_{q_{A} m^{*}}^{q_{A}}+\Gamma\left(e, q_{A} m^{\star}{ }_{B}\right)\right\}
\end{aligned}
$$




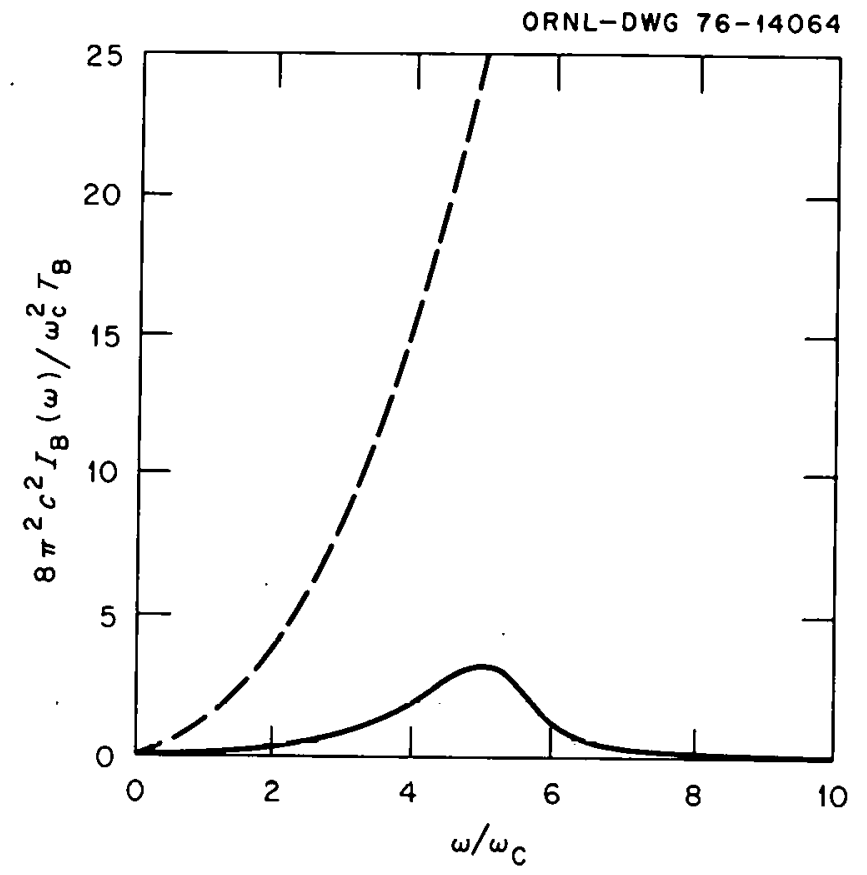

Fig. C-2. Cyclotron radiation spectrum of bulk plasma (solid); $n_{e}=1.2 \times 10^{20} \mathrm{~m}^{-3}, T_{e}=15 \mathrm{keV}$, $B=2.5 \mathrm{~T}, a=0.88 \mathrm{~m}, \Gamma=0.9$. Dashed curve is black body portion of spectrum in absence of reflecting walls.

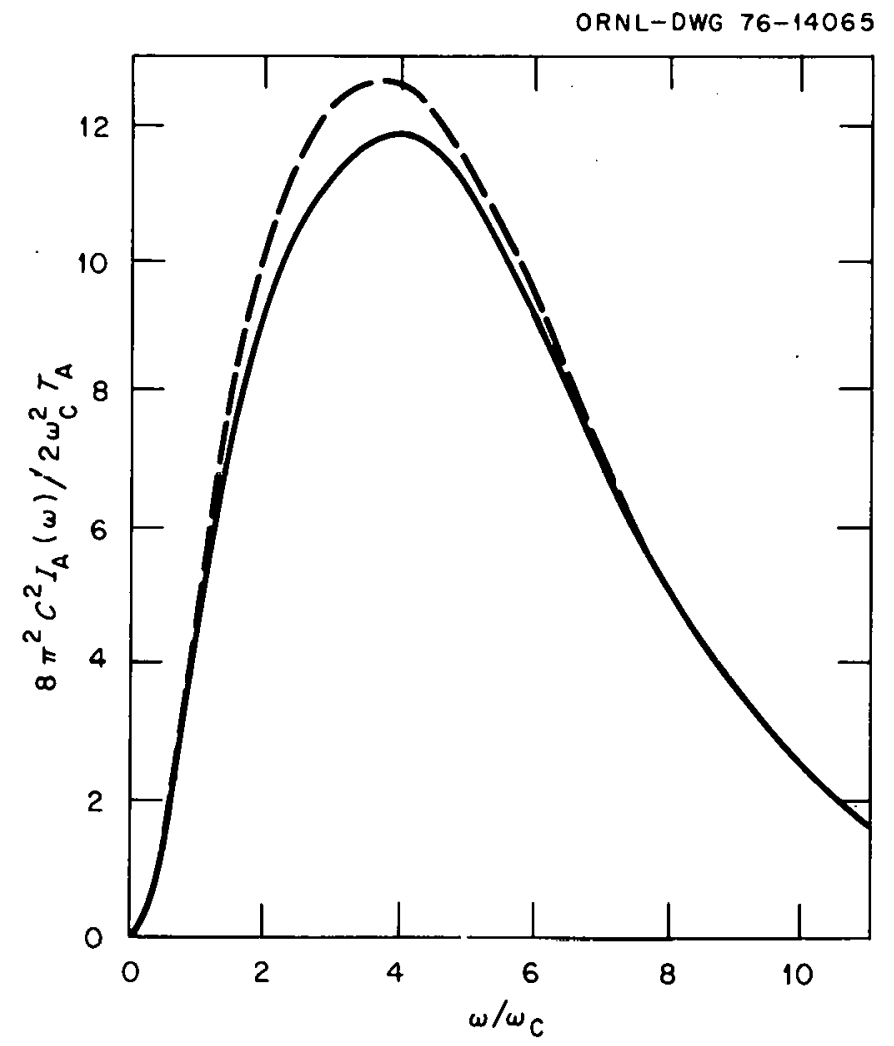

Fig. C-3. Cyclotron radiation spectrum of typical annulus (solid); $\mathrm{n}_{\mathrm{e}}=1.2 \times 10^{19} \mathrm{~m}^{-3}, \mathrm{~T}_{\mathrm{e}}=100 \mathrm{keV}$, $B-2.5 \mathrm{~T}, \delta_{\mathrm{A}}=1.8 \mathrm{~cm}, \Gamma=0.9$. Uashed curve is the same spectrum in the absence of reflecting walls. 
Here $\Gamma(a, b)$ is the incomplete gamma function, $\omega^{\star} \equiv m^{\star} \omega_{c}, m^{\star}{ }_{A} \equiv 1, T$ is in keV, lengths are in meters, and $P_{C}$ is in watts $/ \mathrm{m}^{3}$. (Note that in the unlikely event that $\Gamma \gtrsim 0.99$ the formula for $P_{C B}$ becomes a function of the annulus length.)

One question briefly considered in the study was the effect of the nonuniform magnetic field on cyclotron radiation. Since $\nabla B \neq 0$, the electron orbits are not simple gyrations and the single particle emission spectrum ought to be appropriately modified. However, in a reactor the variation of $B(r)$ is so small over a gyroradius that for these high energy electrons the usual relativistic effects dominate and Eqs. (3) and (4) seem reasonable. Another caution on the results is that the total plasma beta should include the alpha particle pressure. Consequently, since the very heavy alpha particles have negligible cyclotron losses, the $\Lambda_{j}$ parameter should be reduced from the $n_{\alpha}=0$ case and the cyclotron losses would be reduced. For reasonable alpha particle densities, e.g., $n_{\dot{\alpha}}<0.03 n_{j}$, this also turns out to be a small effect.

As an application of the formulae, the bulk plasma losses would be computed in the absence of any reflection at the first wall, $r=0$. For $T_{B}=15 \mathrm{keV}, n=1.2 \times 10^{20} \mathrm{~m}^{-3}, \bar{B}=3.5 \mathrm{~T}$, and $a=0.88 \mathrm{~m}$, Eq. (3) gives $P_{C B}=8.5 \mathrm{kw} / \mathrm{m}^{3}$. An alternate method of calculating this, originally due to Trubnikov ${ }^{3}$ and later published by Rose and Clark, ${ }^{4}$ reduces the sum of the cyclotron emission of all the individual electrons, $w_{C}$, by a factor $K$. For the reference EBTR design $\omega_{C}=6.2 \times 10^{-17} B^{2} n T \times$ $\left[1+\frac{T}{204}\right]=1.47 \mathrm{MW} / \mathrm{m}^{3}$, and $K=0.006$, so $P_{C B}=8.8 \mathrm{kw} / \mathrm{m}^{3}$. There seems to be reasonably close agreement between Eq. (3) and Trubnikov's calculations for this case, and the result is encouraging since a radiation loss this small is less than one percent of the thermonuclear power density produced. Reflecting walls reduce the power loss even more, as seen in Fig. $\mathrm{C}-2$. For $\Gamma=0.9$ one obtains $P_{C B}=1.1 \mathrm{kw} / \mathrm{m}^{3}$. (Trubnikov's method is useful only for $\Gamma=0$.) It can be concluded that bulk plasma cyclotron losses are negligible during the steady state operation of the reference EBT reactor.

Equation (4) is useful for estimating the microwave power required to sustain the annulus. In steady state the annulus power balance (point model) is

$$
r_{\mu}=\Gamma_{\tau A} \mid r_{B r A} \perp r_{C A},
$$

where $P_{\tau A}=\frac{3}{2} \frac{{ }^{\prime} A T_{A}}{\tau_{A}}$ represents thermal conduction and convection, $P_{B r A}=3.2 \times 10^{-37} n_{A}{ }^{2} T_{A}{ }^{1 / 2}$ is bremsstrahlung from the annulus, and $P_{C A}$ is given by Eq. (4). For $T_{A} \gg T_{B}, P_{C A}$ is only weakly dependent on wall reflectivity, so that $\Gamma=0.9$ should yield representative results. Then, with $m_{A} \approx \approx 1$, we find $P_{C A}$ and $P_{T A}$ are roughty proportional to $T_{A}$, while $P_{D r A} \propto T_{A}^{\frac{1}{2}}$. For the sake of comparison one might take $T_{A}=100 \mathrm{keV}$ and $T_{B}=15 \mathrm{keV}$, so

$$
\begin{aligned}
& P_{B r A}-3.2 \times 10^{-36} n_{A}^{2} \\
& P_{\tau A}=2.4 \times 10^{-14} n_{A} / \tau_{A} \\
& P_{C A}=3.5 \times 10^{3} P_{A} / \hat{\delta}_{A} .
\end{aligned}
$$

The power lost from the annulus in each sector of the torus is found by multiplying these power densities by the annulus volume, $2 \pi a L_{A} \delta_{A}$. For this example take $a=1.0 \mathrm{~m}, L_{A}=1.96 \mathrm{~m}$, and $\delta_{A}=0.01 \mathrm{~m}$. Finally, since $\mathrm{p}_{A} \propto \mathrm{n}_{\mathrm{A}}$, all three losses are sensitive to annular electron density, or, equivalently, to annular beta. Taking $\tau_{A}=1.0 \mathrm{sec}$ as an example, one is able to construct Table $C-1$ for three postulated values of $B_{A}$. 
TABLE $C-1$

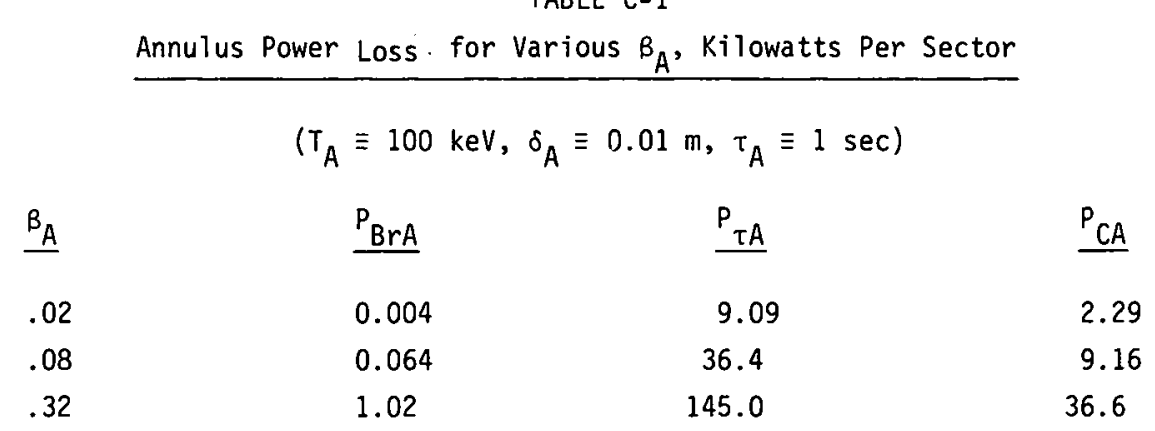

In all cases, it is found that the bremsstrahlung is insignificant compared to cyclotron radiation from the annulus, and the cyclotron losses are quite tolerable when compared with the typical thermonuclear power production ( $₹ 10^{2} \mathrm{MW} /$ sector). Very roughly speaking one can see from Eq. (4) that $P_{C A} \propto T_{A} P_{A} \propto T_{A} n_{A} \delta_{A} \propto \beta_{A}$ and from Table $C-1$ one gets the scaling law: $P_{C A} \approx 100 B_{A} \delta_{A} \mathrm{kw} / \mathrm{sector}$ $\left(\delta_{A}\right.$ in $\left.\mathrm{cm}\right)$. However, the most important observation from Table $C-1$ is that $P_{\tau A} \gg P_{C A}$, even for a long one second energy containment time for the annulus. For a thin annulus (of the order of a few centimeters) much shorter containment times are conceivable, and, since $P_{\tau \dot{A}} \propto{ }^{\tau}{ }_{A}^{-1}$, classical or neoclassical energy transport may determine the microwave power required to sustain steady state annuli.

\section{REFERENCES}

1. W. E. Drummond and M. N. Rosenbluth, Phys. Fluids $6,276 \cdot(1963)$.

2. J. L. Hirshfield, D. E. Baldwin, and S. C. Brown, Phys. Fluids $\underline{4}, 198$ (1961).

3. B. A. Trubnikov and V. S. Kudryavtsev, Proc. 2nd U.N. Conf. on Peaceful Uses of Atomic Energy, Vol. 31 , p. 40 (1958).

4. D. J. Rose and M. Clark, Jr., Ch. 11 in Plasmas and Controlled Fusion, MIT, Cambridge, 1965. 


\section{INTRODUCTION}

The present state of knowledge requires that the design of fusion reactors be based on scaling laws which have not been fully verified by experiments. Various scaling laws are assumed in modeling the plasma; this leads to differences in the results, which depend on the details of the assumptions themselves.

Two of the important parameters in designing and determining the feasibility of an EBT reactor are the plasma particle and energy lifetimes. The key question then is which of the various theories that predict these lifetimes is most appropriate. During start-up the plasma temperature and density change through many orders of magnitude, so it seems evident that different transport models will apply at different times. Even in steady-state operation, different regimes of plasma will have different density and temperature profiles, so that different physical processes will dictate plasma behavior depending on the spatial location analyzed. Moreover, the uncertainties surrounding present-day transport theories have resulted in a large number of possible diffusion coefficients. Reactor modeling must be carried out using the theories which seem to be the most plausible ones. Within neoclassical theory, effects due to radial electric fields, finite beta, non-Maxwellian particle distributions, microwave fields, and the presence of multiple species act to compound the difficulty. Even within classical theory, it is difficult to self-consistently and separately characterize the processes of heat conduction and convection in an anisotropic plasma.

In view of the uncertainties in the theoretical coefficients and the present lack of experimental evidence in this area, it seems reasonable to model the plasma using both classical and neoclassical theory. Flexibility will be retained in the simulation models to permit updated data to be incorporated as they become available. The particle and energy confinement times have been chosen consistently with reactor design and current understanding of the plasma physics involved. The absolute numerical values of confinement times and limiting beta are important design determinants and must be approached if the EBT concept as developed here is to prove feasible. The detailed scaling of these parameters is less critical; this is the same approach which is used in tokamak reactor studies.

The following studies demonstrate the sensitivity of the EBT reactor design features to the scaling laws assumed. The models are suitable for simulating the energr and particle balances in a bumpy torus plasma.

\section{PHYSICS MODEL}

As a first step in assessing the energy balance in an EBT reactor plasma, a simple model has been analyzed in which the bulk plasma is characterized by simple energy and particle containment times with appropriate density and temperature scaling. This model is consistent with the physics model described in Appendix B: a toroidal core with nearly uniform density and temperature within the plasma radius determined by the stabilizing electron annuli. The resulting particle and energy balance equations are:

$$
\frac{d n_{D}}{d t}=n_{e} n_{O_{D}}<\sigma v{ }_{i o n}+S_{D C O L D}+S_{D B E A M}(1-f)-n_{D} n_{T}<\sigma v>{ }_{D T}-\frac{n_{D}}{\tau_{p, D}}
$$




$$
\begin{aligned}
& \left.\frac{d n_{T}}{d t}=n_{e} n_{0_{T}}<\sigma v\right\rangle_{\text {ion }}+S_{\text {TCOLD }}-S_{\text {DBEAM }} f-n_{D} n_{T}<\sigma v>{ }_{D T}-\frac{n_{T}}{\tau_{p, T}} \\
& \left.\frac{d n_{\alpha}}{d t}=S_{\text {DBEAM }} f+n_{D} n_{T}<\sigma V\right\rangle_{D T}-\frac{n_{\alpha}}{\tau_{p, \alpha}} \\
& n_{e}=n_{D}+n_{T}+2 n_{\alpha} \\
& n_{0}=n_{O_{D}}+n_{O_{T}} \\
& \frac{d}{d t}\left(\frac{3}{2} n_{e}{ }_{e}\right)=S_{D B E A M}\left[U_{D B E A M} G_{e}+f U_{\alpha} f_{\alpha e}\right]+P_{\mu} \\
& +n_{D} n_{T}<\sigma V>D T U_{\alpha} f_{\alpha e}-P_{R A D} \\
& -1.5 \times 10^{-19} \frac{n_{e}}{T_{e}^{3 / 2}} \cdot \ln \Lambda\left(T_{e}-T_{i}\right)\left(\frac{n_{D}}{2}+\frac{{ }^{n_{T}}}{3}\right) \\
& -\frac{3}{2} \frac{n_{e} e^{T}}{\tau_{E, e}} \\
& \frac{d}{d t}\left(\frac{3}{2} n_{i} T_{i}\right)=S_{\text {DBEAM }}\left[U_{\text {DBEAM }} G_{i}+f U_{\alpha} f_{\alpha i}\right] \\
& +n_{D} n_{T}<\sigma V>{ }_{D T} U_{\alpha} f_{\alpha i}-\frac{3}{2} \frac{n_{j} T_{i}}{T_{E, i}} \\
& +1.5 \times 10^{-19} \frac{n_{e}}{T_{e}^{3 / 2}} \ln \Lambda\left(T_{e}-T_{i}\right)\left(\frac{{ }^{n}}{2}+\frac{{ }^{n} T}{3}\right)
\end{aligned}
$$

The parameters used in the equations are defined as follows:

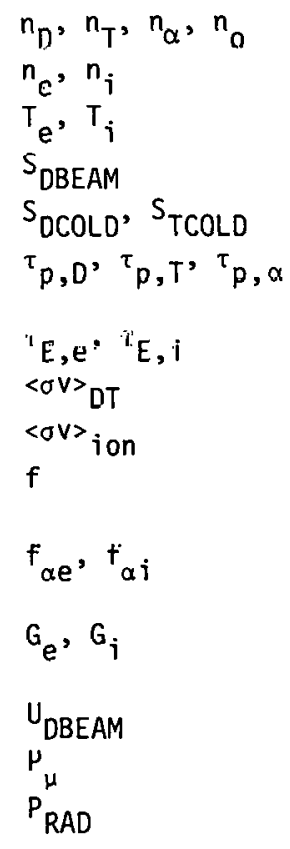

Average deuterium, tritium, alpha, and neutral densities. Average electron and ion densities. Average electron and ion temperatures. Deuteriun source from fast ions. Deuterium, tritium cold fueling rate. Particle confinement times of deuterium, tritium, and alphas.

Energy confinement times of slectrons and ions,

D-T fusion reaction rate.

Ionization rate.

Fraction of injected deuterium beam which undergoes supradherilld 7 rusiun.

Fraction of fusiun pruducud alplia enerijy delivered ton the electrons and ions.

Fraction of injected deuterium beam power delivered to the electrons and ions.

Neutra1 beam injection energy.

Micruwave puwer delivered to background electrons.

Total power loss from radiation (bremsstrahlung, cyclotron, etc.).

A brief description of the key terms in the above equations is given below. 


\subsection{Containment Times}

The particle and energy containment times are critical in determining the detailed energy balance for a system. Since at present the scaling laws appropriate to large, hot plasmas in the EBT configuration are not known, theoretical and empirical estimates have been used. From appropriate transport coefficients, particle confinement times for the $j$ th thermal specie are given by

$$
\tau_{p, j}= \begin{cases}\text { Classical: } & c_{1} n_{j}^{-1} T_{j}^{T / 2} \\ \text { Neoclassical: } & \frac{c_{2}}{\nu_{j}}\left(1+\frac{\nu_{j}^{2}}{\Omega_{j}^{2}}\right) \\ \text { others: } & \end{cases}
$$

where $C_{j}$ is a constant which is adjusted so that $\tau_{p, j}$ has a desired value at the steady-state operating conditions. Also,

$$
\begin{aligned}
& v_{j}=3.8 \times 10^{-17} \frac{Z_{j}^{2} n_{j}}{\sqrt{A_{j}} T_{i}^{3 / 2}} \\
& \Omega_{j}=\frac{10^{3}}{Z_{j} B R_{c}^{a}}\left[1+Z_{j} n\right] T_{i} \text { with } n=10^{-3} \frac{E_{r} R_{c}}{T_{i}} \\
& c_{2}=\frac{3}{4} \frac{R_{T}}{R_{c}}\left[\left(1+z_{j} n\right)\right]^{2} .
\end{aligned}
$$

$R_{T}$ and $R_{C}$ are the toroidal and mirror radii of curvature and $E_{r}$ is the radial electric field. From quási-neutrality

where

$$
\tau_{p, e}=\tau_{p, j},
$$

$$
\tau_{p, i}^{-1} \cong \frac{n_{D}}{n_{i}} \tau_{p, D}^{-1}+\frac{n_{T}}{n_{i}} \tau_{p, \tau}^{-1}
$$

The electron and ion energy containment times, $\tau_{E, e}$ and $\tau_{E, i}$, may be different. For electrons

$$
{ }^{\tau} E, e \approx C_{3} \tau_{p, i}
$$

and for ions

$$
\tau_{E, i}^{-1}=c_{3}^{-1} \tau_{p, i}^{-1}+\tau_{c x}^{-1}
$$

$c_{3}$ is a constant and $\tau_{c x}$ is the time for charge exchange between hot ions and cold neutral atoms.

\subsection{Radiation Losses}

$$
P_{\text {RAD }}=P_{\text {BREM }}+P_{\text {LINE }}+P_{\text {RECOMBINATION }}+P_{\text {CYCLOTRON }}
$$

a. $\mathrm{P}_{\text {BREM }}+\mathrm{P}_{\text {LINE }}+\mathrm{P}_{\text {RECOMBINATION }}$

$$
\begin{gathered}
\approx n_{e} z_{e f f}\left[3 \times 10^{-21} \mathrm{~T}_{e}^{1 / 2}+1.14 \times 10^{-22} \mathrm{~T}_{\mathrm{e}}^{-1 / 2}\right. \\
\left.+2.58 \times 10^{-24} \mathrm{~T}_{\mathrm{e}}^{-3 / 2}\right] \frac{\mathrm{keV}}{\mathrm{m}^{3}-\mathrm{s}}
\end{gathered}
$$

b. Perclotron is discussed in detail in Appendix $C$. 


\subsection{Alpha Energy Deposition}

The fraction of the alpha energy produced by fusion which is delivered to the electrons, $f_{\alpha e}$, is

$$
f_{\alpha e} \approx 1-\frac{2}{x}\left[\frac{1}{6} \ln \left(\frac{1-\sqrt{x}+x}{(1+\sqrt{x})^{2}}\right)+\frac{1}{\sqrt{3}} \tan ^{-1}\left(\frac{2 \sqrt{x}-1}{\sqrt{3}}\right)+.3023\right] \text {, }
$$

where $x \equiv U_{\alpha} / U_{\alpha c r}$, and $U_{\alpha c r}$ is the critical energy, i.e., the energy at which alpha particle energy is transferred to the electrons and ions at equal rates. The fraction delivered to the ions, $f_{\alpha i}$, is $\left(1-f_{\alpha e}\right)$.

\subsection{Neutral Beam Injection Heating}

The energy delivered to the background plasma electrons and ions from injection of fast deuterium atoms is given by the functions $G_{e}$ and $G_{i} \cdot{ }^{1} \quad G_{e}$ and $G_{i}$ can be found, neglecting charge exchange, by using Eq. (13) for $f_{\alpha_{C}}$ and letting $x=U_{D B E N M} / U_{c^{p}}$, where $U_{c_{F}}$ is the critịcal enerqy as defined above but for the fast injected ions.

When charge exchange is included, an approximate fit to the $G_{e}, G_{i}$ curves yields

$$
\begin{gathered}
G_{i} \approx 0.5 \exp \left[-0.1 \frac{U_{\text {DBEAM }}}{U_{C r}}\left(1+0.5 \frac{\tau_{s}}{\tau_{c x}}\right)\right] \\
+0.5 \exp \left[-0.6 \frac{U_{\text {DBEAM }}}{U_{c r}}\left(1+0.5 \frac{{ }^{\tau} s}{\tau_{c x}}\right)\right] \\
G_{e}=1-G_{i}-G_{c x},
\end{gathered}
$$

where

$$
\begin{aligned}
& G_{c x} \approx 1-\exp \left[\cdot 0.121\left(\begin{array}{c}
\tau_{s} \\
\tau_{r \gamma}
\end{array}\right) \begin{array}{c}
U_{\text {DBEAM }} \\
U_{r r}
\end{array}\right], \underset{U_{r r}}{U_{\text {DBEAM }}} \leq 1 \\
& \approx 1-\exp \left[-0.121\left(\frac{{ }^{\tau} s}{\tau_{c x}}\right)\left(\frac{U_{\text {DBEAM }}}{U_{G r}}\right)^{T / 2}\right], \frac{U_{\text {DBEAM }}}{U_{c r}}>1 .
\end{aligned}
$$

$\tau_{s}$ is the Spitzer ion-electron momentum exchange time.

\subsection{Further Comments on the Model}

Two distinct models have been studied. The critical assumptions and approximations included in these models are described below.

a. Classical scaling: The temperature and density behavior of the themalized electrons, deuterons, and tritons have been examined by assuming classical scaling for both the particle and energy confinement times. In addition to the classical assumption: ( $i$ ) The energy confinement time is taken to be 2.5 seconds at the reference operating point (i.e., $n \tau \simeq 3 \times 10^{20} \mathrm{sec} / \mathrm{m}^{3}$ ), and the ratio of particle to energy confinement time is assumed to be three. (ii) The effects of a background neutral density are neglected. (iii) Thermal alpha particles are assumed to diffuse out of the plasma at approximately the same rate as the other ion species, which results in a small thermal alpha particle population. [Eq. (3) is not used.] (iv) The plasma beta limit is chosen consistently with the limiting beta stability model of Appendix $B$. When the value is exceeded, the particle confinement is modified so as to maintain constant plasma energy density. For most cases considered, including the start-up run described below, the limiting energy density was given by

$$
n_{i} T_{i}+n_{e} T_{e} \leq B_{1 i m} \frac{B^{2}}{2 \mu_{0}}=7.2 \times 10^{5} \mathrm{~J} / \mathrm{m}^{3} \text {. }
$$


When the beta limit is exceeded, the particle loss rate is assumed to increase; as a result, the plasma reactivity decreases and limits the thermal excursion which otherwise occurs due to the variation of confinement time with temperature. (v) $z_{\text {eff }}$ is taken as unity. With divertors, this assumption is probably valid. Z eff considerably larger than unity would have a deleterious effect on both start-up and steady-state requirements. (vi) Neutral beam injection heating is included and suprathermal fusion due to beam-plasma interactions is neglected. (vii) The electron annulus is physically separated from the bulk plasma and power transfer between the electron ring and the bulk plasma is included in the synchrotron radiation term.

b. Neoclassical scaling: In the present EBT device, plasma transport seems to be governed largely by neoclassical processes. Analyses leading to this conclusion include a treatment of the ambipolar electric field and the presence of a non-Maxwellian electron population. The containment times are derived from the appropriate transport coefficients originally calculated by Kovrizhnykh ${ }^{2}$ (for the case of a strong radial electric field) and modified by Harris and Spong ${ }^{3}$ to include arbitrary values of the ambipolar electric field. In addition to the neoclassical assumption: (i) Since the transport calculations of Harris and Spong are still being developed and will include the effects of finite plasma pressure, the microwave power and radial electric field presently are treated as parameters rather than self-consistently. This approach permits many unknowns to be lumped together in these parameters. (ii) The rate of energy transport includes a contribution from thermal conductivity. (iii) Charge exchange losses are incorporated into the model. (iv) Thermat excursions to high temperatures also have been observed as in the classical model but here no beta-limiting mechanisms have been included in the model. Again the thermal excursion is due to the favorable scaling assumed and is identical to the results obtained a few years ago in low-beta systems studied where neoclassical scaling was assumed.

In the next section, the results for EBT start-up will be discussed. These results are based on the classical model.

\section{EBT START-UP}

It is of interest to determine the characteristics of the neutral beam system which can be used to start up and ignite the EBT reaclur plasma. Also, a start-up scenario refers to a procedure for systematically controlling the reactor plasma parameters during the heating phase and to the achievement of the conditions necessary for the steady-state, self-sustaining operating point, For the purpuses of this study, it will be assumed that the beam power is held at a constant value until the steady state is reached, at which point the beam is terminated. The beam power used is $200 \mathrm{MW}$ and the initial beam energy is $150 \mathrm{keV}$. Both of these values are consistent with the recognized objectives of long-range beam development programs. The plasma density is built up and maintained by injection of cold neutral particles at an externally deterwined rate (e.g., some form of pellet injection). The contribution to particle input from the neutral beams is much smaller than the required fueling rate. The cold fueling rate and, therefore, density control will influence the plasma behavior. It may be possible to use injection and fueling to reach steady state by one of the following apprnarhes

1. A. Initially maintain a fixed density and raise the temperature to the steady-state value. Then,

B. maintain the temperature constant and raise the density to the steady-state value.

2. A. Increase the temperature (or density) to an intermediate value, while the density (or temperature) remains fixed. Then,

B. repeat $(A)$ with the roles of the two quantities reversed, and

C. alternate $(A)$ and $(B)$ until the steady-state plasma parameters are attained. 
3. Increase the temperature and density simultaneously.

Note that reversing the roles of $(A)$ and $(B)$ in Case 1 will not permit the desired steady-state conditions to be reached. This is due to the fact that with a density of $\mathrm{n}_{\mathrm{e}} \sim 1.5 \times 10^{20} \mathrm{~m}^{-3}$, the power loss rate will exceed at some intermediate time the power input as the temperature is increased.

As an illustration of a successful start-up approach, a version of Case 2 will be shown. Using the reference reactor parameters and the assumptions listed in Section $2.5(a)$, consider the following start-up scenario:

(1) From 0 to $t_{1}(\sim .5 \mathrm{~s})$ the cold fueling is very rapid, thus causing the density to increase to about one-half of its steady-state value. During this time the temperature increases a few keV.

(2) From $t_{1}$ to $t_{2}(\sim 2.6 \mathrm{~s})$ the cold fueling rate is adjusted so that the density is held constant. With a constant beam power, the plasma temperature increases. At $t_{2}$ the steadystate temperature is reached.

(3) From $t_{2}$ to $t_{3}(\sim 4 s)$ the cold fueling is readjusted. The fueling rate then causes the density to build up to the steady-state value without quenching the plasma (i.e., the steadystate ion temperature is maintained).

(4) At $t_{3}$ the beam is turned off and the fueling rate is set to a value to maintain a constant density. At this point, the temperature would continue to rise (due to the transport assumptions) unless there is some mechanism such as a beta-limit to increase losses and stabilize it.

The fueling rate and the resulting density time histories are shown in Fig. D-1 and the temperatures are given in Fig. D-2.

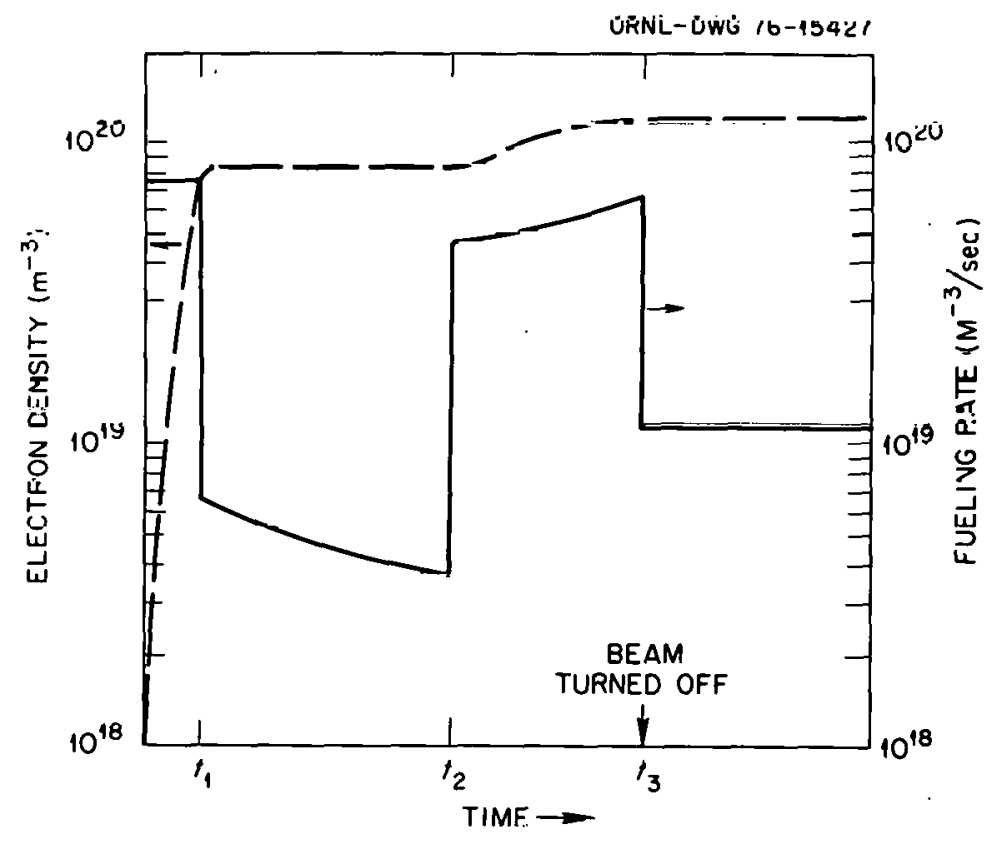

Fig. D-1. Fueling rate and density vs time during start-up.

There are a number of interesting observations that should be made with respect to the temperature profiles. 
At the beginning of the start up (0-0.5 s) the temperature rises rapidly. This is because the initial density is low, and only a low power input is needed to rapidly increase the average particle energy. (The temperature can be viewed as a measure of average particle energy.) Note that at low temperatures the beam heats the electrons preferentially.

In the final stage of the first start-up phase the density has increased to the point that transport losses are significant. As a result, the rapid temperature increase no longer occurs. Furthermore, the electron-ion equilibration becomes more pronounced due to the increased collision frequency at higher densities.

In the second stage of start up (0.5-2.6s) the temperatures of the electrons and ions are approximately equal and continue to increase. This result occurs since $n_{e} \sim 8 \times 10^{19} \mathrm{~m}^{-3}$ and coulomb collisions between electrons and ions transfer the input energy efficiently from the electrons (where it is deposited by the beams) to the ions.

In the third stage (2.6-4.0 s) the density is increased. This significantly increases the alpha power produced by the plasma. Since the alphas preferentially heat the electrons, the electron temperature increases modestly above the ion temperature.

Other considerations have been included in the numerical model that are not considered in the particular case illustrated here. For example, if the suprathermal alpha confinement time ( $\left.\tau_{p \alpha}\right)$ is small compared to the alpha slowing down time, $t_{2}$ and $t_{3}$ would increase. If $\tau_{p \alpha}$ becomes very small, perhaps due to instabilities, the possibility of achieving ignition at all would be questionable.

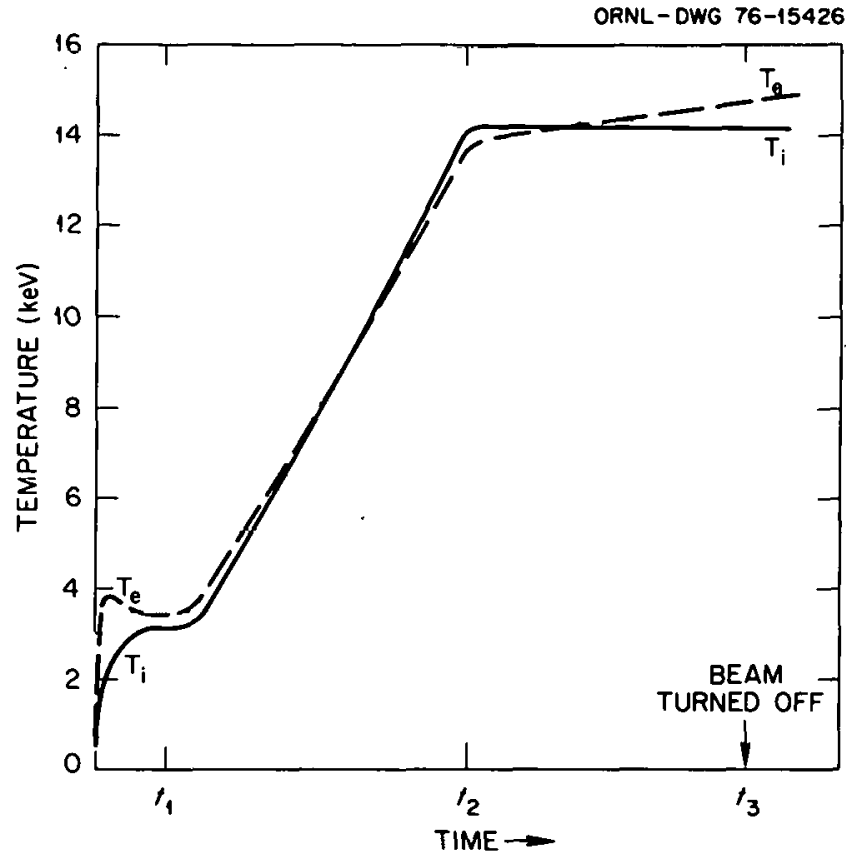

Fig. D-2. Temperature vs time

\section{REFERENCES}

1. J. D. Callen et al., Neutral Beam Injection Into Tokamaks, Proc. 5th International Conference on Plasma Physics and Controlled Núclear'Fusion Research, IAEA, VoT. I (1975) 645. 
2. L. M. Kovrizhnykh, Transport Phenomena in Toroidal Magnetic Systems, Zh. Eksp. Teor. Fiz. $\underline{56}, 877$ (1969), [Sov. Phys. JETP, 29, 475 (1969)].

3. E. G. Harris, Transport in Bumpy Torus (unpublished, 1976), D. A. Spong et al., The Neoclassical Transport in the EBT Device, paper presented at "Annual Meeting on Theoretical Aspects of Controlled Thermonuclear Fusion" Meeting, Madison, Wisconsin, April 7-9, 1976, and C. L. Hedrick et al., Theoretical Calculations of Transport and Scaling in the Elnı Bumpy Torus (EBT), Paper CN-35/D7, 6th International Conference on Plasma Physics and Controlled Nuclear Fusion Research, Berchtesgaden, FRG, October 6-13, 1976 (proceedings to be published). 


\section{INTRODUCTION}

The EBT reactor magnet system designs are described in this appendix. Section 2 deals with general scaling, Sect. 3 with conductor design, Sect. 4 with coil design, Sect. 5 with cryogenics, and Sect. 6 with protection. Two designs were carried out, one for a 48-coil reactor with a major radius of $60 \mathrm{~m}$ (EBTR-48) and one for a 24-coil reactor with a major radius of $24 \mathrm{~m}$ (EBTR-24). The latter design has since been modified to a $24-c 0 i l$ reactor with a 30 m major radius. Aside from the plasma constraints summarized in Table E-1, the main criterion was to design coils that could be built with existing technology or with technology which is the objective of existing development programs.

TABLE E-1

\section{Plasma Constraints on the EBTR Toroidal Magnets}

$\begin{array}{lcc} & \text { EBTR-48 } & \frac{\text { EB TR-24 }}{} \\ \text { Major radius (m) } & 60 & 24 \\ \text { Number of coils } & 48 & 24 \\ \text { Mirror ratio } & 1.8 & 1.5-1.7 \\ \text { Magnetic field (on axis) } & & \\ \quad \text { Throat (T) } & 4.5 & 4.5-4.9 \\ \quad \text { Mid-plane (T) } & 2.5 & 2.9 \\ \text { Plasma radius (m) } & 1 & 0.8 \\ \text { Cold zone (m) } & 0.2 & 0.2 \\ \text { Blanket and shield thickness }(m) & 1.75 & 1.75\end{array}$

A description of an alternate magnet design for the 48-coil reactor is given in Sect. 7 . This design is based on the high current density, forced-flow, bundle conductor concept. This approach requires more development in technology but holds great promise for the future.

The magnet design considerations are summarized in Sect. 8.

\section{MAGNFT ST.ALIING LAWS}

Independent of plasma physics considerations, the number of coils, the mirror ratio, the coil radius, and the reactor major radius of the EBT configuration can all be related by the geometry of the vacuum magnetic fields. Plasma physics considerations further relate the maximum and minimum allowahle mirror ratios to the aspect ratio, the requisite electron ring beta, the stable plasma volume, the magnetic axis shift, and similar quantities. The physics indicates that large aspect ratio systems are desirable, all else being equal, but present understanding of these systems is not yet sufficiently refined to yield quantitative data. However, a few relatively rigorous engineering constraints serve to fix the minor radius and the aspect ratio once the mirror ratio is fixed. The engineering constraints are more rigorous than those imposed by the physics; an optimized engineering design will almost certainly be in a regime that satisfies the physics requirements.

The mirror ratio can be related to the major radius $R$, the coil radius $d$, and the total number of coils $N$, by 


$$
M \simeq \frac{1+\frac{2 N d}{R} K_{1}\left(\frac{N d}{R}\right)}{1-\frac{2 N d}{R} K_{1}\left(\frac{N d}{R}\right)}
$$

where $\mathrm{K}_{1}$ is the modified Bessel function. The mirror ratio in EBT reactor systems will be in the range of 1.5-2.0 and the coil bore, set by shielding requirements, will not be much less than 3.0-3.5 $\mathrm{m}$. This expression relates the number of sectors in the system to the magnet aspect ratio, defined as $R / d$. Maximization of the stable plasma volume in the vacuum field of the bumpy torus suggests ${ }^{1}$

$$
M^{1 / 2} \simeq\left(N^{1 / 2}+1\right) /\left(N^{1 / 2}-1\right) .
$$

These "global" relations between the system parameters, along with reasonable values of current density in the coils, can be used to demonstrate that the number of coils will be large (at least 24 and nptimally perhaps twice that number) and that the coils will be widely spaced relative to the characteristic dimensions of the magnet winding. The wide spacing allows an optimization of the magnets which is relatively independent of the specific reactor system in which the magnets will be empluyed. One can, in effect, design a "standardized module" and, providing that the aspect ratio does not get too small, choose the final plant design on the basis of desired total power output. In this appendix, various design features are demonstrated, the sensitivity to different magnet design parameters is shown, and the advantages to be gained in adopting a somewhat more advanced conductor/cooling design are described. The object of this discussion is to show that although existing technology is nearly adequate to meet the relatively relaxed magnet requirements of an EBT reactor, there are further advantages to be gained if the technology is extrapolated reasunably.

The global parameters are inner bore radius, $R_{0}$; magnetic field intensity at the magnet throat, $B$; mirror ratio, $\mathrm{M}$; and total number of main coils in the reactor, N. The local parameters, those which affect only the magnet design, are the length of magnet, $L$; the average current density in the windings, $\langle\mathrm{J}\rangle$; and the internal magnet design. Both the radial thickness of the coils and the distance between magnets in the reactor are determined once the global and local parameters are specified. In the following discussion, a 48-coil EBT reactor (with no diverturs) is cunsidered.

As can be seen in Fig. $E-1$, the distance between magnets is only weakly dependent on $\langle j\rangle$ or magnet length for values of these parameters in the reactor regime. Thus, the global parameters can be set independently of the local magnet parameters, and the local parameters can then be optimized with regard to cost, cooling needs, and cryogenic stab111ty. Optinization of the current density distribution is drscussed in sect. 7.

The optimization of the magnet length is controlled by the following cunsiderations:

A) With all independent magnet parameters fixed except length, the cost of the coils can be minimized with respect to L (see Fig. E-2).

B) Heat deposition as the result of plasma operation makes a small magnet length more favorable, since in this case the magnet presents a smaller cross-sectional area to the pidasila.

c) The peak magnetic field at the inner surface of the windings is important. With the field in the magnet throat held constant, the peak magnetic field at the windings decreases as the coil length is increased. Thus, cryogenic stability tends to favor a longer magnet due to the magnetoresistance of the copper in the windings.

As can be seen from Figs. E-3 - E-6 the distance between magnets (for a fixed mirror ratio) and the mirror ratio (for a fixed magnet separation distance) are only weakly affected by the choice of the local parameters of the magnetic coils. They are more strongly dependent on the global parameters, such 
as the inner bore radius. The ratio of the total magnet cost to the plant thermal output is strongly dependent on both the global and local magnet parameters. The magnet cost figures are based on reasonable estimates of material and construction costs but should be used as comparative, rather than absolute, vaiues.

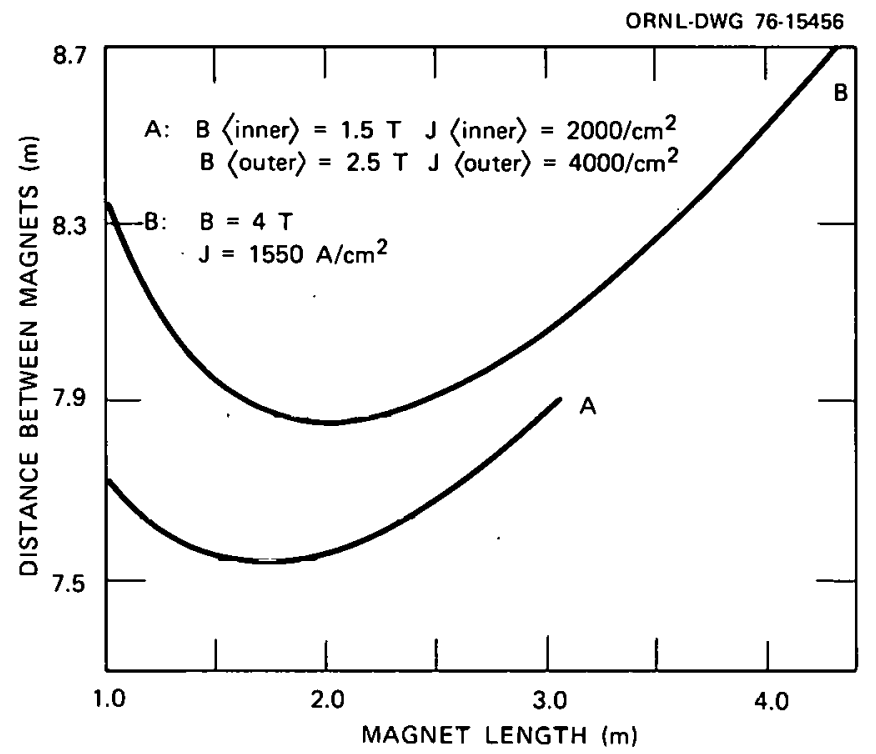

Fig. E-1. Distance between magnets vs magnet length for mirror ratio 1.8 .

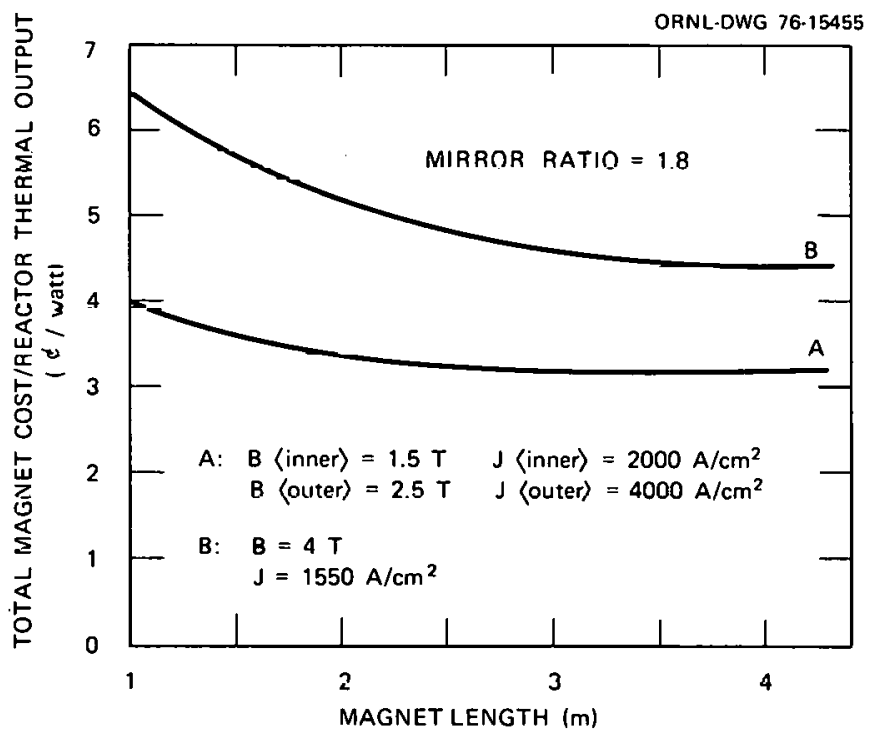

Fig. E-2. Optimization of magnet length with respect to magnet cost for a $4000 \mathrm{MW}(\mathrm{th})$ reactor. 


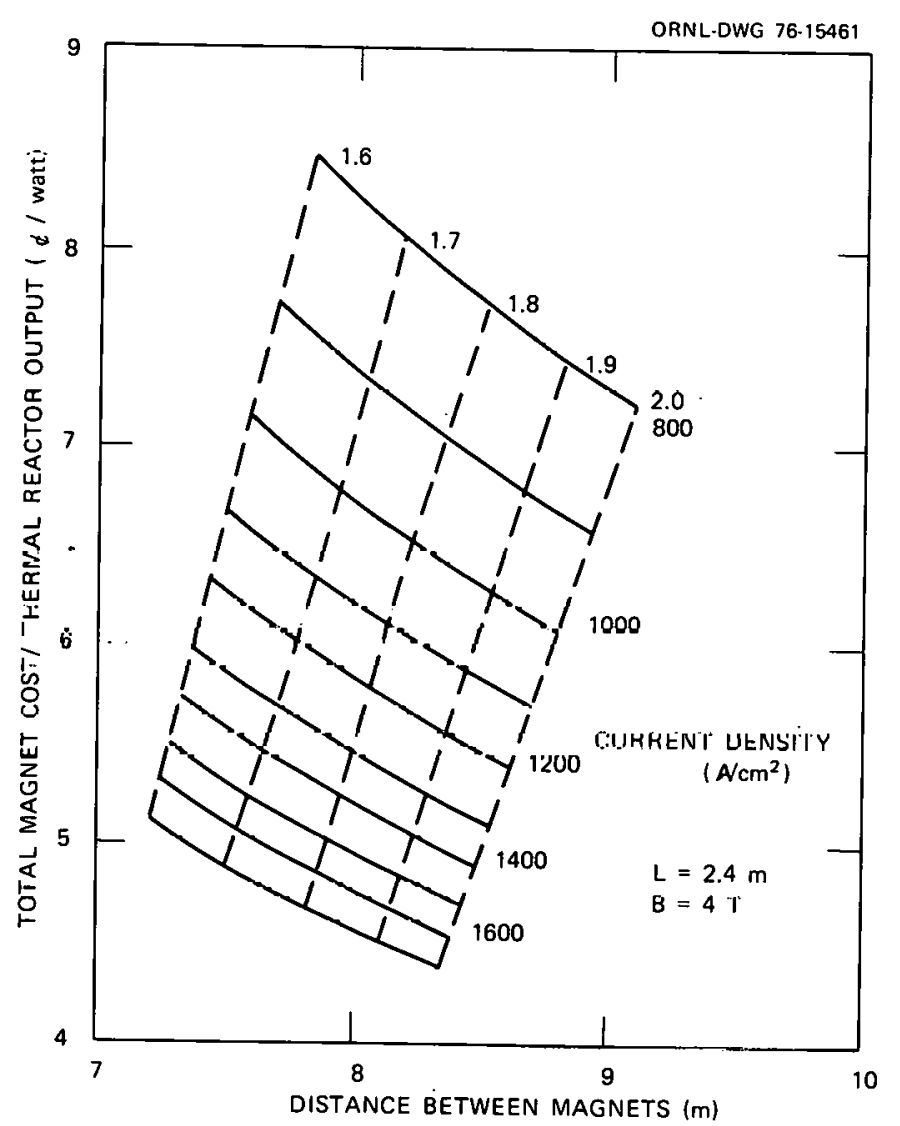

Fig. E-3. Magnet cost/thermal reactor output vs distance between magnets for various mirror ratios and current densities for the reference magnet design.

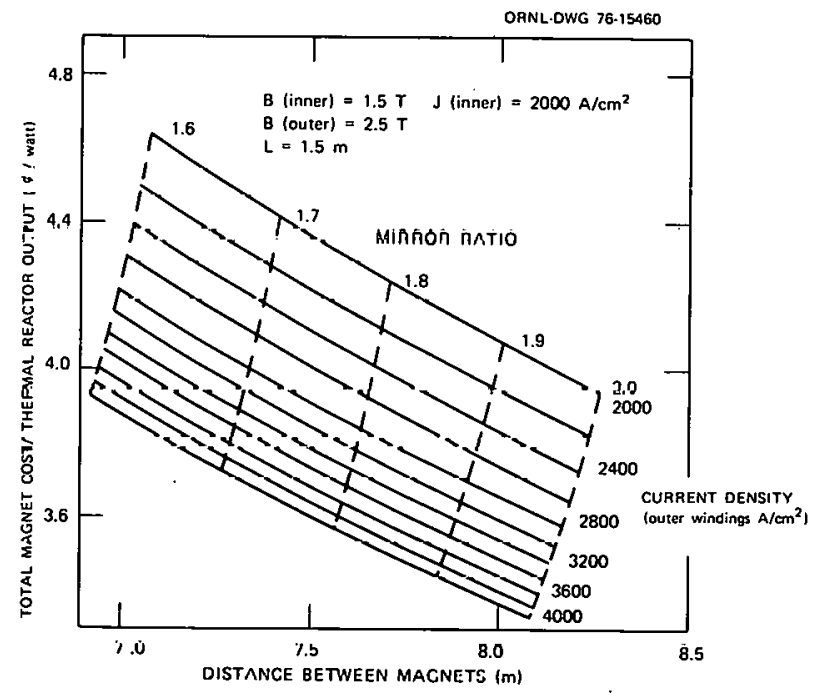

Fig. E-4. Magnet cost/thermal reactor output vs distance between magnets for various mirror ratios and current densities (at outer windings) for an alternate magnet design. 


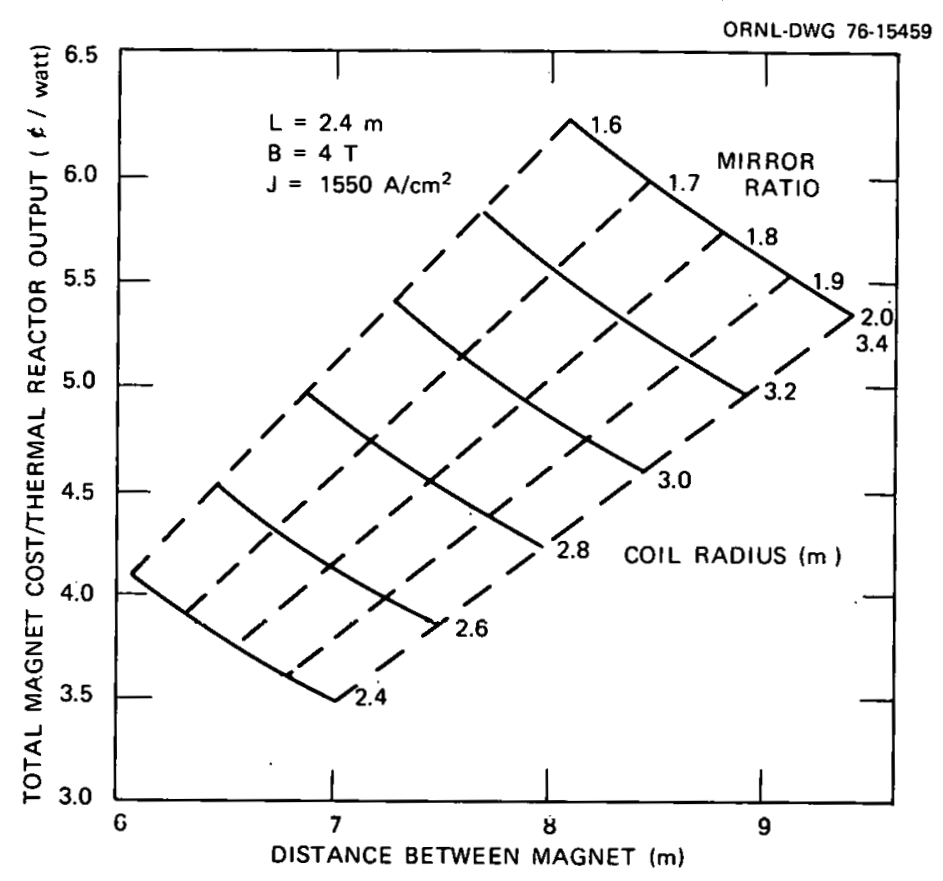

Fig. E-5. Magnet cost/thermal reactor output vs distance between magnets for various mirror ratios and coil radij for the reference magriel design.

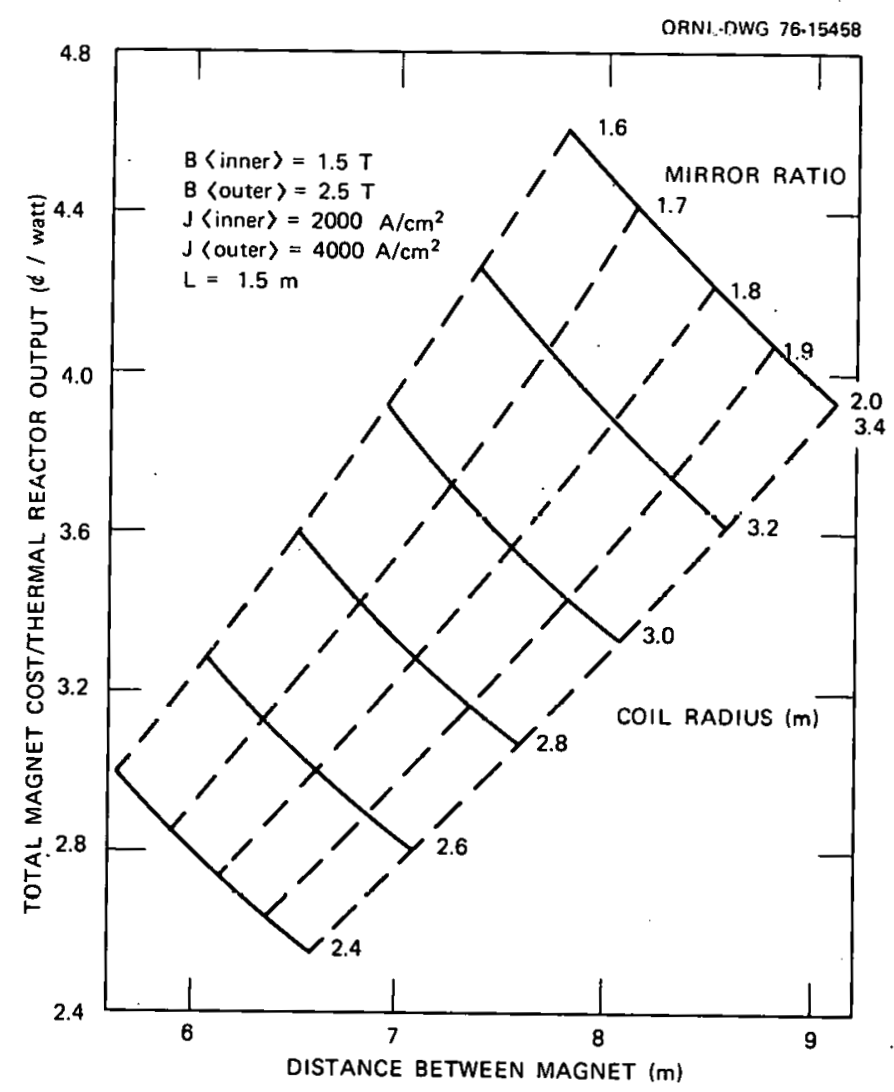

Fig. E-6. Magnet cost/thermal reactor output vs distance between magnets for various mirror ratios and coil radii for an alternate magnet design. 
Changing the current density or the length of the magnet will change the mirror ratio when the spacing of the magnets is fixed. As discussed before, however, the change in the mirror ratio will be small as the optimal design is varied, while the cost and weight of the magnet, in comparison, will rise rapidly. The internal design of the magnet does not dominate the relationship between mirror ratio and magnet separation distance and is an ineffective control variable.

\section{CONDUCTOR DESIGN}

\subsection{Design Criteria}

It is generally agreed that superconducting magnets are required for an economical fusion reactor. Reactor application also demands high reliability, which implies a fully cryostabilized conductor design. In contrast to the tokamak-type reactor, EBT has no pulsed magnetic fields. Hence the more exotic conductor designs, such as multi-component matrices (CuNi), very fine superconductor filament size $(<10 \mu \mathrm{m})$, hollow or forced-flow conductors, cabling and braiding of composite conductors, etc., are not required. These designs are aimed at reducing eddy current losses and/or increasing the heat transfer efficiency of the magnet coolant. They are more expensive than the conventional monolithic conductor and require considerable development.

With respect to superconducting materials, multifilamentary $\mathrm{Nb}_{3} \mathrm{Sn}$ is still at an early stage of development. Tape-wound $\mathrm{Nb}_{3} \mathrm{Sn}$ could be used to provide a higher field in the plasma. Since there are no pulsed fields, eddy currents and the associated diamagnetic forces are not severe problems. However, provided it can generate the required field in the plasma region, the cheaper and more ductile NbTi is preferred in the present reactor study.

There are several advantages in going to a high current conductor. For example, this reduces the terminal voltage in the coil during discharge. It also reduces fabrication cost and improves winding accuracy. The operating current is $25 \mathrm{kA}$ for the present designs. This is a practical value based on technological expectations consistent with protection devices and conductor manufacturing techniques. For small monolithic conductors, the surface-to-volume ratio is large, and the conductor can be cooled sufficiently by having a reasonable fraction of its edge area exposed to liquid helium. But as the conductor is made larger, the surface-to-volume ratio is reduced, and edge cooling alone is not sufficient for cryostability. Furthermore, for a large conductor it is not economical to process the bulk of copper as matrix for the superconducting filaments, so that a large conductor is customarily built up from a multifilamentary composite with plain copper strips soldered onto the composite. Luton ${ }^{2}$ suggested that this copper strip should be formed with additional slots to increase the cooling surface area and cryostability of the conductor. The conductor design described below is a scaled-up version of such an extended-area conductor design.

\subsection{Description}

Figure E-7 shows an isometric view of a short length of the conductors described in Table E-2. The central composite is about twice the size of the largest composite currently available commercially, and could be made of two such composites located side by side. The superconductor current density needed $\left(18.82 \mathrm{kA} / \mathrm{cm}^{2}\right)$ is within the existing capability for operation at $4.2 \mathrm{~K}$ and $7.5 \mathrm{~T}$. Since the coils are not pulsed, superconducting filaments of relatively large size and twist pitch (e.g., 5 mils, 4 twists/ft) may be used. The copper/superconductor ratio in the composite is kept at the relatively low value of 2 to 1 to take advantage of the existence of the copper strip and to reduce the conductor cost. 


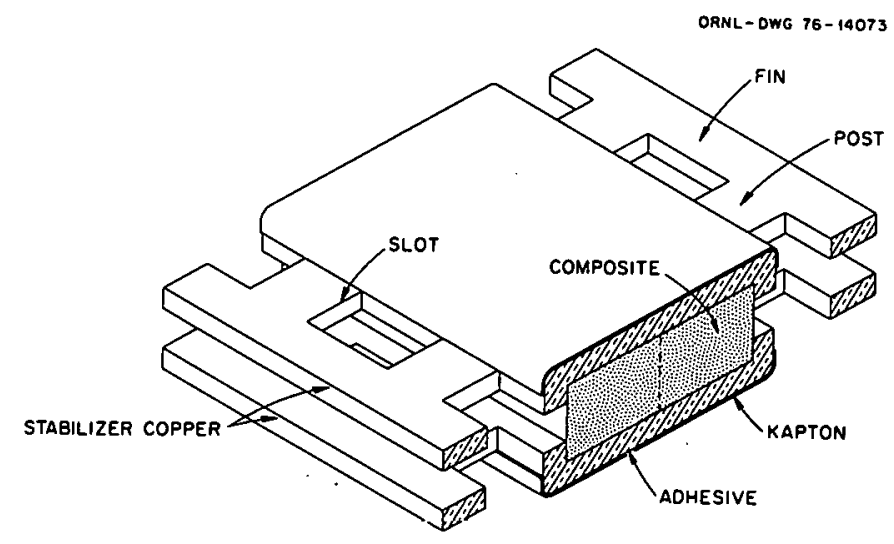

Fig. E-7. Extended-surface conductor design for EBTR toroidal magnets.

TABLE E-2

Conductor Design Parameters

\begin{tabular}{|c|c|c|}
\hline & EBTR-48 & EBTR-24 \\
\hline Overall width (in) & 1.48 & 1.42 \\
\hline Overall height (in) & 0.36 & 0.4 \\
\hline Composite width (in) & 0.66 & 0.66 \\
\hline Composite height (in) & 0.23 & 0.23 \\
\hline Average wetted perimeter $\left(i n^{2} / i n\right)$ & 2.57 & 2.48 \\
\hline $\mathrm{Cu} / \mathrm{SC}$ (in composite) & 2 & 2 \\
\hline $\mathrm{Cu} / \mathrm{SC}$ (overall) & 5.23 & 6.3 \\
\hline Design current (kA) & 6.25 & 6.25 \\
\hline $\begin{array}{l}\text { Superconductor current } \\
\text { density (at rated current) }\left(\mathrm{kA} / \mathrm{cm}^{2}\right)\end{array}$ & $18: 82$ & 18.82 \\
\hline Power per unit length (if a 11 & & \\
\hline current. flows in copper $)(W / \mathrm{cm})$ & 0.94 & 0.78 \\
\hline $\begin{array}{l}\text { Average surface power density (if all } \\
\text { current flows in copper) }\left(w / \mathrm{cm}^{2}\right)\end{array}$ & 0.14 & 0.12 \\
\hline $\begin{array}{l}{ }_{\mathrm{C}}^{\mathrm{Cu}}(4.2 \mathrm{~K}, 8 \mathrm{~T})(\Omega-\mathrm{cm}) \\
\text { Twist pit.ch of superconductor }\end{array}$ & $4.2 \times 10^{-8}$ & $4.2 \times 10^{-8}$ \\
\hline , filament (twist/ft) & 4 & 4 \\
\hline Filament diameter (mil) & 5 & 5 \\
\hline Number of filaments in conductor & 2622 & 2622 \\
\hline
\end{tabular}

One coolant passage is located between the fins at each edge of the conductor and provides azimuthal helium flow. The slots which connect with these passages allow radial flow. The most narrow flow restriction is $0.1 \mathrm{in}$. and is several times the breakaway bubble size $(0.02 \mathrm{in}.) .^{2}$ Table E-2 shows that under these assumptions, ${ }^{3}$ the peak nucleate pool-boiling heat transfer rate is $0.8 \mathrm{~W} / \mathrm{cm}^{2}$ and heat transfer occurs at the vertical surfaces, upward-facing horizontal surfaces, and downward-facing horizontal surfaces which are vapor covered $\left(0.13 \mathrm{~W} / \mathrm{cm}^{2}\right)$, while no heat conduction occurs down or 
between the conductors. The cooling surfaces are large enough so that the heat that can be transferred to the helium is four to six times the joule heating produced if all the current flows in the copper. Therefore, the conductor is cryostatically stable, and will recover if forced normal.

The conductor is insulated between turns by two 2-mil Kapton sheets. Because of the way the extended surfaces are formed, there is at least a 120-mil space between bare surfaces of copper, so that the turn-to-turn voltage standoff capability is high. Since this insulation is a thin, flat strip and because it is flat-wound, it should be possible to use material which is inorganic and therefore radiation-resistant.

The copper stabilizer strips must have slots punched and formed into channels and must be soldered onto the composite conductor. The forming can be done with rollers at the time of soldering. The peak value of radial compression in the structural portion of the conductor face is about $6.9 \mathrm{ksi}$ for both designs. The peak axial compressive stress in the copper strip due to the cumulative axial loads occurs at the post region of the conductor. Stress values are $11 \mathrm{ksi}$ and $11.5 \mathrm{ksi}$ for EBTR-48 and EBTR-24 respectively. Shorter slots $(0.2 \mathrm{in})$ reduce this stress to about 9 ksi. Further reduction can be achieved by using interleaved stainless steel strips.

\section{MAGNET DESIGN}

In contrast to the tokamak, the EBT has a Illajor radius which is large compared to the inner radius of the coils. Thus, the magnetic field and loading are nearly symmetrical. There is no need to use "D-shape". or oval-shape coils to minimize the bending moment, and coils with a circular shape are used. The transient heat loads that have been identified are minimal; therefore, the choice of pool-boiling cooling seems appropriate. The reason for the quiet thermal environment is that there are no pulsed fields and there is adequate space for shielding within moderate size coils.

The parameters of the EBT reactor toroidal magnets are given in Table E-3. As mentioned in Sect. 3 , the operating current will be $25 \mathrm{kA}$. To achieve this, four conductors will be interleaved along their wide faces and co-wound in parallel to make up a single turn. Variations in the current distribution in each conductor can be avoided by winding the four conductors ${ }^{4}$ symmetrically in a spiral as indicated in Fig. E-8.

The coils are fairly long compared with their radial thickness. Furthermore, their axis is horizontal. For layer-wound construction, in order to provide protection ayainst high layer-to-layer voltage differences, solid sheets of insulator are required between layers. This introduces long horizontal cooling channeis which tend to trap helium bubbles. Pancake-wound construction is preferred since it allows the helium bubbles to rise quickly to the top of the coil. A $0.1-1$. spdcer is pruvided between pancakes for insulation and for load transmission. Additional turn-to-turn voltage protection is provided by an insulating strip $0.13-0.14 \mathrm{~cm}$ long co-wound with each turn of the conductor. The winding is insulated from the coil case by insulation 0.56-0.75 cm thick.

Tensile (hoop) stress in the winding has been estimated by MARTON, ${ }^{5}$ a stress code for homogeneous isotropic solenoids. If the Young's modulus of copper $\left(17 \times 10^{6} \mathrm{psi}\right)$ is used for the winding region, then the peak stress and strain are $19.6 \mathrm{ksi}$ and $0.11 \%$ for EBTR-48 and $19.2 \mathrm{ksi}$ and $0.113 \%$ for EBTR-24. These values are within the allowable tensile stress for copper (25 ks $\mathrm{i}$ ) and the allowable strain for the $\mathrm{NbTi}$ superconductor (0.15\%). Additional structural support is not needed. The compressive axial stress is $4.31 \mathrm{ksi}$ for EBTR-48 and $3.75 \mathrm{ksi}$ for EBTR-24. These values pose no problem for spacers made from glass-reinforced composites. To improve the electrical insulation and to simplify winding construction, the interleaved structural strips are omitted. Instead of the strips, a 5-cm stainless steel wall is used as the coil case and at the same time provides additional structural support. The peak 
stress in the winding can be further reduced by applying tension during winding. Here, tight winding without potting is the preferred method for coil fabrication. The supports for gravity and centering forces are discussed in Appendix G.

TABLE E-3

\section{EBTR Toroidal Field Coil Design}

\begin{tabular}{|c|c|c|}
\hline & EBTR-48 & EBTR-24 \\
\hline Number of coils & 48 & 24 \\
\hline Major radius $(\mathrm{m})$ & 60 & 24 \\
\hline Peak field in winding $(T)$ & 7.28 & 7.43 \\
\hline Operating current (kA) & 25 & 25 \\
\hline \multicolumn{3}{|l|}{ Inside radius } \\
\hline coil case $(m)$ & 2.95 & 2.75 \\
\hline winding $(\mathrm{m})$ & 3.0 & 2.8 \\
\hline \multicolumn{3}{|l|}{ Outside radius } \\
\hline coil case $(m)$ & 3.65 & 3.45 \\
\hline winding (m) & 3.56 & 3.4 \\
\hline \multicolumn{3}{|l|}{ Half-axial length } \\
\hline coil casc & 1.3 & 1.1 \\
\hline winding $(\mathrm{m})$ & 1.25 & 1.05 \\
\hline Current density in winding $\left(\mathrm{kA} / \mathrm{cm}^{2}\right)$ & 1.55 & 1.5 \\
\hline Number of pancakes/coil & 62 & 54 \\
\hline Number of turns/pancakes & 14 & 14 \\
\hline Number of conductors/turn & 4 & 4 \\
\hline Length of conductor/coil $(A-m)$ & $4.47 \times 10^{8}$ & $3.68 \times 10^{8}$ \\
\hline Thickness of insulation stripe (between turns) $(\mathrm{cm})$ & 0.14 & 0.131 \\
\hline Thickness of spacer between pancakes $(\mathrm{cm})$ & 0.254 & 0.254 \\
\hline \multicolumn{2}{|l|}{ Insulation between winding and coil case $(\mathrm{cm})$} & \\
\hline face parallel to coil axis & 0.635 & 0.635 \\
\hline face perpendicular to coil axis & 0.56 & 0.75 \\
\hline
\end{tabular}

An Interesting possibility is that the magnets may be made modular and used in reactors of different sizes. As shown in Table E-4, as Iong as the ratio of major radius to the number of colls is fixed, the field strengths produced by modular magnets of the same size are not sensitive to the stze of the reactur. This ratio also fixes the length of the blanket and shield modules which are lociated between adjacent magnets. Furthermore, for a given neutron wall loading (MW/m²), the plasma radius is relatively insensitive to changes in the major radius of the machine. There is a strong indication that various components of the reactor system can be modular and can be standardized and still serve in reactors of different majur radij and power outputs.

As mentioned in Appendix G, the modular design of the blanket and shield allows first wall maintenance operations that do not disturb the coil. Because of the complexity and time required to make sound electrical connections ( $10^{-8} \mathrm{ohm}$ ) with remote handling equipment, efforts to minimize these operations are desirable. 


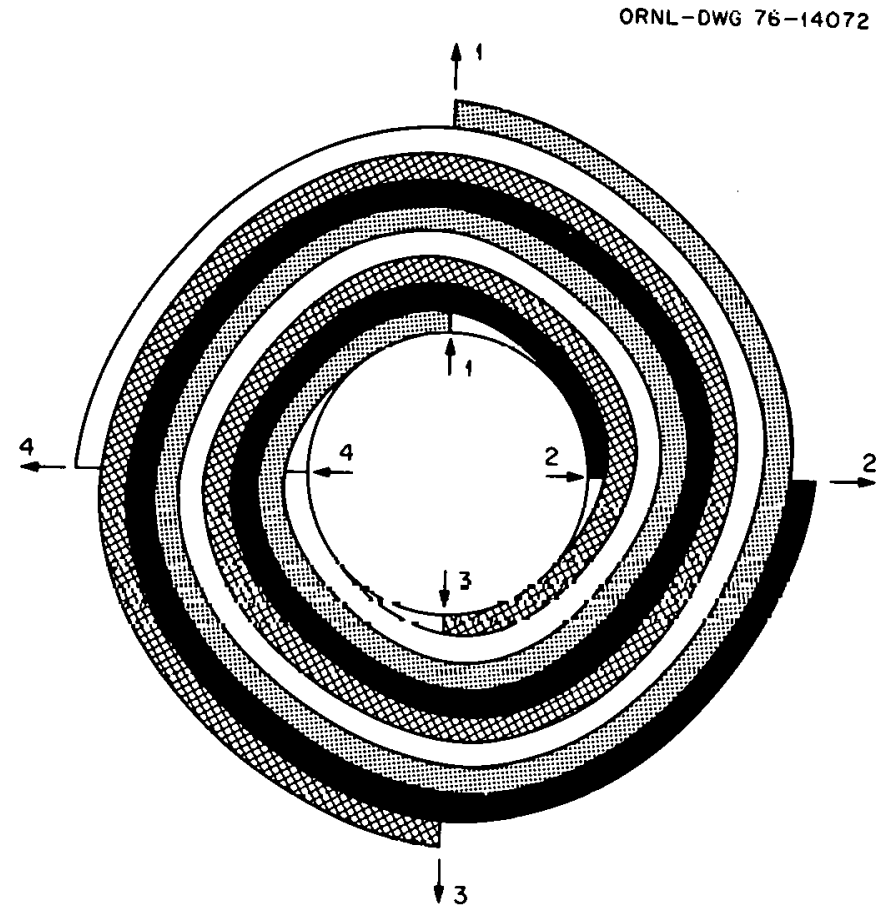

Fig. E-8. Schematic diagram for spiral winding of four conductors in parallel in a pancake.

TABLE E-4

Magnetic Field Values for Same Winding Parameters But Different Major Radius

\begin{tabular}{|c|c|c|c|c|c|}
\hline & $\begin{array}{l}\text { Number } \\
\text { of } \\
\text { Culls } \\
\end{array}$ & $\begin{array}{l}\text { Maior } \\
\text { Radius } \\
\text { (m) } \\
\end{array}$ & $\begin{array}{l}\text { Mirror } \\
\text { Katíno } \\
\end{array}$ & $\begin{array}{l}\text { Fipld at } \\
\text { Midplane } \\
\text { (T) } \\
\end{array}$ & $\begin{array}{c}\text { Max,imum Field } \\
\text { iii Windiny } \\
\text { (T) }\end{array}$ \\
\hline & 48 & 60 & 1.77 & 2.5 & 7.28 \\
\hline \multirow{6}{*}{$\begin{array}{l}\text { Major radius/number of coils = } \\
1.25 \mathrm{~m} \text {, winding parameters } \\
\text { same as EBTR- } 48 \text { design. }\end{array}$} & 24 & 30 & 1.77 & 2.5 & 7.32 \\
\hline & 72 & 90 & 1.77 & 2.5 & 7.27 \\
\hline & 96 & 120 & 1.77 & 2.5 & 7.26 \\
\hline & 36 & 15 & 1.77 & 2.5 & 7.30 \\
\hline & 50 & 62.5 & 1.77 & 2.5 & 7.28 \\
\hline & 24 & 24 & 1.45 & 3.10 & 7.43 \\
\hline \multirow{5}{*}{$\begin{array}{l}\text { Major radius/number of coils - } \\
1 \mathrm{~m} \text {, winding parameters samc } \\
\text { as EBTR-24 design. }\end{array}$} & 30 & 30 & 1.45 & 3.10 & 7.40 \\
\hline & $4 n$ & 48 & 1.15 & 3.10 & 7.36 \\
\hline & 60 & 60 & 1.45 & 3.10 & 7.35 \\
\hline & 70 & 70 & 1.45 & 3.10 & 7.34 \\
\hline & 96 & 96 & 1.45 & 3.10 & 7.33 \\
\hline
\end{tabular}

\section{CRYOGENICS}

The toroidal magnets in the EBT reactor will be cooled by liquid helium under pool-boiling conditions. Circulating pumps will be used to assure some flow of helium to the magnets for the 
natural circulation system. To avoid interference with the remote assembly of the blanket, helium will be supplied to the magnets in the radial direction from the cold box. Each cold box (rated $3 \mathrm{~kW}$ at 4.2 K) will supply the helium required by eight magnets. Each coil will have its own dewar and vacuum vessel, and there is no connection for helium flow between coils. cold boxes will, however, be interconnected to allow for back-up operation in case the helium supply in one of the cold boxes runs low. To avoid excessive losses in the vapor-cooled current leads, the current leads between coils which use the same power supply will be run in conduits cooled to $4.2 \mathrm{~K}$. Liquid helium will be fed to the bottom of each magnet and will flow out of the top. This will facilitate the removal of any helium bubbles formed.

The EBT reactor is expected to operate at steady state. Accordingly, there will be no heat load requirement due to pulsed fields. The heat loads that must be removed by the liquid helium cryogenic system include the radiation heat absorbed from the magnet dewar surface, the loads due to conduction through the support and instrument leads, and the radiation energy absorbed from incident neutrons and gamma rays.

The coils have a large surface area, since they are about $2.5 \mathrm{~m}$ in length and about $3 \mathrm{~m}$ in bore radius (EBTR-48). Radiation heat losses are kept to a value of about $10 \mathrm{~W} / \mathrm{magnet}$ by using vacuumjacketed and $\mathrm{LN}_{2}$-cooled dewars and superinsulation around the magnets. (An average radiation loss of $7.5 \mu \mathrm{W} / \mathrm{cm}^{2}$ is assumed over the magnet surface.)

The total neutron and gamma heating in the magnets, assuming a neutron wall loading of $1 \mathrm{MW} / \mathrm{m}^{2}$, was estimated to be $96.4 \mathrm{~W}$ and $76 \mathrm{~W}$ per coil for EBTR-48 and EBTR-24 respectively. A $70-\mathrm{cm}-$ thick shield was used to provide an energy attenuation factor of $2 \times 10^{-6}$ (see Appendix $H$ ). The average power density in the windings due to this energy deposition is about $1.28 \times 10^{-6} \mathrm{~W} / \mathrm{cm}^{3}$ and should pose no heat transfer or vapor removal problems.

The heat input to the vaporized helium used for vapor-cooling the leads is about $2.8 \mathrm{~W} / \mathrm{kA}$ per pair, ${ }^{2}$ with a refrigeration load about five times this value. (The helium boil-off rate may be estimated at $3 \mathrm{l} / \mathrm{hr}$ per pair of 1-kA leads.) Thus a pair of 25-kA leads will need $0.35 \mathrm{~kW}$ of refrigeration. If the pair of leads is only used for discharging the coil and not to carry current during normal operation, its refrigeration load is estimated to be $0.12 \mathrm{~kW}$ per pair.

The load due to heat conduction through the structural supports is conservatively estimated to be $35 \mathrm{~W} / \mathrm{coil}$. This loss can be reduced by providing liquid nitrogen $\left(\mathrm{LN}_{2}\right)$ cooling for the supports. Each cold box will need a $125-W$ circulating pump to pump the helium through the coils. The estimated heat loads on the liquid helium cooling systemis are summarized in Table E-5.

TABLE E-5

Magnet Cooling Requirements for EBTR

$\begin{array}{lcc} & \text { EBTR-48 } & \text { EBTR-24 } \\ \text { Conduction to structure (kW) } & 1.68 & 0.84 \\ \text { Thermal radiation (kW) } & 0.4 \dot{9} & U .20 \\ \text { Pumping circulation (kW) } & 0.75 & 0.38 \\ \text { Neutron and gamma heating (kW) } & 4.63 & 1.82 \\ \text { Vapor-cooled leads, (kW) } & & 1.05 \\ \text { Active leads } & 2.1 & 2.52 \\ \text { Passive leads (for use in discharge) } & 10.8 & 6.81 \\ \text { Total loads (at } 4.2 \mathrm{~K})(\mathrm{kW}) & 20.45 & \end{array}$


Note that, because of the segmentation requirement for EBTR-48, which introduces additional passive current leads, its cryogenic load is more than doubled compared to the EBTR-24 load. For this reason, one spare cold box will be needed for EBTR-48. The passive lead loss can be cut down considerably by providing intermediate cooling at $\mathrm{LN}_{2}$ temperatures.

\section{PROTECTION DESIGN}

\subsection{Design Criteria}

As mentioned in previous sections, in order to reduce the terminal voltage at the magnet during discharge, a high operating current $(25 \mathrm{kA})$ is used. Modular power supplies of $25 \mathrm{kA}, 20 \mathrm{~V}$ are used to charge eight magnets in series. Six units are needed for EBTR-48 and three units are needed for EBTR-24. Charging times are about $16 \mathrm{hr}$ and $13 \mathrm{hr}$ respectively. As discussed elsewhere, ${ }^{6}$ the temperature rise, the terminal voltage, and the mechanical loading need to be within sate limits during a coll quench.

Dump resistors will be used to discharge the energy stored in the coil during a quench. Each coil needs to be discharged through its own dump resistor. 0therwise, the terminal voltage for eight coils discharged in series would be too high. If one coil has quenched, discharging all 48 coils in the system (EBTR-48) is proposed, instead of keeping them in operation. There are several reasons for this. First, there is no incentive to keep the rest of the coils in operation, since all coils are necessary for plasma operation, and the time to recharge the coils is not excessively long. Second, if coils in $i$, toroidal set are kept in operation while one of them is quenched, large out-of-plane loadings and lateral displacements will be induced in the coils adjacent to the one that has quenched. Third, the current will change in the unquenched coils due to inductive coupling. This will require a further readjustment before the whole system can be brought back to normal operation again.

\subsection{Description}

Voltage taps are used on each coil as the main quench detection device. Without external pulsed fields, the only inductive voltage that should be monitored exists during the charging phase of operation. Pick-up coils at the current leads of the power supplies can be used to compensate for the inductive voltage. Sensitivities of detecting resistive signals which are less than $0.1 \%$ of the inductive voltage have been demonstrated. ${ }^{7}$ Other transducers such as strain gauges and temperaturepressure sensors can also be used to improve reliability. As soon as the quench signal exceeds the set voltage and time duration, the discharge sequence will be triggered automatically.

The discharge sequence can best be illustrated by Fig. E-9. Coils (inside dotted line) are at liquid helium temperature, while everything else is at room temperature. In normal operation, all $S_{1}$ switches are open and all $\mathrm{S}_{2}$ switches are closed. A diode is placed in front of each coil's dump resistor $\left(R_{1}\right)$ to allow proper charging during normal operation. To discharge, all $S_{1}$ switches are closed first to provide a current bypass and to protect the power supplies; then all $\mathrm{S}_{2}$ switches are opened to isolate the coils and to force the current in each coil to flow through its dump resistor. The maximum voltage in the system is the terminal voltage across one coil. After discharging is complete, $S_{1}$ will be opened and $S_{2}$ closed, and recharging can then resume.

The use of the dump resistors allows the extraction of the most energy from the coil, and thus keeps the time required for cooldown after quenching to a minimum. In Table E-6, an upper bound for the maximum temperature rise inside the coil during discharge is given. The enthalpy of liquid helium is ignored in this estimate. The actual temperature rise is likely to be much less than $100 \mathrm{~K}$, 
especially for coils constructed with good coolant flow and without potting. The limit is chosen as $\Delta T=100 \mathrm{~K}$, so that the corresponding maximum possible thermal strain induced by quenching $\left(5 \times 10^{-4}\right)$ is well within the tolerable limit. The duration of discharge is in the range of minutes and is adequate to allow the plasma to be purged. The response time of instrumentation must be in the millisecond range. For EBTR-48, the voltage across each coil during the discharge is quite high. Thus it is desirable to reconnect each coil into two independent sections during discharge. Each section will have a dump resistor with one-half $(0.043 \Omega)$ the total dump resistance. In this way, the maximum voltage in EBTR-48 can be kept to the level of $1 \mathrm{kV}$. Recently, such a scheme has been verified experimentally. ${ }^{8}$ For EBTR-48, instead of the eight sections shown in Fig. E-9, sixteen sections will be used, each discharging one-half of a coil and in parallel with other sections. The $\mathrm{S}_{2}$ switches located between two sections may be replaced by superconducting switches for persistent operation. This would reduce lead loss, assumed in Sect. 5. If the mirror ratio and field pattern turn out to be critical, one may want to use one power supply for each coil in order to be able to adjust the current level in each coil independently. In that case, the protection circuitry is further simplified, since each coil will be discharged independently. However, lead loss will increase. The mechanical load during the fault is covered in Appendix $G$ and will not be discussed further here.

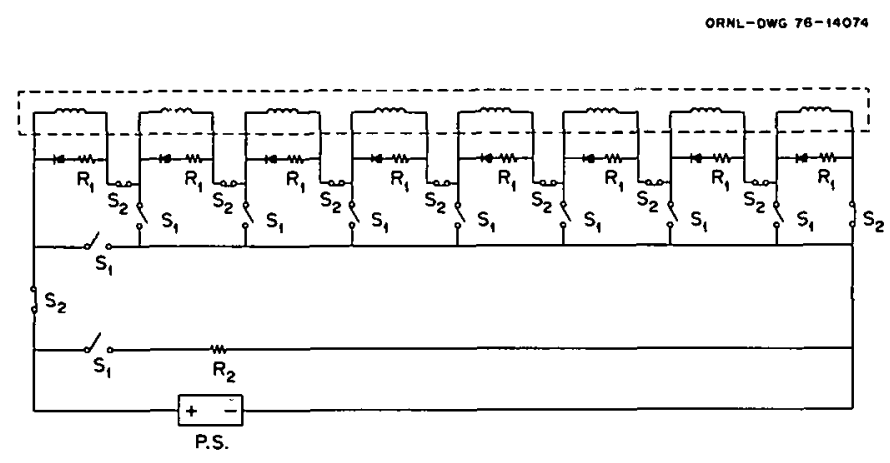

Fig. E-9. EBTR protection scheme, one section (eight magnets).

TABLE E- 6

Protection Characteristics of EBT Reactor Coils

\begin{tabular}{|c|c|c|}
\hline & EBTR-48 & EBTR-24 \\
\hline \multicolumn{3}{|l|}{ Coil Parameters } \\
\hline Total energy stored/coil (GJ) & 1.84 & 1.43 \\
\hline Bath temperature $(\mathrm{K})$ & 4.2 & 4.2 \\
\hline Dump resistance/coil $(\Omega)$ & 0.086 & 0.045 \\
\hline \multicolumn{3}{|l|}{ Calculated Protection Characteristics } \\
\hline Voltage difference in coil (V) & 2153 & 1127 \\
\hline Turn-to-turn voltage (V) & 2.5 & 1.5 \\
\hline Coil dumping duration $(s)$ & 68.4 & 101.6 \\
\hline Upper bound for the maximum temperature in coil (K) & 100 & 100 \\
\hline Upper bound for the maximum thermal strain in coil & $5 \times 10^{-4}$ & $5 \times 10^{-4}$ \\
\hline Number of sections needed per coil & 2 & 1 \\
\hline
\end{tabular}


7. EBTR-48 ALTERNATE MAGNET DESIGN

\subsection{Conductor Design}

The choice of an average current density (symbolized by $\langle J\rangle$ ) in the magnet coils is influenced by the following factors:

A. Cost and weight of the magnet,

B. Magnet stability:

1. maximum field at the windings,

2. type of superconducting (SC) wire used ( $\mathrm{NbTi}$ or $\mathrm{Nb}_{3} \mathrm{Sn}$ ),

3. type of cooling system, i.e., pool-boiling (liquid $\mathrm{He}^{4}$ ) or forced-flow (supercritical $\mathrm{He}^{4}$ ),

C. Refrigeration and pumping power requirements.

In examining the trade-offs involved in choosing a current density, two different magnet designs

were considered. The reactor reference design assumes an averaqe current densitv of $1550 \mathrm{~A} / \mathrm{cm}^{2}$. and the alternate design uses a suitably distributed higher current density. Details of the alternate design are given in Table E-7.

TABLE E-7

Parameters for Alternative (EBTR-48) Magnet Design

$<\mathrm{J}>\left(\mathrm{A} / \mathrm{cm}^{2}\right)$

Inner windings 2000

Outer windings $\quad 4000$

Magnetic field at magnet throat

(from 1 magnet) $(T)$

From inner windings $\quad 1.5$

From outer windings 2.5

Length ( $m$ )

Bore radius (m)

Wire type

Cooling system

1.5

3

$\mathrm{Nb}_{3} \mathrm{Sn}$ (bundle conductor)

Forced flow, supercritical He

Maximum magnetic field

At inner winding ( $T$ )

10.1

At outer winding ( $T$ )

5.2

Nunber of coils in reactor

48

Mirror ratio

Distance between magnets $(\mathrm{m})$

7.6

In contrast to the reference design, the higher current density in this design places more severe requirements on ensuring the stable operation of the coil. The higher maximum magnetic field increases the magnetoresistance of the copper and the higher $\langle\mathrm{J}\rangle$ requires an increased heat transfer rate between the copper and $\mathrm{He}^{4}$ during a quench. The requirement is greater than that obtained with pool-boiling cooling. The requisite increase in the heat transfer rate is accomplished by using supercritical He 4 and forced flow through a bundle conductor. (In the bundle conductor concept, a number of copper strands containing multifilaments of $\mathrm{Nb}_{3} \mathrm{Sn}$ are twisted into a cable and surrounded by a vacuum conduit. (For further details see Ref. 9.) In this forced-flow design He mass flow rates and the associated pressure drop will be increased relative to the pool-boiling design. 
An advantage of $\mathrm{Nb}_{3} \mathrm{Sn}$ wire compared to $\mathrm{NbTi}$ wire is that the current-sharing temperature zone is higher by several degrees Kelvin. The $\mathrm{Nb}_{3} \mathrm{Sn}$ wire can also operate in fields up to about $15 \mathrm{~T}$ compared to a maximum field of about $9 \mathrm{~T}$ for $\mathrm{NbTi}$ wire. The alternate design, with a maximum field at the inner windings of $10.1 \mathrm{~T}$, necessitates the use of $\mathrm{Nb}_{3} \mathrm{Sn}$ wire. The main disadvantage of $\mathrm{Nb}_{3} \mathrm{Sn}$ wire is that it is currently more expensive than $\mathrm{NbTi}$ wire. Multifilament $\mathrm{Nb}_{3} \mathrm{Sn}$ is a new technology and the wire still has large developmental costs associated with it. Under the assumption that bulk tin costs no more than titanium, it is possible that once these development costs diminish, the price of $\mathrm{Nb}_{3} \mathrm{Sn}$ wire will be compatible with that of NbTi wire.

Once the choice of $\mathrm{Nb}_{3} \mathrm{Sn}$ wire is made, the forced-flow cooling method (using supercritical He ${ }^{4}$ ) offers a very attractive advantage. The coolant temperature can be increased to $5 \mathrm{~K}$, still below the current-sharing region. If NbTi were used, a coolant temperature of about $2.5 \mathrm{~K}$ would be needed to ensure the same degree of stability. Thus a factor of two can be saved in the refrigeration power requirements, assuming a fixed percentage in the efficiency of a Carnot-cycle refrigerator. Earlier. it was mentioned that the forced-flow cooling method would have higher pumping power requirements compared to pool boiling due to the higher pressure drop. The pumping power still is estimated to be only about $5-10 \%$ of the refrigeration power requirements. Thus, forced-flow cooling should require less power to run than a pool-boiling system.

A final advantage of the higher current density design is that less superconducting wire will be needed. This will make the magnet assembly lighter and less expensive. This, of course, assumes that the development of bundle conductors and $\mathrm{Nb}_{3} \mathrm{Sn}$ has been completed. The magnet modules might also be smaller, which will ease construction and maintenance procedures. Thus it appears that the use of forced-flow cooling will offer many advantages if research and development proves its engineering feasibility.

\subsection{Force Calculations}

The forces in the EBTR-48 magnet configuration, using the alternate magnet design, were calculated. The configuration used in these calculations included four divertor assemblies which were located symmetrically around the torus. The divertor assembly includes one divertor coil and two shaping coils (see Appendix $F$ for details). The specifications for the coils are shown in Table E-8.

TABLE E-8

Specifications for Divertor and Shaping Coils

\begin{tabular}{lcc} 
& $\begin{array}{c}\text { Divertor } \\
\text { Coil }\end{array}$ & $\begin{array}{c}\text { Shaping } \\
\text { Conil }\end{array}$ \\
\cline { 2 - 3 } & & 0.5 \\
Length $(\mathrm{m})$ & 1 & 2.3 \\
Radius $(\mathrm{m})$ & 3 & 1 \\
Field (on axis) (T) & 2 & +2000 \\
Average current density $\left(\mathrm{A} / \mathrm{cm}^{2}\right)$ & -2000 &
\end{tabular}

(Note that positive currents are in the same direction as those in the main EBT coils.)

Radial and azimuthal forces on the magnet coils were computed by dividing the current in each coil into a number of current-carrying loops. The results of these calculations are shown in Table E-9. Error estimates were made by repeating the calculations with a tenfold increase in the fineness of the wire mesh carrying the current. 
If an individual coil fails, the azimuthal force on the adjacent coils will be on the order of $60 \mathrm{MN}$ and the radial force will be less than in the case prior to failure. Ideally, if a forced shutdown mechanism deenergizes all the magnets simultaneously when a quench occurs, large failure-mode forces will not exist. The forced shutdown mechanism is discussed in Sect. 7.3.

\subsection{Forced Shutdown}

When one of the main EBT coils in the alternative design has quenched, it is desirable to deenergize both the quenched coil and the other reactor coils simultaneously and as quickly as possible. This will prevent the occurrence of unbalanced forces between the coils. It also will prevent a large heat deposition in the quenched coil. Each coil has $2200 \mathrm{MJ}$ stored in its magnetic field and a total of 36 tons of copper and 57 tons of stainless steel reinforcement ribbon in its structure. If all of the magnetic energy were distributed uniformly as heat into this material, the temperature would increase from $\sim 4$ $K$ to $\sim 148 \mathrm{~K}$. It is desirable from a refrigeration viewpoint to dump this energy into an external sink, and thus save the energy that otherwise would be needed to remove the heat from the magnet itself.

The limiting factor on the speed at which the coils can be deenergized is the voltage drop between the magnet pancakes and the dewar. In designing the winding structure, this voltage drop was kept below $4000 \mathrm{~V}$.

The shutdown equations for a model that includes the temperature variation of all the physical properties in both magnet and dump resistor were solved and it was found that the worst case of fully quenched emergency shutdown can be accommodated by use of the 15-tonne aipha iron resistor. The resistor was assumed to be noninductively wound, to have a surface area of $10^{2} \mathrm{~m}^{2}$, and to be cooled only by radiation during the shutdown transient. The voltage transient is illustrated in Fig. E-10. The maximum voltage drop incurred in this case was $3822 \mathrm{~V}$ and of the initial $2200 \mathrm{MJ}$ in the coil, $1986 \mathrm{MJ}$ were deposited in the external resistor (i.e., only $7 \%$ remained in the coil and its structure).

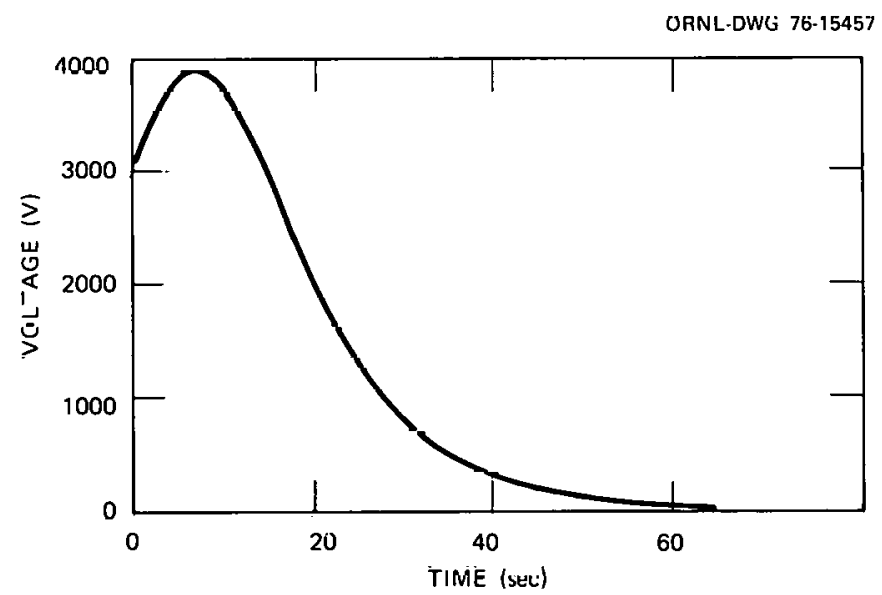

Fig. E-10. Coil terminal voltage vs time for forced shutdown of alternate 48-coil design.

\subsection{Dewars for Alternate Magnet Design}

The dewars housing the superconducting coils in the EBT reactor must fulfill three important functions. First, the coil, operating at $4 \mathrm{~K}$, must be thermally insulated from its immediate surroundings, which are operating at $350 \mathrm{~K}$. Second, the dewar must provide mechanical support for the coil both 
during reactor assembly and operation. In one reactor design concept where the coils and blanket are removed as integral modules for first wall and blanket repair, the dewar must provide support and physical protection for the coil during remote maintenance. Third, the dewar must provide lateral restraint and thermal protection in case one or more of the adjacent coils quenches or loses coolant flow. In this accident situation the coil is subjected to a lateral force of about 60 MN which must be borne in turn by the dewar.

The proposed dewar design is shown in Figs. G-10 and G-11 in Appendix G. The dewar consists of two austenitic steel shells. The outer shell operates at about $350 \mathrm{~K}$ and the inner cold shell at $4 \mathrm{~K}$. The warm and cold shells are separated by an evacuated annulus in which aluminized mylar and a copper shield at $80 \mathrm{~K}$ serve as insulation.

The coil is supported by sixteen $2.25-\mathrm{m}-1$ ong, $7.61-\mathrm{cm}$-diameter tendons. The tendons are constructed of $304 \mathrm{~L}$ stainless steel and are stressed to one half of their yield strength when the reactor is in operation. At a point about $1 \mathrm{~m}$ from the warm end of the tendon, a liquid-nitrogen-cooled collar is placed around the tendon to minimize the power consumed by the refrigerators. The electrical refrigeration work required, $W_{E}$, is given by,

$$
W_{[}=800 q_{\text {lle }}+50 q_{N_{2}}
$$

where $q_{N_{2}}$ and $q_{H e}$ are the heat leaks into the nitrogen and helium respectively. This equation assumes that the refrigerators operate at $\sim 10 \%$ of Carnot efficiency. The heat leak is further reduced by using a nylon bushing [stressed to $20 \mathrm{ksi}$ (138 MPa) in compression] at the point where the tendon attaches to the cold shell of the dewar.

In addition, shorter tendons are provided to restrain the coil against the lateral forces that are caused by the asymmetries in the magnetic field due to curvature. These forces have been calculated for a 44-coil, 4-divertor system and are shown for one octant in Table E-9.

TABLE E-9

Forces on the Reactor Coils (Normal Operating Conditions) ${ }^{\mathrm{a}}$

\begin{tabular}{lcc} 
coil & $\begin{array}{c}\text { Azimuthal Force } \\
(\mathrm{MN},+1.5 \%)\end{array}$ & $\begin{array}{c}\text { Radial Inward Force } \\
(\mathrm{MN},+0.5 \%)\end{array}$ \\
\cline { 2 - 2 } & 0.0 & -3.81 \\
Shaping coil & 51.41 & -0.17 \\
Main coil \#1 & 54.38 & 4.42 \\
Main coil \#2 & 5.92 & 7.53 \\
Main coil \#3 & 1.32 & 8.06 \\
Main coil \#4 & 0.41 & 8.20 \\
Main coil \#5 & 0.14 & 8.25 \\
Main coil \#6 & 0.0 & 8.26
\end{tabular}

${ }^{a} 44$ main coils and 4 divertor assemblies have been included in the calculations.

The lateral support tendons have been designed to withstand the lateral forces experienced in positions 2 through 5 as defined in Table E-9. The coil used in position 1 must be connected to the warm shell by means of a cold tendon system as shown in Fig. G-11. The presence of this auxiliary' support system and the fact that the cryopumping systems interfere with the removal of coil-blanket modules directly adjacent to the divertors may mean that the divertor plus two adjacent modules must be 
built as one unit. In this case, the first wall and blanket could be removed for repair without moving the coil, which is the procedure used for the fixed coil design (see Appendix G).

The insulation chosen for the dewar is an aluminized mylar film wrapped in layers around the coil in the space between the warm shell and the cold shell. A thin copper shield, cooled by liquid nitrogen flowing through attached tubes, reduces the heat leak into the liquid nitrogen and thereby minimizes the power needed for refrigeration. After assembly of the coil and dewar, the volume between the shells is evacuated.

At the ends of the dewar, the coil will contact impact pads under certain very extreme accident conditions. These pads are separated by a small gap during normal operation of the reactor, and then the only heat leak is due to radiation. To reduce these losses, a copper shield at $80 \mathrm{~K}$ has been positioned in the gap.

The heat leak into the dewar due to the coil leads has been estimated using the formula given by Montgomery 10 for optimized leads, and the energy deposition in the winding from the neutrons and gamma rays coming through a $70-\mathrm{cm}$ shield has been included (see Appendix $\mathrm{H}$ ).

A summary of the expected heat leaks and refrigerator power requirements is given in Table E-10.

TABLE E-10

Heat Leaks into One EBT Dewar

(Calculated for one coil)

\begin{tabular}{|c|c|c|c|}
\hline Source: & $\begin{array}{c}{ }_{\mathrm{N}_{2}} \\
\text { Heat Leak } \\
\text { into [iquid } \mathrm{N}_{2} \\
(\mathrm{~W}) \\
\end{array}$ & $\begin{array}{c}{ }^{\mathrm{q}_{\mathrm{He}}} \\
\text { Heat Leak } \\
\text { into Liquid He } \\
(\mathrm{W}) \\
\end{array}$ & $\begin{array}{l}\quad W_{E} \\
\text { Refrigeration } \\
\text { Power Required } \\
(\mathrm{kW}) \\
\end{array}$ \\
\hline $\begin{array}{l}\text { Matn suppurt } \\
\text { tendons }\end{array}$ & 228 & $22 . \dot{9}$ & 29.7 \\
\hline $\begin{array}{l}\text { Latcral } \\
\text { support } \\
\text { tendon }\end{array}$ & 62.24 & 4.96 & 1.08 \\
\hline Insulation & 27.4 & 3.62 & 4.26 \\
\hline $\begin{array}{l}\text { Impact pads } \\
(\text { no contact) }\end{array}$ & 44.4 & 0.145 & 2.34 \\
\hline Leads & -- & $90 \mathrm{~W}$ & 72 \\
\hline $\begin{array}{l}\text { Neutrons } \\
\text { gamma energy } \\
\text { deposition }\end{array}$ & -- & 24.9 & 19.9 \\
\hline TOTAL & 362.0 & 146.5 & 135.3 \\
\hline
\end{tabular}

\section{SUMMARY OF MAGNET DESIGN}

In summary, the toroidal magnet system for the EBT reactor seems feasible in view of existing technological capabilities. The large, high current conductor requires a moderate development effort which is currently under way in the Superconducting Magnet Development Program at ORNL. The forced-flow bundle conductor is currently being developed by the MIT Francis Bitter National Magnet Laboratory under subcontract with ORNL. The magnet requirements for the divertor will depend on the final design and location. The divertor magnet design will be investigated in more detail in the next fiscal year. 
1. C. S. Gardner, Toroidal Vacuum Field with Minimum Variation of $6 d \ell / B$, MATT-391, Princeton, New Jersey (December 1965).

2. J. N. Luton (ORNL), private communication, 1976.

3. C. G. Lawson' (ORNL), private communication, 1976.

4. J. N. Luton, "Some Engineering Problems in the Production of Stationary Magnetic Fields," Ph.D: Thesis, University of Tennessee.

5. W. H. Gray (ORNL), private communication, 1976.

6. H. T. Yeh, Consideration of Coil Protection and Electrical Connecting Schemes in Large Superconducting Toroidal Magnet System, ORNL/TM-5043, Oak Ridge (1976).

7. S. S. Shen and H. T. Yeh, "Analysis of Pulse Loss Measurement on NbTi Solenoid," paper presented at the 1976 Applied Superconductivity Conference, Stanford, California, April 1976.

8. H, T. Yeh, J. N. Luton, and J. E. Simpkins, "Current Averaging and Coil Segmentation in the Protection of Large Toroidal Superconducting Magnet Systems," paper presented at the 1975 Cryogenic Engineering Conference, Kingston, Canada, 1975.

9. M. 0. Hoenig and D. B. Montgomery, Forced Supercritical Helium Cooling for Large Toroidal Coils, Phase I Report, Francis Bitter National Magnet Laboratory, Massachusetts Institute of Technology, Cambridge, Massachusetts (April 1975).

10. D. B. Montgomery, Solenoid Magnet Design, Wiley-Interscience, New York, 1969. 


\section{INTRODUCTION}

Divertors may be required in a fusion reactor to provide for the removal of plasma reaction products, to act as conduits for steady state removal of diffusing plasma as well as access for initial pump down, and to prevent atoms sputtered from the system boundaries from diffusing deeply into the plasma. The divertors will intercept most of the charged particle and sustenance energy delivered to the plasma that does not escape as radiation or charge exchange neutrals. An efficient divertor can thus reduce the total first wall heat flux by factors of three or more. (For reasonably thin walls, the thermal flux is several times larger than the neutron heat load deposited in the first wall.)

The high aspect ratio and relatively low magnetic field of EBT make it possible to design a relatively simple "classical toroidal divertor." In the following sections the design criteria that a successful divertor must meet are discussed. Also presented is a detailed divertor design compatible with the standard module and magnet design of EBTR-24 (with $R_{0}=30 \mathrm{~m}$ ) and EBTR-48 reference designs.

\section{DIVERTOR REQUIREMENTS}

The magnetic field geometry of the divertor must be such that the separatrix can be accurately placed at the plasma boundary with relatively small field perturbations inside the limiting radius and field curvatures which are as gentle as possible outside the separatrix. The diverted field lines in the scrape-off layer should be manipulated in the body of the divertor so that the escaping plasma energy is uniformly distributed over the surface that intercepts it. The region through which the divertor field lines penetrate the first wall should be as small as possible to minimize any chance of gas back-streaming into the plasma and to avoid excessive neutron streaming into and through the divertor.

In addition to these requirements on magnetic field geometry, the divertor must have sufficient heat transfer area to handle the full steady state load with provision for handling brief overloads in the event of an emergency plasma shut down. The divertor also must have sufficient gas pumping surface (shielded from the thermal load) to pump away the neutralized gas after the escaping plasma strikes the thermal transfer surface. Because of the reversed field coils, the forces on the divertor will be large. The design, therefore, must be consistent with the requirements of mechanical support and access. The main field reversal coils will almost.certainly have to be superconducting, and it. is desirable for the shaping coils also to be superconducting, if possible.

In addition to the requirements listed above, additional conditions for the EBT reference design are imposed. It is required that the divertor design be such that it is compatible with the standard EBT module, be capable of staged multiple use so that three of the four divertors in the reference design would be capable of handling the total load, and that it use the same coolant for the thermal recovery panels in order to simplify the heat exchange system.

\section{DIVERTOR FIELD DESIGN}

The design process is illustrated here with a divertor compatible with the movable coil, lithium cooled, concrete shielded version of EBTR-48. The divertor would be changed little if it was designed to be compatiblc with the fixed coil nitrate cooled EBTR-48. The mechantcal design of divertors for 
the more highly curved systems is similar, but greater care must be taken to ensure that the effective system aperture is maintained.

In the EBT divertors described here, field reversal is accomplished by reversing the current in one of the standard coils. In general, the reversed current required in this coil is less than the standard current by a factor $1.8-2$. For the same overall current density this coil can be smaller in cross section than one of the standard coils.

In order that the field intensity be maintained at the plasma center line under the reversal coil, two additional "shaping" coils are required. These are positioned on either side of the reversal coil and approximately one-quarter to one-half of a reactor section length away from the reversal coil $(\theta \approx \alpha / 4-\alpha / 2)$. The current in each of these coils is about one-half of the current in the reversal coil.

The three coils (two shaping and one reversal) are now adjusted so that the required field lines are diverted. For a plasma radius of $1.0 \mathrm{~m}$ and a given wall radius of $1.2 \mathrm{~m}$, the objective is to create a field null under the reversal coil at a radius of $1.0 \mathrm{~m}$ from the plasma center line. Once the field lines have been diverted, they must be separated by reducing the field sufficiently. This is achieved by using a set of low current "tickling coils" with radi $i$ about $2-2.5$ times that of the standard coil radius.

Figure F-1 shows two field lines which would normally be at $1.0 \mathrm{~m}$ and $1.2 \mathrm{~m}$ from the reactor center line between mirror sections. The quantity $I_{c}$ refers to the total current in one of the standard coils. The results are dependent on the ratios of the currents used in this study and can be scaled from $I_{C}=6000 \mathrm{~A}$. The design presented here was calculated in linear geometry for point coils. For a $60 \mathrm{~m}$ major radius reactor, errors introduced are small and can be corrected simply. Basically, the design presented here provides a take-off point for a more detalled future design.

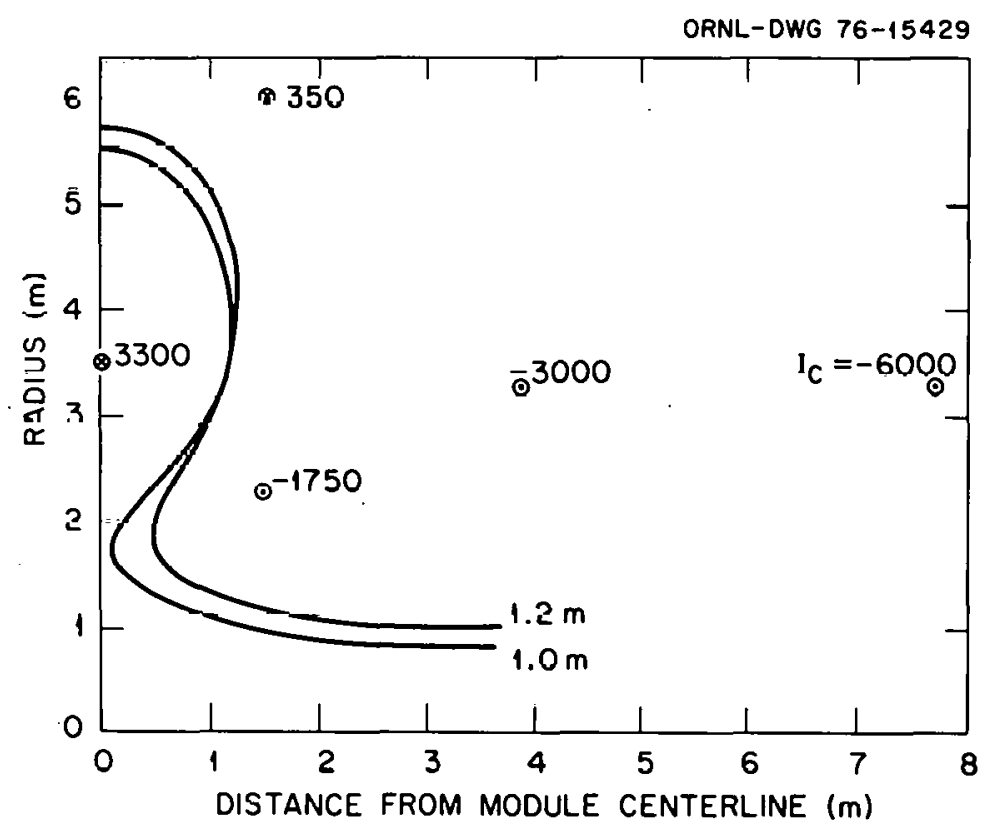

Fig. F-1. Field lines for EBTR-48 divertor. 
A "tickling" current of $350 \mathrm{~A}$ at $6 \mathrm{~m}$ radius is found to spread the field lines to about $0.5 \mathrm{~m}$. If this current is increased, the $1.2 \mathrm{~m}$ field line curls around this coil (see Fig. F-2, where a field null has formed near the "tickling coil"). By adjusting the currents and using more "tickling coils", one can spread the field further. By then shaping the divertor wall so that the field lines intersect it at an angle, the wall area needed to ensure that the plasma flux incident on the wall is within allowable limits can be attained.

A consideration of the mechanics of field shaping shows that for larger major radii reactors, divertor design will be easier, and more effective use will be made of the available volume. In general, however, the design of an EBT divertor does not seem to present any major difficulties.

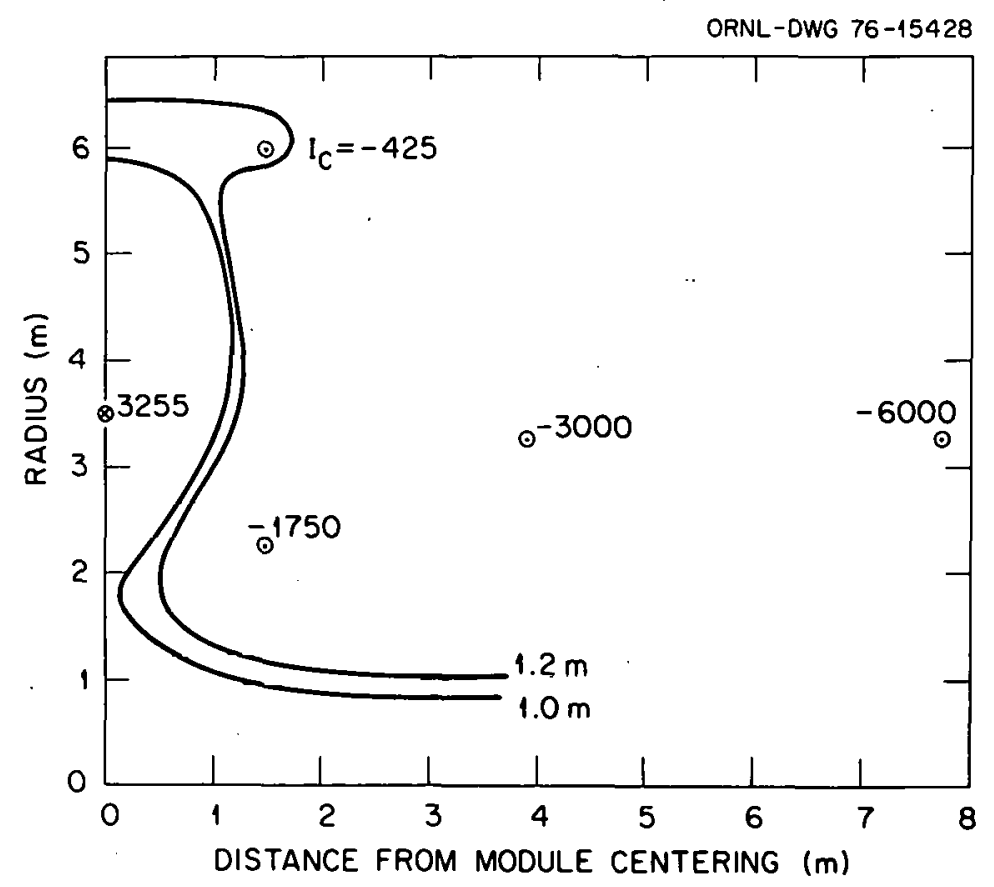

Fig. F-?. Field lines for EBTR-48 diverlur (with "tickling coil")

\section{DIVERTOR MECHANICAL DESIGN}

Figure F-3 shows the mechanical design of the EBTR-48 divertor. Four such divertors are located and equally spaced around the reactor and the design is such that any three of theil will be capable of handling the power recovery. The lithium cooled surface of each divertor is $597 \mathrm{~m}^{2}$, and for a total heat removal requirement of $\sim 800 \mathrm{MW}\left(\mathrm{th}\right.$ ) (the alpha particle power) the wall loading is $0.45 \mathrm{MW} / \mathrm{m}^{2}$ when three divertors are operating. This wall loading is well below the limits imposed by heat transfer to the liquid lithium.

A possible cryopump system for reactor pump down and reaction product removal is shown in Fig. F-4. Eight such systems (two for each divertor) will be sufficient to maintain a base pressure of $10^{-7}$ atm in the reactor with a cryopumping speed of $1.7 \mathrm{l} / \mathrm{cm}^{2}-\mathrm{s}$, which is well below currently available cryopumping capacities. This figure is based on a plasma density, $n$, of $1.5 \times 10^{20} \mathrm{~m}^{-3}$, a particle containment time, $\tau_{b}$, of $7.5 \mathrm{~s}$, and a total plasma volume, $v_{p}$, of $1184 \mathrm{~m}^{3}$. Then the particle flow rate, $\Gamma$, is 


$$
\Gamma=\frac{n V}{\tau_{b}}=2.37 \times 10^{22} / \mathrm{sec},
$$

and for eight cryopumping systems the speed, $s$, is

$$
s=\left(\frac{1}{r}\right)^{2} \frac{\Gamma}{8 p P f_{H e}} \text {, }
$$

where $A_{\mathrm{He}}$ is the area of the helium panels $\left(256 \mathrm{~m}^{2}\right), \rho$ is the gas density at 1 atill, $P$ is the base pressure, and $r$ is the reflection at the air and nitrogen-cooled panels (assumed to be 50\%).

The power required for nitrogen and helium refrigeration may be estimated from the energy deposited on each of the cryopanels (see Fig. F-4). Table F-1 summarizes the energy deposited.

TABLF, F-1

Encrgy Depos 1 toig in i.rymgralip is Deposited due to

Panel

\section{Particles
$(\mathrm{kW})$}

$$
\text { Air }
$$$$
0.010
$$

Nitrogen

0.011

Hel ium

\section{Blackbody (kW)}

25.8

1.21

0.005
25.8

(kW)

$\left(q_{\mathrm{N}_{2}}\right) 1.2$

$\left(q_{\mathrm{He}}^{2}\right) 0.008$

Assuming that the nitrogen and helium refrigerators operate at $\sim 10 \%$ of the Carnot efficiency, the total power required for the refrigerators is

$$
W_{R}=50 a_{N_{2}}+800 q_{H e}=68 \mathrm{~kW} \text { per cryopump system. }
$$

or a total of $544 \mathrm{~kW}$ for eight systems. 


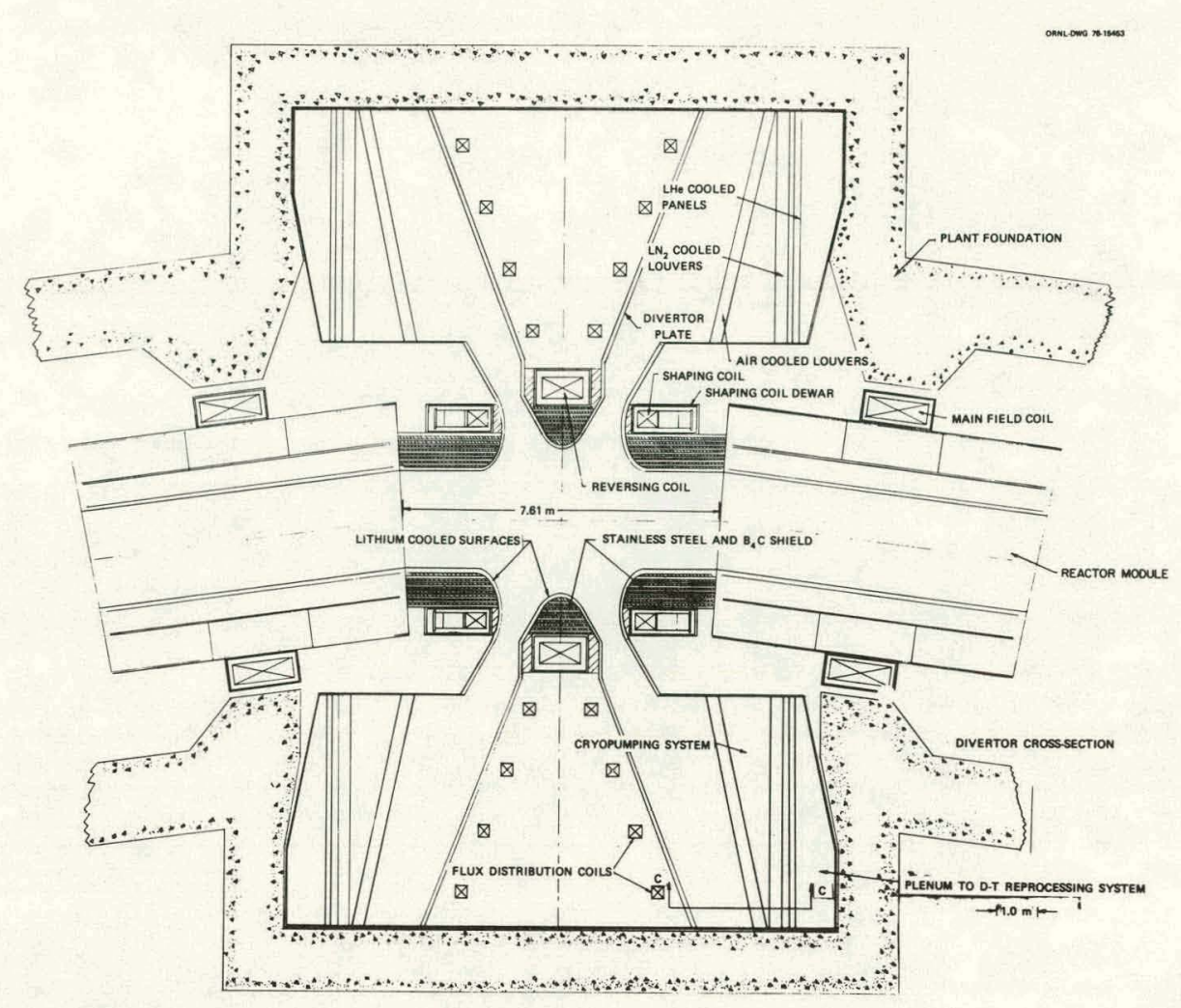

Fig. F-3. Divertor cross section.

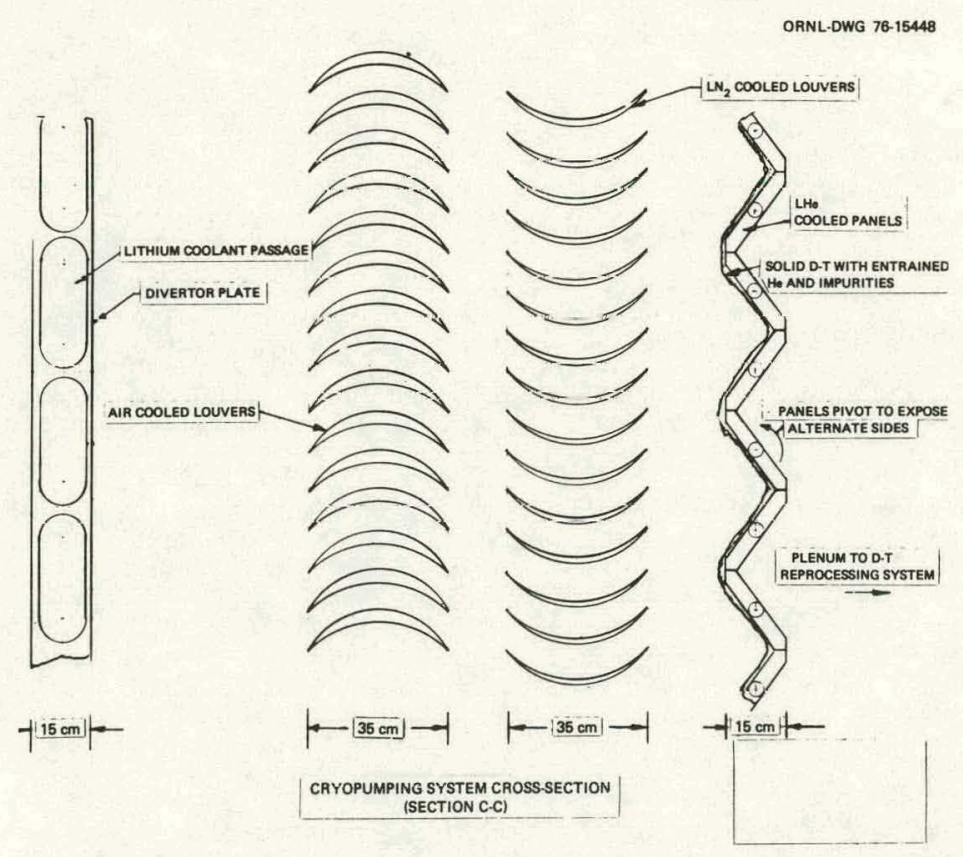

Fig, F-4, Cryopumping system cross section (Section $(-C)$. 


\section{INTRODUCTION}

Conceptual designs have been made of EBT reactors based on a modular concept with identical superconducting coils for a wide range of sizes. The major radius of the reactor was varied from a minimum of about $30 \mathrm{~m}$ to a maximum of about $120 \mathrm{~m}$ using the same coil design. The spacing between coils remained constant with the number of coils, of course, increasing directly with the major radius. The plasma radius and blanket and shield thicknesses also remained nearly constant for the various major radii considered.

In the reference design, the entire torus is enclosed in a concrete moat with an internal width of about $15 \mathrm{~m}$ and a height of about $18 \mathrm{~m}$, covered by modular concrete slabs. The concrete moat provides structural support for the torus and biological shielding to reduce radiation levels to 1 imits which are tolerable for personnel on a constant-exposure basis.

A11 maintenance is accomplished from overhead. The necessary remote maintenance equipment is mounted on top of the moat and can be moved over the top of the torus; a traveling gantry crane is also mounted on top of the moat and spans it. Power supplies for all the EBT reactor components, including the refrigeration systems for the cryogenic coils, and the tritium recovery processing systems are located outside the moat.

The torus is constructed of modules to make assembly and remote maintenance less difficult. The number of modules used can be equal to the number of superconducting coils or it can be twice the number of coils. In the latter case the modules which are located between the coils are not exactly identical to those located under the coils. When the number of moduTes is equal to the number of coils, the coils must be removed along with the blanket and shield when module maintenance is required. Alternatively, when there are twice as many modules as there are coils, the modules can be maintained without disturbing the coils. In this case a coil needs to be moved only if there is a failure in the coil itself.

Two reactor designs using fixed magnet concepts have been examined. Specifically, a machine with 48 coils and a 60-m major radius and a machine with 24 coils and a 24-m major radius have been considered. They represent two ends of a reasonable size range. As part of the studies it was determined that it is possible to design different-size devices which use the same coil and module design. The probable minimum size in this case is consistent with a $30-\mathrm{m}$ major radius. In the present discussion the EBTR-48 and EBTR-24 modules are not standard and their mirror ratios are not equal. Work on the standardized system is proceeding at present.

Sections 2 through 5 are a discussion of the mechanical and maintenance aspects of a reference device which is based on the fixed magnet concept, i.e., the number of modules is twice the number of coils. In this case nitrate salt coolant and stainless steel structure and shielding are used. Section 6 describes an alternate module concept using movable magnets, liquid lithium cooling, niobium structure, and a "heavy concrete" shield. Various other permutations of structure, shield, and coolant concepts are possible and will be considered when the overall system is optimized. The high aspect ratio configuration offers few constraints in this regard.

\section{FIXED MAGNET CONCEPT}

The first fixed magnet design studied had a $60-\mathrm{m}$ major radius, produced about $4000 \mathrm{MW}$ (th), exclusive of blanket multiplication, and had a neutron wall loading of about $1 \mathrm{MW} / \mathrm{m}^{2}$. A plan and a section 
elevation view of a portion of the torus are shown in Figs. G-1 through G-5. This machine includes 48 coils and has a mirror ratio of 1.8 . Two 24-coil systems with major radii of 24 and $30 \mathrm{~m}$ have also been studied. The more interesting of these is the 24-coil, 30-m system which uses the same coil and module design as the larger device. This design is particularly attractive if the neutron wall loading can be increased to $\sim 3 \mathrm{MW} / \mathrm{m}^{2}$ in which case the total thermal power of the 48-coil machine becomes unacceptably large. The layout for the EBTR-48 will be described, but the concepts apply in general to a wide range of device sizes.

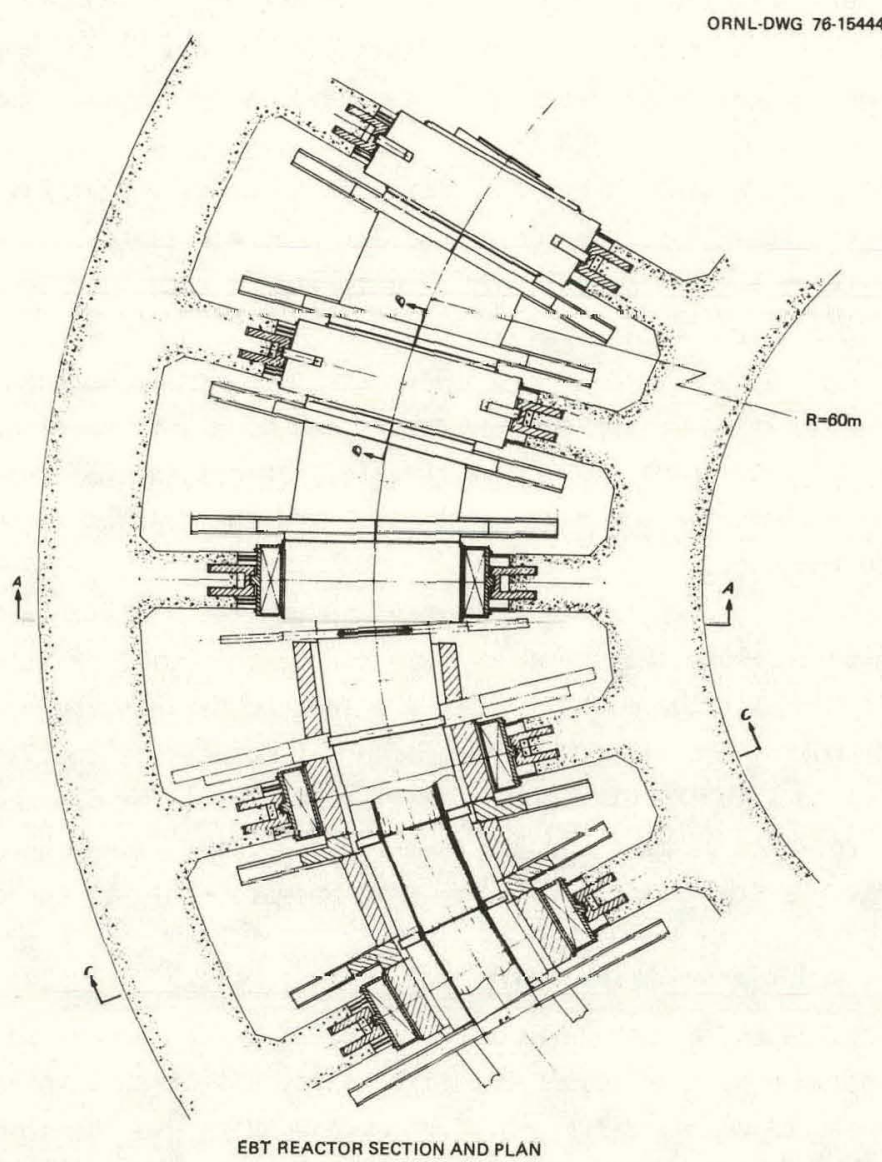

Fig. G-1. A plan and section elevation view.

The main torus consists of five concentric regions: 1) plasma, 2) first wal1, 3) blanket, 4) shield, and 5) superconducting coils to provide the mirror fields. These five regions are continuous around the torus but are composed of two types of cylindrical modules. One type is located under the coils and the other type is located between the coils. In addition to these basic modules, there are blanket and shield clamshell filler pieces which are put in place at the junctions between two modules after the joint between the module inner wall sections is completed.

With the torus joints located near the field coils, the modules between the coils present a large surface area which is available for vacuum ports, microwave and neutral particle injectors, and diagnostics. This area is available around the complete circumference of the module. The access gained here is distinctly superior to that in a low aspect ratio device. The injector design can also 
be modular and an increased heating power may be provided for a larger plant by adding more heating modules, such as injectors.

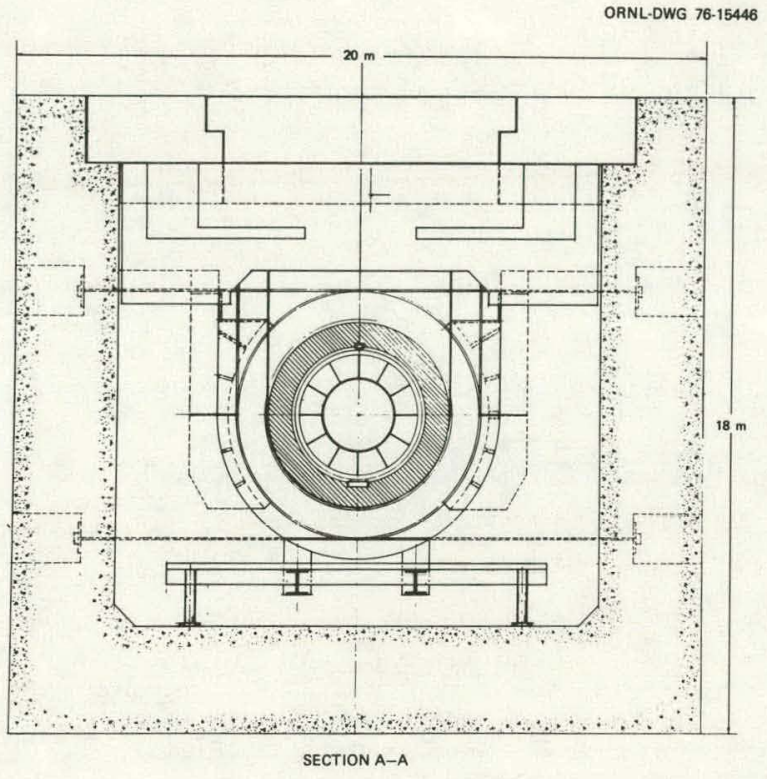

Fig. G-2. Section A-A of Fig. G-1.

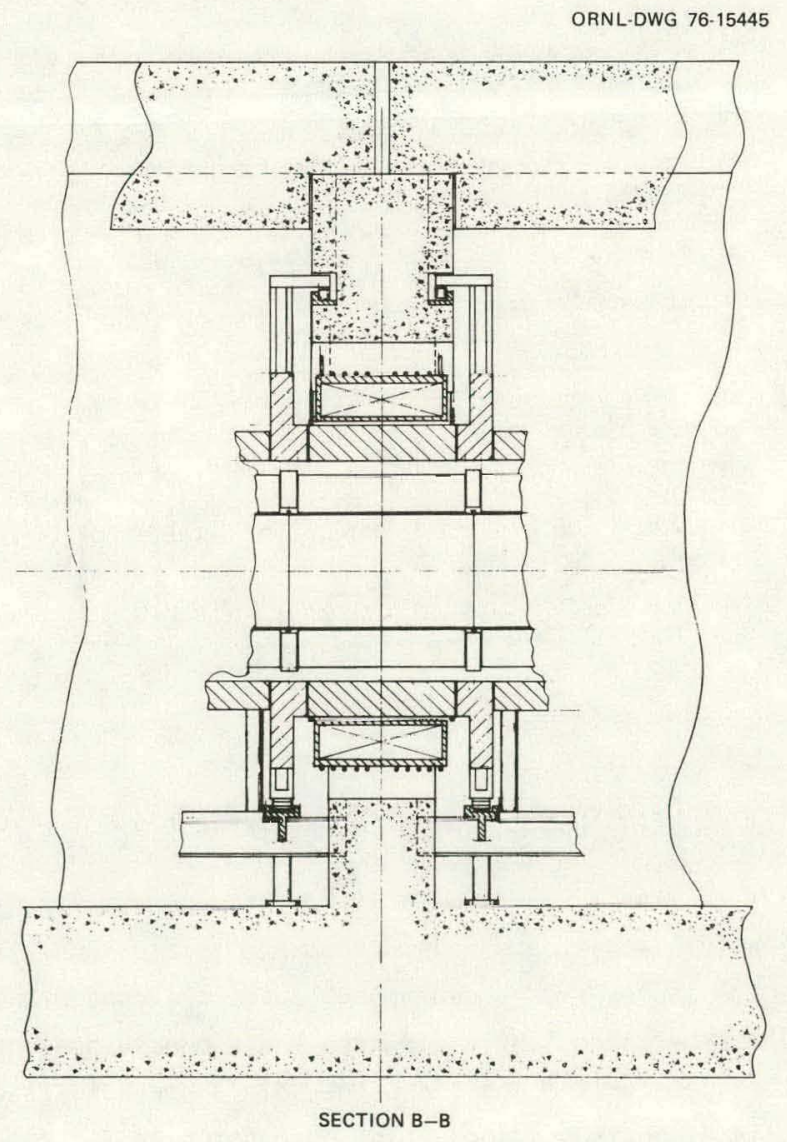

Fig. G-3. Section B-B of Fig. G-1. 


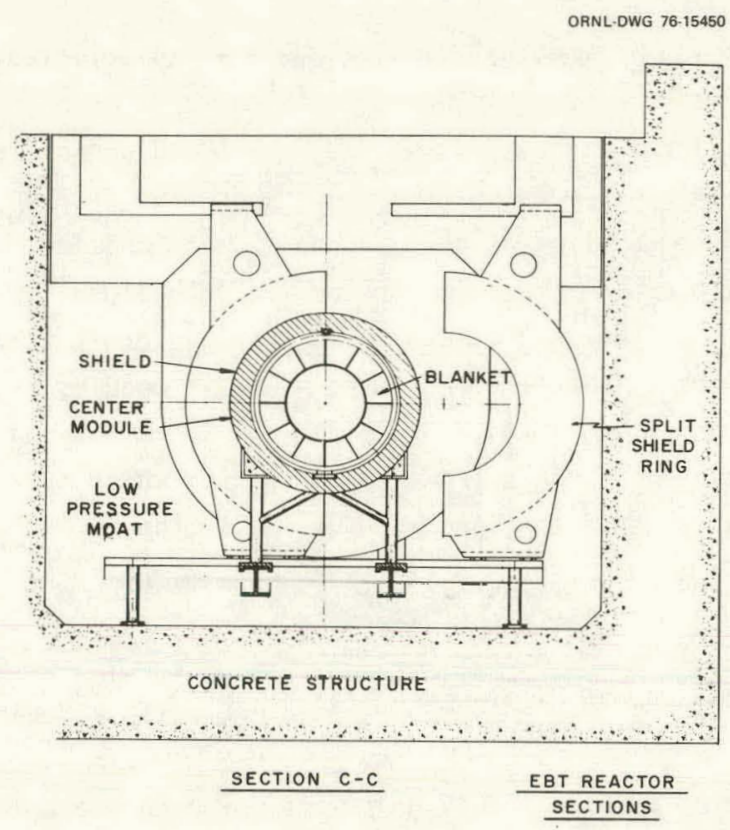

Fig. G-4. Section C-C of Fig. G-1.

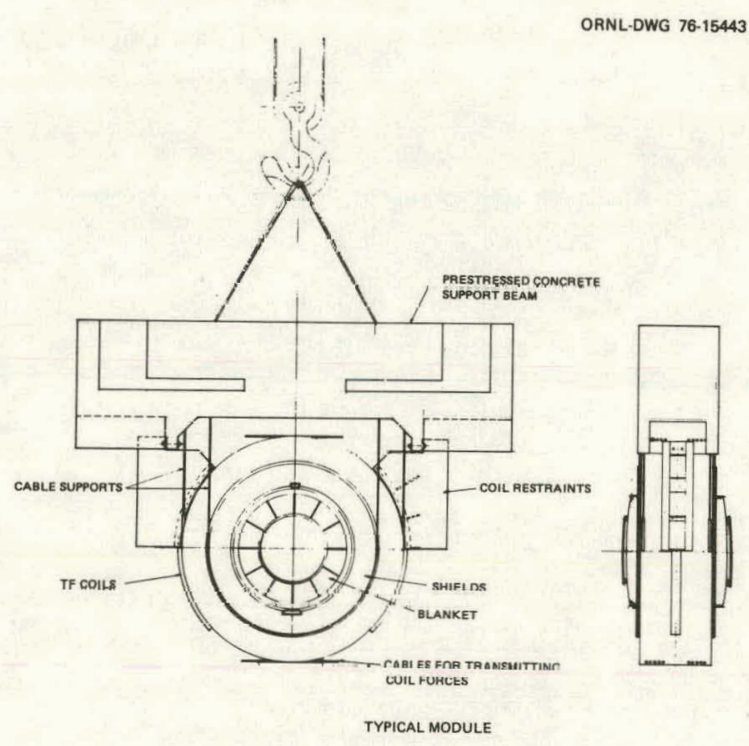

EBT REACTOR MODULE ASSEMBLY

Fig. G-5. Typical module assembly.

The modules of vacuum wall, blanket, and shield that are located under the coils are mounted as a unit and suspended by composite cables from a beam which spans the cuncrele moat on the bulkhead $\mathrm{cx}$ tensions from the walls. The superconducting coils have their own suspension cables and are mounted from the beam. The support cables for the coils and the shield are not attached to them, but pass under the cylindrical units in a continuous loop. The suspension cables for the coil contact the coil 
bobbin and are completely enclosed in the coil dewar. Where a cable leaves the bobbin at the tangent point, the dewars extend two feet up the cable and are sealed to it through a bellows. The blanket modules are supported from the inner radius of the shield by a pad and ballway linear bearings.

The modules between the coils are mounted from beams which run between the curved parts of the bulkheads rather than being suspended from an overhead beam. The shield is supported by four pedestals from the floor of the moat. The blanket rests on the shield on ballway linear bearings so that the blanket may be removed from the cylindrical shield after the shield has been lifted out of the torus. There are no forces other than gravity acting on these intercoil modules, so no further mechanical restraint is necessary.

In the case of the magnetic coils there are magnetic forces which must be counteracted. Under normal operating conditions, because of the large aspect ratio, the forces are essentially uniform and result mainly in a centering force. As in the case of the gravity loads, this force is also handled by cables which are looped around the coil under the coil dewar. There are two sets of these cables, one running through the inner wall of the moat and the other running through the outer wal1. The cables to the inner wall serve only to restrain the coil, so that the centering load cables can be preloaded to make the load distribution uniform.

The cable suspension and restraining method has two significant advantages over rigid mechanical mounts. The flexible cables conform to irregularities in surface contours and therefore ensure equal stress on the various parts of the coil bobbin. There is no danger of concentrations of stress resulting from point contact. The other advantage has two aspects. First, the cable supports are located under the dewars and will create lower thermal losses than those which would result if rigid supports were used. Second, as the coils are cooled down from ambient conditions, the cables will follow the dimensional changes so that support and restraint characteristics are unchanged at cryogenic temperatures. This could not be accomplished satisfactorily using a rigid support scheme.

It is evident that cables can be used successfully for vertical and radial loads, and these are the only loads imposed during normal operation. However, if one or more coils fail while the others remain excited, there will be large lateral loads which must be constrained. It may be possible to protect against this by electrically unloading all coils in a controlled manner if one coil fails. Methods for doing this are discussed in Appendix E. In case this protection scheme still permits large forces, gross movement of a coil must be prevented. At the same time, rigid mechanical restraints which would present thermal leakage paths are undesirable.

Provision has been made to install "bumpers" in the concrete bulkheads adjacent to the coils. Recesses in these bulkheads contain a stainless steel restraint structure which has a keyway that loosely fits over the key in the coil. The key is enclosed in a dewar. These restraint fixtures cover a $45^{\circ}$ segment of the coil above and below the equator on each side of the coil. Normally there is no contact between these fixtures and the coil and thus no heat leak path is established. In the event of a coil failure (current decay is assumed to take $2 \mathrm{sec}$ ), the out-of-plane forces cause the coil to "lean" into these bumpers. The dewar at the pressure point is crushed, but gross movement of the coil is prevented.

The assembly of the torus is straightforward. First, the coil modules are suspended from the concrete mounting beam while the beam is on an assembly station jig. The shield is then inserted in the bore of the coil and the suspension cables are attached to the beam. The blanket module is then inserted into the shicld and the first wall cylinder is inserted into the blanket. The entire assembly is transported to the moat and installed in place. All coil modules are located in place in the moat.

The next step is to mount a center shield module on the assembly jig and install the center blanket cylinder in the shield and the first wall on the blanket. This assembly is then inserted into the torus 
between two coil modules. The process is repeated until the full torus is complete. The individual first wall modules must next be joined together either by welding or by mechanical design. The final decision on the closure method has not been determined. The $180^{\circ}$ blanket filler pieces and the $180^{\circ}$ shield filler pieces next are installed over the joints between first-wall cylinders.

After the torus is completed in the moat, the installation is completed by first attaching all coolant lines and manifolds. When all interconnecting plumbing has been completed, the various penetrations, i.e., microwave, beam lines, diagnostics, etc. are attached and the closing concrete shielding slabs are installed.

It may be desirable to run the entire moat at a pressure of about $10^{-5}$ torr. This might permit the first wall sections to be mechanically clamped rather than welded together, and would also simplify connection of penetrations. If this option is followed, a metal skin will be welded in segments over the concrete roof slabs to provide a vacuum enclosure.

\section{BLANKET AND HENT REMOVAL}

Heat removal is accomplished in three separate components of the torus: 1) the first wall, 2) the blanket, and 3) the shield. As a conservative starting point for this design, it was decided to study a system in which provision was macie to control impurities through some sort of plasma surface control but to neglect the possibility of energy removal through divertors. In this case the first wall is exposed for the most part to non-neutron energy from the plasma plus a small portion of the neutron energy which is deposited in the material and coolant of the first wall. The energy delivered to the first wal1 is about $20 \%$ of the fusion energy produced in the plasma and results in a high heat flux on the first wall. Since nearly all the energy is absorbed on the surface, it presents a difficult thermal stress problem. A completely satisfactory design for this first wall has not been developed. Some requirements for it are clear. The wall must 1) form a low pressure boundary region for the plasma, 2) be as thin as possible to limit the number of neutrons absorbed or moderated, 3) have all adequate coolant flow consistent with the high heat transfer requirements, 4) operate in a low pressure environment, and 5) operate at relatively low temperatures ( $v 400^{n} \mathrm{c}$ ) to prolong life.

The blanket is located immediately behind the first wall. Its function is lu absurb the highest practical percentage of the plasma energy which is not deposited in the first wa11. $1 \mathrm{l}$ must provide for the conversion of this energy to heat at a usable temperature and it must breed tritium to fuel the reactor. The blanket consists of stainless steel compartments which are held in a structural framework to form an articulated cylinder between the vacuum first wall (1iner) and the shield. Inside these compartments are I1thrum metal, a canred yraphile ieflector, stainless stcel plates to ahsnrh gamma rays, and coolant tubes.

A schematic drawing of the blanket showing its location, composition, and representative dimensions is shnww in Fig. $f_{7}-6$. In this schematic the coolant tubes are not shown; they are continuous parallel tuhes which are located in the lithium regions and which provide cooling for the entire blanket. The coolant for both the first wall and the blanket is assumed to be a eutectic of nitrates of sodium and potassium. The coolant loop for the first wall is separate from the coolant loop for the blanket.

Coolant enters the first wall cooling circuit at $260^{\circ} \mathrm{C}$ and exits from it at about $310^{\circ} \mathrm{C}$. Exact heat transfer calculations cannot be made because the actual design of this wall is still undetermined. Based on preliminary configurations, the temperature drop across the fillil is round to be on the order of $55^{\circ} \mathrm{C}$ and the temperature drop across the metal wall is about $35^{\circ} \mathrm{C}$. It is probable that the hot-spot metal temperature of this wall will be about $400^{\circ} \mathrm{C}$. This value is consistent with those which give maximum lifetime expectancies. These figures are based on neutron wall loadings of $3-3.5 \mathrm{MW} / \mathrm{m}^{2}$. 


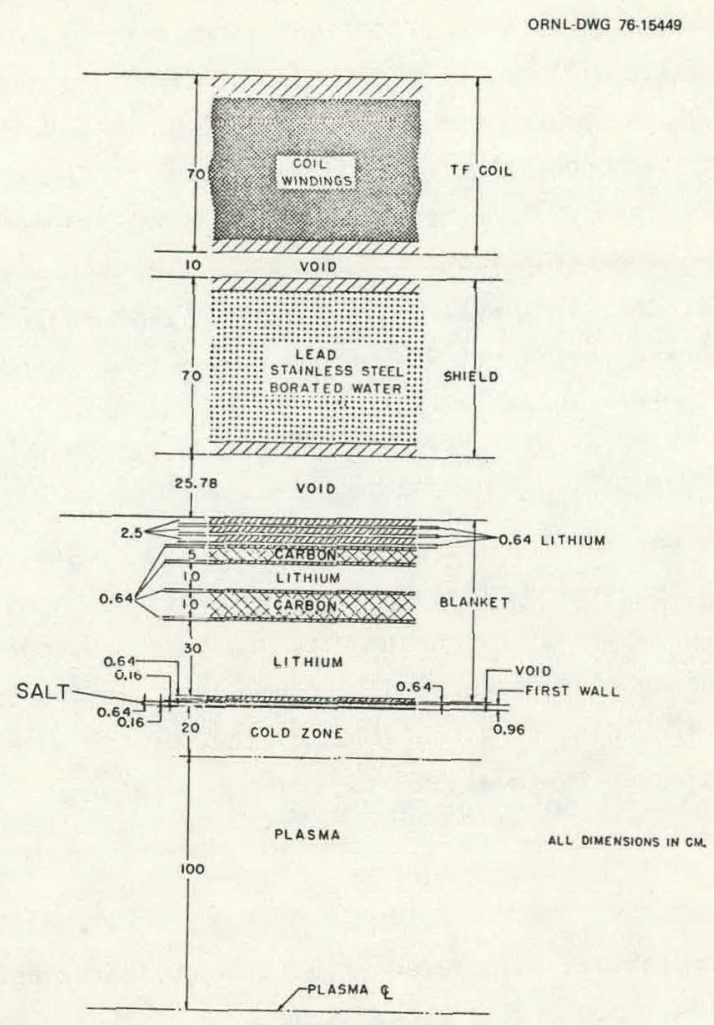

Fig. G-6. EBTR Blanket.

The blanket is cooled by a second loop which contains the same coolant as used in the first wall. In the blanket the coolant enters at $260^{\circ} \mathrm{C}$, flows uniformly down the sides of the double walled blanket compartments, enters a manifold, and flows into the parallel array of coolant tubes. The exit temperature of the blankct coolant is $480^{\circ} \mathrm{C}$. Final details of the design of the blanket have not been established. It can be similar to the construction of a tokamak blanket where a considerable amount of design detail has been developed.

The first wall coolant loop and the blanket coolant loop will transfer heat to a common intermediate coolant loop which contains a molten salt of the same composition as the two primary loops. This intermediate loop transfers the fusion energy to the steam generating system. The intermediate loop is used to isolate the high-pressure steam piping from the reactor and to isolate the radioactive primary loop from the steam system.

The use of the nitrate eutectic salt loops results in a low pressure coolant for the reactor. This allows the use of thin metal structures throughout the system. Also, the heat transfer characteristics of the salt are such that the pumping power is quite low, e.g., less than $1 \%$ of the thermal power produced. Ihe pressures, flow rates, and pressure drops are lower for the molten salt coolants than for any other coolants which were studied.

By using double walls for the blanket compartments and by introducing the $\operatorname{cool}\left(260^{\circ} \mathrm{C}\right)$ salt between the walls, the temperature of the container metal is about $315^{\circ} \mathrm{C}$. In the design of the blanket, modified 316 cold-worked stainless steel is used for the structural material. It is possible to use a different material for the first wall if this proves to be desirable, since the tirst wall is a completely autonomous structure. 
The present first wa11, blanket, and shield configurations make use of cylindrical modules. This geometry assumes that shaped walls will not be required, but it may be necessary to shape these elements so that they conform roughly to the plasma shape. In this case the modules between the coils will have a larger radius than those located under the coils. This design can be accomplished without altering the basic overall design of the torus. A decision on shaping these elements will follow further evaluation of the plasma containment properties.

The nuclear performance of the first wall and blanket has been evaluated using a one-dimensional analysis. The calculations showed that about $98 \%$ of the fusion power produced is deposited in these two components. In addition to the favorable heat deposition characteristics, a breeding ratio of about 1.35 was attained. The breeding calculation did not include penetrations which may occupy about $5 \%$ of the blanket surface area.

While the blanket absorbs most of the energy produced by the plasma, some additional shielding must be provided to protect the superconducting coils. The shield is a mixture of stainless steel, lead, and borated water. Ihe one-dimensional neutronics analysis showed that the shleld prulecls lhe magnets adequately and that the neutron damage and heating levels in the superconductors are within tolerable limits. The shield is cooled by circulation of the borated water through a heat exchanger; the maximum temperature of the water is about $82^{\circ} \mathrm{C}$.

\section{REACTOR MAINTENANCE}

One of the more difficult problems associated with thermonuclear reactors is that of maintenance. This is particularly true of low aspect ratio machines because of their inherent inaccessibility. This particular problem is alleviated in the EBT reactor chiefly because of its large aspect ratio and the absence of a poloidal field system. While the maintenance problem will probably be eased in the case of a high aspect ratio device such as EBTR, it nevertheless presents a formidable challenge.

After a short period of operation, the high energy neutrons produced will activate the internal components of the machine to high levels. The blanket and shield will protect the coils from the neutron flux, but the penetrations for pumping and injection will present addiliund leakage paths which may well result in a torus region which is too radioactive to permit hands-on maintenance. The maintenance policy, therefore, is to provide a totally remote maintenance capability for any procedure which must be accomplished within the confines of the moat.

This requirement influences the mechanical design of the entire reactor. There are certain operations that will be required on a periodic basis. Replacement of the inner wall and blanket at least one or more times during the life of the plant is expecled and is one of the major maintcnance tasks. In addition to the anticipated component replacement and repair activities, the entire system inside the moat must be designed so that unexpected failures can be dealt with efficiently.

A11 maintenance is accomplished using the overhead access described in Sect. 1. Articulated conr.rete shielding blocks span the moat, and removal of these blocks from any location around the circumference of the torus provides access to the equipment below. One or more gantry cranes are mounted on top of the moat and service the reactor. In order to provide for maximum flexibility, at least two cranes should be installed. These cranes will be used to lift major components and move them to a service cubicle which will also be located on top of the moat. The service cubicle will be mobile so that it can move around the full circumference of the top of the moat. The crarles Illdy diso be used to lower special maintenance tools and equipment into the moat.

Tools have not yet been designed to perform the maintenance functions which are expected to be necessary. Certain types of operations that are known to be required have been identified. These are: 
1) transporting and positioning large components, 2) remote cutting and welding, 3) mechanical clamping and bolting, 4) continuous viewing and inspecting, 5) making and breaking coolant and electrical connections, 6) provision for special instrumentation, and 7) procedures for waste disposal.

Many of these operations must be performed inside the concrete enclosure. For example, electrical and coolant connections must be made and broken inside the moat. Cranes must remove the access plugs and lower the special tools and viewing equipment into position to accomplish these operations. A fundamental philosophy for maintenance is that each operation will be accomplished using visual control. The size and complexity of the reactor is such that preprogrammed operations probably cannot be carried out with sufficient accuracy. Dependable three-dimensional remote viewing is essential for satisfactory maintenance. There are many preparatory operations which will have to be performed prior to replacing an internal wall or blanket module and these can best be controlled visualiy.

Any unscheduled repair will be time-consuming. The complete replacement of the major internal components of the reactor probably will require months. The specific maintenance operation will have to be carefully planned, and the most reliable equipment provided to minimize the downtime associated with a repair, which vitally will affect the plant economics. Duplication of all maintenance equipment is almost certainly a requirement. It is likely that major maintenance such as replacement of the first wall and blanket will be accomplished simultaneously at more than one location around the torus.

When a section of the torus has been isolated by breaking all connections (including the two circumferential joints between adjacent modules), the module will be lifted into the maintenance cubicle where the detailed repairs will be made. The repaired unit will be returned to the torus and the radioactive components will be cut up, compacted, and transported to a hot storage area within the controlled area of the building. This storage area will most likely be a pool of water in which the radioactive material will be stored until it cools sufficiently to permit removal and transport to a permanent storage area. The maintenance problem cannot be overemphasized. If everything associated with the reactor works satisfactorily and unanticipated failures are infrequent, it is certain that radiation damage will necessitate periodic replacement of some components. The downtime necessary for this replacement represents a loss of production of the plant and influences greatly the cost of the power produced.

\section{HEAT LOSSES}

There are two types of heat losses that are important to a power-producing, superconducting thermonuclear device: heat loss from the power conversion system (blanket) and heat loss from the cryogenic system (coils). The latter is probably the more important, but the blanket losses cannot be neglected, since the large surface area of the blanket represents a potential heat loss source unless good thermal insulation is provided. This heat loss can probably be tolerated by locating the torus in an evacuated environment and by providing reflective insulation to cover the exposed blanket surfaces.

Control of the heat loss from the cryogenic coils is a more difficult problem. This loss is especially important since each watt of heat removed at $4.2^{\circ} \mathrm{K}$ requires between 500 and $1000 \mathrm{~W}$ of refrigeration power. It is necessary to enclose the coils in an efficient dewar, and this requirement imposes design problems on the mechanical restraint system for the coils. The structure which contacts the coils must operate at $4.2^{\circ} \mathrm{K}$ and conductive heat paths to structures which operate at ambient temperature must be minimized.

In the current EBT reactor design, the composite cable suspension and restraint system minimizes the heat leakage from the coils. Belluws-sealed dewars provide the transition for each of these cables where they leave the coils. Each dewar, of course, encloses an entire coil. 
The heat loss is reduced in two ways by using composite fiber cables. These composite cables have a very high allowable working stress so that the area of the tension member is smaller than that required for steel cables. The thermal conductivity of the graphite fiber is increased relative to that of steel so that a means of introducing a high thermal resistance is necessary. This will be accomplished by introducing a strain insulator similar to that used on electrical transmission guy wires for electrial insulation purposes. These strain insulators are loaded in compression and can be made to transmit the tension loads between the two cable lengths through a low thermal conductance path.

If the entire temperature drop is assumed (pessimistically) to take place in this insulator, and each insulator is $15 \mathrm{~cm}$ long, the heat loss per coil will be about $320 \mathrm{~W}$. There are 28 insulators per coil. For a 24-coil reactor the total heat loss will be about $7.7 \mathrm{~kW}$. Assuming a refrigeration conversion factor of $500 \mathrm{~W} / 1 \mathrm{~W}$, the electrical load for cooling all the coils will be about 4 MW. These calrulations were made assuming the use of a high-density polyethylene insulation material. For the heat losses through the dewar, assuming that the outside of the dewar is maintained at ambient temperature, the loss per coil will be about $80 \mathrm{~W}$. For 24 coils, the total loss is about $2 \mathrm{~kW}$, Ihe equivalenl electrical load here is $1 \mathrm{MW}$. Thus, for the entire 24-cull machime, the requircd cryogonic cooling load is about $5 \mathrm{MW}$. This does not account for losses from the electrical terminals, but these losses are not expected to be large.

Since the coils are almost identicā in EBTR-24 and EBTR-48, the refrigeration load will be nearly proportional to the number of coils. On this basis, the 48-coil machine would require about $10 \mathrm{MW}$ of refrigeration power for the cryogenic system. The thermal power produced in the 48 -coil reactor is $4000 \mathrm{MW}$, so the refrigeration load is only about $0.25 \%$ of the thermal output.

These heat loads were calculated assuming a $4.20 \mathrm{~K}$ helium system and an ambient temperature of about $32^{\circ} \mathrm{C}$. The total refrigeration load could be reduced if a buffer region of $\mathrm{N}_{2}\left(77^{\circ} \mathrm{K}\right)$ is used between the helium system and the ambient. Other analyses have indicated that an intermediate nitrogen region results in a lower overall refrigeration load. However, this seems unnecessary in the present EBTR.

\section{MOVABLE MAGNET CONCEPT}

The particular movable magnet design presented here, shown in Fig. G-7, differs from the fixed magnet reference design in four significant ways: 1) the design is a set of selfcontained blanket-shield-coil modules individually removable for maintenance; 2) the choice of a heavy concrete ( $40 \%$ iron shot by volume) magnet shield, 3 ) the usc of natural 1iquid 1ithium as both conlant and hreeding material; and 4) the use of niobium as first wall and as structural material in the first wall zone. The first two of these design options could be implemented immediately, the third and fourth would require further development. Thus this design has been curlsidered as a somcwhat advanced, alternate version of the reference design. It is important to note that these particular design choices are nearly independent of each other and could be combined in many ways with those of the reference design. This is convincing evidence of the flexibility aftorded by high dspecl ratio sys tems.

Natural lithium is proposed as the coolant because of its superior heat transfer and neutronic. characteristics. In addition, the use of one material as both the coolant and breeding material simplifies the heat exchanger loop design, reduces the number of heat exchangers necessary, and thereby reduces the probability of leaks. The major disadvantage in the use of lithium is the pressure drop which results from MHD effects when the fluid flows perpendicular to the magnetic field. These effects require special attention in the design of the lithium inlet and outlet plena. The calculations 
performed have shown that, for a plenum thickness of $15 \mathrm{~cm}$ and height of $3.70 \mathrm{~m}$, the pressure drop in either an inlet or outlet plenum is about $800 \mathrm{kPa}(120 \mathrm{psi})$. The ideal pump work required is $150 \mathrm{~kW} / \mathrm{plenum}$.

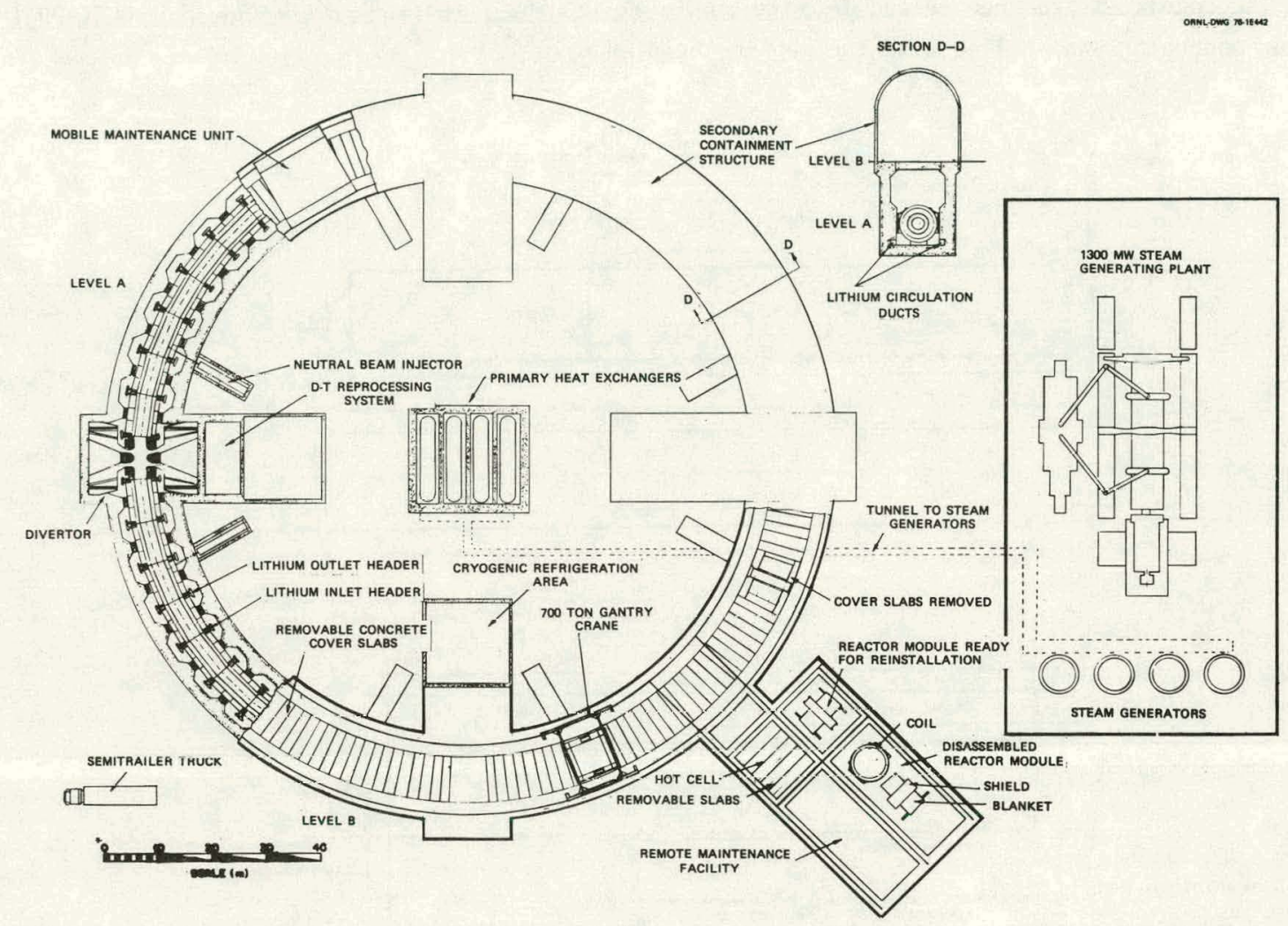

Fig. G-7. EBTR plan view.

The main problem posed by the MHD pressure drop is not so much the power consumed (1ess than $1 \%$ of the module thermal output at $50 \%$ pump efficiency) but rather the stress imposed on the inlet plenum and the first wall by the outlet plenum pressure drop. Electromagnetic pumps are proposed for use in the inlet and outlet plena to solve this problem. These pumps would consist of electrodes with surfaces which are parallel to the magnetic field lines and approximately radial from the plasma centerline. The electric current would flow azimuthally around the plasma centerline through the lithium coolant. In this way the pressure drop and the pumping action both occur at the same location and relieve the stress on the plena and the first wall. The coolant which flows along the first wall is not exactly parallel to the magnelic field lines and thus there will be residual pressure drop. The flow channels in the first wall have been designed to be loaded in tension and anchored to a supporting wall. This is shown in Figs. G-8 and G-9. The detail of the first wall root shows that the first wall is composed of $\mathrm{Nb}-1 \% \mathrm{Zr}$ ducts formed into $\sim 20-\mathrm{cm} \times 20-\mathrm{cm}$ channels. The channels are welded at the root ends in a vacuum or inert atmosphere before they are inserted into slots in the supporting wall. The channels are put in tension by this loading arrangement and they serve as stiffening ribs for the supporting 
wal1. The lithium inlet and outlet plena have small gaps leading into the breeding blanket. This gap allows sufficient flow of lithium through the region for heat removal. It also provides clearance for the assembly and disassembly of the blanket. The unit is assembled by first welding the first wall channels together, then inserting the first wall assembly into the supporting wall which includes the plenum extrusion plate, and finally welding the lithium blanket endplates to the first and supporting walls. The endplates are then welded into the module at a point immediately in front of the graphite reflector-moderator and in line with the gap in the plenum wall.

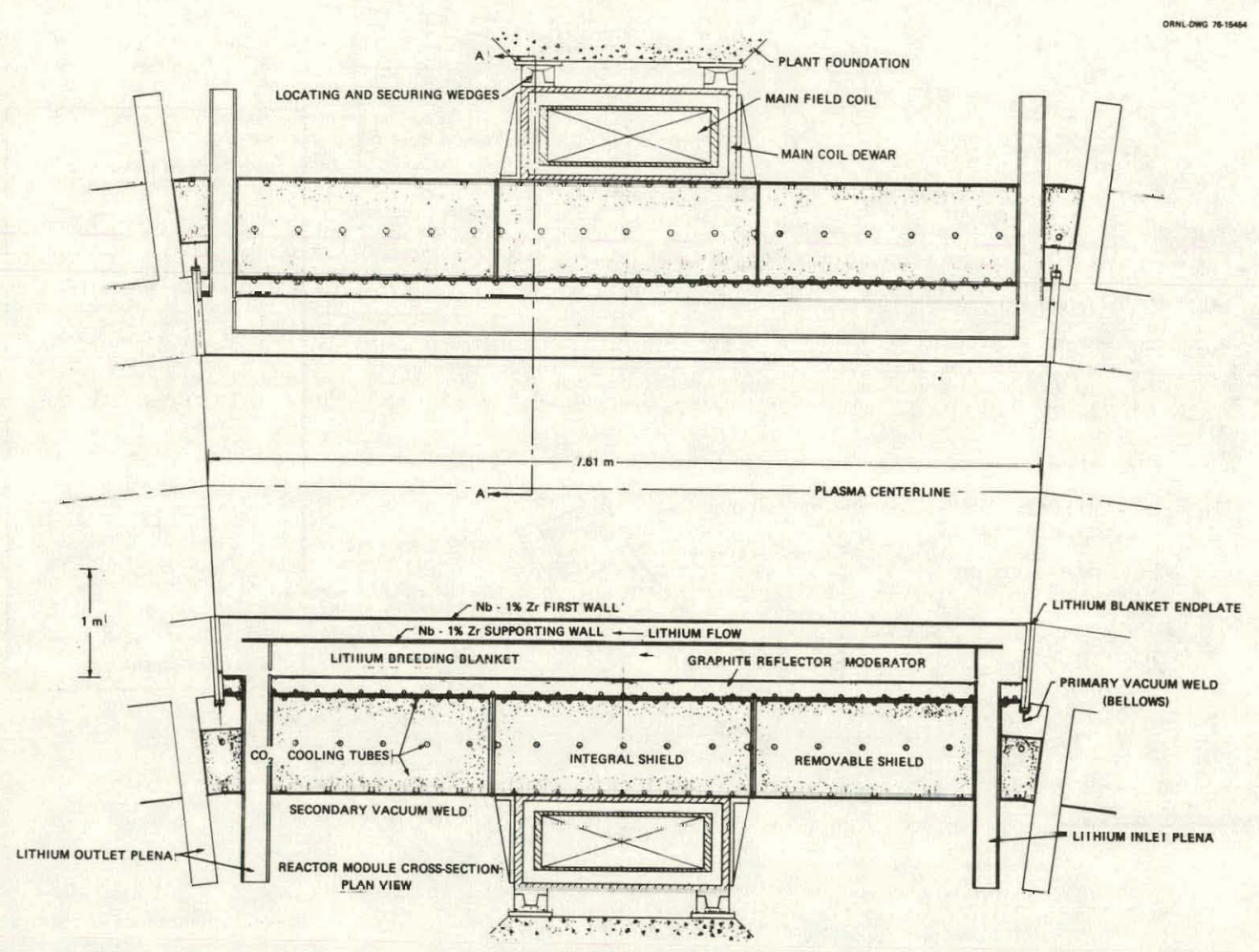

Fig. G-8. Reactor module cross-section plan view.

When the first wa11 has been exposed to the maximum tolerable fluence, the entire module will be removed. To accomplish this, the endplates are cut and the first and supporting wall assemblies are removed and replaced by a new unit.

Another variation in this design is the provision for magnet coils which can be removed along with the blanket and shield when maintenance becomes necessary. The basic philosophy is that module maintenance should cause as little downtime for the entire reactor as possible. Therefore, the operation of removing a failed module and installing a new or repaired module must be as simple as possible.

A second advantage of the movable module approach is that work on a failed coil or a failed shield cooling system can be accomplished in specially equipped hot cells. The principal disadvantages in removing the coil and shield during maintenance are the difficulties associated with handling the large 
weights, the possibility of damaging a magnet during the operation, and the complexities of the electrical and cooling connections.

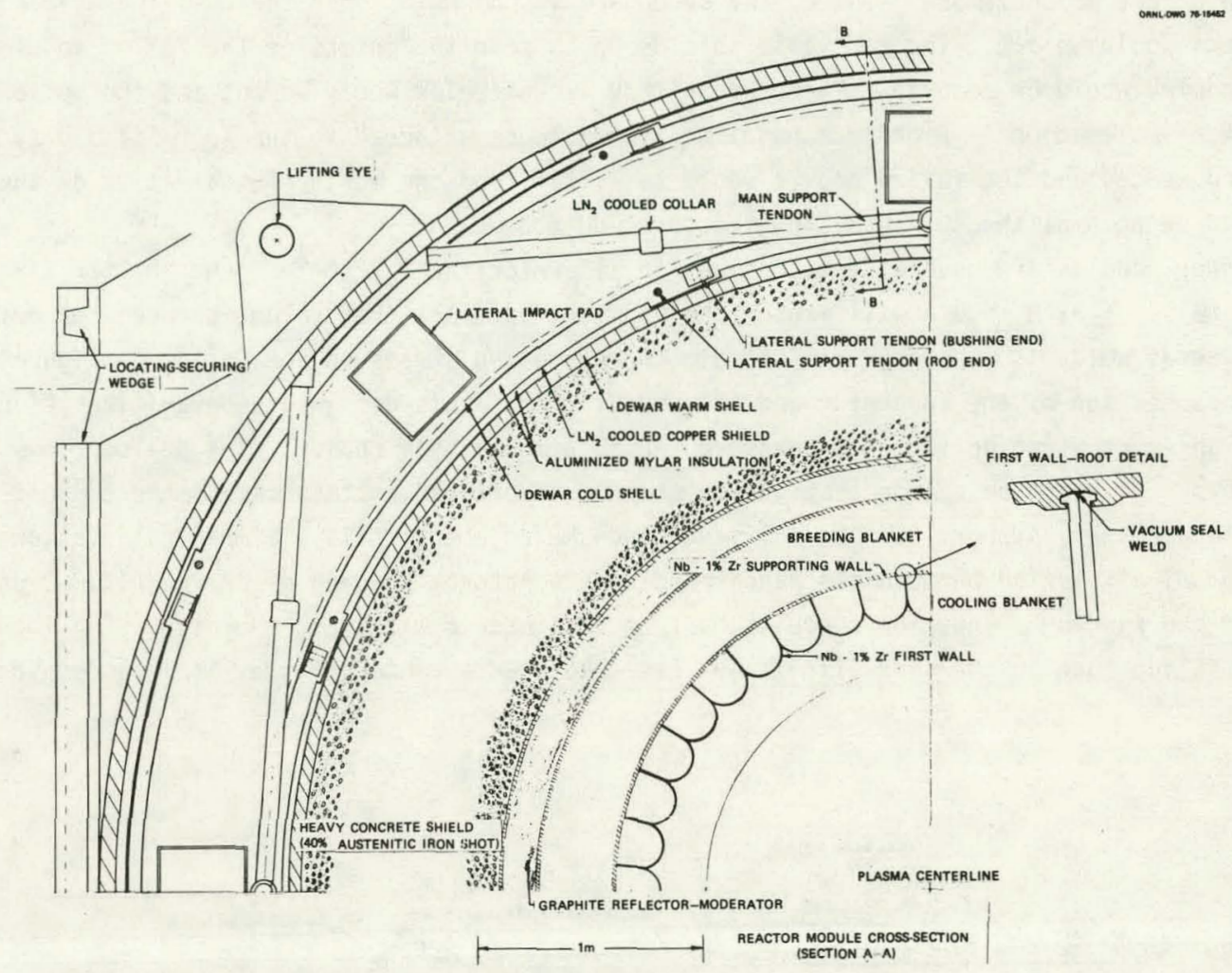

Fig. G-9. Reactor module cross-section (Section A-A).

The magnet, integral shield, andblanket assembly are estimated to weigh $\sim 600$ tons. The removable shield will weigh about 450 tons. While these weights are large, they are well within the current technology, all equipment specitically designed for the movement of these modules could provide the precision required.

As shown in Figs. G-8 and G-9, the main coil dewar has been designed to support and protect the coil windings from magnetically imposed lateral shock loads which could occur due to the quenching of an adjacent coil. Since these loads ( $\sim 54 \mathrm{MN}$ ) far exceed the weight of the windings ( $\sim 1.2 \mathrm{MN}$ ), the danger of damage to the coils due to a lateral shock during coil movement seems rather small considering that the design shock load is $45 \mathrm{~g}$. In addition, the dewar has been designed to withstand a force from any direction in the plane of the coil of $8.4 \mathrm{MN}$. This is the approximate centering force experienced by a coil during normal operation. Thus, the coil can withstand at least a $4 \mathrm{~g} l o a d i n g$ in the plane of the coil during movement.

As shown in Figs. G-8 and G-9, the reactor module is positioned and secured to the plant foundation by wedges which are $10 \mathrm{~cm}$ deep and $\sim 3.5 \mathrm{~m}$ long. The wedges are attached to the corners of the dewar warm shell. The system of wedges affords the needed accuracy in the remote positioning of the reactor module. This design avoids any needless stress on blankel and first wall by supporting the module near the source of its maximum loads. 
The remote disassembly procedure for a failed module would be as follows: first, the liquid helium and electrical supplies to the dewar would be disconnected. Next, the lithium inlet and outlet plena would be cut or unclamped. Third, the secondary vacuum welds near the coil and adjacent to the lithium plena would be cut. The removable shields which span the points of the failed module to the adjacent modules would be removed. Then the primary vacuum welds would be cut and the bellows would be folded back a small amount. Finally a handling jig would be attached to the four lifting eyes above the locating wedges and the failed module would be lifted from the moat. Installation of the replacement module would be accomplished by reversing this procedure.

The power modules are connected by bellows to allow for the $2.2 \mathrm{~cm}$ that the $\mathrm{Nb}-1 \% \mathrm{Zr}$ first and supporting walls of each module will expand during start-up. The free volume between the primary and secondary vacuum welds is evacuated to $10^{-3} \mathrm{~atm}$ to reduce the stress on the bellows. Figure G-10 shows a cross section of the conductor and its conduit. For this design a supercritical, forced-flow rnnling arrangement using helium at a pressure $>2.53 \mathrm{~atm}$ has been chosen. The helium flows parallel to the filaments. In addition to the stability improvements offered in this case, ${ }^{1}$ the conduit structure provides the necessary support for the filaments and the adjacent coils. Simple calculaliums indicate that the mutual attraction between the pancakes causes a lateral loading of $82 \mathrm{MPa}(11,800 \mathrm{psi})$ at the midplane of the wiriding. Thus the conduits must be designed to withstand these crushing loads. The critical buckling load on the sidewalls is not exceeded when a center divider is included in the conduit.

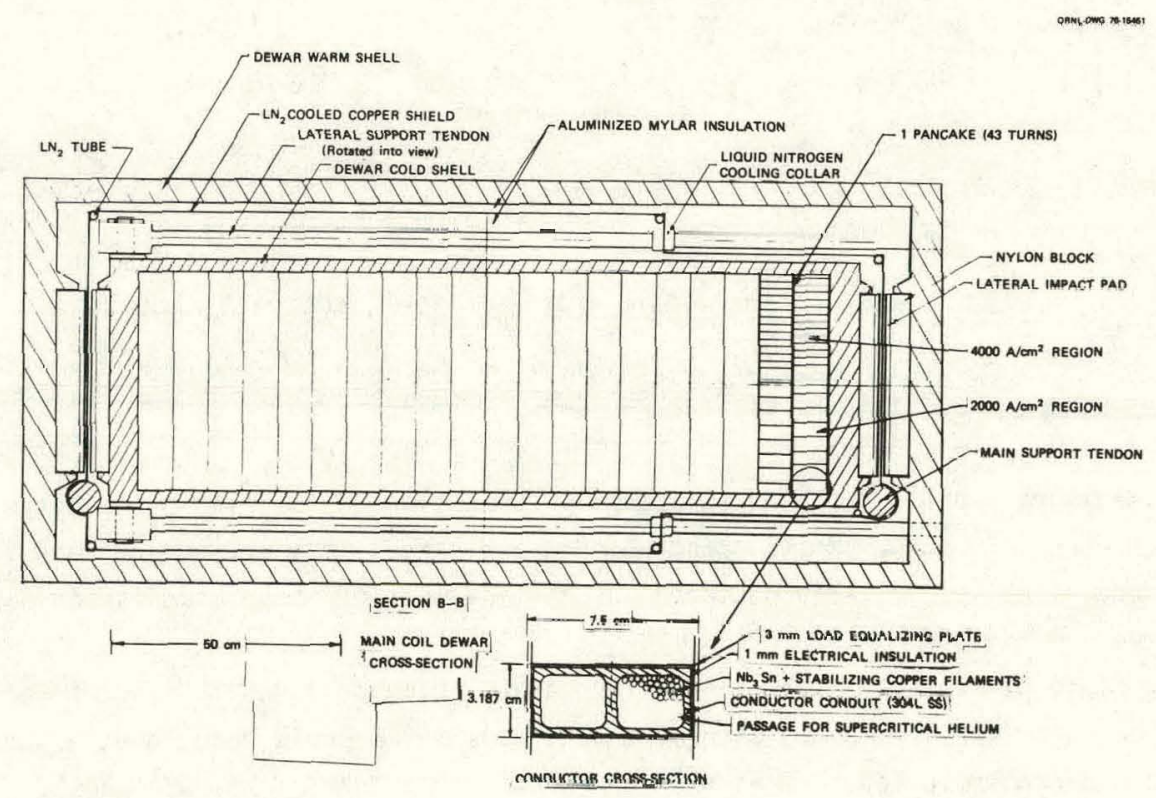

Fig. G-10. Main coil dewar cross-section.

One problem that may prove significant is the lateral heat transfer between havles of the paricakes or between adjacent pancakes. If the electrical insulation between adjacent conductors is not also a good thermal insulator, the helium coolant which flows inward may be warmed by the helium which flows 
outward in the adjacent conductors. Thus, cold helium enters and leaves each pancake but the innermost windings could become overheated.

Figure G-10 shows the location of the $\mathrm{LN}_{2}$ cooled copper shield and tendon collars. By absorbing most of the heat leak from the warm shell at $77 \mathrm{~K}$ instead of at $4 \mathrm{~K}$ in the liquid helium, the refrigeration power required can be reduced to $35 \mathrm{~kW} / \mathrm{coi} 1$.

Figure G-11 shows a detail of one of the lateral support tendons. The main support tendons are of the same configuration but longer $(2.25 \mathrm{~m})$ and larger in diameter $(7.6 \mathrm{~cm})$. The nylon bushing is stressed to $138 \mathrm{MPa}$ (20 ksi) in compression and provides good thermal and electrical insulation.

Figure F-3 shows a plan view of the divertor and two adjacent reactor modules. The field lines are bent through the throat by the reversing and shaping coils. The flux of deuterons, tritons, impurities and helium is then distributed over the face of the divertor plate. The particles become neutral and diffuse through a set of air-cooled louvers and a set of $\mathrm{LN}_{2}$-cooled louvers and are finally collected as solid D-T with intrained He and impurities on a set of liquid helium cooled panels. These panels pivot so that alternate sides of the panels can be exposed to the particle flux while the accumulated D-T on the opposite side evaporates into a duct leading to the fuel reprocessing system. Fig. F-4 shows a cross section of the cryopumping system.

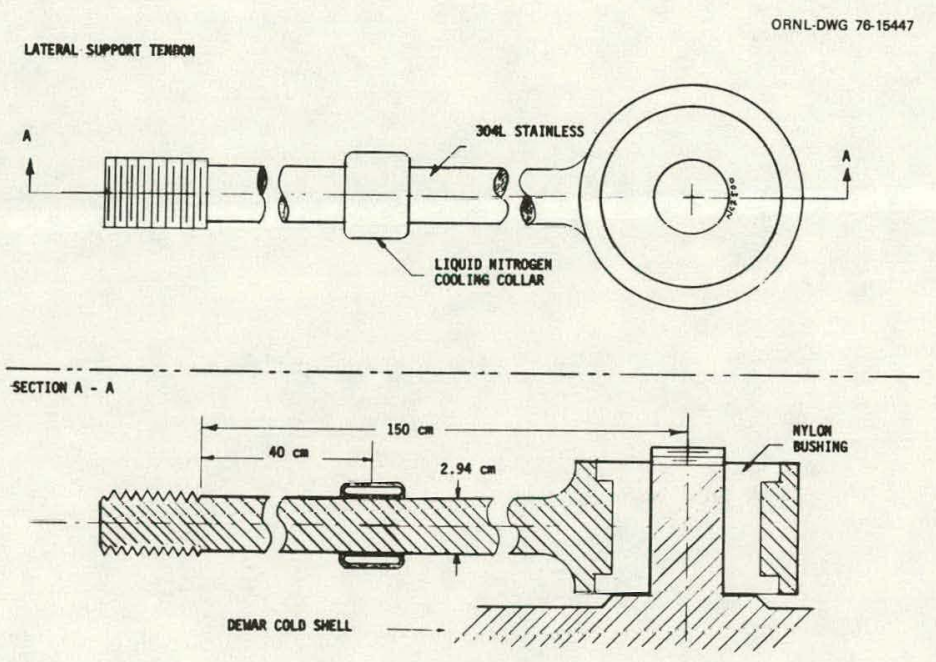

Fig. G-11. Lateral support tendon.

A problem in the impurity control system that is not resolved is the shielding of the divertor coils and the role that charged particles which collide with the lithium cooled surfaces in the divertor throat will play in magnet protection.

The alternate design postulates the use of concrete filled with austenitic iron shot as a shielding material. Neutronics calculations have shown that this shield in the configuration allowed by movable magnets is more effective in reducing the heat deposition by ncutron and ydullid heating 
( $1 \times 10^{-23} \mathrm{~W} / \mathrm{cm}^{3} /$ neutron $/ \mathrm{sec}$ vs $6 \times 10^{-23} \mathrm{~W} / \mathrm{cm}^{3} /$ neutron $/ \mathrm{sec}$ ) than the stainless steel and borated water shield. The primary reason for using concrete, however, is to reduce the amount of stainless steel (and thus chromium and nickel) needed for the reactor. The calculations indicate that 23,000 tons of stainless steel would be needed to adequately shield the EBT. Chromium in particular may be in short supply by the time a fusion economy becomes feasible.

The choice of austenitic iron was made to avoid interference of the shield with the magnetic field. Further research may show that for small enough shot size, ferritic iron shot may be acceptable in this application.

\section{REFERENCE}

1. M. 0. Hoenig, D. B. Montgomery, Y. Iwasa, and J.E.C. Williams, "Forced Supercritical Helium Cooling for Large Toroidal Coils, Phase I Report, "MIT Francis Bitter National Magnet Laboratory, Cambridge. Massachisetts (April 1975). 
APPENDIX H

NEUTRONICS CALCULATIONS

\section{INTRODUCTION}

In this appendix, the results of a preliminary neutronics analysis of the ELMO Bumpy Torus (EBT) reference reactor design are summarized. A discussion of the neutronics investigations proposed as a logical extension of this preliminary study is also given.

The purpose of the initial neutronics calculations was to assess the capability of a lithiumladen prototypic blanket configuration for recovering the kinetic energy of the fusion neutrons and secondary gamma rays in the form of heat, and for breeding tritium. The effectiveness of the shleld surrounding the blanket in reducing the radiation incident on the toroidal magnet coils was also determined by comparing the energy deposition rate and radiation damage in the coils as a function of the shield thickness.

A preliminary neutronics analys is was also carried out for an alternative blanket-shield concept proposed for the EBT. The alternative concept differs from the prototypic blanket-shield design principaliy in the selection of the first wall material, first wall coolant, and in the composition of the shield material.

The preliminary calculations and the results obtained for the prototypic blanket are summarized in Sect. 2; the results obtained for the alternative design are given in Sect. 3 . The calculations that are proposed as part of the continuing research on the EBT reference design are presented in Sect. 4 .

\section{PRELIMINARY NEUTRONICS ANALYSIS}

The preliminary neutronics analyses for the EBT Reactor Reference Design were made using the onedimensional discrete ordinates code ANISN. ${ }^{1}$ The transport calculations were carried out using a $P_{3}$ scattering expansion, an $\mathrm{S}_{12}$ angular quadrature, and coupled 35-group neutron, 21-group gamma-ray cross sections obtained by collapsing the coupled 100-group neutron, 21-group gamma-ray cross section library used for the Experimental Power Reactor (EPR) neutronics studies. ${ }^{2,3}$ Energy deposition was calculated using neutron and gamma-ray kerma factors obtained from the computer codes MACKLIB ${ }^{4}$ and SMUG ${ }^{5}$ respectively. Radiation damage in the structural materials and the magnet coils was estimated using atomic displacement and gas production cross sections generated by the code RECOIL. ${ }^{6}$

The dimensions and materials in the blanket, shield, and magnet coils used in the calculations are summarized in Table H-1. Reactor configurations that include 40-, 50-, and 70-cm-thick shield assemblies were analyzed. For these studies, the magnet coils were assumed to have the same composition as those proposed for the EPR. ${ }^{7}$ Calculations including a graphite liner ${ }^{8}$ located between the plasma and the first wall were used to evaluate the effect of the liner. However, at this time the divertor system described in Appendix $F$ is considered to be the most likely means of impurity control.

The composition of lithium used in the blanket was taken to be that of natural 1 ithium $(7.4 \%$ of ${ }^{6} \mathrm{Li}, 92.6 \%$ of $\left.{ }^{2} \mathrm{Li}\right)$. The main constituents of the shield are borated water $(35 \%)$ and stainless steel type 316 in the form of spheres $(65 \%)$. This composition was selected because it has good radiationattenuating properties and because the heat generated in the shield by the neutrons and secondary gamma rays may be efficiently removed. 
TABLE H-1

Dimensions and Composition of Materials

\begin{tabular}{|c|c|c|c|c|}
\hline \multirow[b]{3}{*}{ Zone } & \multirow[b]{3}{*}{ Material } & \multicolumn{3}{|c|}{ Shield Thickness $(\mathrm{cm})$} \\
\hline & & 40 & 50 & 70 \\
\hline & & & $\begin{array}{l}\text { Outer rad } \\
(\mathrm{cm})\end{array}$ & \\
\hline 1 & Píasma & 100.00 & 100.00 & 100.00 \\
\hline 2 & Cold zone & 120.00 & 120.00 & 120.00 \\
\hline 3 & SS-316 & 120.16 & 120.16 & 120.16 \\
\hline 4 & Salt coolant & 120.80 & 120.80 & 120.80 \\
\hline 5 & $5 S-316$ & 120.96 & 120.96 & 120.96 \\
\hline 6 & Void & 121.60 & 121.60 & 121.60 \\
\hline 7 & $53-310$ & $1 \varepsilon \varepsilon . \varepsilon 4$ & $1 \varepsilon 2.24$ & $1 \varepsilon \varepsilon .24$ \\
\hline 8 & Lithium & 152.24 & 152.24 & 152.24 \\
\hline 9 & SS-316 & 152.88 & 152.88 & 152.88 \\
\hline 10 & Graphite reflector & 162.88 & 162.88 & 162.88 \\
\hline 11 & $S S-316$ & 163.52 & 163.52 & 163.52 \\
\hline 12 & Lithium & 173.52 & 173.52 & 173.52 \\
\hline 13 & SS-316 & 174.16 & 174.16 & 174.16 \\
\hline 14 & Graphite reflector & 179.16 & 179.16 & 179.16 \\
\hline 15 & SS-316 & 179.80 & 179.80 & 179.80 \\
\hline 16 & Lithium coolant & 180.44 & 180.44 & 180.44 \\
\hline 17 & SS-316 & 182.94 & 182.94 & 182.94 \\
\hline 18 & Lithium coolant & 183.58 & 183.58 & 183.58 \\
\hline 19 & SS-316 & 186.08 & 186.08 & 186.08 \\
\hline 20 & Lithium coolant & 186.72 & 186.72 & 186.72 \\
\hline 21 & SS -316 & 189.22 & 189.22 & 189.22 \\
\hline 22 & Void & 209.00 & 209.00 & 209.00 \\
\hline 23 & $S S-316$ & 214.00 & 214.00 & 214.00 \\
\hline 24 & $\mathrm{H}_{2} \mathrm{O} \cdot \mathrm{B}+\mathrm{SS}-316$ & 232.50 & 242.50 & 262.50 \\
\hline 25 & Lead & 244.00 & 254.00 & 274.00 \\
\hline 26 & $\$ S-316$ & 249.00 & 258.00 & 278.00 \\
\hline 27 & Void & 259.00 & 269.00 & 285.00 \\
\hline 28 & \$\$-316 & 269.00 & 279.00 & 299.00 \\
\hline 29 & Magnet coils & 324.00 & 334.00 & 354.00 \\
\hline 30 & $\$ \$-316$ & 334.00 & 344.00 & 364.00 \\
\hline
\end{tabular}

The plasma dimensions and neutron parameters used in the preliminary calculations are summarized in Table $\mathrm{H}-2$. For these calculations, the major radius of the EBT was taken to be $60 \mathrm{~m}$ (EBTR-48). For EBTR-24, some of the results given here must be scaled to account for the difference in the circumferential length of the plasma.

If the EBT is to function as a power-producing reactor, the blanket must be capable of recovering a substantial fraction of the kinetic energy of the fusion neutrons and secondary gamma rays in the form of heat. The shield that surrounds the blanket must serve both as a thermal barrier between the hot blanket and the cryogenic magnet coils and as a radiation-attenuating medium to reduce the magnitude 
of the radiation leaking from the blanket in order to minimize the nuclear heating and radiation damage in the coils. The energy deposition rate as a function of reactor radius for the reactor configuration using the $70-\mathrm{cm}-$ thick shield is shown in Fig. $\mathrm{H}-1$.

TABLE H-2

\section{EBTR Reference Design Parameters}

$\begin{array}{lc}\text { Major radius }(\mathrm{m}) & 60.0 \\ \text { Minor radius }(\mathrm{m}) & 1.2 \\ & 1.71 \times 10^{9} \\ \text { Plasma volume }\left(\mathrm{cm}^{3}\right) & 2.84 \times 10^{7} \\ \text { Plasma area }\left(\mathrm{cm}^{2}\right) & 3.77 \times 10^{4} \\ \text { Plasma length }(\mathrm{cm}) & 1.0 \\ & 4.43 \times 10^{13} \\ \text { Neutron wall loading }\left(\mathrm{MW} / \mathrm{m}^{2}\right) & 3.34 \times 10^{16} \\ \text { Neutron flux }\left(\mathrm{n} / \mathrm{cm}^{2} / \mathrm{sec}\right) & \end{array}$

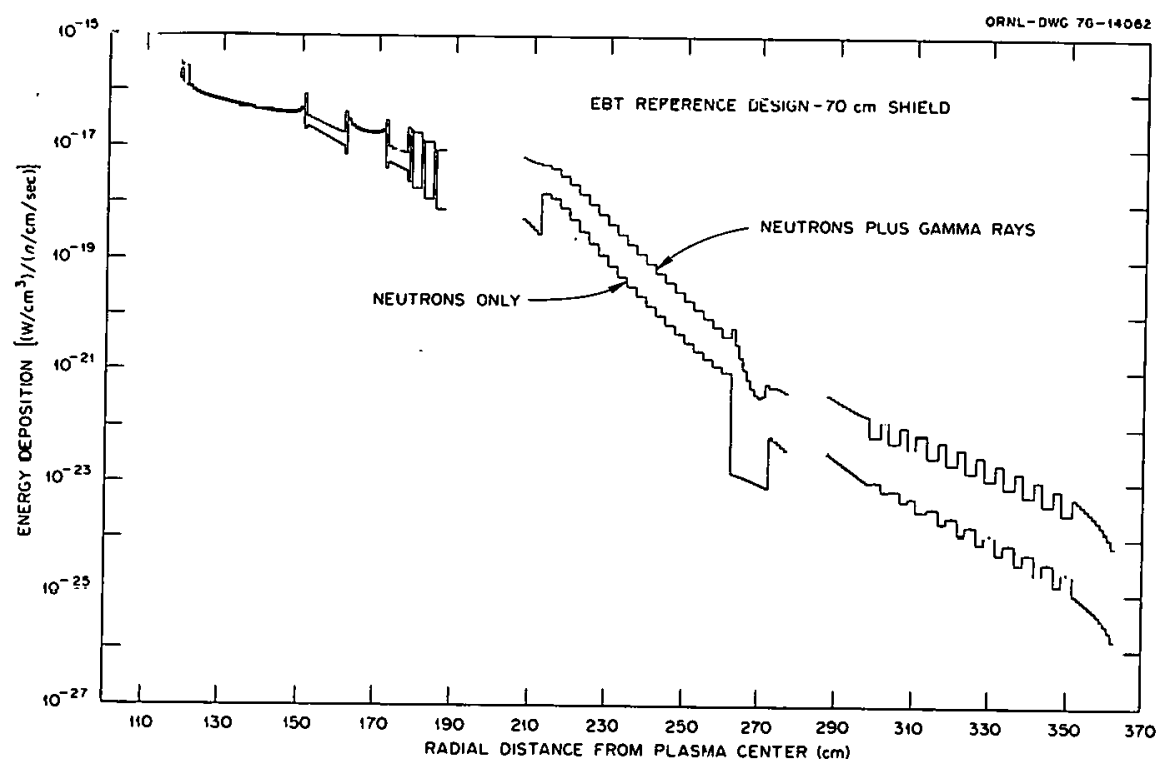

Fig. H-1. Energy deposition rate as a function of reactor radius.

The upper curves show the energy deposition rate by neutrons and secondary gamma rays and the lower curves show the energy deposition rate from neutrons only. More than $90 \%$ of the energy deposited in the magnet coils is due to secondary gamma rays. The fractions of the total energy deposition in the blanket, shield, and magnet coils as a function of the three-shield thicknesses treated in this st.undy 
are given in Table $\mathrm{H}-3$. In all three configurations, approximately $96 \%$ of the energy of fusion is recovered in the blanket. The significant point to note is the reduction in the fractional energy deposition in the magnet assembly with increasing shield thickness.

TABLE H-3

Energy Deposition (in percent) in the EBTR Reference Design

\begin{tabular}{|c|c|c|c|}
\hline & \multicolumn{3}{|c|}{$\begin{array}{c}\text { Shield Thickness } \\
(\mathrm{cm})\end{array}$} \\
\hline & 40 & 50 & 70 \\
\hline Blanket & 96.263 & 96.266 & 96.250 \\
\hline Shield & 3.72 & 3.73 & 3.74 \\
\hline Magnet assembly ${ }^{a}$ & 0.017 & 0.004 & 0.0002 \\
\hline
\end{tabular}

dThe magnet assembly includes the structural supports and the magnet coil.

The energy deposition rate in the magnet coil, $P_{C}$, may be inferred approximately by the relation

$$
P_{c}=\left(P_{n} \times f \times A\right) / N_{c}
$$

where

$P_{n}=$ total neutron-produced power in the reactor,

$f=$ the fraction of the energy deposited in the magnet coils (from Table $\mathrm{H}-3$ ),

$A=$ the ratio of the surface area of the magnet coils to the total reactor surface area at the inner radius of the coil faces, and

$\mathrm{N}_{\mathrm{c}}=$ the number of magnet coils.

Then for a shield thickness of $70 \mathrm{~cm}$ and an assumed value of 0.5 for $A$, the energy depusition rate in the 48-coil magnet configuration is $2 \times 10^{-8} P_{n} W$ per magnet-coil assembly. This value is based on the results of a one-dimensional calculation and does not take into account any heatiny due lu radiation that may stream through injector, vacuum, or diagnostic penetrations in the blankel dild shield. The effects of radiation streaming must be evaluated using multidimensional radiation transport methods, so the result given above must be taken to be conservative.

The fusion neutrons interact with the lithrum in the blanket and produce lriliunl via tlie reactiluis

$$
6_{L i}(n, \alpha) T+4.78 \mathrm{MeV}
$$

and

$$
\text { 'Li }\left(n, n^{\prime} \alpha\right) T-2.47 \mathrm{MeV} .
$$

The tritium breeding ratios (tritium nuclei per incident neutron) in the blanket are given in Table $H-4$. The tritium breeding was also obtained from a one-dimensional calculation, so the value of 1.35 must be considered to be optimistic since no account is made for the penetrations in the blanket. The value for the breeding is sufficiently large, however, that even when the penetrations are considered, a fertile blanket will be maintained. 
TABLE H-4

Tritium Breeding Ratios in the EBT Blanket

\begin{tabular}{|c|c|c|c|c|}
\hline \multirow[b]{2}{*}{ Zone } & \multirow[b]{2}{*}{$\begin{array}{l}\text { Thickness } \\
(\mathrm{cm})\end{array}$} & \multicolumn{3}{|c|}{ Tritium Nuclei/Incident Neutron } \\
\hline & & ${ }^{6}{ }_{\mathrm{Li}}$ & ${ }^{7}{ }_{L i}$ & ${ }^{6} \mathrm{Li}+{ }^{7} \mathrm{Li}$ \\
\hline 8 & 30 & 0.627 & 0.472 & 1.099 \\
\hline 12 & 10 & 0.196 & 0.024 & 0.220 \\
\hline 16 & 0.64 & 0.015 & 0.001 & 0.016 \\
\hline 18 & 0.64 & 0.010 & 0.0004 & 0.0014 \\
\hline 20 & 0.64 & 0.007 & 0.0002 & 0.0072 \\
\hline & Totals: & 0.855 & 0.498 & 1.353 \\
\hline
\end{tabular}

The neutron-induced radiation damage in the first structural wall and in the magnet-coil windings will determine the maintenance and/or replacement interval for these components. The atomic displacement rates and gas production rates in the SS-316 first structural wall for a neutron wall loading of $1 \mathrm{MW} / \mathrm{m}^{2}$ are given in Table $\mathrm{H}-5$. These values are consistent with those obtained by other authors for similar materials at the same wall loading. 9.10 The atomic displacement rates and gas production rates in the first winding of the magnet coil as a function of shield thickness are summarized in Tables $\mathrm{H}-6$ and $\mathrm{H}-7$ respectively.

TABLE H-5

Radiation Damage in the EBT First Structural Wall

Composition $=$ SS-316, Neutron Wal1 Loading $=1.0 \mathrm{MW} / \mathrm{m}^{2}$ )

Atomic displacement rate (dpa/year)

10.71

Gas production rates (appm/year):

$\begin{array}{lr}\text { hydrogen } & 444.38 \\ \text { helium } & 131.21 \\ \text { deuterium } & 3.06\end{array}$

TABLE $\mathrm{H}-6$

Radiation Damage in the EBT Coil - First Winding (Neutron Wal1 Loading $=1.0 \mathrm{MW} / \mathrm{m}^{2}$ )

Atomic Displacement Rates (dpa/year) ${ }^{\mathrm{a}}$

Shield Thickness (cm)

Material

\begin{tabular}{cccc}
\hline 40 & $\frac{50}{20}$ & & 70 \\
$3.14 \times 10^{-4}$ & & $6.95 \times 10^{-5}$ & $3.81 \times 10^{-6}$ \\
$4.21 \times 10^{-4}$ & $9.32 \times 10^{-5}$ & $5.11 \times 10^{-6}$ \\
$4.44 \times 10^{-4}$ & $9.79 \times 10^{-5}$ & $5.37 \times 10^{-6}$ \\
$7.58 \times 10^{-4}$ & & $1.66 \times 10^{-4}$ & $9.11 \times 10^{-6}$
\end{tabular}

SS-316

$7.58 \times 10^{-4}$

$1.66 \times 10^{-4}$

$9.11 \times 10^{-6}$

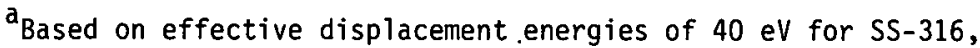
$60 \mathrm{eV}$ for $\mathrm{Nb}, 30 \mathrm{eV}$ for $\mathrm{Cu}$, and $25 \mathrm{eV}$ for $\mathrm{Al}$. 
Values are given for $\mathrm{SS}-316, \mathrm{Nb}, \mathrm{Cu}$, and $\mathrm{Al}$, all of which are contained in, or in the immediate vicinity of, the coil winding. The values are small and, for the proposed neutron wall loading, do not appear to have any significant influence on the performance of the magnets.

TABLE $\mathrm{H}-7$

Radiation Damage in the EBT Coil - First Winding (Neutron Wal1 Loading $=1.0 \mathrm{MW} / \mathrm{m}^{2}$ )

Gas Production Rates (appm/year)

Shield Thickness $(\mathrm{cm})$

\begin{tabular}{|c|c|c|c|}
\hline Material & 40 & 50 & 70 \\
\hline \multicolumn{4}{|l|}{ SS-316 } \\
\hline $\mathrm{H}$ & $8.92 \times 10^{-4}$ & $3.20 \times 10^{-4}$ & $1.30 \times 10^{-5}$ \\
\hline $\mathrm{He}$ & $\dot{2} .07 \times 10^{-4}$ & $5.10 \times 10^{-5}$ & $3.00 \times 10^{-6}$ \\
\hline
\end{tabular}

$\underline{\mathrm{Nb}}$

$\begin{array}{llll}H & 1.63 \times 10^{-4} & 4.01 \times 10^{-5} & 2.36 \times 10^{-6} \\ \mathrm{He} & 3.53 \times 10^{-5} & 8.70 \times 10^{-6} & 5.09 \times 10^{-7}\end{array}$

Cu

H $\quad 1.02 \times 10^{-3} \quad 2.50 \times 10^{-4} \quad 1.46 \times 10^{-5}$

$\mathrm{He}$

$1.34 \times 10^{-4}$

$3.32 \times 10^{-5}$

$2.41 \times 10^{-6}$

$\underline{\text { Al }}$

$\begin{array}{llll}H & 4.38 \times 10^{-4} & 1.09 \times 10^{-4} & 0.43 \times 10^{-6} \\ \text { He } & 4.36 \times 10^{1} & 1.08 \times 10^{-1} & 6.34 \times 10^{-6}\end{array}$

On the basis of these preliminary one-dimensional calculations, the following observations and conclusions are noted:

1. The energy deposition rate in the blanket is high enough to achieve useful extraction of heat for the generation of power.

2. A 70-cm-thick shield should be used if there is sufficient space between the blanket and the coils since (a) the heating rates in the magnet can be tolerated and the use of thinner shields would result in unreasonably high coil heating, and (b) the radiation damage in the coils is smaīi.

3. The high tritium breeding ratio (1.35) provides enough latitude to allow the inclusion of required ports and penetrations and still achieve a fertile reactor.

These calculations do not take into account the effecls of perietrations, and until more detinitive multidimensional calculations that include these effects are made, these results should be considered optimistic.

3. ANALYSIS OF ALTERNATIVE BLANKET-SHIELD DESIGNS

The alternative blanket-shield designs that have been proposed for use in the EBT are given in Table H-8. These designs differ from those given in Table H-1 principally by: 
1. the use of niobium as the first wall material,

2. the substitution of "heavy" concrete ( $40 \% \mathrm{Fe}, 60 \%$ ordinary concrete) for borated water plus stainless steel as the shield material, and

3. the elimination of the maintenance void separating the blanket and the shield. The combined thickness of the blanket and shield is $165 \mathrm{~cm}$ and the toroidal magnet coil assembly is separated from the shield by a $14-\mathrm{cm}$-thick void region. The magnet coil assembly materials and dimensions used in these calculations was taken to have the same dimensions and composition as that given in Table $\mathrm{H}-1$.

TABLE $\mathrm{H}-8$

Alternative Blanket-Shield Designs

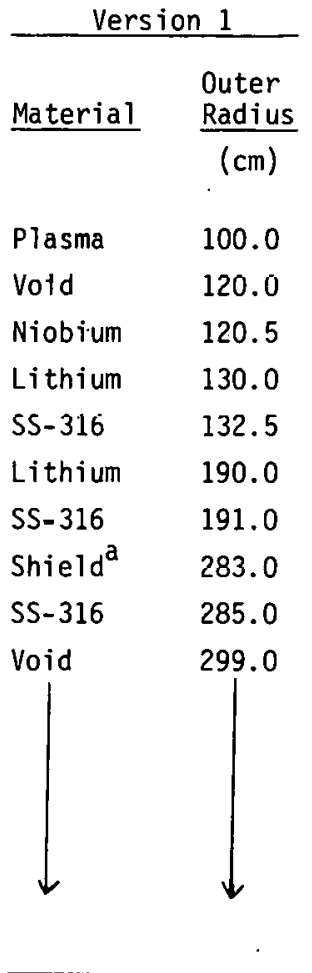

\begin{tabular}{l} 
Ver \\
Materia \\
\hline Plasma \\
Void \\
Niobium \\
Lithium \\
Carbon \\
SS-316 \\
Shie1d \\
SS-316 \\
Lead \\
SS-316 \\
Void \\
$\downarrow$
\end{tabular}

Version 2

\section{Outer \\ Radius}

(cm)

100.0

120.0

120.5

175.0

190.0

191.0

277.0

278.0

283.0

285.0

299.0

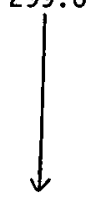

Magnet Coil Assembly
Version 3

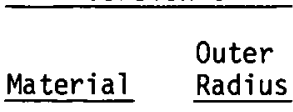

(cm)

$\begin{array}{ll}\text { Plasma } & 100.0 \\ \text { Void } & 120.0 \\ \text { Niobium } & 120.5 \\ \text { Lithium } & 140.5 \\ \text { SS-316 } & 141.5 \\ \text { Carbon } & 151.5 \\ \text { SS-316 } & 152.5 \\ \text { Lithium } & 190.0 \\ \text { SS-316 } & 191.0 \\ \text { Shield } & 277.0 \\ \text { SS-316 } & 278.0 \\ \text { Lead } & 283.0 \\ \text { SS-316 } & 285.0 \\ \text { Void } & 299.0 \\ \downarrow & \downarrow\end{array}$

Version 4

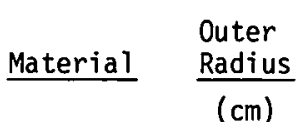

$\begin{array}{ll}\text { Plasma } & 100.0 \\ \text { Void } & 120.0 \\ \text { Niobium } & 120.5 \\ \text { Lithium } & 130.5 \\ \text { SS-316 } & 131.5 \\ \text { Carbon } & 141.5 \\ \text { SS-316 } & 142.5 \\ \text { Lithium } & 190.0 \\ \text { SS-316 } & 191.0 \\ \text { Shield } & 283.0 \\ \text { SS-316 } & 285.0 \\ \text { Void } & 299.0 \\ \downarrow & \downarrow\end{array}$

${ }^{\mathrm{a}}$ Shieìd is composed of $40 \% \mathrm{Fe} \cdot 60 \%$ concrete.

The tritium breeding ratios (tritium nuclei per incident neutron) in the blanket in each of the candidate alternative designs are summarized in Table $\mathrm{H}-9$. Each of the designs yields a fertile system with "Version 2" being the most effective breeder, both in terms of the breeding ratio and in terms of the tritium yield per cubic centimeter of lithium. In all of the designs, the tritium breeding is enhanced by the presence of the niobium first wall; this is mainly due to the large ( $n, 2 n$ ) cross section ( $\sim 1300 \mathrm{mb}$ ) in niobium.

The energy deposition rate in the first winding of the magnet coil is compared in Table $\mathrm{H}-10$ as a function of the blanket-shield configurations. Version 2 represents the most effective system in reducing the heating rate in the magnet. 
TABLE H-9

Tritium Breeding Ratios

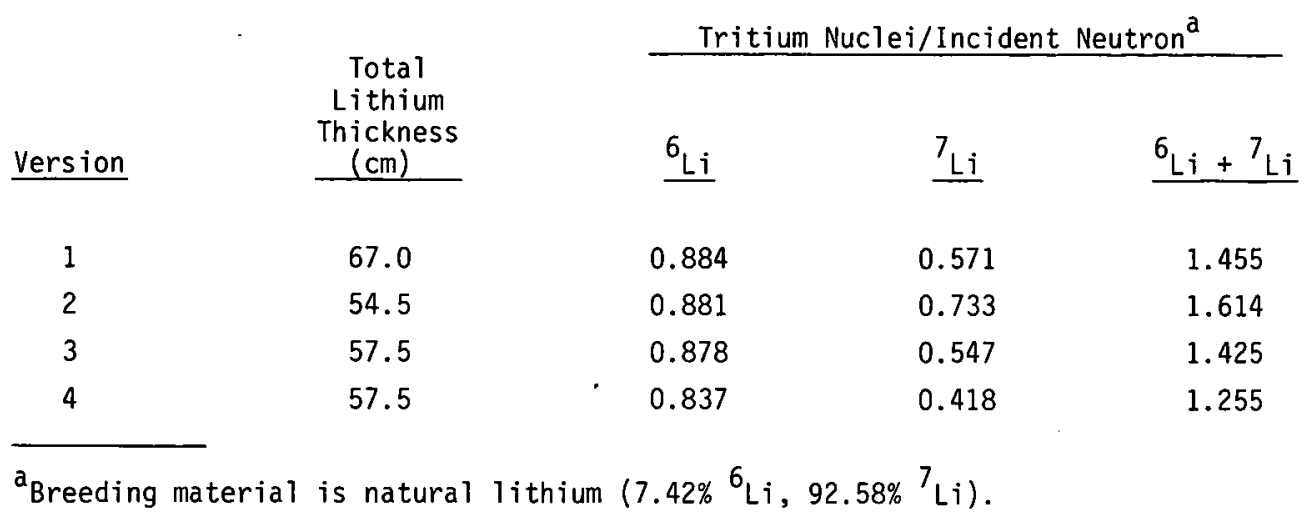

TABLE H-10

Energy Deposition Rates in the First Winding of the Toroidal Magnet Coil $\left(\mathrm{W} / \mathrm{cm}^{3}\right) /(\mathrm{n} / \mathrm{s} / \mathrm{cm})$

$\begin{array}{cccc}\text { Version } & \text { Neutron } & \text { Gamma Ray } & \text { Total } \\ 1 & 4.99 \times 10^{-25} & 9.85 \times 10^{-24} & 1.03 \times 10^{-23} \\ 2 & 2.96 \times 10^{-25} & 2.52 \times 10^{-24} & 2.78 \times 10^{-24} \\ 3 & 6.11 \times 10^{-25} & 7.54 \times 10^{-24} & 8.15 \times 10^{-24} \\ 4 & 3.55 \times 10^{-25} & 7.11 \times 10^{-24} & 7.47 \times 10^{-24}\end{array}$

4. FUTURE NEUTRONICS CALCULATIONS

In the continuing study of the EBT reference design, one-dimensional neutronics calculations should be used to estimate the induced activity, afterheat, and biological shielding requirements. The activation and afterheat data are necessary for planning the remote maintenance procedures and the requirements for storing and cooling reactor components being repaired or salvaged. The determination of biological shielding requirements provides a basis for building and containment structures that assure the proper radiation protection of personnel and equipment located in the vicinity of the reactor.

The main effort in the neutronics analysis of the EBT components should be accomplished using multidimensional radiation transport methods. The geometry of the reactor, i.e., the large aspect ratio, lends itself to two-dimensional neutronics analysis. The integrity of the magnet shielding in isolating the coils from the plasma radiation may be determined in terms of the spatial distributions of the nuclear heating and radiation damage in the coils. Data of this type will assist the design engineers in determining the optimum shielding configuration in a cost-effective manner.

The effects on reactor and component performance and lifetimes due to penetrations in the blanket and shield that are required for vacuum pumping, diagnostic instrumentation, and neutral beam injection are best evaluated using three-dimensional Monte Carlo methods. The influence of these ports on lithium breeding in the blanket and radiation damage and nuclear heating in vital components of the 
reactor must be estimated. The large openings required for injection and vacuum pumping may also lead to excessive radiation damage to vital parts in the injector or pump, shortening their operating lifetimes.

The neutronics commitment must be consistent with the design progress on the EBT and provide relevant information for evaluating the reactor and component designs. This effort must parallel the total research effort and be carried out in a coordinated manner with all other research areas.

\section{REFERENCES}

1. W. W. Engle, Jr. A User's Manual for ANISN, a One-Dimensional Discrete Ordinates Transport Code with Anisotropic Scattering, Report K-1693, Computing Technology Center, Union Carbide Corporation, Oak Ridge (1967).

2. D. M. Plaster, R. T. Santoro, and W. E. Ford III, Coupled 100-Group Neutron and 21-Group Gamma-Ray Cross Sections for EPR Calculations, ORNL/TM-4872, Oak Ridge (1975).

3. W. E. Ford III, R. T. Santoro, R. W. Roussin, and D. M. Plaster, Modification Number One to the Coupled 100n-21Y Cross Section Library for EPR Calculations, ORNL/TM-5249, Oak Ridge (1976).

4. M. A. Abdou and R. W. Roussin, MACKLIB - 100-Group Neutron Fluence-to-Kerma Factors and Reaction Cross Sections Generated by the MACK Computer Program from Data in ENDF Format, 0RNL/TM-3995, 0ak Ridge (1974).

5. N. M. Greene et al., AMPX: A Modular Code System for Generating Coupled Multigroup Neutron-Gamma Libraries from ENDF/B, ORNL/TM-3706, Oak Ridge (1976).

6. T. A. Gabriel, J. D. Amburgey, and N. M. Greene, Radiation-Damage Calculations: Primary Recoil Spectra, Displacement Rates, and Gas-Production Rates, ORNL/TM-5160, 0ak Ridge (1976).

7. M. Roberts and E. S. Bettis, Oak Ridge Tokamak Experimental Power Reactor Study - Reference Design, ORNL/TM-5042, Oak Ridge (1975).

8. G. L. Kulcinski et al., A Method to Reduce the Effects of Plasma Contamination and First Wall Erosion in Fusion Reactors, UWFDM-108, University of Wisconsin, Madison, Wisconsin (1974).

9. G. L. Kulcinski, D. G. Doran, and M. A. Abdou, "Comparison of the Displacement and Gas Production Rates in Current Fission and Future Fusion Reactors," University of Wisconsin, 1974 (unpublished).

10. M. L. Williams, R. T. Santoro, and T. A. Gabriel, The Performance of Various Structural Materials in Fusion-Reactor Blankets, ORNL/TM-5036, 0ak Ridge (1976); to be published in NucT. Tech. 
1-3. Lab Records

4. Lab Records - RC

5. $Y-12$ Document Reference

6-7. Central Research Library

8-9. Fusion Energy Library

10. ORNL Patent Office

11. L. A. Berry

12. E. S. Bettis

13. P. B. Burn

14. J. D. Callen

15. J. F. Clarke

16-25. R. A. Dand 1

26. R. A. Dory

27. G. R. Haste

28-32. C. L. Hedrick

33. H. Ikegami

34. E. F. Jaeger

35. N. H. Lazar

36. G. G. Kelley

37-71. D. G. McAlees

72. 0. B. Morgan

73. D. B. Nelson

74. L. W. Owen

75. H. Postma

76. J. F. Roberts

77. M. N. Rosenthal

78. R. T. Santoro

79. D. A. Spong

80. D. Steiner

81-123. N. A. Uekan

124. H. T. Yeh

125. H. L. Watts

126-165. Prof. L. M. Lidsky, Massachusetts Institute of Technology, Department of Nuclear Engineering, Cambridge, MA 02139

166-170. - Dr. D. A. Ehst, ANL-CTR Program, B1dg. 208, Argonne National Laboratory, Argonne, IL 60439

171-175. Dr. A. Pant, Department of Nuclear Engineering, Massachusetts Institute of Technology, Cambridge, MA 02139

176. Dr. C. C. Baker, General Atomic Company, P. 0. Box 81608, San Diego, CA 92138

177. Dr. 1. P. Boris, Plasma Physics, Naval Research Laboratory, Washington, DC 20390

178. Prof. F. Chen, Department of Physics, University of California, Los Angeles, CA 90024

179. Dr. M. Clark, Jr., Combustion Eng., C-E Fast Breeder Development, Windsor, CT 06095

180. Dr. F. E. Coffman, Division of Magnetic Fusion Energy, Energy Research and Development Administration, Washington, DC 20545

181. Prof. R. W. Conn, Nuclear Engineering Department, University of Wisconsin, Madison, WI

182. Dr. J. G. Cordey, Culham Laboratory, UKAEA, Abingdon, Oxon, UX14, 3DB, United Kingdom

183. Prof. J. N. Davidson, School of Nuclear Engineering, Georgia Tech., Atlanta, GA 30332

184. Dr. N. A. Davies, Division of Magnetic Fusion Energy, Energy Research and Development Adininistration, Washington, OC 20545

185. Dr. S. 0. Dean, Division of Magnetic Fusion Energy, Fnergy Research and Development Administration, Washington, DC 20545

186. Dr. W. Ellis, Division of Magnetic Fusion Energy, Energy Research and Development Administration, Washington, DC 20545

187. Dr. C. A. Flanagan, Fusion Power Systems Department, Westinghouse, P. 0. Box 10864, Pittsburgh, PA 15236

188. Bibliothéque, Service du Confinement des Plasmas, C.E.A., B.P. No. 6, 92, Fontenay-aux-Roses (Seine) France

189. Dr. H. K. Forsen, Laser Enrichment Department, Exxon Nuclear Co., Inc. Bellevue, WA 98009

190. Dr. T. K. Fowler, University of California, Lawrence Rádiation Lab., Livermore, CA 94551

191. Prof. B. D. Fried, Physics Department, University of California, Los Angeles, CA 90024

192. Dr. H. P. Furth, Princeton Plasma Physics Laboratory, Princeton University, P. 0. Box 451 Princeton, NJ 08540

193. Dr. G. Gibson, Westinghouse Electric Corporation, Fusion Power Systems, Cheswick, PA 15024

194. Dr. M. B. Gottlieb, Plasma Physics Laboratory, Princeton University, P. 0. Bnx 451, Princeton, NJ 08540 
195. Dr. W. C. Gough, Electric Power Research Institute, 3412 Hillview Avenue, Palo Alto, CA

197. Dr. H. Grad, Courant Institute of Math. Sci., New York Universily, New York, NY 10012

198. Prof. R. Gross, Plasma Research Laboratory, Columbia University, New York, NY 10027

199. Dr. G. E. Guest, General Atomic Company, P. 0. Box 81608, San Diego, CA 92138

200. Dr. R. Harder, General Atomic Company, P. 0. Box 81608, San Diego, CA 92138

201. Dr. R. Hancox, Culham Laboratory, UKAEA, Abingdon, Oxon, 0x14, 3DB, United Kingdom

202. Dr. A. F. Haught, United Technologies Research Labs, East Hartford, CT 06108

203. Dr. C. Henning, Division of Magnetic Fusion Energy, Energy Research and Development Administration, Washington, DC 20545

204. Dr. R. L. Hirsch, Division of Magnetic Fusion Energy, Energy Research and Development Administration, Washington, DC 20545

205. Or. V. I. Ivanov, Physical-Technical Institute of the Ukranian Academy of Sciences, Sukhumi, USSR

206. Prof. T. Kammash, Department of Nuclear Engineering, University of Michigan, Ann Arbor, MI 48109

207. Dr. E. E. Kintner, Division of Magnetic Fusion Energy, Energy Research and Development Administration, Washington, DC 20545

208. Dr. R. N. Kostoff, Division of Magnetic Fusion Energy, Energy Research and Development Administration, Washington, DC 20545

209. Dr. N. A. Kral1, Science Applicatioris, Inc., P. 0. Box 2354, La Jolla, CA 92037

210. Prof. G. L. Kulcinski, Nuclear tngineerlny Departirent, University of Wisconsin, Madicon, WI

211. Ruth Lengye, Bibliothek, Max Planck Institut für Plasmaphysik, 8046 Garching bei München, Federal Republic of Germany

21. I ihrary, International Centre for Theoretical Physics, Trieste, Italy

213. Library, FOM-Institut voor Hlasma-Fys 1Ca, RIJuhusell, Jutilias, Netherlands

214. Librarian, Culham Laboratory, UKAEA, Abingdon, Oxan, OX14, 30B, United Kingdom

215. Library, Centre de Recherches en Physique des Plasma, 21 Avenue des Bains, 1007, Lausanne, Switzerland

216. Dr. Uscar Manley, Division of Magnetic Fusion Energy, Energy Research and Development Adrministration, Washington, DC 20545

217. Prof. G. H. Miley, Department of Nuclear Engineering, University of Illinois, Urbana, IL 61801

218. Dr. R. G. Mills, Plasma Physics Laboratory, Princeton University, P. 0. Box 451, Princeton, NJ 08540

219. Dr. B. Miller, Division of Magnetic Fusion Energy, Energy Research and Development Administration, Washington, DC 20545

220. Dr. K. G. Moses, Division of Magnetic Fusion Energy, Energy Research and Development Administration, Washington, DC 20545

221. Dr. M. Murphy, Division of Magnetic Fusion Energy, Energy Research and Development Administration, Washington, OC 20545

222. Dr. J. 0. Neff, Division of Magnetic Fusion Energy, Energy Research and Development Administration, Washington, DC 20545

223. Dr. T. Okhawa, General Atomic Company, P. 0. Box 81608, San Diego, CA 92138

224. Dr. E. Oktay, Division of Magnetic Fusion Energy, Energy Research and Development Administration, Washington, DC 20545

225. Plasma Physics Group, Department of Engineering Hhysics, Australian Natiundi University, P. 0. Box 4, Canberra A.C.T., 2600 Australia

226. Dr. J. Powe11, Brookhaven National Laboratory, Upton, Long Is Iand 11973

227. Dr. R. E. Price, Division of Magnetic Fusion Energy, Energy Research and Development Administration, Washington, DC 20545

228. Dr. F. L. Ribe, Los Alamos Scientific Laboratory, P. 0. Box 1663, Los Alamos, NM 87544

229. Dr. M. S. Rabinovich, Lebedev Institute of Physics, Academy of Sciences of the USSR, Leninsky Prospect 53, Moscow, USSR

230. Prof. D. J. Rose, Massachusetts Institute of Technology, Department of Nuclear Engineering, Cambridge, MA 02139

231. Prof. M. N. Rosenbluth, Institute for Advanced Study, Princeton, NJ 08540

232. Dr. D. W. Ross, Physics Department, University of Texas, Austin, TX 78712

2.33. Dr. D. Pfirsch, Institute for Plasma Physics, 8046 Garching bei München, Federal Republic of Germany

234. Dr. W. Sadowski, Division of Magnetic Fusion Energy, Energy Research and Development Administration, Washington, DC 20545

235. Dr. Z. M. Shapiro, Westinghouse Electric Corporation, Fusion Power Systems, Cheswick, PA 15024

236. Dr. A. Sleeper, Division of Magnetic Fusion Energy, Energy Research and Development Administration, Washington, DC 20545

237. Or. W. M. Stacey, Jr., ANL-CTR Program, BIdg. 208, Argonne National Laboratory, Argonne, IL 60439

238. Dr. K. I. Thomassen, Los Alamos Scientific Laboratory, P. 0. Box 1663, Los Alamos, NM 87544

239. Prof. A. W. Trivelpiece, Maxwell Laboratories, Inc., 9244 Balboa Avenue, San Diego, CA 92123

240. Thermonuclear Laboratory, Kurchatov Institute of Atomic Energy, P. 0. Box 3402, Moscow, USSR

241. Thermonuclear Library, Japan Atomic Energy Research Institute, Tokai, Naka, Ibaraki, Japan 
242. Dr. T. C. Varljen, Westinghouse Electric Corporation, Fusion Power Systems, Cheswick, PA

243. Dr. J. M. William, Division of Magnetic Fusion Energy, Energy Research and Development Administration, Washington, DC 20545

244. A. M. Dupas, Documentation S.I.G.N., Department de La Physique du Plasma, Et de La Fusion Controlee, Association EURATOM-CEA, Sur La Fusion, Centre d'Etudes Nucleaires, BP 85 Centre Du TRI 38041 Grenoble Cedex, France

245. Dr. H. H. Woodson, Department of Electrical Engineering, University of Texas, Austin, TX 78712

246. CTR Reading Room, c/O Dr. R. N. Sudan, Phillips Hall, Cornell University, Ithaca, NY 18450

247. Plasma Physics Library, Plasma Physics Laboratory, Princeton University, Forrestal Campus, P. 0. Box 451, Princeton, NJ 08540

248. Controlled Thermonuclear Research Library, Lawrence Livermore Laboratory, P. 0. Box 808 Livermore, CA 94550

249. CTR Library, General Atomic Company, P. O. Box 81608, San Diego, CA 92138

250. CTR Reading Room, c/o Prof. A. N. Kaufman, Physics Department, University of California, Berkeley, CA 94720

251. Director, Research and Technical Support Division, Eneray Research and Development Administration, Oak Ridge Operations, P. C. Lox E, nak Ridge, TN 37830

252-278. Technical Information Center, Energy Research and Development Administration, P. 0. Box 62, Oak Ridge, TN 37830 